\title{
ELASTIC PROPERTIES OF CEMENT PHASES USING \\ MOLECULAR DYNAMIC SIMULATION
}

\author{
by \\ Mohamed Arar \\ B. Eng., INFORBA Institute, Algiers, Algeria, 1991
}

\author{
A Thesis \\ Presented to Ryerson University \\ In Partial Fulfillment of the \\ Requirements for the degree of \\ Master of Applied Science \\ in the Program of \\ Civil Engineering
}

Toronto, Ontario, Canada, 2016

(C) Mohamed Arar 2016 


\section{AUTHOR'S DECLARATION}

I hereby declare that I am the sole author of this thesis. This is a true copy of the thesis, including any required final revisions, as accepted by my examiners.

I authorize Ryerson University to lend this thesis to other institutions or individuals for the purpose of scholarly research

I further authorize Ryerson University to reproduce this thesis by photocopying or by other means, in total or in part, at the request of other institutions or individuals for the purpose of scholarly research.

I understand that my thesis may be made electronically available to the public. 


\title{
ELASTIC PROPERTIES OF CEMENT PHASES USING MOLECULAR DYNAMIC SIMULATION
}

Mohamed Arar, Master of Applied Science, Civil Engineering, Ryerson University,2016

\begin{abstract}
The goal of this thesis is to shed light on the elastic properties, especially the Young modulus, of clinker phases and hydration products of cement paste through molecular dynamic simulation by COMPASS force field. The parameters that can affect the elastic properties of cement phases were also targeted, with special attention paid to analog C-S-H minerals, in which the Tobermorite family and Jennite were simulated to render their structures representative of C-S-H structure.

The molecular dynamic simulations of this study show that $\mathrm{CO}$ force field can be applicable for most clinker phases and hydration products. Jennite, with its porosity and finite silicate chain accounted for, can be considered the closer and representative structure of C-S-H. In addition, this study confirms the important effect of $\mathrm{C} / \mathrm{S}$ ratio, packing factor and chain length on elastic properties of C-S-H.
\end{abstract}




\section{Acknowledgements}

In the name of Allah, the most gracious and the most merciful. All praises to Allah for the strengths and his blessing in completing this thesis. I would like to express my sincere gratitude to my research supervisor Dr. Mohamed Lachemi for his support, guidance, patience, and trust towards the successful completion of this thesis. Without his support, I would have been unable to accomplish this study.

I would also like to thank Dr. Hocine Siad for his full support and help during the review and completion of this thesis.

I also extend my special thanks to examining committee members: Dr. Medhat Shehata and Dr. Khandaker M. Anwar Hossain 


\section{Dedication}

This thesis is dedicated to the memory of my father. It is also dedicated to my mother, my wife, my wonderful son and two daughters, and to all my family who supported and helped me accomplish my goal in attaining higher education. 


\section{Contents}

AUTHOR'S DECLARATION..............................................................................................................

ABSTRACT ............................................................................................................................................... III

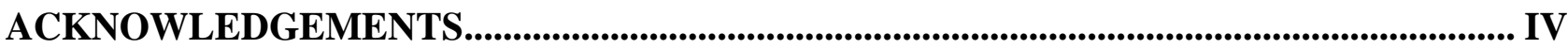

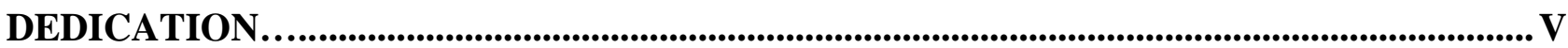

LIST OF TABLES ......................................................................................................................................... XI

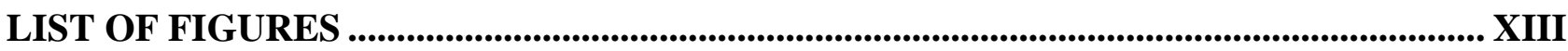

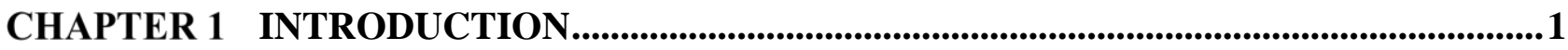

CHAPTER 2 CHEMISTRY OF PORTLAND CEMENT .............................................................6

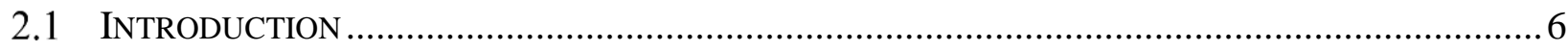

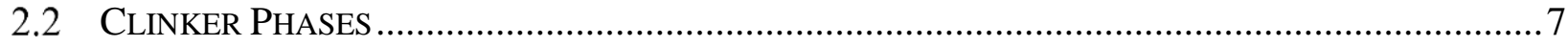

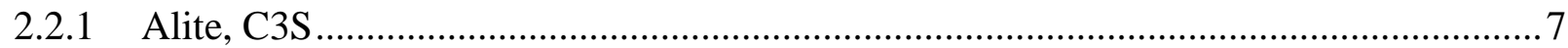

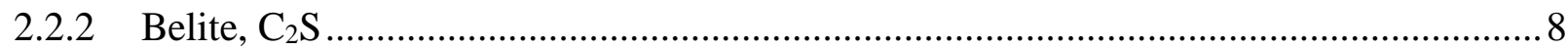

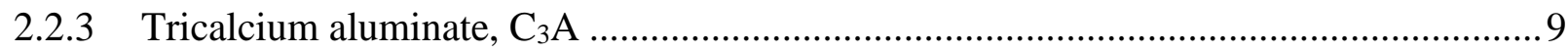

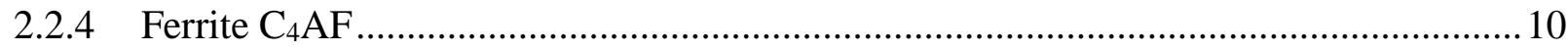

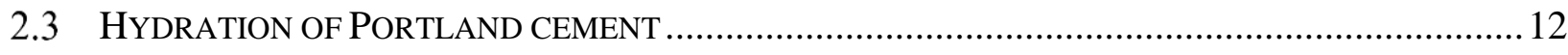

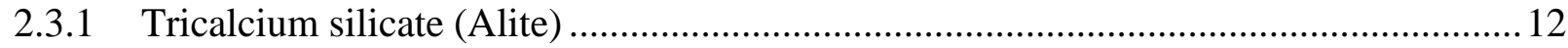

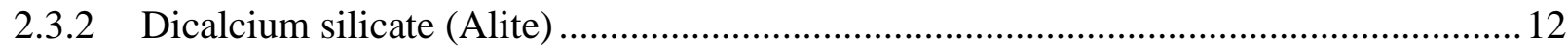

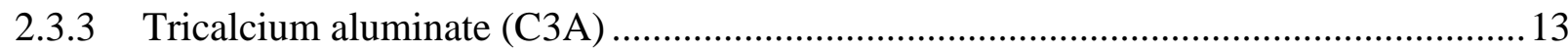

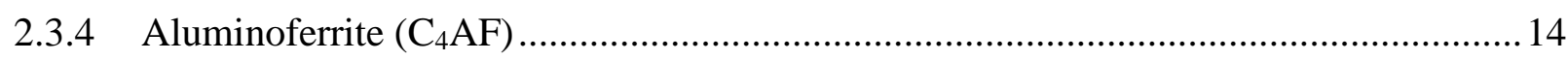

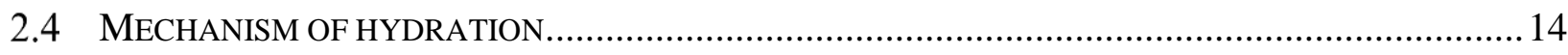

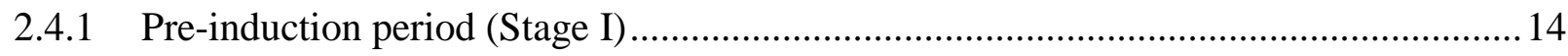

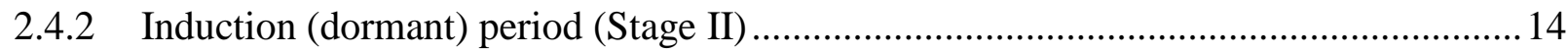

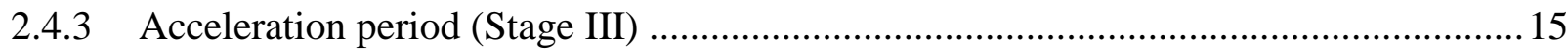

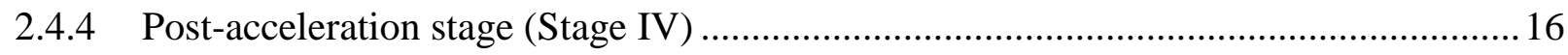

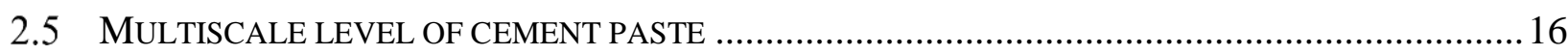




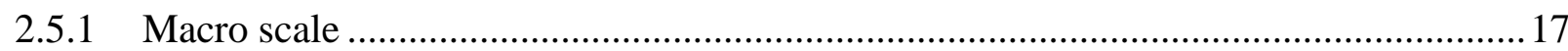

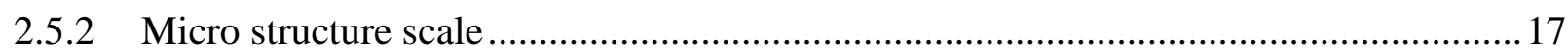

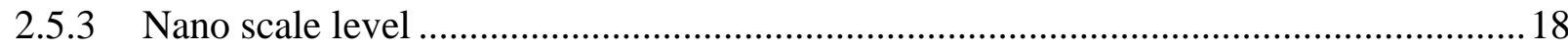

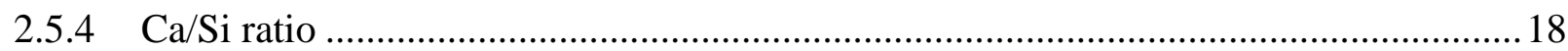

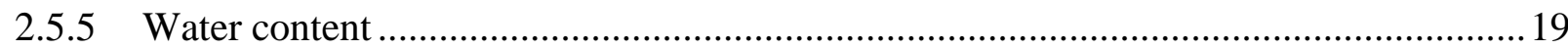

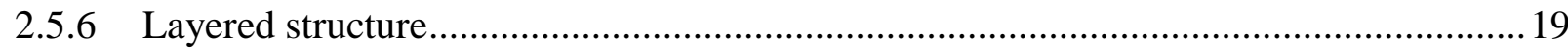

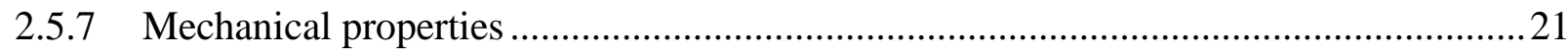

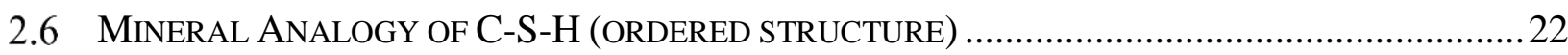

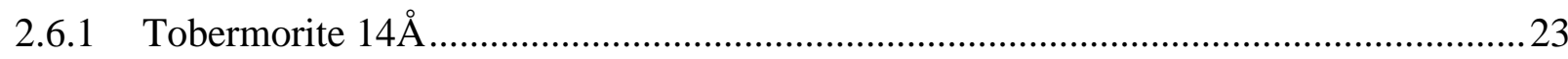

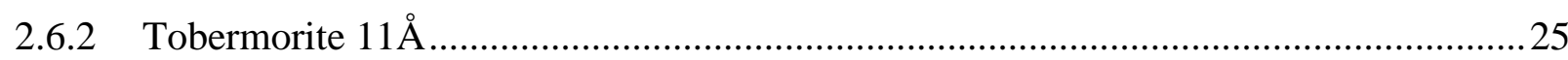

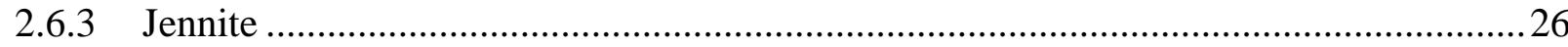

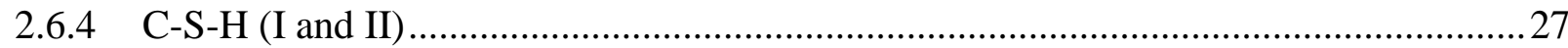

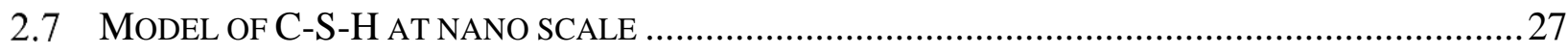

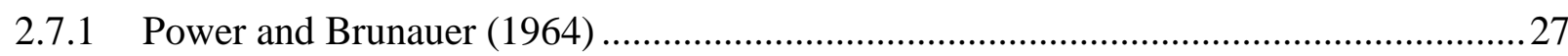

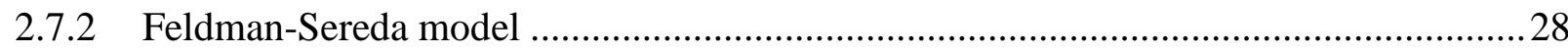

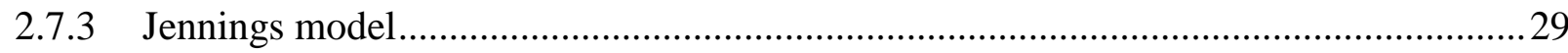

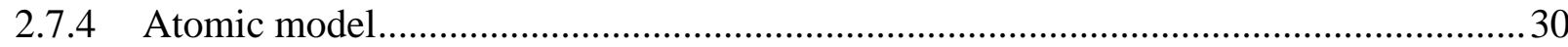

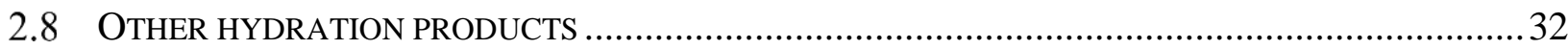

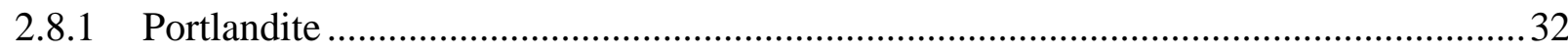

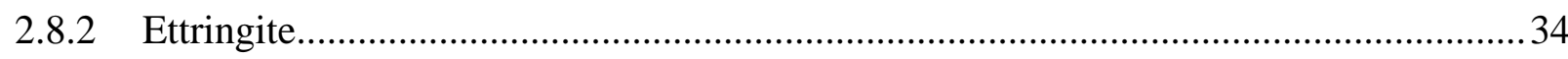

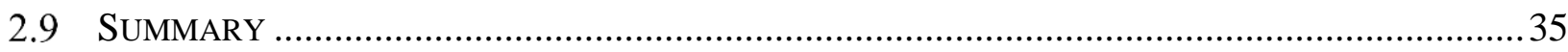

CHAPTER 3 COMPUTATIONAL METHODS.......................................................................38

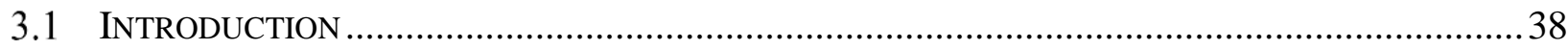

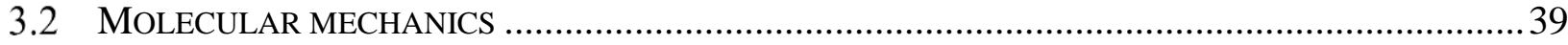

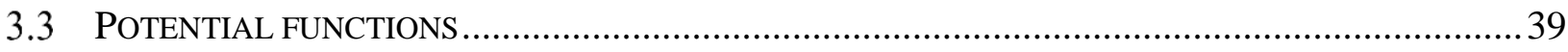

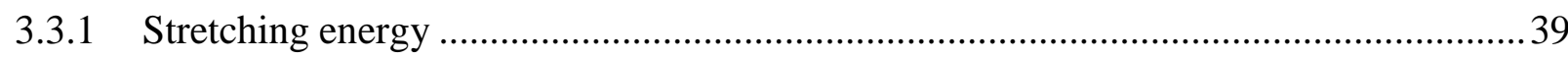

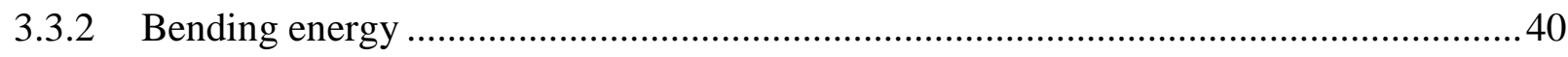

3.3.3 Torsion energy ................................................................................................... 41

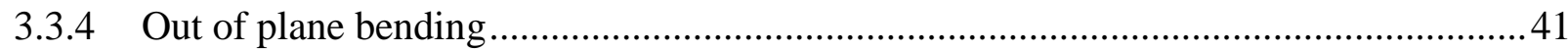

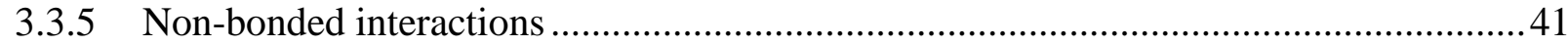

3.3.6 Electrostatic interactions (long range) ………........................................................... 42

3.3.7 Van der Waals interactions (short Range) .................................................................. 43 


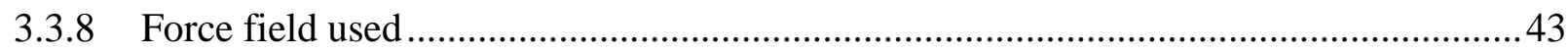

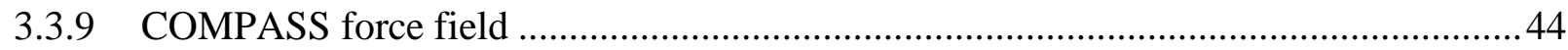

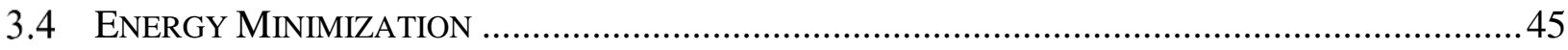

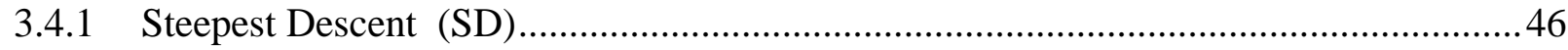

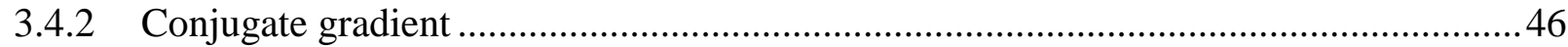

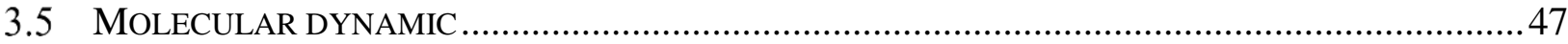

3.5.1 Integration of equation of motion (algorithms) ......................................................... 49

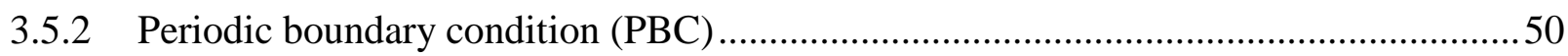

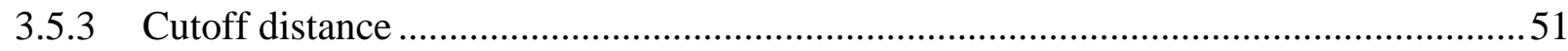

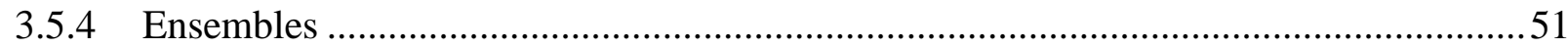

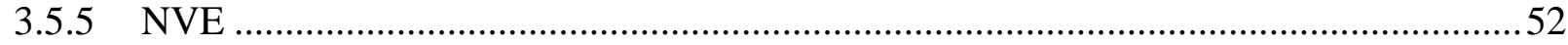

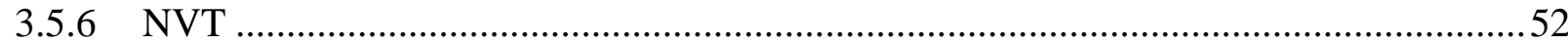

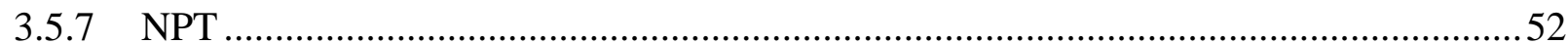

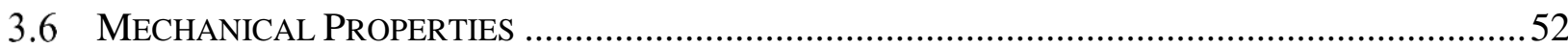

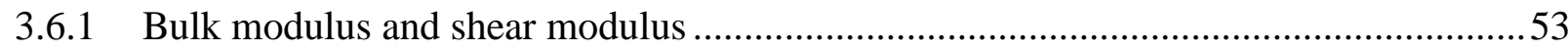

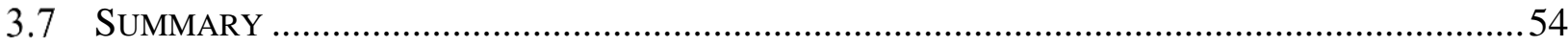

CHAPTER 4 ELASTIC PROPERTIES OF MAJOR CLINKER PHASES .............................56

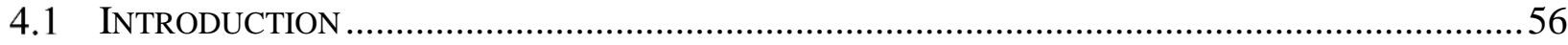

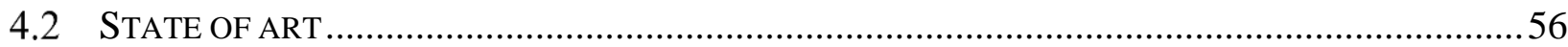

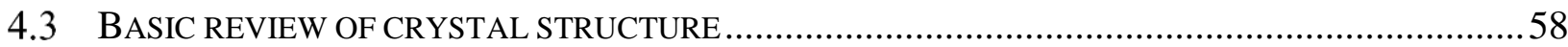

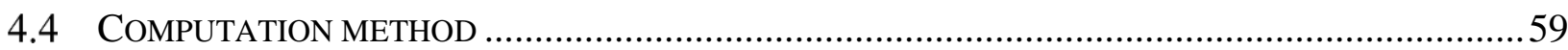

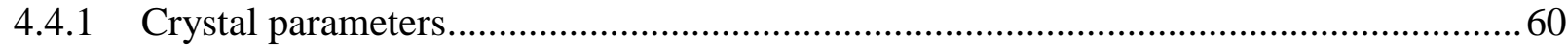

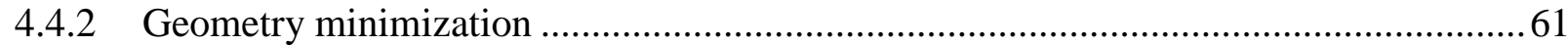

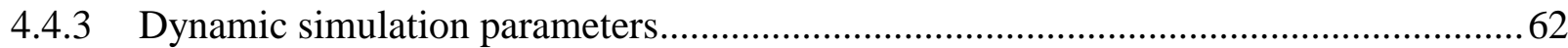

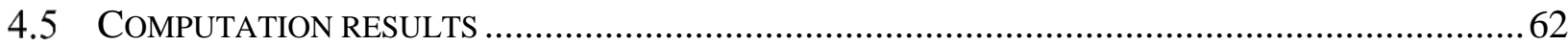

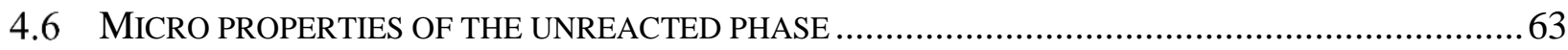

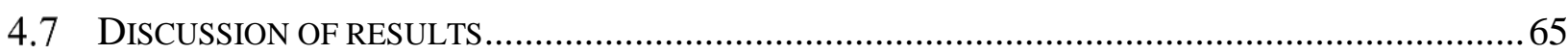

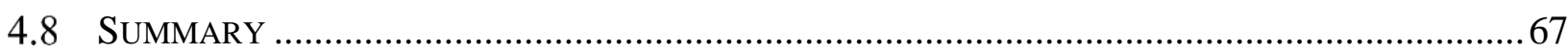

\section{CHAPTER 5 ELASTIC PROPERTIES OF HYDRATION PRODUCTS ................................69}

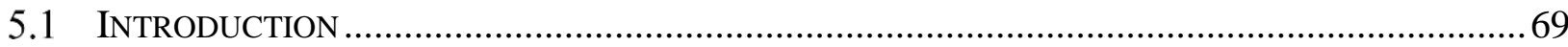

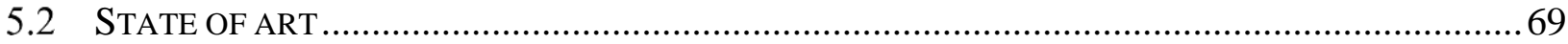




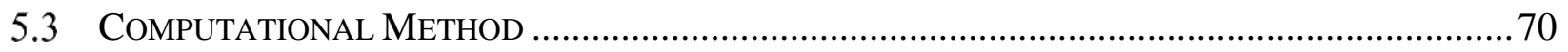

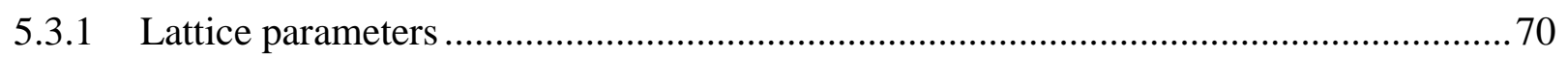

5.3.2 Geometry optimization parameters ………………................................................. 71

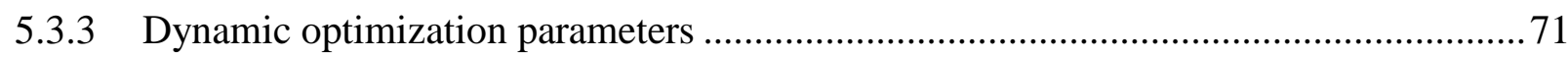

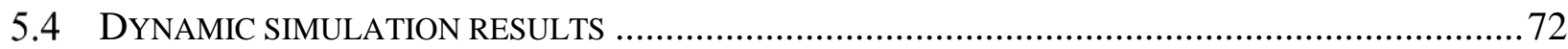

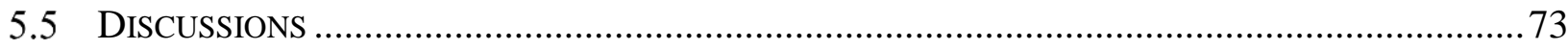

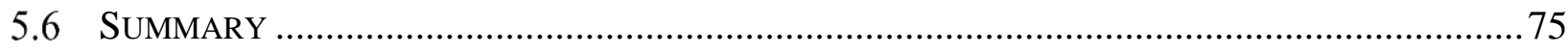

\section{CHAPTER 6 ELASTIC PROPERTIES OF ANALOG MINERALS..........................................76}

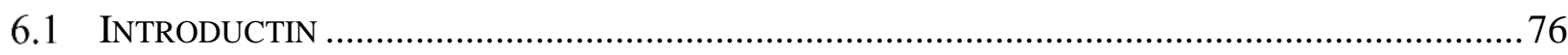

6.2 STATE OF ART OF ELASTIC PROPERTIES OF TOBERMORITE AND JENNITE...................................76

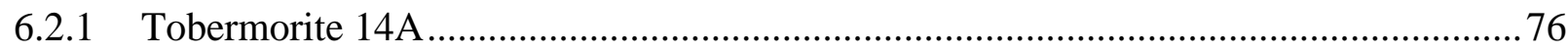

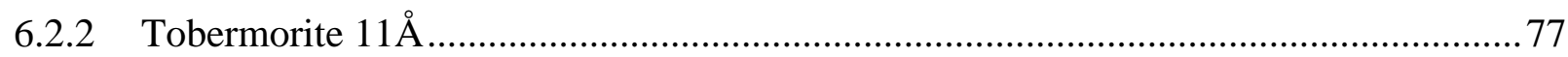

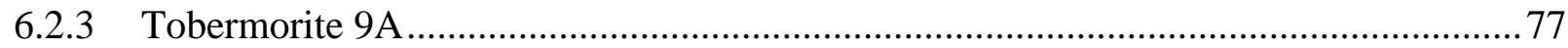

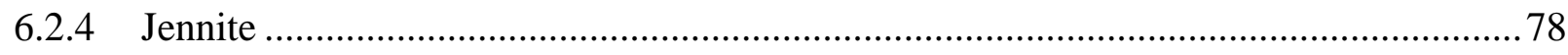

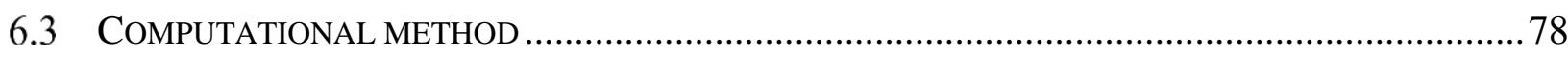

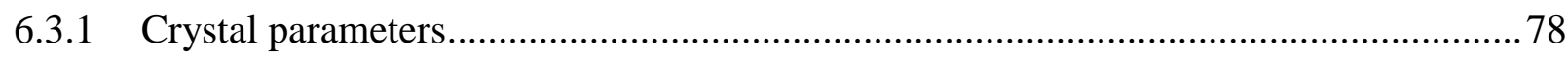

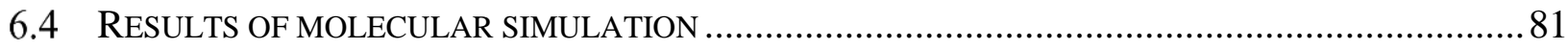

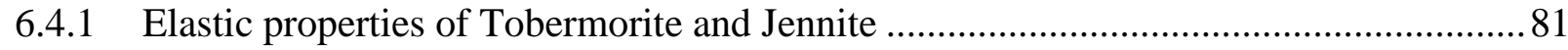

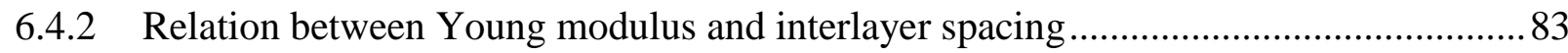

6.4.3 Relation between Young modulus and C/S, density and water ..................................... 84

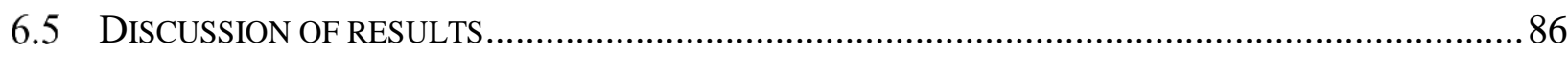

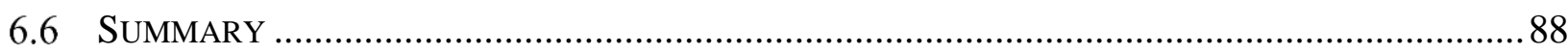

\section{CHAPTER 7 C-S-H GEL ELASTIC PROPERTIES .......................................................................90}

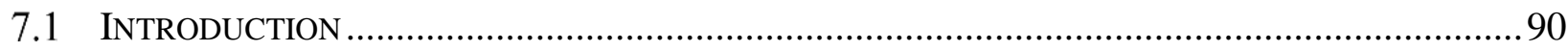

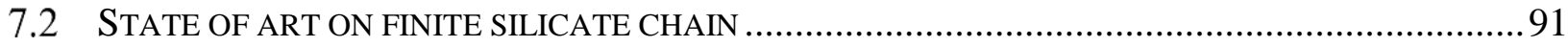

7.3 COMPUTATIONAL METHOD OF FINITE SILICATE CHAIN OF TOBERMORITE 14A AND JENNITE. 92

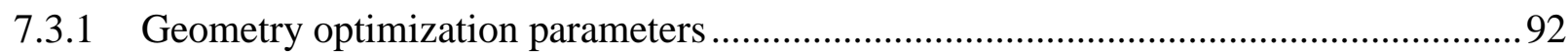

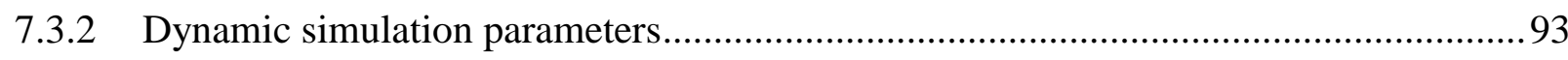

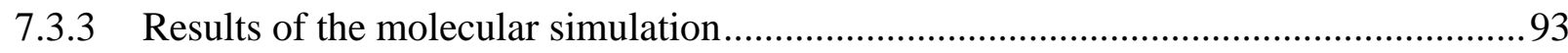

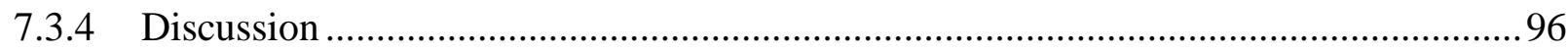

7.4 THE ACCOUNTING OF POROSITY FOR TOBERMORITE 14A AND JENNITE..................................99 
7.4.1 Mechanical properties of porous materials at microscale level ................................ 97

7.4.2 Elastic properties of C-S-H - low density (LD) and high density (HD) C-S-H .......... 98

7.4.3 Elastic properties of analog C-S-H minerals accounting for porosity .......................99

7.4.4 Elastic properties of Tobermorite 14A and Jennite at LD zone............................... 100

7.4.5 Elastic properties of Tobermorite 14A and Jennite at HD zone ............................. 101

7.5 RELATIONSHIP BETWEEN YOUNG MODULUS AND PACKING FACTORS .............................. 103

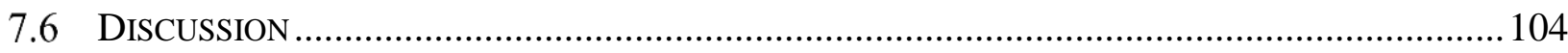

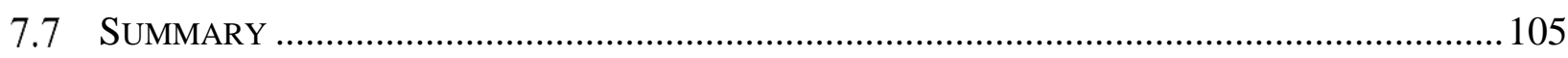

CHAPTER 8 GENERAL CONCLUSIONS .............................................................................108

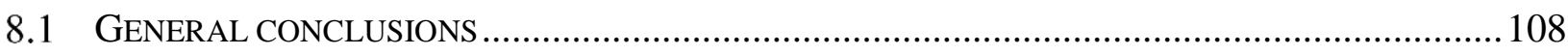

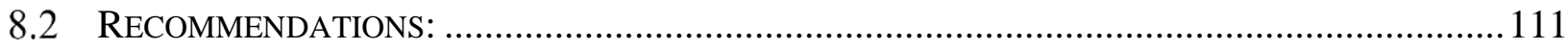

Appendixes: Input parameters to Materials Studio software and elastic properties...........112

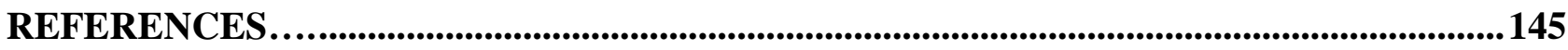




\section{List of Tables}

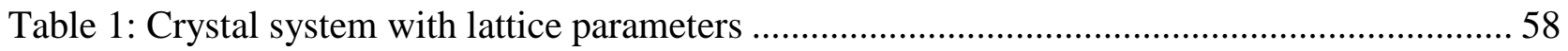

Table 2: Unit cell parameters for different clinker phases ............................................................. 61

Table 3: Unit cell parameters for different clinker phases .............................................................. 61

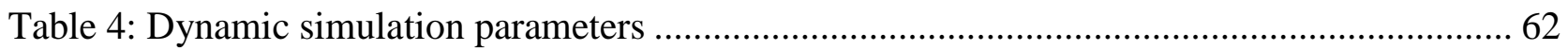

Table 5 : Elastic properties of cement clinker: bulk modulus, shear modulus and Young modulus, Poisson

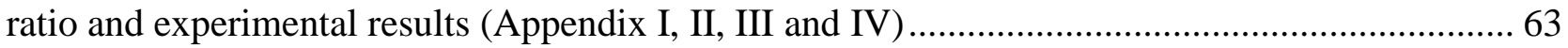

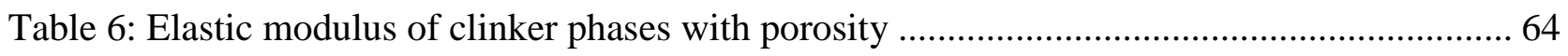

Table 7: Lattice parameters for Portlandite and Ettringite.............................................................. 71

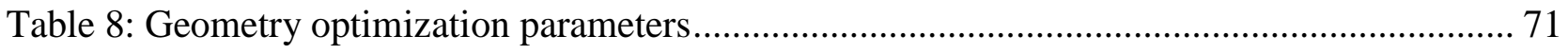

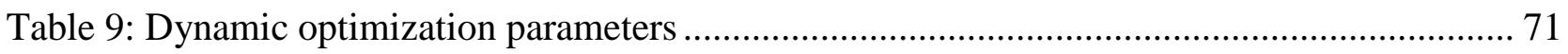

Table 10: MD simulation results of Portlandite and Ettringite (Appendix VI) ................................ 72

Table 11: Lattice parameters of different models of Tobermorite and Jennite................................ 79

Table 12: Geometry minimization parameters............................................................................. 79

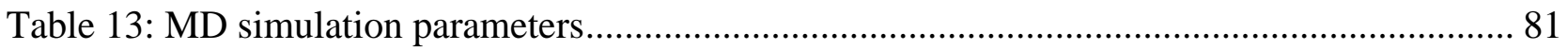

Table 14: Elastic properties of Tobermorite $9 \AA$ and Tobermorite $11 \AA$ (Appendix VII, VIII) ......... 81

Table 15: Elastic properties of Tobermorite 14£ and Jennite (Appendix IX, X)............................ 82

Table 16: Properties of different types of Tobermorite and Jennite (C/S, density, W/Ca and W/Si and

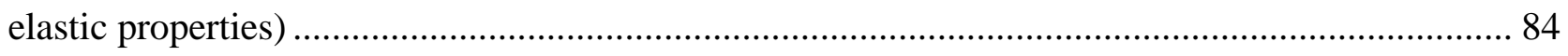

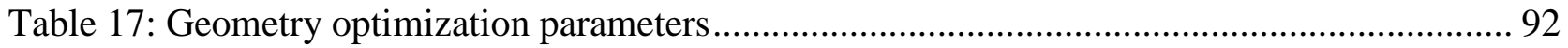


Table 18: Dynamic simulation parameters

Table 19: Elastic properties of Tobermorite and Jennite with different silicate chain lengths (Appendix

IX, X, XI, XII)

94

Table 20: Elastic properties of Tobermorite and Jennite with different chain length at LD zone $(\mathrm{P}=0.36)$

100

Table 21: Elastic properties of Tobermorite and Jennite with different chain length at HD zone $(\mathrm{P}=0.26)$ 


\section{List of Figures}

Figure 1 : Unit cell of $\mathrm{C}_{3} \mathrm{~S}$ where $\mathrm{Ca}$ is presented in green, silica in orange, oxygen in red, and the white line represents the unit cell 7

Figure 2: X-ray powder data $(\mathrm{CuK} \alpha)$ diagrams for triclinic of $\mathrm{C}_{3} \mathrm{~S}$ 8

Figure 3: Unit cell of $\mathrm{C}_{2} \mathrm{~S}$ where $\mathrm{Ca}$ is presented in green, silica in orange, oxygen in red, and the white line represents the unit cell 9

Figure 4: X-ray powder data $(\mathrm{CuK} \alpha)$ diagrams for monoclinic of $\beta-\mathrm{C}_{2} \mathrm{~S}$. 9

Figure 5: Unit cell of $\mathrm{C}_{3} \mathrm{~A}$ where $\mathrm{Ca}$ is presented in green, aluminum in magenta, oxygen in red, and the white line represents the unit cell. 10

Figure 6: $\mathrm{X}$-ray powder data $(\mathrm{CuK} \alpha)$ diagrams for cubic of $\mathrm{C}_{3} \mathrm{~A}$ Ferrite $\mathrm{C}_{4} \mathrm{AF}$ 10

Figure 7: Unit cell of $\mathrm{C}_{4} \mathrm{AF}$ where $\mathrm{Ca}$ is presented in green, aluminum in magenta, oxygen in red, iron in violet, and the white line represents the unit cell 11

Figure 8: X-ray powder data $(\mathrm{CuK} \alpha)$ diagrams for triclinic of $\mathrm{C}_{4} \mathrm{AF}$ 11

Figure 9: Top figure shows concentration of $\mathrm{Ca}^{2+}$ in solution with time; bottom figure shows different stages of hydration as a function of time (Barnes, 1983) 15

Figure 10: Structural hierarchy in concrete 16

Figure 11: $\mathrm{Ca} / \mathrm{Si}$ ratio frequency histogram for $\mathrm{C}-\mathrm{S}-\mathrm{H}$ in Portland cement paste aged 1 day to 3 years (TEM microanalysis) (Richardson, 1999) 19

Figure 12: Different connections between tetrahedral $\mathrm{SiO}_{4}$ 20

Figure 13: TMS-GPC chromatogram of white Portland cement paste hydrated for 3 months at $20^{\circ} \mathrm{C}$;

$\mathrm{W} / \mathrm{C}=0.55$ 
Figure 14: Mean silicate chain relationship with $\mathrm{Ca} / \mathrm{Si}$ ratio (Hou, 2014)

Figure 15: Relationship between packing density and indentation modulus (Pellenq et al., 2009) 22

Figure 16: Idealized chemical structure of Tobermorite 14A (Hewlett, 2004) 23

Figure 17: View of bridging tetrahedral and Pair tetrahedrals of Tobermorite (Shahsavari, Buehler, Pellenq, \& Ulm, 2009) 24

Figure 18: Unit cell of Tobermorite 14A where Ca is presented in green, silicon in orange, oxygen in red, hydrogen in white, and the white line represents the unit cell 24

Figure 19: X-ray diffraction of Tobermorite 14A....... 25

Figure 20: Hamid crystal structure showing linear silicate. 25

Figure 21: Merlino crystal structure showing silicate tetrahedral connected at the interlayer 26

Figure 22: Unit cell of Jennite where $\mathrm{Ca}$ is presented in green, silicon in orange, oxygen in red, hydrogen in white, and the white line represents the unit cell 26

Figure 23: X-ray diffraction of Jennite 27

Figure 24: Schematic representation of Powers model (Feldman and Sereda, 1968) 28

Figure 25: Schematic representation of Feldman-Sereda model (Feldman and Sereda, 1968)..... 29

Figure 26: a) Basic building block, b) Globules, c) LD C-S-H. d) HD C-S-H and accessibility of $\mathrm{N}_{2}$ to pores (Jennings, 2000) 30

Figure 27: Silicate chain of Tobermorite showing the probable position of $\mathrm{H}$ atoms and omission of bridging tetrahedrals (Taylor, 1986) 31

Figure 28: C/S ratio vs chain length (Taylor H. F., 1986) 32

Figure 29: Unit cell Portlandite where $\mathrm{Ca}$ is presented in green, oxygen in red, hydrogen in white and the white line represents the unit cell. 33 
Figure 30: X-ray diffraction of Portlandite

Figure 31: Unit cell Ettringite where $\mathrm{Ca}$ is presented in green, oxygen in red, hydrogen in white, aluminum in magenta and the white line represents the unit cell 34

Figure 32: X-ray diffraction of Ettringite 35

Figure 33: Bond stretching 40

Figure 34: Angle bending 40

Figure 35: Bond torsion 41

Figure 36: Out of plane 41

Figure 37: Electrostatic interactions 42

Figure 38: The Lennard-Jones potential (Leach, 2001) 43

Figure 39: A schematic one-dimensional energy surface, minimization method moving downhill to nearest minimum (Leach, 2001) 45

Figure 40: Minimization path followed by steepest-descents approach for simple quadratic function (Materials Studio) 46

Figure 41: Minimization comparison between Steepest-Descents and Conjugate gradient method (Materials Studio) 47

Figure 42: Flow chart of MD algorithm. 49

Figure 43 : Graphical representation of steps followed by Verlet algorithm (Allen and Tildesley, 1987) 50

Figure 44: Periodic boundary conditions in molecular dynamics (Allen and Tildesley, 1987) ...... 51

Figure 45: Elastic modulus map using nano indentation (Mondal, 2008) 57

Figure 46: Crystal structure showing angles in the cell 58 
Figure 47: Potential energy, kinetic energy, non-bonded energy and total energy fluctuation during equilibration phase 60

Figure 48: Fluctuation of temperature and running average Temperature during equilibration phase 60

Figure 49: Elastic moduli as a function of the relative porosity of clinker phases 65

Figure 50 : Relationship between elastic modulus and interlayer spacing of Tobermorite family . 84

Figure 51: Young Modulus vs. C/S ratio Figure 52 : Young modulus vs. density 85

Figure 53: Young modulus vs. W/Ca ratio

Figure 54: Young modulus vs. W/Si ratio .85

Figure 55: Tobermorite structure with infinite and finite chain (bridging silicate removed) 91

Figure 56: Relationship between Young modulus and silicate chain length of Tobermorite and Jennite 95

Figure 57: Relationship between Young modulus and density of Tobermorite, Tobermorite T2, Jennite and Jennite $\mathbf{J} 2$. 96

Figure 58: Frequency density as function of indentation modulus (10x10) indentation tests on a grid of spacing $10 \mu \mathrm{m}$ ) Constantinides and Ulm (2006), PDF; probability density function 99

Figure 59: Location of indentation modulus of different Tobermorite 14A and Jennite samples in experimental C-S-H phases, $(\mathrm{P}=0.36 \%)$. 101

Figure 60: Location of indentation modulus of different Tobermorite 14A and Jennite samples in the experimental C-S-H phases, $(\mathrm{P}=0.26 \%)$. 103

Figure 61: Young modulus vs. packing factor. 103 


\section{Chapter 1 Introduction}

Over the past few years, there has been a growing interest and focus on sustainable buildings. Materials are now evaluated for environmental impact associated with their manufacturing and transportation. Concrete is the most commonly used construction material, with a $4 \%$ annual increase in cement production due to demand (Kusuma, Budidarmawan, \& Susilowati, 2015). However, the construction of city infrastructure, which is the base for economic growth and job creation, results in a release of significant amounts of $\mathrm{CO}_{2}$ and greenhouse gas emissions. One way to decrease environmental impact and contribute to sustainable development is to improve concrete quality, in which cement is the primary ingredient. Improved concrete quality increases the lifespan of structures, reducing environmental impact and project cost (Kusuma et al., 2015). For instance, improved concrete quality with low standard deviation $(\mathrm{S}=2.4 \mathrm{MPa})$ has a $41 \%$ smaller carbon footprint than low quality and high standard deviation ( $\mathrm{S}=8.6 \mathrm{MPa}$ ) concrete (Kusuma, et al., 2015).

Nanotechnology can be used to develop a new material with higher performance and sustainability. Nanotechnology was introduced by physicist Richard Feynman in his 1959 lecture, (Sanchez \& Sobolev, 2010) in which he suggested the possibility of manipulating atoms and molecules to create new materials with improved properties. Although nanotechnology has different meanings from country to country, all definitions agree that it deals with elements at a very small scale, generally less than $100 \mathrm{~nm}$ (Sanchez \& Sobolev, 2010). The concept of nanotechnology was well developed in the 1990s in chemistry, biology, physics and other fields. It has two approaches: top down and bottom up. In the top down approach, bulk material is reduced to nanoscale level without changing the properties of the material. The purpose of this approach is to create small devices and materials at 
nanoscale level such as computer chips, nano silica, carbon nanotubes, nano wire and nano powder. In the bottom up approach - also called "molecular nanotechnology" (Sanchez \& Sobolev, 2010) - the material is made by assembling atoms and molecules. Although molecular nanotechnology has made advances in fields such as medicine, energy, biotechnology and information technology, it is still not widely used in cementitious material. The major progress in its use in cement paste has been to understand the structure and mechanical properties of key cement paste components, as well as the source of forces between particles.

The binding element in concrete is the Calcium Silicate Hydrate (C-S-H). When seen from the bottom up approach, it is composed of atoms that bind together through different types of interaction such as chemical bond, Coulombic, van der Waal interactions and others. The purpose of the bottom up approach, which deals with manipulation of the structure at nano scale, is to develop a new generation of materials with high mechanical properties, durability, self-sensing, self-healing and self-cleaning properties.

The purpose of computation is to predict the behavior and the properties of the material in different conditions such as high temperature (melting), high pressure and others, with the use of physics and chemistry theories and principles.

Material computation is also helpful when the experimental and computational results support each other. Results of computational methods can help predict the behavior and engineering properties of cement paste and aid in the development of new materials. It is also beneficial because it reduces the number of experiments needed when developing a new material, and its results are used for comparison with experimental results.

There are two main approaches in material computation to model interaction between atoms: the empirical approach and quantum mechanics (ab initio). In the empirical approach, a set of simple equations (force field) describes the interactions between atoms. The quantum mechanics approach is 
based on the solution of the Schrödinger wave equations of all electrons in the system. Four methods are derived from these two approaches: lattice energy modeling, calculation of lattice dynamics, Monte Carlo simulation and molecular dynamic simulation (MD).

Lattice energy modeling, calculation of lattice dynamics and Monte Carlo simulation all require timeconsuming, expensive and rigorous computation with very powerful computers. Therefore, for this thesis, an empirical approach using MD simulation was chosen to speed simulation time, better understand the structure of materials at nanoscale level, predict and improve its properties at micro/macro scale level. Many efforts have been made to determine the structure of calcium silicate hydrate. However, because C-S-H has an amorphous structure that changes over time, its structure is not yet defined, which is the major challenge in cement paste modeling. Experimental methods using NMR, XANS, nano indentation and other tools have provided some information about the structure of $\mathrm{C}-\mathrm{S}-\mathrm{H}$; it is a layered structure composed of short-range crystals. However, some parameters can't be determined experimentally. The quantum mechanics computational method (especially the ab-initio method) offered additional information, including the cohesion forces between C-S-H and the relation between the C-S$\mathrm{H}$ structure and Tobermorite family and Jennite, which cannot be determined by experimental tools.

In addition to the similarity with Tobermorite and Jennite, Pellenq et al. (2009) proposed a C-S-H model that can bridge the gap between theoretical and experimental results. However, the proposed structure is not well developed and has limitations. Shahsavari et al. (2009) carried out a dynamic simulation using Density Function Theory (DFT) method using Tobermorite 9A, 11A and 14A. Those authors calculated a Young modulus of 95.06 GPa, 82.82GPa and 51.9 GPa for Tobermorite 9A, Tobermorite 11A and Tobermorite 14A, respectively. Dolado et al. (2007) studied the polymerization of the silicate $(\mathrm{SiOH})_{4}$ in the presence of ions. They concluded that increased calcium content $(\mathrm{Ca} / \mathrm{Si})$ slows the polymerization of the silicate chain. Quantum mechanics methods such as ab initio and DFT are complex, expensive and 
time-consuming methods, and require robust computers, which make these methods difficult and reveal the need for further comprehensive and simplified studies on C-S-H simulation.

Using less complicated methods with regular computers and reduced simulation time to achieve reliable results is the principal goal of this study. This can be achieved by using molecular dynamic simulation based on Newton law. The force field used in the molecular dynamic simulation can be considered as the principal parameter that affect the results of the simulation. Among the force fields incorporated in the Materials Studio software (Materials Studio v7), COMPASS force field is an option for reliable results. COMPASS force field has been broadly used for different ranges of molecules and polymers. However, for cement paste components, and especially C-S-H, it has only been used in limited studies. In this study, COMPASS force field is used to determine the elastic properties of clinker phases and cement hydration products such as Portlandite, Ettringite and C-S-H. Special attention was given to C-S-H and its analog minerals including the Tobermorite family and Jennite.

Elastic properties are the main parameters that control the short-term deformation of any structure. For this reason, this research targeted elastic properties such as bulk modulus, shear modulus, Poisson ratio and Young modulus in the dynamic simulation. However, because of the direct relationship between the Young modulus and other elastic properties, most discussions of simulation results of analog $\mathrm{C}-\mathrm{S}-\mathrm{H}$ minerals are based on Young modulus values, as are discussions of the influence of different parameters (density, packing factor, W/Ca, W/Si, porosity) on the elastic properties of analog $\mathrm{C}-\mathrm{S}-\mathrm{H}$ minerals, and the comparison between the elastic properties in this study and those mentioned in previous literature. 


\section{Thesis outline:}

Chapter 2 covers the basic chemistry of cement and main clinker phases including $\mathrm{C}_{3} \mathrm{~S}, \mathrm{C}_{2} \mathrm{~S}, \mathrm{C}_{3} \mathrm{~A}$ and $\mathrm{C}_{4} \mathrm{AF}$. Hydration process and mechanism, the multiscale level of cement paste and the proposed models for C-S-H gel at its macro-, micro-, nano-scale and atomic-scale levels are also presented.

Chapter 3 is devoted to the computational method used in this thesis to predict the mechanical properties of the different phases of cement by using molecular mechanics to calculate the energy potential in the system. The chapter also covers the steps required for molecular modelling, including energy minimization, dynamic simulation and production run.

Chapter 4 deals with the results of the molecular dynamic simulation by Compass force fields of $\mathrm{C}_{3} \mathrm{~S}$, $\mathrm{C}_{2} \mathrm{~S}, \mathrm{C}_{3} \mathrm{~A}$ and $\mathrm{C}_{4} \mathrm{AF}$. The elastic properties of the clinker phases resulting from this study are also compared to the experimental results, and the effect of packing density on elastic modulus is discussed.

Chapter 5 covers the elastic properties of Portlandite and Ettringite, the major hydration products of cement reaction. It also includes a comparison between the results of this study and those in literature using other experimental and numerical methods.

Chapter 6 is devoted to the calculation of the elastic properties of analog C-S-H minerals such as the Tobermorite family and Jennite. The effects of C/S ratio, density and water on elastic modulus of analog C-S-H minerals are also discussed.

Chapter 7 is focused on the results of two tested methods to reduce the Young modulus of Tobermorite 14A and Jennite to be closer to that of $\mathrm{C}-\mathrm{S}-\mathrm{H}$. The effects of $\mathrm{C} / \mathrm{S}$ ratio, silicate chain and porosity on Young modulus results are also discussed.

Chapter 8 summarizes the general conclusions of this study and offers recommendations for future research. 


\section{Chapter 2 Chemistry of Portland cement}

\subsection{Introduction}

Cement is a substance used to bind sand and aggregates. The most commonly used variety is Portland cement; when mixed with water, chemical reactions occur between the components of cement and water to produce a hardened paste. The reaction that causes the setting and hardening of the paste is called a hydration reaction. The Egyptians were the first to use gypsum mortar - made from the calcination of impure gypsum at $130^{\circ} \mathrm{C}$ (Mindess and Young, 1981) - as binding material. When gypsum is mixed with a small quantity of water, it sets and hardens. However, it is a soluble mortar and cannot harden under water. Lime mortar was also used by the Egyptians; it was prepared by calcining limestone at $1000^{\circ} \mathrm{C}$ (Mindess et al., 2003). Greeks and Romans also used volcanic ash (Pozzolan). When ground and mixed with lime, sand and water, it creates a mortar that is resistant to water when set and hardened. However, most Romans knowledge - such as burning lime and adding Pozzolan - was lost during the Middle Ages. It was not until 1756, when John Smeaton conducted experiments with lime and pozzolan, he found that the best limestone is one containing a high proportion of clay mineral. Another remarkable discovery was made by Joseph Aspdin, who patented Portland cement in 1824; it is prepared by calcining and mixing ground limestone with fine clay in a kiln (Mindess et al., 2003) then finely grinding it to be used as cement. 


\subsection{Clinker Phases}

\subsubsection{Alite, C3S}

Alite $\left(\mathrm{Ca}_{3} \mathrm{SiO}_{5}\right)$ is the impure form of $\mathrm{C}_{3} \mathrm{~S}$. It is a highly reactive compound because of its high calcium content, and a major constituent of Portland cement clinker, with a range of 50 to $90 \%$ of clinker phase (Barnes and Bensted, 1983). Tricalcium silicate is a stable polymorph at temperatures between $1250^{\circ}-2150^{\circ} \mathrm{C}$. It becomes unstable at temperatures lower than $1250^{\circ} \mathrm{C}$ but preserves its stability state with rapid cooling. Slow cooling can change Alite structure to Belite or lime $(\mathrm{CaO})$ (Barnes and Bensted, 1983). As the temperature in the clinker changes, Alite undergoes different states as shown:

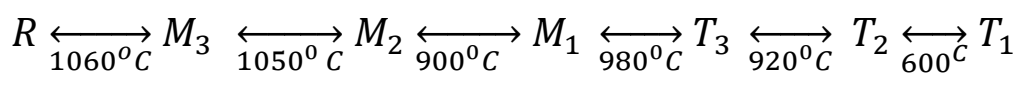

(T: Triclinic, M: Monoclinic and R: rhombohedral)

The crystal structures of these phases are similar with small distortion in $\mathrm{SiO}_{4}{ }^{4-}$ tetrahedral. $\mathrm{C}_{3} \mathrm{~S}$ has a crystalline structure composed of $\mathrm{Ca}^{2+}, \mathrm{SiO}_{4}{ }^{4-}, \mathrm{O}^{2-}$ ions with a $\mathrm{C} / \mathrm{S}$ ratio of $3: 1$. Figure 1 shows the unit cell of triclinic $\mathrm{C}_{3} \mathrm{~S}$.

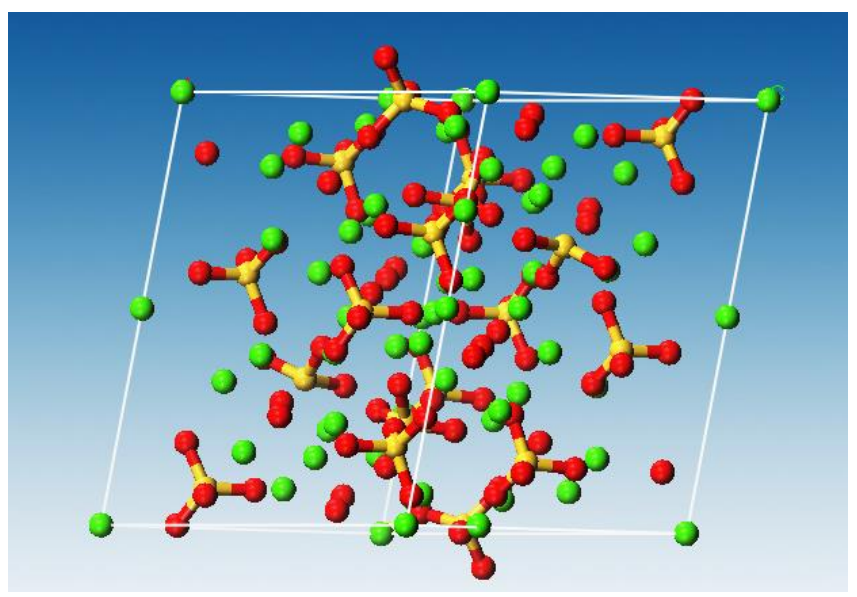

Figure 1 : Unit cell of $\mathrm{C}_{3} \mathrm{~S}$ where $\mathrm{Ca}$ is presented in green, silica in orange, oxygen in red, and the white line represents the unit cell

In industrial clinker, $\mathrm{C}_{3} \mathrm{~S}$ is mixed with foreign ions such as $\mathrm{Mg}^{2+}, \mathrm{Al}^{3+}$ and $\mathrm{Fe}^{3+}$, which are present in the raw mix. These impurities prevent the conversion of $\mathrm{C}_{3} \mathrm{~S}$ to $\mathrm{T}_{1}$, which is an unstable phase, and 
stabilize at high temperature such as $\mathrm{M}_{1}$ or $\mathrm{M}_{3}$. These impurities also prevent Alite from decomposing into dicalcium silicate $\mathrm{C}_{2} \mathrm{~S}$ or lime $(\mathrm{CaO})$ (Barnes and Bensted, 1983). The X-ray diffraction of triclinic $\mathrm{C}_{3} \mathrm{~S}$ is shown in Figure 2.

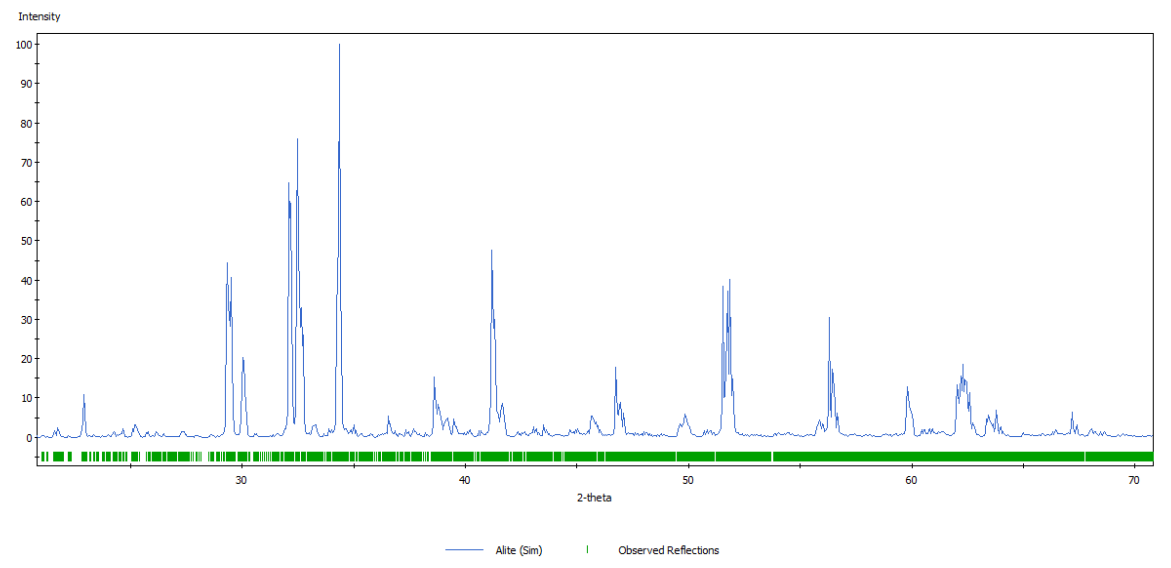

Figure 2: X-ray powder data $(\mathrm{CuK} \alpha)$ diagrams for triclinic of $\mathrm{C}_{3} \mathrm{~S}$

\subsubsection{Belite, $\mathrm{C}_{2} \mathrm{~S}$}

Belite $\left(\mathrm{Ca}_{2} \mathrm{SiO}_{4}\right)$, the impure form of $\mathrm{C}_{2} \mathrm{~S}$, is the second important clinker phase. It is also the largest, constituting 10 to $40 \%$ of the total clinker phase (Barnes and Bensted, 1983). Its hydration provides a similar strength as Alite, with a long period of hydration. It can be found in five types of polymorphs $\left(\alpha, \alpha_{\mathrm{H}}, \alpha^{\prime}{ }_{\mathrm{L}}, \beta\right.$ and $\left.\gamma\right)$, which differ in terms of structure and hydraulic properties. During cooling, $\alpha$ $\mathrm{C}_{2} \mathrm{~S}$ goes through these different polymorphs before transforming to $\beta-\mathrm{C}_{2} \mathrm{~S}$ at $630^{\circ} \mathrm{C}$. The $\alpha^{\prime} \mathrm{H}$ polymorph is the more hydraulic form of Belite; in contrast, the $\gamma$ polymorph is not reactive which can cause the disintegration (dusting) and cracking of the clinker (Barnes and Bensted, 1983). If the $\beta-\mathrm{C}_{2} \mathrm{~S}$ is not stabilized during cooling by the inclusion of impurities, $\gamma-\mathrm{C}_{2} \mathrm{~S}$ is less dense and can cause cracking. The $\beta-\mathrm{C}_{2} \mathrm{~S}$ can be stabilized at room temperature by doping impurities such as boric oxide, phosphorus pentoxide and magnesia. Belite can be formed in a number of ways, but is primarly formed with the reaction of lime and silicon. However, it can also be formed from Alite (Barnes and Bensted, 1983).

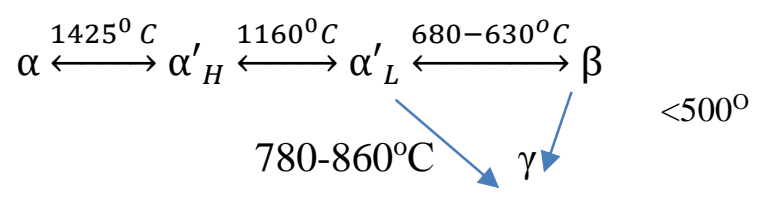


$C_{3} S \leftrightarrow C_{2} S+C$ (Barnes \& Bensted, 1983)

$\mathrm{H}$ : high temperature; L: low temperature

The crystal structure of all types of Belite is composed of $\mathrm{SiO}_{4}{ }^{4-}$ tetrahedrals and $\mathrm{Ca}^{2+}$. The arrangements of these ions are similar, with some differences in the $\gamma-C_{2} S$ (Taylor, 1997). The $\mathrm{C}_{2} \mathrm{~S}$ structure differs from Alite by the absence of $\mathrm{O}^{2-}$. Figures 3 and 4 show the unit cell of monoclinic $\beta-\mathrm{C} 2 \mathrm{~S}$ and the X-ray diffraction of $\beta-\mathrm{C} 2 \mathrm{~S}$.

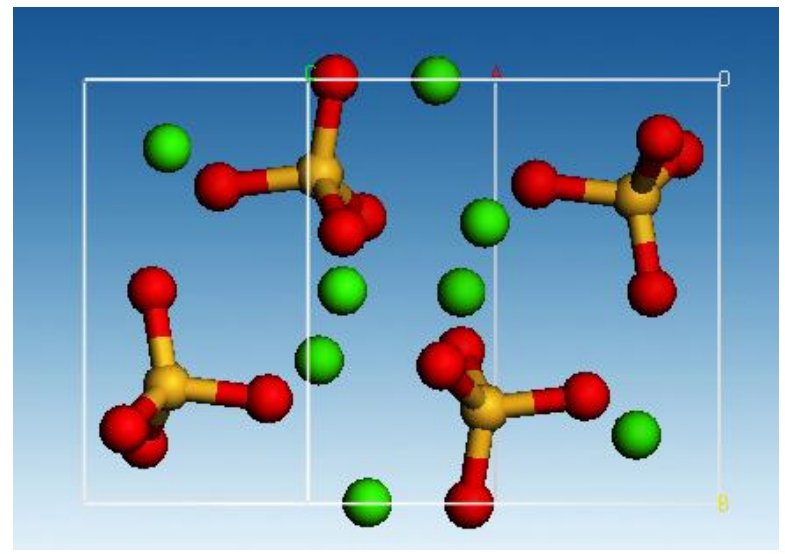

Figure 3: Unit cell of $\mathrm{C}_{2} \mathrm{~S}$ where $\mathrm{Ca}$ is presented in green, silica in orange, oxygen in red, and the white line represents the unit cell

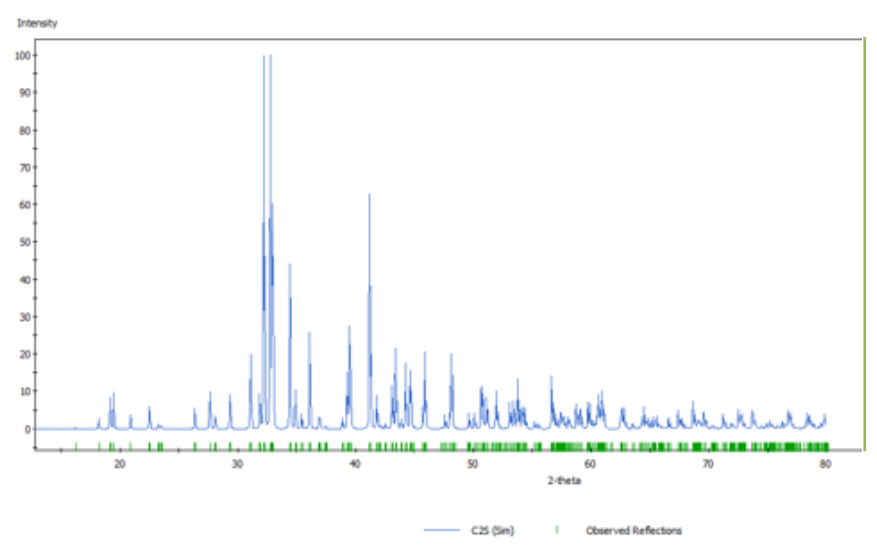

Figure 4: X-ray powder data $(\mathrm{CuK} \alpha)$ diagrams for monoclinic of $\beta-\mathrm{C}_{2} \mathrm{~S}$

\subsubsection{Tricalcium aluminate, $\mathrm{C}_{3} \mathrm{~A}$}

The third important component in ordinary Portland cement is tricalcium aluminate $\left(\mathrm{Ca}_{3} \mathrm{Al}_{2} \mathrm{O}_{3}\right.$ or $\mathrm{C}_{3} \mathrm{~A}$ ), which represents $10 \%$ of the clinker phase. Tricalcium aluminate does not appear in pure form, but rather, it is modified by the substitution of $\mathrm{Al}$ and $\mathrm{Ca}$ with $\mathrm{Na}^{+}$and $\mathrm{Si}^{+4}, \mathrm{Fe}^{3+}$ and $\mathrm{Mg}^{+2}$ or $\mathrm{K}^{+}$. The 
structure of tricalcium aluminate has a cubic form. However, due to the high quantity of alkalis in raw clinker, the $\mathrm{C}_{3} \mathrm{~A}$ can have an orthorhombic or monoclinic structure. The structure of $\mathrm{C}_{3} \mathrm{~A}$ is composed of rings of six $\mathrm{AlO}_{4}$ tetrahedrals and $\mathrm{Ca}^{2+}$ ions. The reactivity of these polymorphs depends on the quality of foreign ions existing in the lattice. Pure $\mathrm{C}_{3} \mathrm{~A}$ contains $62.3 \% \mathrm{CaO}$ and $37.7 \% \mathrm{Al}_{2} \mathrm{O}_{3}$. The total replacement of foreign ions is around $13 \%$ for the cubic and $20 \%$ for the orthorhombic (Taylor, 1997). The unit cell of $\mathrm{C}_{3} \mathrm{~A}$ and the X-ray diffraction of $\mathrm{C}_{3} \mathrm{~A}$ are shown in Figures 5 and 6, respectively.
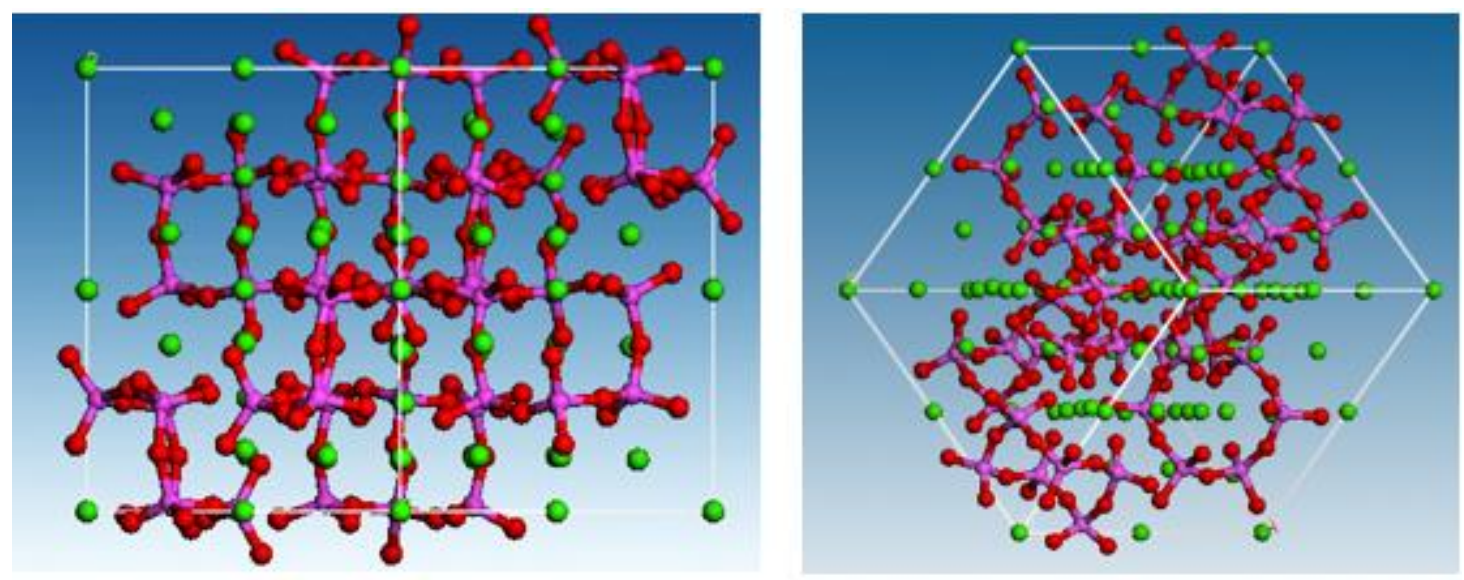

Figure 5: Unit cell of $\mathrm{C}_{3} \mathrm{~A}$ where $\mathrm{Ca}$ is presented in green, aluminum in magenta, oxygen in red, and the white line represents the unit cell

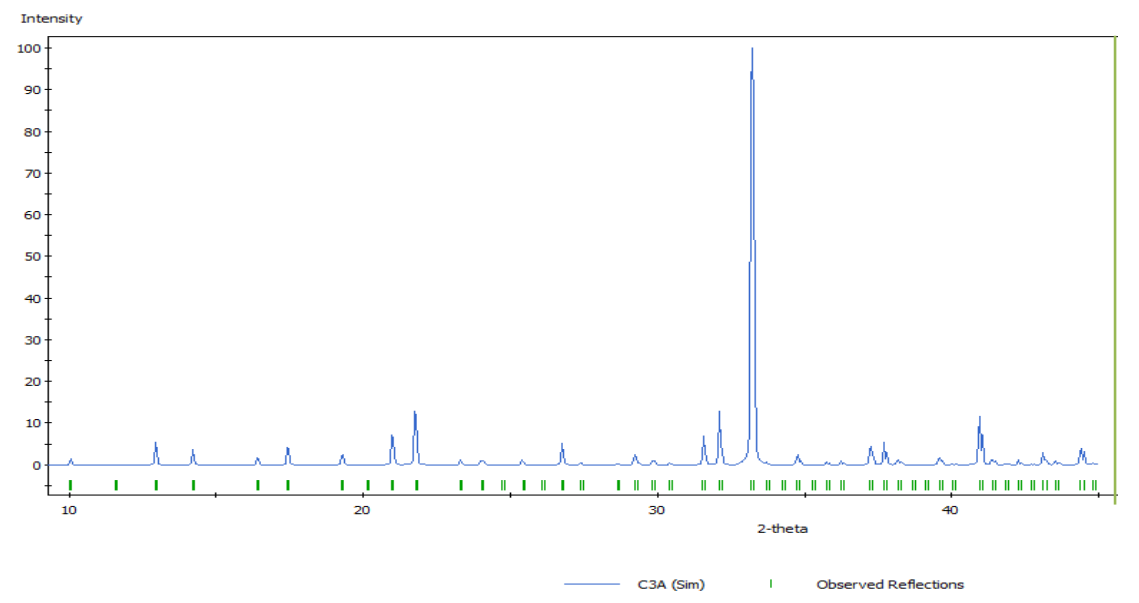

Figure 6: X-ray powder data $(\mathrm{CuK} \alpha)$ diagrams for cubic of $\mathrm{C}_{3} \mathrm{~A}$ Ferrite $\mathrm{C}_{4} \mathrm{AF}$

\subsubsection{Ferrite $\mathrm{C}_{4} \mathrm{AF}$}

The chemical formula of calcium aluminate ferrite can be written as $\mathrm{C}_{2}(\mathrm{~A}, \mathrm{~F})$. The structure of the ferrite phase is composed of $\mathrm{Ca}^{2+}$ ions surrounded by several oxygen ions, with $\mathrm{Al}^{3+}$ and $\mathrm{Fe}^{3+}$ 
distributed between octahedral and tetrahedral sites. In the ferrite phase, the $\mathrm{F}^{3+}$ ions may be substituted by $\mathrm{Mg}^{2+}, \mathrm{Si}^{4+}$ and $\mathrm{Ti}^{4+}$. Figures 7 and 8 show the unit structure on elevation (3D view) and the X-ray diffraction of the ferrite phase $\left(\mathrm{C}_{4} \mathrm{AF}\right)$.
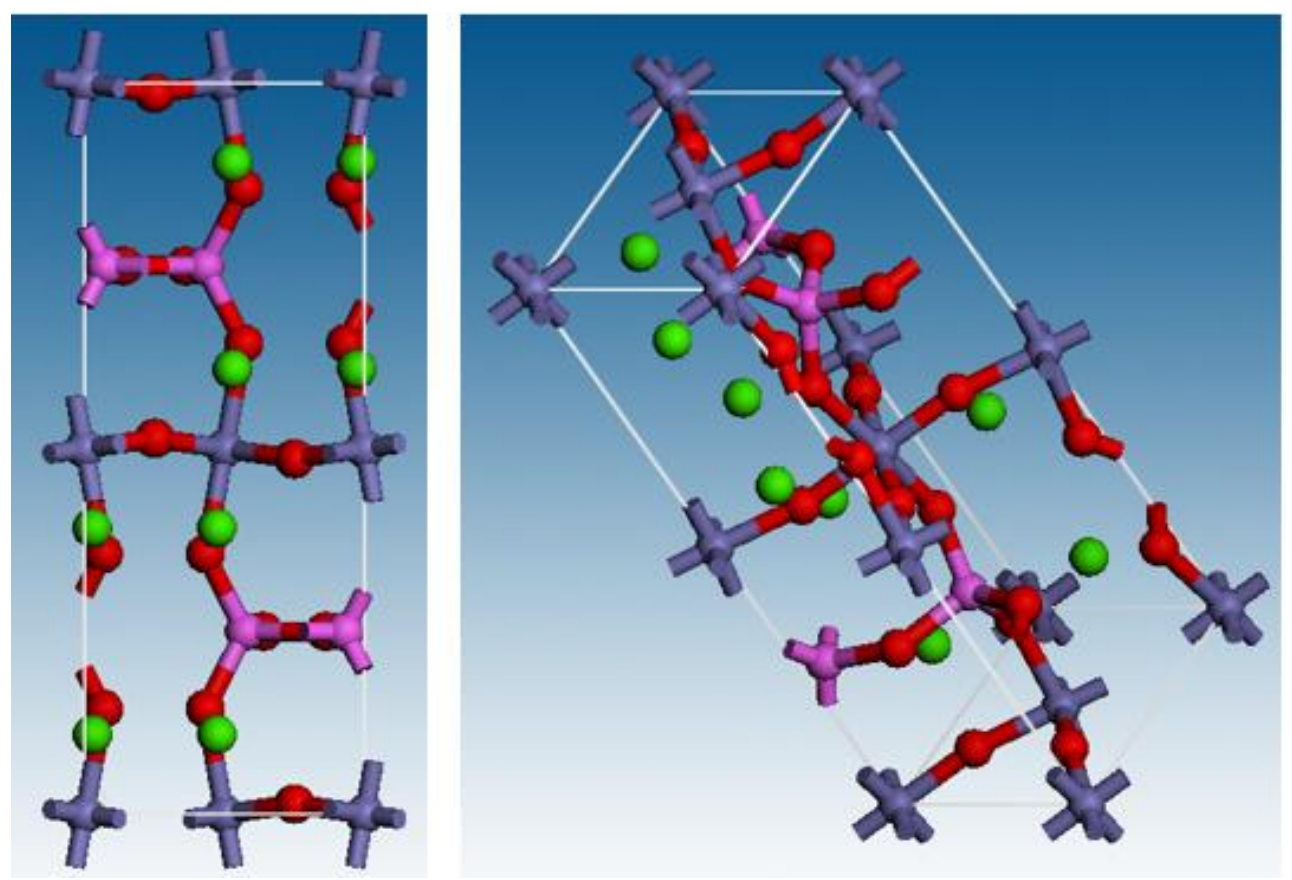

Figure 7: Unit cell of $\mathrm{C}_{4} \mathrm{AF}$ where $\mathrm{Ca}$ is presented in green, aluminum in magenta, oxygen in red, iron in violet, and the white line represents the unit cell

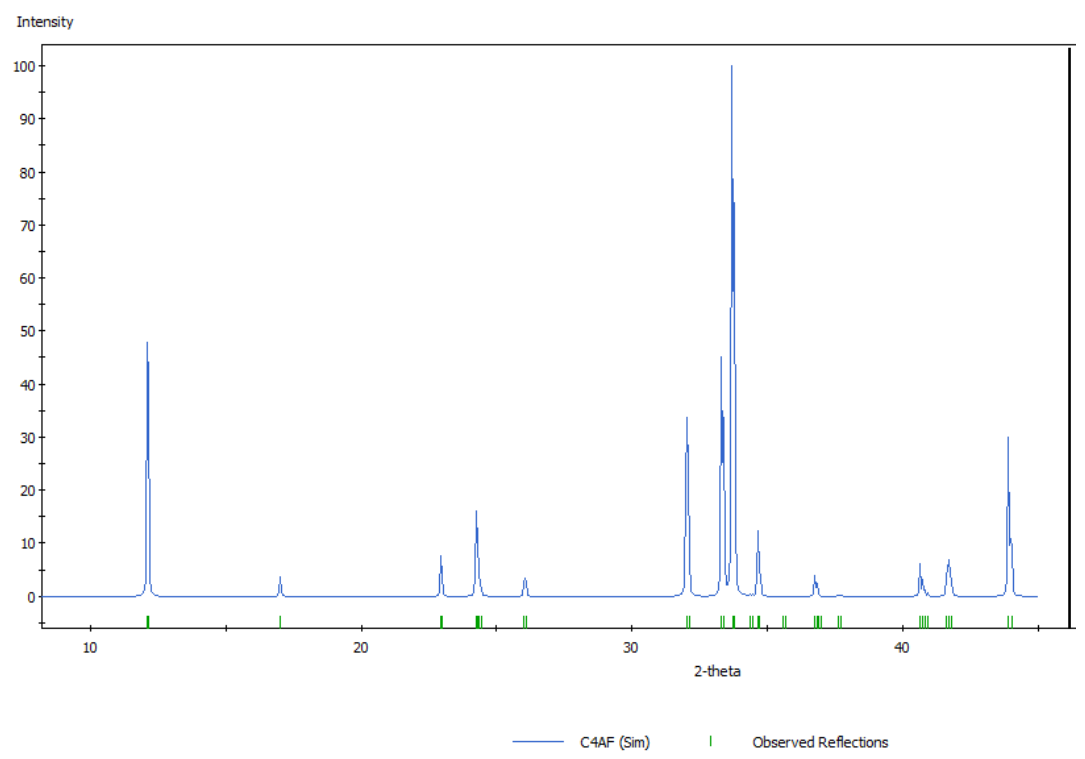

Figure 8: X-ray powder data $(\mathrm{CuK} \alpha)$ diagrams for triclinic of $\mathrm{C}_{4} \mathrm{AF}$ 


\subsection{Hydration of Portland cement}

Hydration is the reaction of non-hydrated cement with water that results in a strong, hard material called paste. It involves a number of reactions which take place simultaneously or one After another. The rate of hydration is affected by a number of parameters such as the composition of the clinker $\left(\mathrm{C}_{3} \mathrm{~S}, \mathrm{C}_{2} \mathrm{~S}, \mathrm{C}_{3} \mathrm{~A}, \mathrm{C}_{4} \mathrm{AF} \ldots\right)$, presence of foreign ions in the clinker, fineness of the cement particles (surface area), w/c ratio, curing temperature and the presence of chemical admixture and supplementary cementitious material (SCM). Due to the complexity of the hydration reaction in Portland cement, it is preferable to discuss the hydration of the individual clinker phase separately.

\subsubsection{Tricalcium silicate (Alite)}

The hydration products of $\mathrm{C}_{3} \mathrm{~S}$ at ambient temperature are an amorphous calcium silicate called C-S$\mathrm{H}$ and calcium hydroxide $\mathrm{Ca}(\mathrm{OH})_{2}$. The hydration of Alite controls the setting and early strength of Portland cement paste. About $70 \%$ of the $\mathrm{C}_{3} \mathrm{~S}$ reacts in 28 days and $90 \%$ will hydrate within one year (Taylor, 1997). The hydration reaction of $\mathrm{C}_{3} \mathrm{~S}$ can be written as:

$$
\begin{gathered}
2 \mathrm{C}_{3} \mathrm{~S}+6 \mathrm{H} \rightarrow \mathrm{C}_{3} \mathrm{~S}_{2} \mathrm{H}_{3}(\mathrm{gel})+3 \mathrm{CH} \\
\begin{array}{c}
\text { Tricalcium } \\
\text { silicate }
\end{array} \\
\text { Water } \quad \mathrm{C}-\mathrm{S}-\mathrm{H} \quad \begin{array}{c}
\text { Calcium } \\
\text { hydroxide }
\end{array}
\end{gathered}
$$

The formula of $\mathrm{C}_{3} \mathrm{~S}_{2} \mathrm{H}_{3}$ is only an approximation because the hydration product varies over a wide range. In addition to the formation of $\mathrm{C}-\mathrm{S}-\mathrm{H}$, calcium hydroxide is also formed because the ratio of $\mathrm{C} / \mathrm{S}$ is lower in $\mathrm{C}-\mathrm{S}-\mathrm{H}$ than in the original $\mathrm{C}_{3} \mathrm{~S}$, which is 3:1 (Mindess et al., 2003).

\subsubsection{Dicalcium silicate (Alite)}

The hydration rate of $\mathrm{C}_{2} \mathrm{~S}$ is lower than $\mathrm{C}_{3} \mathrm{~S}$ because $\mathrm{C}_{2} \mathrm{~S}$ ions are denser than those in $\mathrm{C}_{3} \mathrm{~S}$, which attracts less water for hydration (Odler, 2000). When $\mathrm{C}_{2} \mathrm{~S}$ is mixed with water at room temperature, full hydration takes several years. The hydration reaction of $\mathrm{C}_{2} \mathrm{~S}$ can be written as:

$$
2 \mathrm{C} 2 \mathrm{~S}+4 \mathrm{H} \rightarrow \mathrm{C}_{3} \mathrm{~S}_{2} \mathrm{H}_{3}(\mathrm{gel})+\mathrm{CH}
$$




\section{Dicalcium Water C-S-H Calcium \\ silicate hydroxide}

The hydration product of $\mathrm{C}_{2} \mathrm{~S}$ is almost identical to that of $\mathrm{C}_{3} \mathrm{~S}$, with a smaller amount of calcium hydroxide $(\mathrm{CH})$.

\subsubsection{Tricalcium aluminate $(\mathrm{C} 3 \mathrm{~A})$}

When mixed with water, tricalcium aluminate reacts very quickly to form intermediate phases. The hydration products of $\mathrm{C}_{3} \mathrm{~A}$ are $\mathrm{C}_{2} \mathrm{AH}_{8}$ and $\mathrm{C}_{4} \mathrm{AH}_{19}$ by the following reactions:

$$
\begin{gathered}
2 \mathrm{C} 3 \mathrm{~A}+27 \mathrm{H} \rightarrow \mathrm{C} 2 \mathrm{AH} 8+\mathrm{C} 4 \mathrm{AH} 19 \\
\mathrm{C} 3 \mathrm{~A}+\mathrm{CH}+19 \mathrm{H} \rightarrow \mathrm{C} 4 \mathrm{AH} 19
\end{gathered}
$$

$\mathrm{C}_{2} \mathrm{AH}_{8}$ and $\mathrm{C}_{4} \mathrm{AH}_{19}$ belong to the group called $\mathrm{AFm}$ (alumina, ferric, monosulAFte). Both $\mathrm{C}_{4} \mathrm{AH}_{19}$ and $\mathrm{C}_{2} \mathrm{AH}_{8}$ are thermodynamically unstable and convert to $\mathrm{C}_{3} \mathrm{AH}_{6}$, which belong to the hydrogarnet group.

$$
\mathrm{C} 4 \mathrm{AH} 19+\mathrm{C} 2 \mathrm{AH} 8 \rightarrow 2 \mathrm{C} 3 \mathrm{AH} 6+15 \mathrm{H}
$$

$\mathrm{C}_{4} \mathrm{AH}_{19}$ forms in Portland cement only when the calcium sulfate has been consumed. $\mathrm{C}_{3} \mathrm{~A}$ reacts with the calcium sulfate to form a tricalcium aluminate trisulfate hydrate called Ettringite. After the consumption of calcium sulfate, the Ettringite reacts with the not-yet-consumed tricalcium aluminate to form tricalciuum monosulfate $\left(\mathrm{C}_{3} \mathrm{~A} C \bar{S}_{12} \mathrm{H}\right.$ or $\left.\mathrm{C}_{4} \mathrm{~A} \bar{S} \mathrm{H}_{12}\right)$. The reaction of $\mathrm{C}_{3} \mathrm{~A}$ in the absence of gypsum leads to so-called "flash setting," an undesirable phenomenon that reduces workability and final strength. Gypsum helps regulate $\mathrm{C}_{3} \mathrm{~A}$ hydration to avoid flash setting. The presence of $\mathrm{C}_{3} \mathrm{~A}$ in cement has a negative effect on concrete durability because of the presence of AFm phases, which, when exposed to sulfate solution, can cause sulfate attack (Mindess et al., 2003). In addition, delayed Ettringite (DEF) occurs when concrete is subject to high temperatures $\left(70-88^{\circ} \mathrm{C}\right)$ during the early age of curing (Mindess et al., 2003). High temperature curing destroys the originally formed Ettringite. After cooling, the sulfate is available to form Ettringite again, which can cause expansion in hardened concrete. 


\subsubsection{Aluminoferrite ( $\left.\mathrm{C}_{4} \mathrm{AF}\right)$}

The hydration products of the ferrite phase are similar to those formed during the hydration of $\mathrm{C}_{3} \mathrm{~A}$; they contain $\mathrm{Fe}^{3+}$ as well as $\mathrm{Al}^{3+}$. The hydration reaction is slower than in $\mathrm{C}_{3} \mathrm{~A}$, and it decreases as iron content increases. The hydration reaction of $\mathrm{C}_{4} \mathrm{AF}$ produces less heat in comparison with $\mathrm{C}_{3} \mathrm{~A}$. High $\mathrm{C}_{4} \mathrm{AF}$ content in the cement results in better resistance to sulfate attack (Mindess et al., 2003).

\subsection{Mechanism of hydration}

Different stages can be observed during the hydration of cement. These stages are monitored by heat liberation during hydration:

\subsubsection{Pre-induction period (Stage I)}

As soon as the cement comes in contact with water, rapid dissolution of ionic species into the liquid phase occurs and the first C-S-H forms. The dissolution of alkalis and sulfate present in the cement occurs very quickly, which leads to $\mathrm{K}^{+}, \mathrm{Na}^{+}$and $\mathrm{SO}^{2-}$ ions in the liquid solution. The dissolution of $\mathrm{C}_{3} \mathrm{~S}$ increases the concentration of $\mathrm{Ca}^{2+}$ and $\mathrm{OH}^{-}$in the liquid phase:

$$
\mathrm{C}_{3} \mathrm{~S}+\text { water } \rightarrow 3 \mathrm{Ca}^{2+}+4 \mathrm{OH}^{-}+\mathrm{H}_{2} \mathrm{SO}_{4}{ }^{2-}
$$

$\mathrm{C}_{3} \mathrm{~A}$ dissolves and reacts with $\mathrm{Ca}^{2+}$ and $\mathrm{SO}_{4}{ }^{2-}$ in the liquid phase to form Ettringite. The hydration of $\mathrm{C}_{3} \mathrm{~A}$ in the pre-induction period varies from 5 to $25 \%$ (Lea and Hewlett, 1998). The ferrite phase reacts in a similar manner as $\mathrm{C}_{3} \mathrm{~A}$, leading to the formation of the $\mathrm{AFt}$ phase. The $\beta-\mathrm{C}_{2} \mathrm{~S}$ reacts with water and leads to the formation of C-S-H and the dissolution of $\mathrm{Ca}^{2+}$ and $\mathrm{OH}^{-}$in the liquid, which increase the liquid concentration. Early hydration is slowed due to hydration product being deposited onto the cement grain, forming a barrier between the non-hydrated material and the solution. This barrier causes increased ion concentration due to the dissolved elements in the liquid phase.

\subsubsection{Induction (dormant) period (Stage II)}

The hydration rate in this period slows for few hours, causing the Portland cement concrete to remain plastic during this period. The causes of the induction period have been the subject of many theories, including: 1) the theory that a protective layer is formed around the $\mathrm{C}_{3} \mathrm{~S}$ grains and the induction 
period ends only when this layer is destroyed or becomes more permeable (Jennings, 1986), and 2) that the rate of the reaction in the nucleation period is controlled by the concentration of ions in the solution. When the concentration of $\mathrm{Ca}^{2+}$ and $\mathrm{OH}^{-}$reaches a critical value, hydration products such $\mathrm{CH}$ and C-S-H start to crystallize (nucleation) (Wu and Young, 1984). CH crystals from the solution and C-S-H form on the surface of the grains. The induction period ends when crystal growth begins. Figure 9 shows the different phases of cement hydration: dissolution, nucleation and crystallization of $\mathrm{CH}$ and $\mathrm{C}-\mathrm{S}-\mathrm{H}$

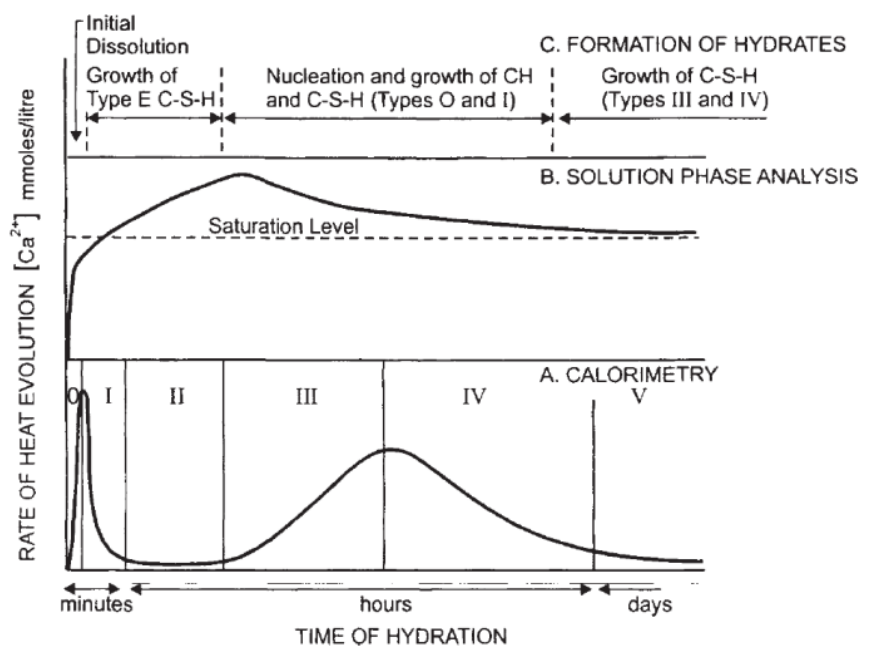

Figure 9: Top figure shows concentration of $\mathrm{Ca}^{2+}$ in solution with time; bottom figure shows different stages of hydration as a function of time (Barnes, 1983)

\subsubsection{Acceleration period (Stage III)}

This phase occurs in the first 3-12 hours After mixing. An increase in the rate of hydration occurs in this phase, with accelerated dissolution of calcium silicate and aluminate as a consequence increased liberated heat. The crystal $\mathrm{CH}$ (Portlandite) precipitates from the liquid phase with a decreased concentration of $\mathrm{Ca}^{2+}$ and $\mathrm{OH}^{-}$in the pore solution. The calcium sulfate continues to dissolve in the liquid and $\mathrm{SO}_{4}{ }^{2-}$ concentration decreases due to the formation of Ettringite, and also due to adsorption of $\mathrm{SiO}_{4}{ }^{2-}$ for the formation of C-S-H (Lea and Hewlett, 1998). 


\subsubsection{Post-acceleration stage (Stage IV)}

In this phase, the rate of hydration decreases slowly and hydration becomes diffusion-controlled. C$\mathrm{S}-\mathrm{H}$ continues to form from $\mathrm{C}_{3} \mathrm{~S}$ and $\mathrm{C}_{2} \mathrm{~S}$, with the contribution of $\mathrm{C}_{2} \mathrm{~S}$ becoming more pronounced with time. When the calcium sulfate is consumed, $\mathrm{SiO}_{4}{ }^{2-}$ concentration is reduced. The AFt phase reacts with additional $\mathrm{C}_{3} \mathrm{~A}$ and $\mathrm{C}_{2}(\mathrm{~A}, \mathrm{~F})$ to form monosulfate aluminate. With a high w/c ratio, the hydration process continues until all the anhydrate cement is consumed. Larger cement particles may take longer to hydrate. At low w/c, the hydration reaction may stop, even with the availability of significant amounts of non-reacted cement.

\subsection{Multiscale level of cement paste}

Predicting properties of cement paste or concrete is difficult due to the composite behavior, heterogeneity of the material and the existence of multi-scale length. An understanding of multi-scale levels of cement paste and the interactions between these scales is fundamental to understanding the properties of concrete. Concrete scale can be divided in three levels: macro, micro and nano, as illustrated in Figure 10.

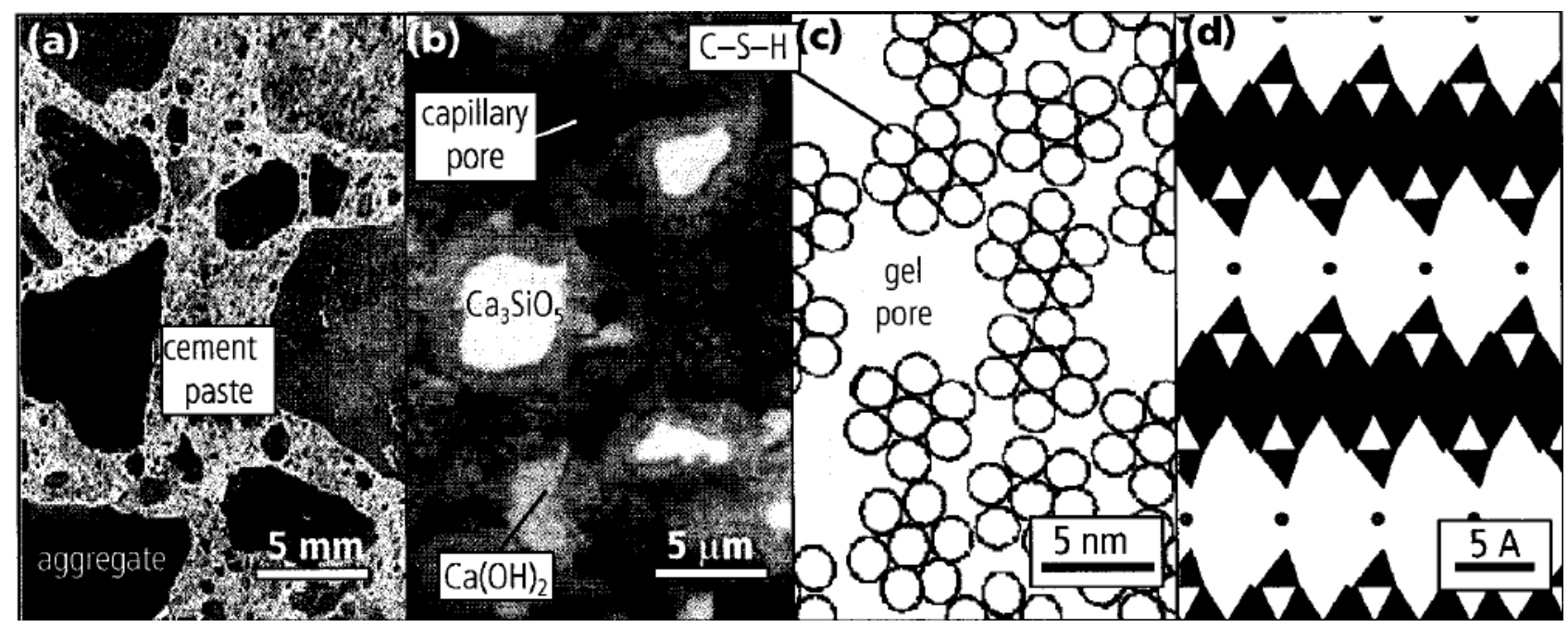

Figure 10: Structural hierarchy in concrete

(a) Optical image of concrete showing aggregates in a matrix of hardened cement paste, (b) Back scattered electron image of hardened cement paste showing unhydrated $\mathrm{Ca}_{3} \mathrm{SiO}_{5}$ (brightest phase), 
$\mathrm{Ca}(\mathrm{OH}) 2$ (dark gray), $\mathrm{C}-\mathrm{S}-\mathrm{H}$ (light gray), and apillary pores (black), (c) Agglomerated particles of C-S-H creating a gel-like structure of high surface area, (d) Layered crystal structure of 1.4-nm tobermorite, a natural mineral whose structure is related to that of $\mathrm{C}-\mathrm{S}-\mathrm{H}$ at the sub-particle level.

\subsubsection{Macro scale}

The term macro-scale refers to gross microstructure visible to the human eye $(1 / 5 \mathrm{~mm})$ (Mehta and Monteiro, 2014). At macro-scale level, structures have two phases: a composite of aggregate particles of different sizes and shapes and the binding element composed of hydrated cement paste. Macro pores (entrapped air) can also be seen, having been caused due to a lack of vibration, which has a negative effect on the mechanical properties of the paste.

\subsubsection{Micro structure scale}

The microstructure is the $10^{6}$ magnified portion of the macrostructure. At the microscopic level, the two phases are not homogenously distributed and each scale represents a new composite. For example, the hydrated cement appears to be dense in some areas and porous in others. In addition, the hydrated cement paste appears to be different close to the aggregate zone (ITZ: interfacial transition zone). C-S-H at micro-scale depends on many factors including the nature of the cement, type, admixture, temperature, period of hydration and w/c ratio. Study of the microstructure includes morphology (sizes and shapes), surface area, density, porosity, pore shape and pore distribution (Ramachandran, 1984). Many parameters at the microstructure level are connected to each other; no individual parameter can control the properties of the paste. The morphology of C-S-H at micro-scale level has been described as foils and flakes, crumpled sheets and rods, and tube for AFt, depending of the degree of hydration (Ramachandran, 1984). At a later age, two types of products in C-S-H can be distinguished: the "inner product" formed in the boundaries of the original anhydrous grains and the "outer product" filling the space originally filled with water. Diamond (2004) found that the ratio of $\mathrm{Ca} / \mathrm{Si}$ is higher in the inner products than in the outer ones. 
In addition to the solid phase, the hydrated cement paste contains capillary voids, which are the empty spaces that occur when the hydration product takes the space of water and cement. Capillary voids range from 3-5 um at early ages to 10-50 $\mathrm{nm}$ at late ages (Mehta and Monteiro, 2001). As cement hydration occurs, pore volume decreases, and the channels connecting them are broken. Also, the higher the w/c, the higher the pore volume.

At this scale, calcium hydroxide $(\mathrm{CH})$ can be distinguished from $\mathrm{C}-\mathrm{S}-\mathrm{H}$ because it has a different size and shape. The morphology of $\mathrm{CH}$ is affected by available growth space, temperature and impurities in the cement (Mehta and Monteiro, 2001).

\subsubsection{Nano scale level}

At the nano-scale level of less than $10^{-9} \mathrm{~m}$, a number of methods are used to study the structure of CS-H: nuclear magnetic resonance (NMR), scanning electron microscope (SEM), transmission electron microscopy (TEM), atomic force microscopy (AFM), small-angle scattering (SANS) and small-angle X-ray scattering (SAXS). These techniques cannot solve the structure of the cement paste, but they can provide some characteristics of $\mathrm{C}-\mathrm{S}-\mathrm{H}$ :

\subsubsection{Ca/Si ratio}

Stoichiometry is the quantitative relationship between the reactants and products in a chemical reaction. The stoichiometry of $\mathrm{C}-\mathrm{S}-\mathrm{H}$ is not well defined and the dashed line between $\mathrm{C}, \mathrm{S}$, and $\mathrm{H}$ is to say that C- S-H compositions are known but the stoichiometry of C-S-H is not. To differentiate between different compositions, the ratio $(\mathrm{Ca} / \mathrm{Si})$ used can vary depending on cement composition (Taylor, 1997). In addition, the $\mathrm{Ca} / \mathrm{Si}$ ratio varies irregularly from one point to another in the same cement paste sample, generally fluctuating between 1.5 and 2.3 with a mean of 1.75 , but the value can be higher than 2.3 and lower than 1.2. Figure 11 shows the frequency of $\mathrm{Ca} / \mathrm{Si}$ ratio over a 2.5 year duration. 


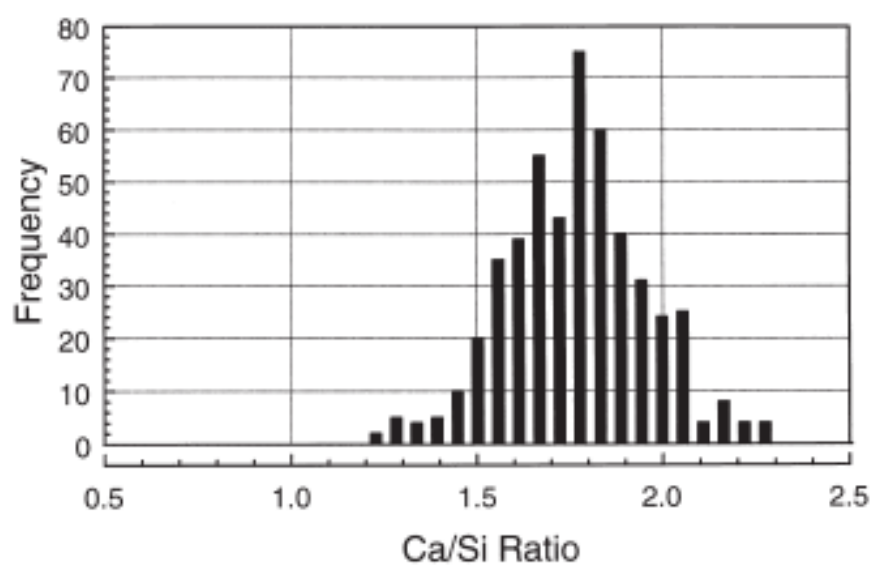

Figure 11: $\mathrm{Ca} / \mathrm{Si}$ ratio frequency histogram for $\mathrm{C}-\mathrm{S}-\mathrm{H}$ in Portland cement paste aged 1 day to 3 years (TEM microanalysis) (Richardson, 1999)

\subsubsection{Water content}

Water exists in hydrated cement paste in many forms. The best classification of different types of water is by Sierra (Gmira, 2003), which is based on structural and energy considerations. However, the dividing line between types is not always distinguishable. The different types are:

a) Interlayer water, the water between the layers of C-S-H, which is suggested to be a monolayer held by hydrogen bonding (Mehta and Monteiro, 2014). The C-S-H shrinks considerably when interlayer water is lost.

b) Adsorbed water is the water held on the surface of the hydrated pores by intermolecular forces, which decrease as the distance from the surface increases.

c) Capillary water is the water present in voids larger than 50A (Mehta and Monteiro, 2014).

\subsubsection{Layered structure}

$\mathrm{C}-\mathrm{S}-\mathrm{H}$ has a layer structure that resembles Tobermorite and Jennite. The major difference between $\mathrm{C}-\mathrm{S}-\mathrm{H}$ and these minerals is the silicate chain. $\mathrm{C}-\mathrm{S}-\mathrm{H}$ has dimeric, pentameric and higher chains of $\mathrm{SiO}_{4}$ in a sequence of 2, 5, 8, (3n-1). In the NMR study, $\mathrm{SiO}_{4}$ represents one unit of the silicate chain, and these units can be connected in different forms of $\mathrm{Q}^{\mathrm{n}}$ where $\mathrm{Q}^{\mathrm{n}}$ represents the connectivity: 
$\mathrm{Q}^{0}$ : isolated tetrahedral

$\mathrm{Q}^{1}$ : end tetrahedrals

$\mathrm{Q}^{2}$ : middle tetrahedral

During cement hydration, the $\mathrm{Q}^{0}$ starts to decrease, accompanied by the formation of $\mathrm{Q}^{1}$ and $\mathrm{Q}^{2}$. The formation of $\mathrm{Q}^{3}$ and $\mathrm{Q}^{4}$ was not observed (Taylor, 1997). Figure 12 shows the different types of connectivity between the tetrahedral, from single tetrahedral $\left(Q^{1}\right)$ to three units of tetrahedrals $\left(Q^{2}\right)$.

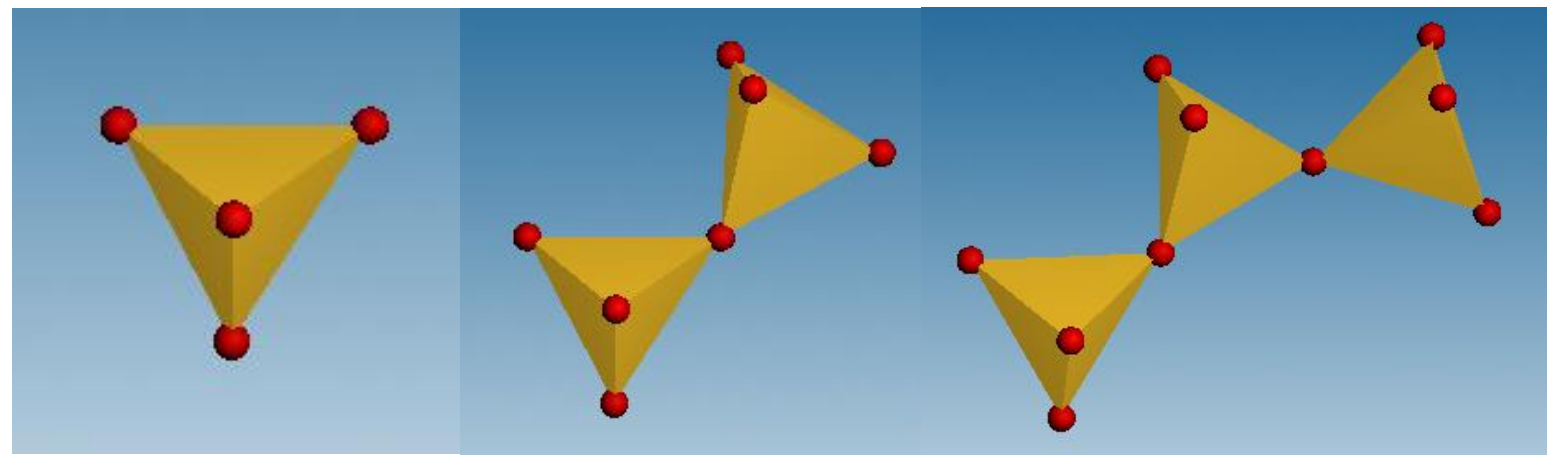

$$
\left.\left.\mathrm{Q}^{0} \text { (one unit } \mathrm{SiO}_{4}\right) \quad \mathrm{Q}^{1} \text { (two units } \mathrm{SiO}_{4}\right) \quad \mathrm{Q}^{2} \text { (three unit } \mathrm{SiO}_{4} \text { ) }
$$

Figure 12: Different connections between tetrahedral $\mathrm{SiO}_{4}$

During the first 24 hours of cement hydration, the $\mathrm{Q}^{0}$ (monomer) starts to decrease, accompanied by the formation of $\mathrm{Q}^{1}$ (dimer). At later ages, longer linear chains of species are formed; mainly pentamer and octamer following the $3 \mathrm{n}-1$ rule. Dimer represents $40 \%$ of the total chain, even After six months of hydration. Figure 13 shows the distribution of ion species over time.

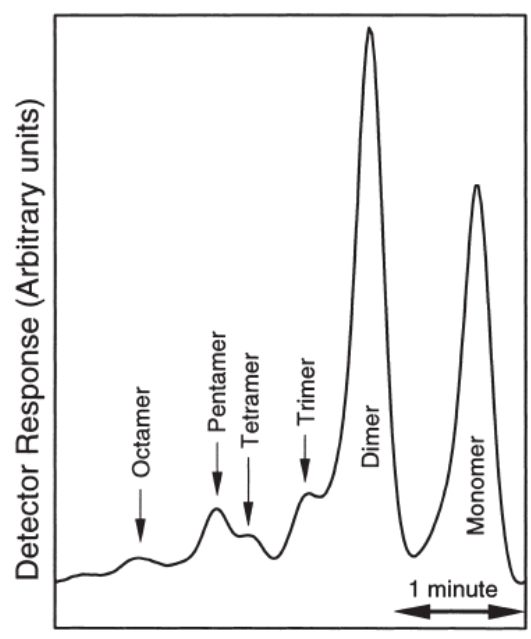

Time 
Figure 13: TMS-GPC chromatogram of white Portland cement paste hydrated for 3 months at $20^{\circ} \mathrm{C}$;

$$
\mathrm{W} / \mathrm{C}=0.55
$$

The mean silicate chain is the number of silicate tetrahedrals in a chain, calculated based on the ratio of $\mathrm{Q}^{2} / \mathrm{Q}^{1}$

$$
\text { Mean } \text { chain }=2\left(1+\frac{Q^{2}}{Q^{1}}\right)
$$

Figure 14 shows the relationship between the mean silicate chain and $\mathrm{Ca} / \mathrm{Si}$. It clearly shows that the mean silicate chain decreases as $\mathrm{Ca} / \mathrm{Si}$ ratio increases (Lagerblad et al., 1999).

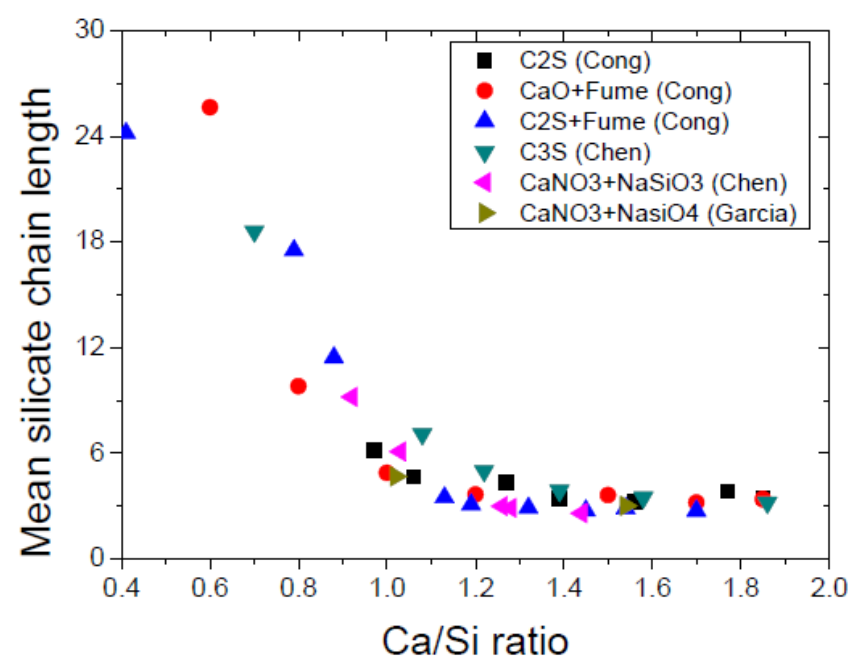

Figure 14: Mean silicate chain relationship with $\mathrm{Ca} / \mathrm{Si}$ ratio (Hou, 2014)

\subsubsection{Mechanical properties}

Nano-indentation is an experimental tool used to predict the mechanical properties of cement paste at nano- and micro-scale levels to show mechanical properties such as elastic modulus and hardness. Nano-indentation consists of pushing an indenter tip of a known geometry into the surface of any material. Load and penetration of the indenter are recorded and plotted on a graph, and indentation modulus (M) and hardness $(\mathrm{H})$ are calculated. The applied load is in the micro-Newton range. Figure 14 shows that indentation modulus $(\mathrm{M})$ is proportional to packing fraction. As packing increases along with indentation modulus $(\mathrm{M})$, the agreement of the results between nano-indentation and 
atomistic simulation increases as well. The relationship between Young modulus and the indentation modulus (M), bulk, shear modulus and Poisson ratio is provided by this equation:

$$
M=\frac{E}{1-v^{2}}=4 G \frac{3 K+G}{3 K+4 G}
$$

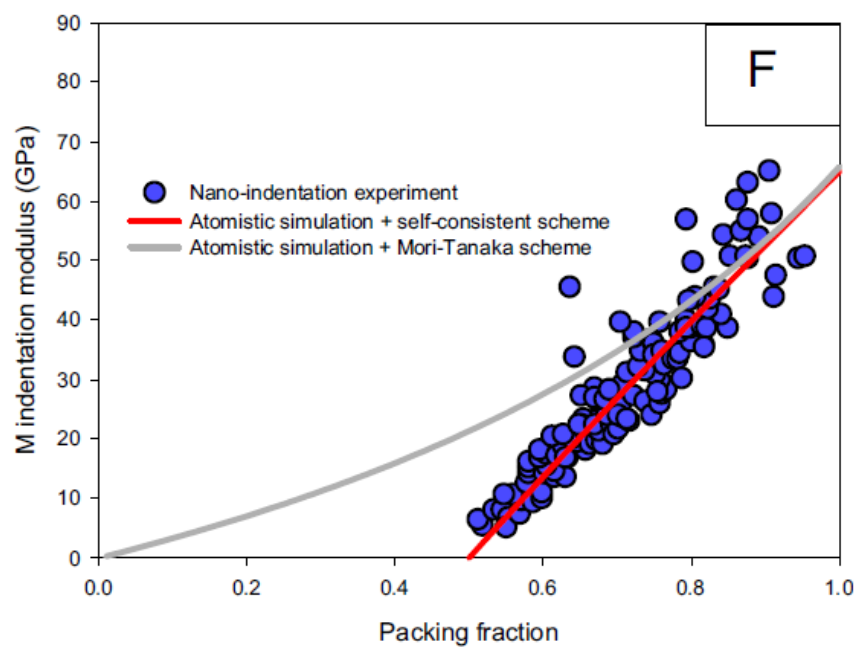

Figure 15: Relationship between packing density and indentation modulus (Pellenq et al., 2009)

Young modulus is calculated from the indentation modulus, which is calculated from experiments. In regards to nano-indentation method, Canstantinides et al. (2013) found that there are two types of C-S-H: low density (LD) and high density (HD), with a packing factor of 0.64 and 0.74 , respectively. The mean Young modulus of C-S-H is estimated to range between 20-30 GPa for LD and HD, respectively.

\subsection{Mineral Analogy of C-S-H (ordered structure)}

There are at least thirty crystalline structures that show similar composition to C-S-H. However, although the chemical compositions are similar, these minerals differ in their atomic arrangement, $\mathrm{Ca} / \mathrm{Si}$ ratio, and the number of $\mathrm{OH}$ and $\mathrm{H}_{2} \mathrm{O}$. Most high-resolution TEM and extensive X-rays show that C-S-H has the structure of Tobermorite and Jennite (Taylor, 1997). 


\subsubsection{Tobermorite $14 \AA$}

Tobermorite $14 \AA\left(\mathrm{Ca}_{5} \mathrm{Si}_{6} \mathrm{O}_{26} \mathrm{H}_{18}\right)$ has $\mathrm{SiO}_{4}$ tetrahedrals in a linear chain, twisted so they repeat at three tetrahedrals. This type of arrangement is called dreierkette. Tobermorite has a layer structure consisting of a central part of $\mathrm{CaO}_{2}$ in which all the oxygen atoms are shared with the $\mathrm{SiO}_{4}$ tetrahedrals. Only two tetrahedral share their oxygen with $\mathrm{CaO}_{2}$; these are called paired tetrahedrals. The third is a bridging tetrahedral, which is positioned between the two paired tetrahedrals. The interlayer space between the individual layers is filled with $\mathrm{Ca}^{2+}$ and water molecules. Tobermorite is present in three different types, depending on water content: Tobermorite $14 \AA, 11 \AA$ and $9 \AA$ (Lea and Hewlett, 1998). Studies show that Tobermorite $14 \AA$ transforms into Tobermorite $11 \mathrm{~A}$ when heated to $80-100^{\circ} \mathrm{C}$, and additional heating to $300^{\circ} \mathrm{C}$ transforms $11 \AA$ to $9 \AA$ (Lea and Hewlett, 1998). Tobermorite $11 \AA$ is present in two model structures: the Hamid structure has independent layers (Hamid, 1981), while the Merlino structure has chemically bonded layers (Merlino et al., (2001)). Figures 16, 17 and 18 show the structure of Tobermorite $14 \AA$ with details of pair and bridging tetrahedrals, interlayers filled with water and calcium, and the $\mathrm{CaO}_{2}$ layer between the silicate chains. The X-ray diffraction of Tobermorite $14 \AA$ is shown in Figure 20 .

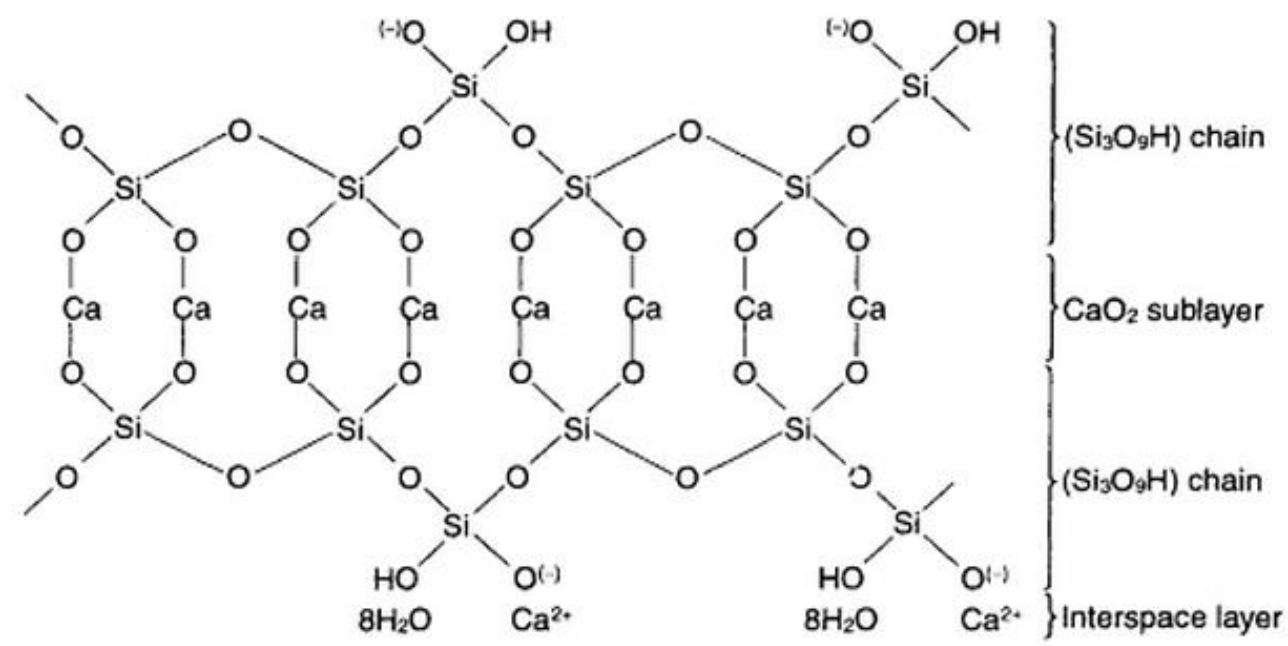

Figure 16: Idealized chemical structure of Tobermorite 14A (Hewlett, 2004) 


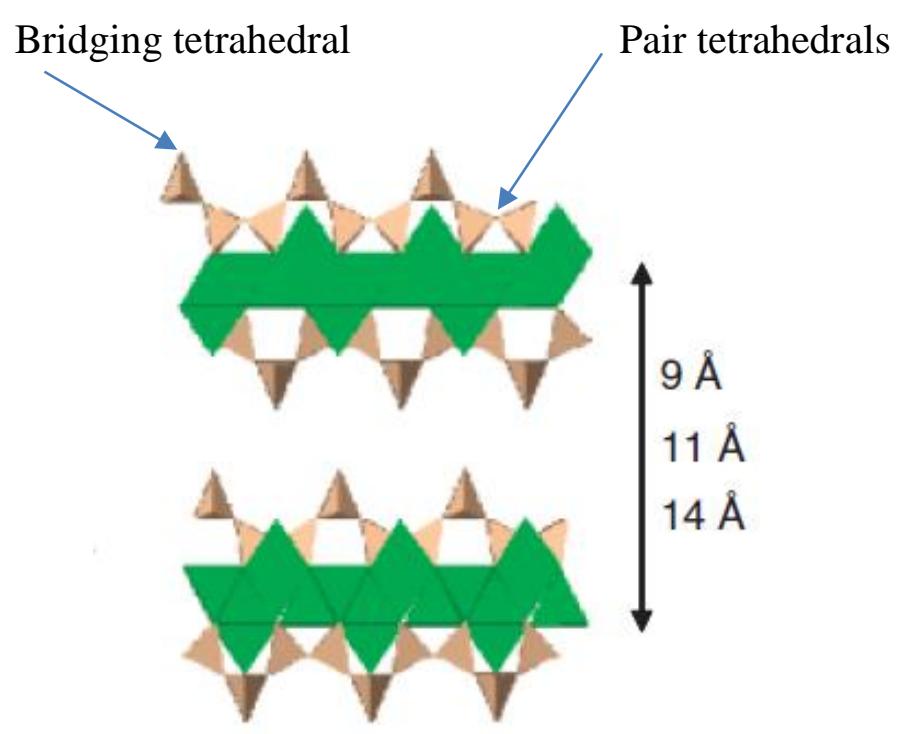

Figure 17: View of bridging tetrahedral and Pair tetrahedrals of Tobermorite (Shahsavari, Buehler, Pellenq, \& Ulm, 2009)

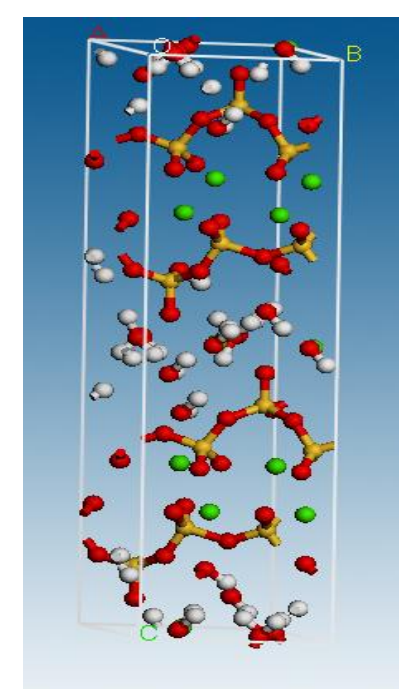

Figure 18: Unit cell of Tobermorite 14A where $\mathrm{Ca}$ is presented in green, silicon in orange, oxygen in red, hydrogen in white, and the white line represents the unit cell 


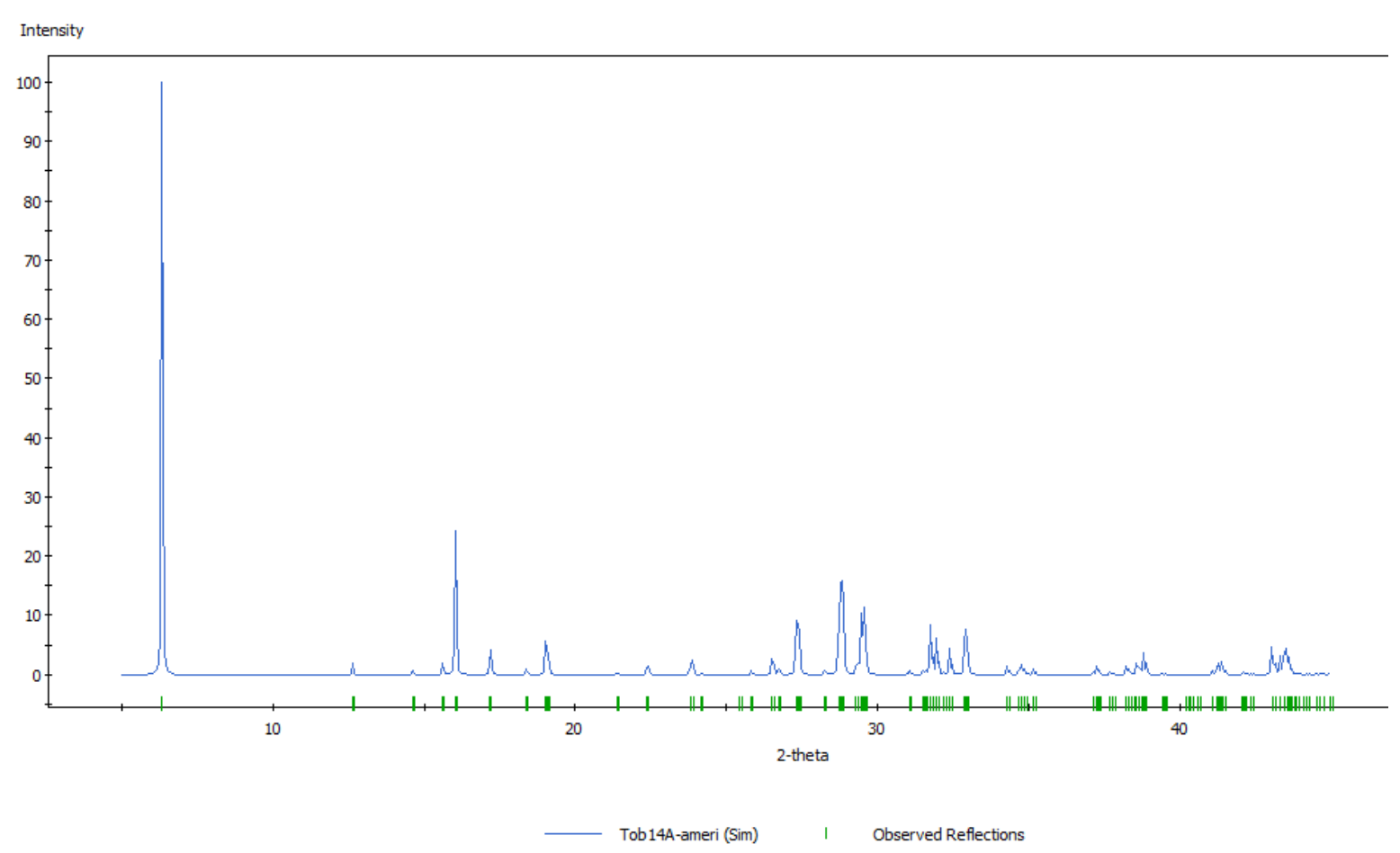

Figure 19: X-ray diffraction of Tobermorite 14A

\subsubsection{Tobermorite $11 \AA$}

Two types of Tobermorite $11 \AA$ crystal structure have been determined. Hamid (1981) and Merlino et al. (2001). According to Hamid, the Tobermorite structure in Figure 20 has a silicate chain in one layer independent of the other. However, in the Merlino structure presented in Figure 21, the silicate chain is connected and attached to the Ca-O layer. The Hamid structure comes in three forms that depend on the $\mathrm{C} / \mathrm{S}$ ratio:
$\mathrm{C} / \mathrm{S}=0.667$
$\mathrm{Ca}_{4}\left[\mathrm{Si}_{6} \mathrm{O}_{14}(\mathrm{OH})_{4}\right] \cdot 2 \mathrm{H}_{2} \mathrm{O}$
$\mathrm{C} / \mathrm{S}=0.830$
$\mathrm{Ca}_{5}\left[\mathrm{Si}_{6} \mathrm{O}_{16}(\mathrm{OH})_{4}\right] \cdot 2 \mathrm{H}_{2} \mathrm{O}$
$\mathrm{C} / \mathrm{S}=1$

$$
\mathrm{Ca}_{6}\left[\mathrm{Si}_{6} \mathrm{O}_{18}\right] \cdot 2 \mathrm{H}_{2} \mathrm{O}
$$
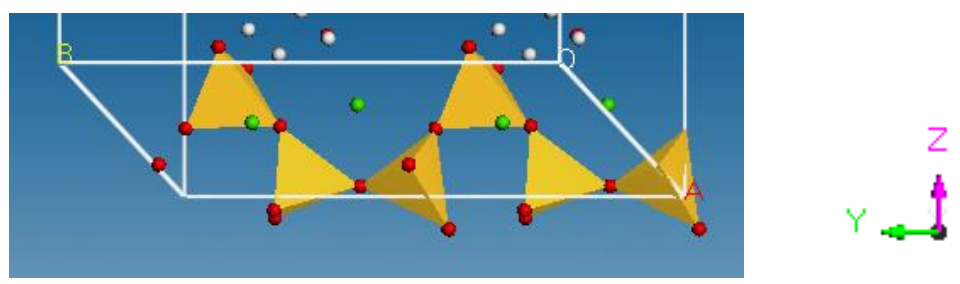

Figure 20: Hamid crystal structure showing linear silicate 


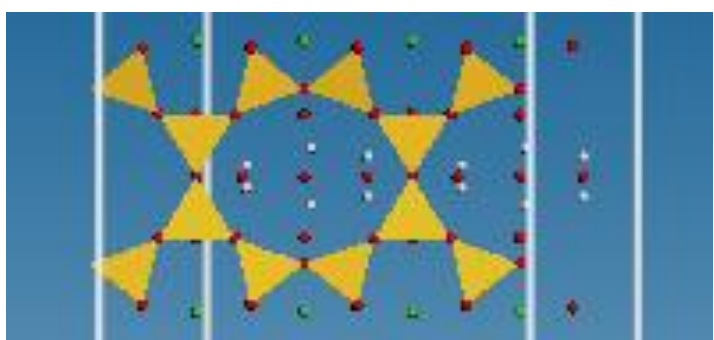

Figure 21: Merlino crystal structure showing silicate tetrahedral connected at the interlayer

\subsubsection{Jennite}

Jennite $\mathrm{Ca}_{9}\left(\mathrm{Si}_{6} \mathrm{O}_{18} \mathrm{H}_{2}\right)(\mathrm{OH})_{6} 8 \mathrm{H}_{2} \mathrm{O}$ is a natural mineral with a linear layer structure composed of $\mathrm{SiO}_{4}$ tetrahedrals that are twisted so they repeat at intervals of three tetrahedrals. This arrangement is called dreierkette. The $\mathrm{Ca} / \mathrm{Si}$ ratio of Jennite is 1.5 times higher than Tobermorite, and the layer has a thickness of $1.05 \mathrm{~nm}$. The structure of Jennite has many similarities to Tobermorite, with a difference in the presence of the $\mathrm{OH}$ group in the sublayer $\mathrm{CaO}-\mathrm{OH}$ and the absence of $\mathrm{SiOH}$ at the terminal. When Jennite is heated to $70-90^{\circ} \mathrm{C}$, the water molecules are lost and the unit cell shrinks in $\mathrm{C}$ direction (Bonaccorsi et al., 2004). The unit cell of Jennite and its X-ray diffraction is shown in Figures 22 and 23 , respectively.

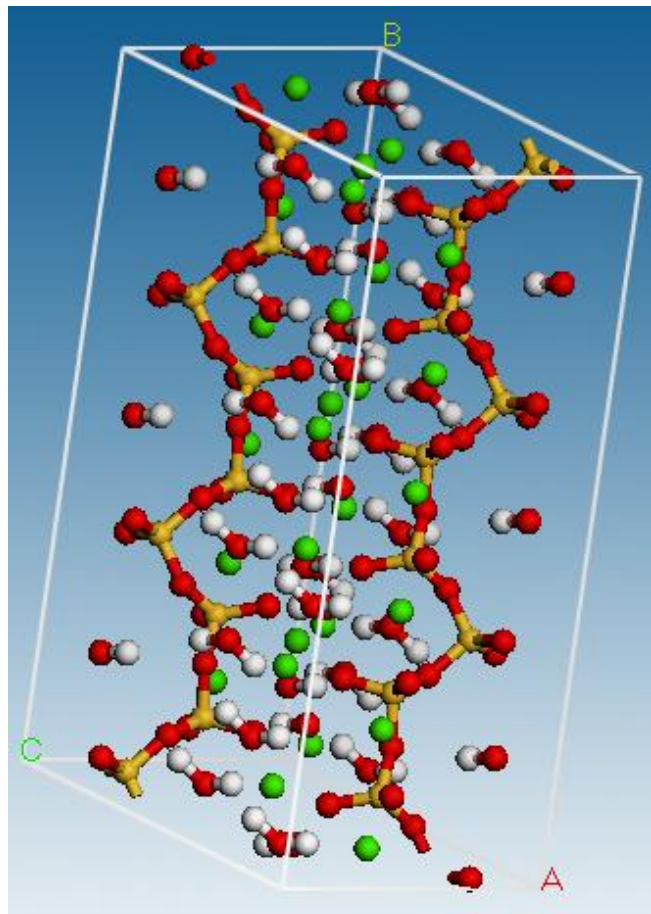

Figure 22: Unit cell of Jennite where $\mathrm{Ca}$ is presented in green, silicon in orange, oxygen in red, hydrogen in white, and the white line represents the unit cell 


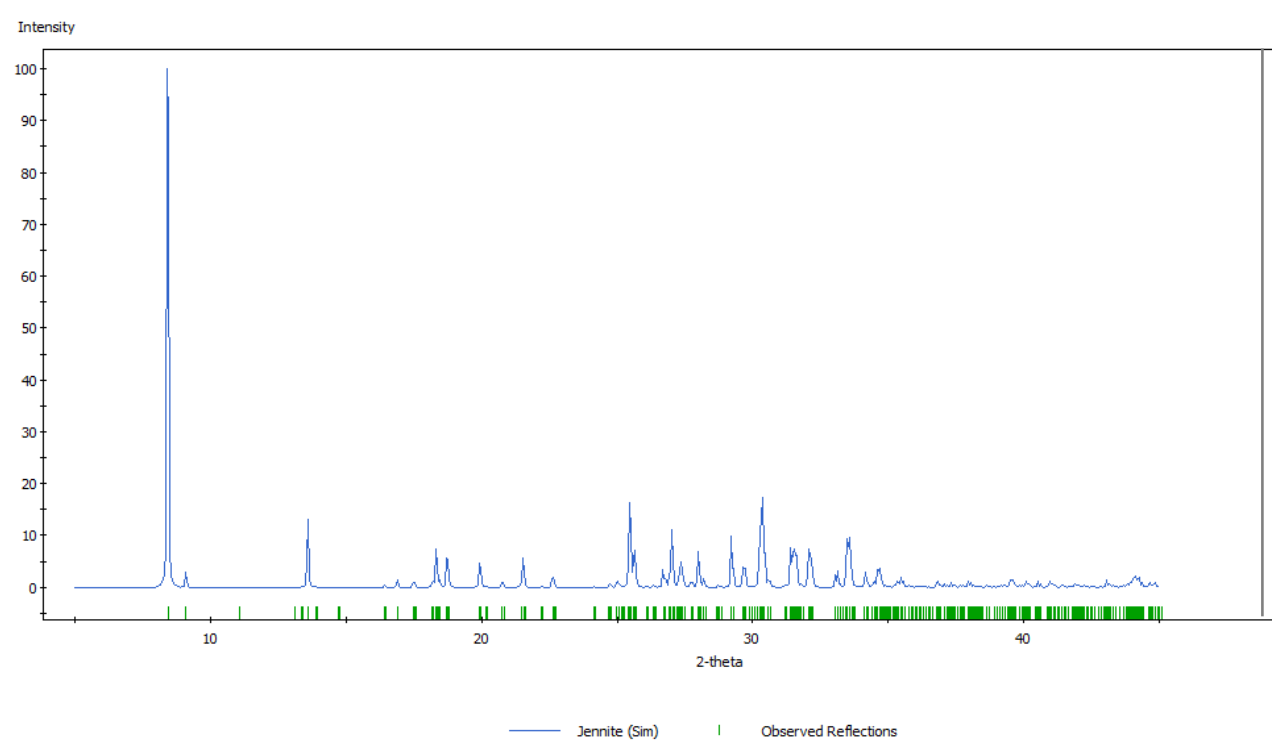

Figure 23: X-ray diffraction of Jennite

\subsubsection{C-S-H (I and II)}

The purpose of producing C-S-H (I) is to synthesize Tobermorite 14A. C-S-H (I) is the reaction product between calcium hydroxide and silica or between sodium silicate and calcium salts (Lea and Hewlett, 1998). C-S-H (I) has a structure similar to Tobermorite, with some missing bridging silicate tetrahedrals that follow the $3 \mathrm{n}-1$ chain rule. $\mathrm{C}-\mathrm{S}-\mathrm{H}$ (I) has a $\mathrm{Ca} / \mathrm{Si}$ molar ratio between $0.8-1.5$ and a reduction of the mean silicate chain. On the other hand, C-S-H (II) is produced by hydrating $\mathrm{C}_{3} \mathrm{~S}$ or $\beta-\mathrm{C} 2 \mathrm{~S}$ in excess water and repeatedly removing the liquid and replacing it with pure water until the $\mathrm{Ca} / \mathrm{Si}$ ratio reaches the value of 2 . Using electron diffraction, the $\mathrm{C}-\mathrm{S}-\mathrm{H}$ (II) shows a similar structure to Jennite with some disorder and finite chain length (Taylor, 1997).

\subsection{Model of C-S-H at nano scale}

To predict the performance and properties of concrete, it is important to have a model that explains the behavior of C-S-H under different conditions. The best-known models are discussed below:

\subsubsection{Power and Brunauer (1964)}

In the Power model, C-S-H is composed of particles held together by Van der Waals forces. Although the van der Waals interactions are weak, they can provide high concrete strength due to high surface 
area $\left(200 \mathrm{~m}^{2} / \mathrm{g}\right)$. In addition, the model shows a fraction of chemical bonding (cross link) between the gel particles. The water in C-S-H can be categorized into three types:

a) Water combined chemically in the hydration product, which can only be evaporated at $1000^{\circ} \mathrm{C}$.

b) Adsorbed water, which is the water held on the surface of the gel particles by physical forces (interlayer water).

c) The capillary water, which is free water inside the pores (Popovics, 1998).

The swelling in the Power model is caused by the presence of colloidal Ettringite, and the creep is caused by water squeezing between the particles, as shown in Figure 24.
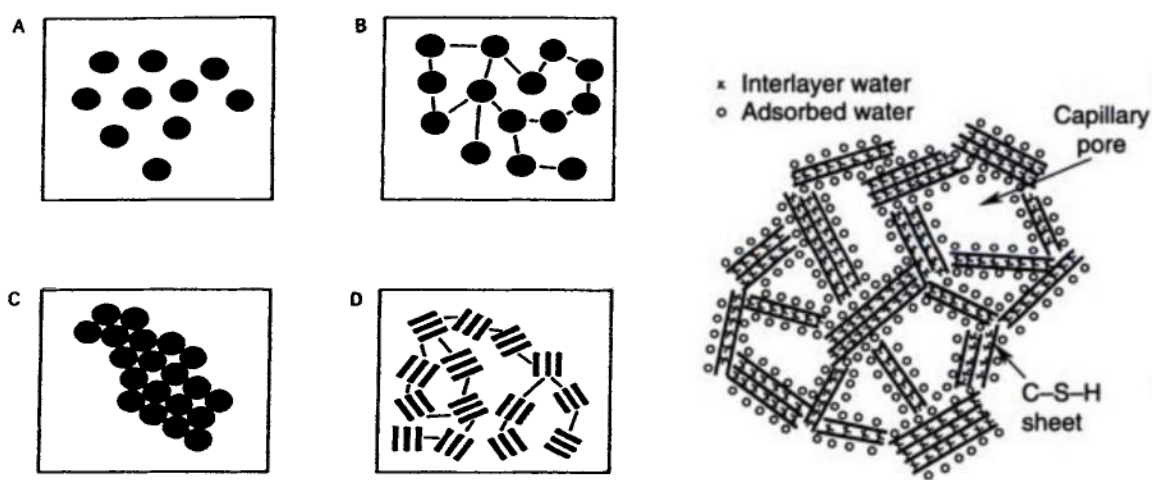

Figure 24: Schematic representation of Powers model (Feldman and Sereda, 1968)

\subsubsection{Feldman-Sereda model}

The Feldman-Sereda model considers cement paste to be poorly crystalline layers of silicate, with the water having a more complex role than in the Power-Brunauer model. The water in the interlayer has a structural role, which is to connect two layers together and contribute to the rigidity of the system. The interlayer water can enter or leave the system (Feldman and Sereda, 1968). Figure 25 represents the Feldman-Sereda shematic model (Feldman and Sereda, 1968). 


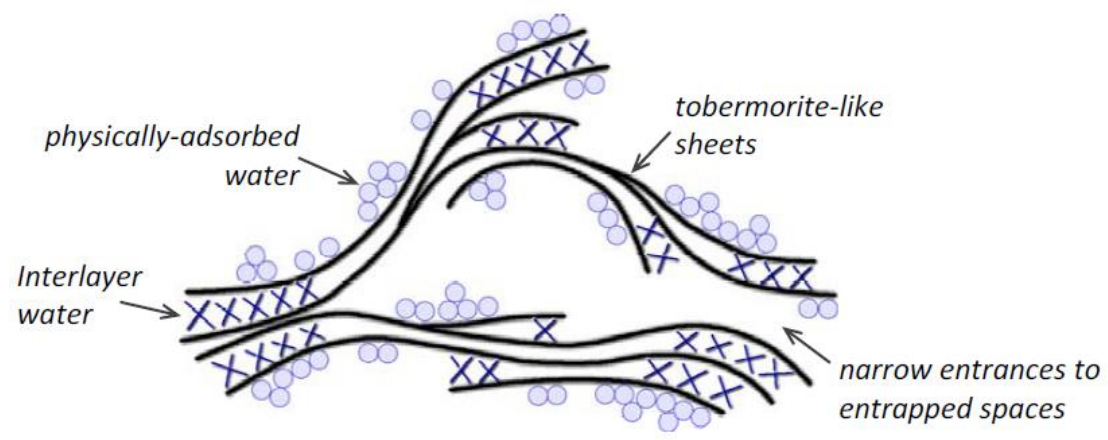

Figure 25: Schematic representation of Feldman-Sereda model (Feldman and Sereda, 1968)

\subsubsection{Jennings model}

Jennings proposed a colloidal model to explain the discrepancy between the results of investigations of the surface area and pore size distribution. The discrepancy does not only occur from one sample to another; results are different in the same sample. The model shown in Figure 27 is composed of basic blocks of $2.2 \mathrm{~nm}$ with equated shape; however, for simplicity, the block is shown as spherical. These blocks cluster to form units called globules, which are $5.6 \mathrm{~nm}$ in size. Jennings proposed two types of C-S-H: HD (High Density) C-S-H, where the structure is very compacted and $\mathrm{N}_{2}$ (adsorption measurement) cannot penetrate, and LD C-S-H (Low Density), with loosely packed globules that are accessible by $\mathrm{N}_{2}$. These two types of C-S-H have a gel porosity of $37 \%$ and $24 \%$ for LD and HD respectively, which can help explain the difference in surface area (Jennings, 2000). In addition, the pore sizes within these globules are similar for HD and LD and are not dependent on the volume of HD and LD from one cement to another (Jennings, 2000). 

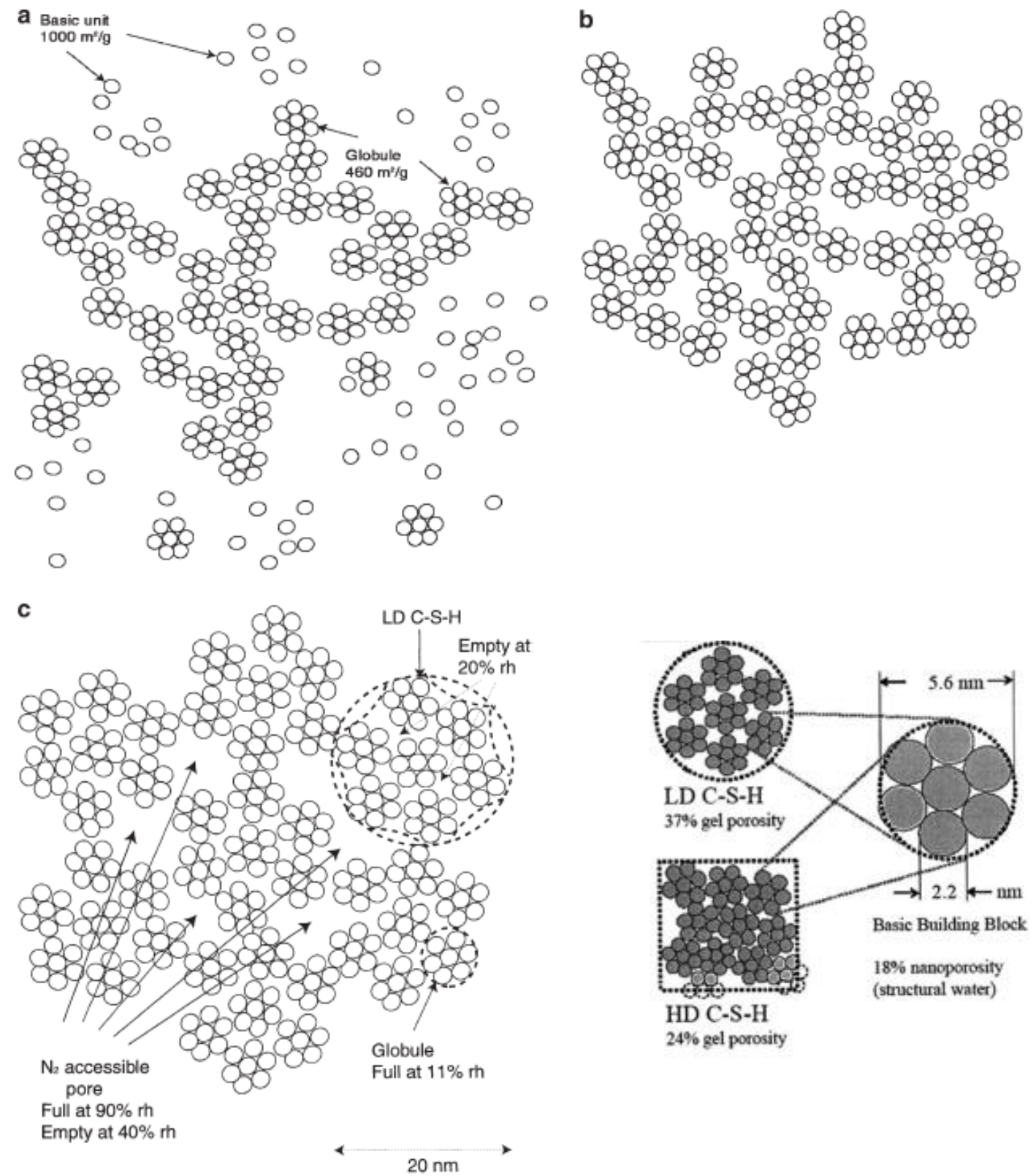

Figure 26: a) Basic building block, b) Globules, c) LD C-S-H. d) HD C-S-H and accessibility of $\mathrm{N}_{2}$ to pores (Jennings, 2000)

\subsubsection{Atomic model}

In 1956, Taylor determined a relationship between the structures of C-S-H and Tobermorite made up of a central part with calcium hydroxide with missing hydrogen atoms. This sheet of $\mathrm{CaO}_{2}$ is located between two parallel rows of silicate chains. These chains are twisted in a plan perpendicular to the $\mathrm{CaO}_{2}$ sheet. Two thirds of the tetrahedrals are connected to the central chain of $\mathrm{CaO}_{2}$, sharing oxygen atoms. The remaining third not connected to the central sheet is called a "bridging" tetrahedral. In 
addition, between these layers, there is calcium and water molecule called the interlayer. Tobermorite $\mathrm{Ca}\left(\mathrm{Si}_{6} \mathrm{O}_{18} \mathrm{H}_{2}\right) \cdot \mathrm{Ca}_{4} \mathrm{H}_{2} \mathrm{O}$ has a $\mathrm{C} / \mathrm{S}$ ratio of $0.83(5 / 6)$; in contrast $\mathrm{C}-\mathrm{S}-\mathrm{H}$ has an average $\mathrm{C} / \mathrm{S}$ of 1.7. To increase the $\mathrm{C} / \mathrm{S}$ ratio, Taylor proposed omitting the bridging tetrahedrals not directly connected to the central sheet $\mathrm{CaO}_{2}$. Because the oxygen atoms are shared with the adjacent tetrahedral, the removal of the bridge leads only to the removal of $\mathrm{SiO}_{2}$ and the transfer of the hydrogen atom to other position in the structure. In addition, the removed hydrogen atoms can be replaced by $\mathrm{Ca}$ atoms in the interlayer, as shown in Figure 27.

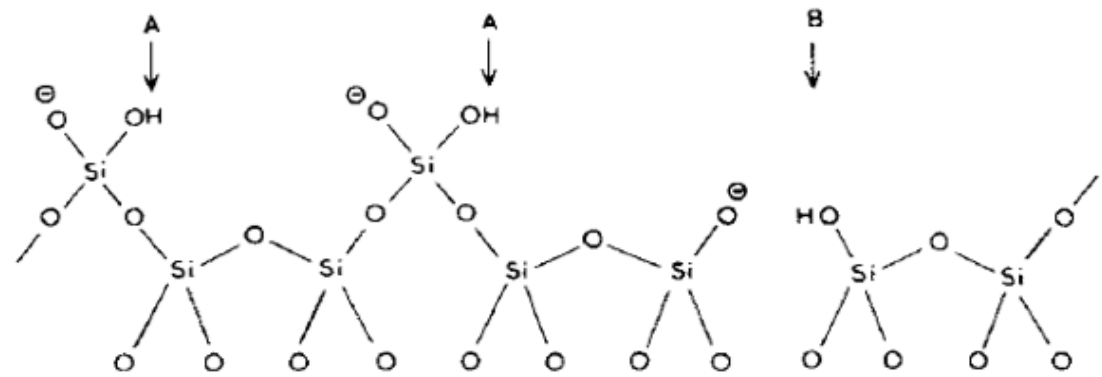

Figure 27: Silicate chain of Tobermorite showing the probable position of $\mathrm{H}$ atoms and omission of bridging tetrahedrals (Taylor, 1986)

Thirty years later, Taylor proposed a C-S-H model that is a mixture of Tobermorite $14 \AA$ and Jennite structures to explain the high value of $\mathrm{C} / \mathrm{S}$ ratio in the cement paste (1.7 to 2) as dimeric $1.4 \mathrm{~nm}$ Tobermorite and Jennite, with C/S ratios of 1.25 and 2.25 respectively. In both Tobermorite and Jennite, the silicate chain is considered infinite and the extreme $\mathrm{C} / \mathrm{S}$ ratio of Jennite $\mathrm{Ca}_{9}\left(\mathrm{Si}_{6} \mathrm{O}_{18} \mathrm{H}_{2}\right)(\mathrm{OH})_{6} 8 \mathrm{H}_{2} \mathrm{O}$ is $1.5(9 / 6)$ and 2.25 for dimer. Tobermorite has a $\mathrm{C} / \mathrm{S}$ of 0.83 for infinite chain and 1.25 for dimer (Taylor, 1986). Figure 28 shows the relationship between $\mathrm{Ca} / \mathrm{Si}$ ratio and silicate chain for Tobermorite and Jennite. At a chain length of two, the $\mathrm{Ca} / \mathrm{Si}$ ratio is 1.25 and 2.25 , respectively, and when the chain length is infinite, the ratio is 0.8 and 1.5 , respectively. The $\mathrm{C}-\mathrm{S}-\mathrm{H}$ has a mean $\mathrm{Ca} / \mathrm{Si}$ ratio of 1.75 , which is between the value of dimer of Tobermorite and dimer of Jennite. 


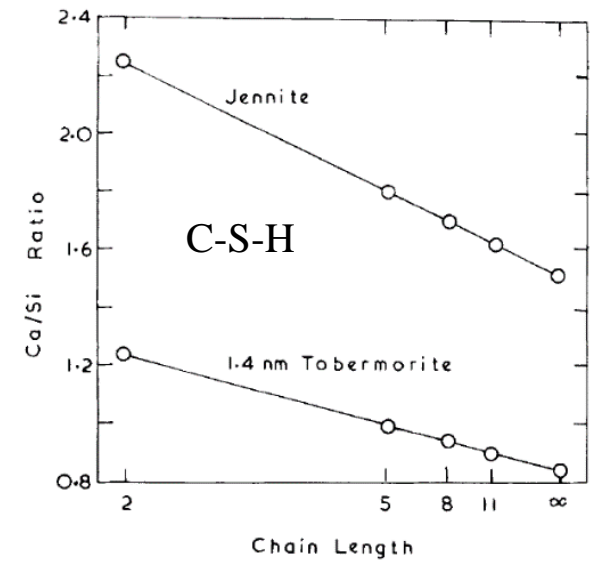

Figure 28: C/S ratio vs chain length (Taylor H. F., 1986)

The mean silicate chain in C-S-H increases with time, and dimer are formed during the early age of hydration, while pentamer form at later ages. At early age of cement hydration, $\mathrm{C}-\mathrm{S}-\mathrm{H}$ can be similar to Torbermorite and Jennite-like structures, with dimer chain and C/S ratios of 1.25 to 2.25 , respectively, as shown in Figure 28. This analogy has become the base of all other proposed models. Some researchers proposed another model based on the Taylor model. It is called Tobermorite/Portlandite, and in it, Tobermorite is sandwiched between Portlandite (Richardson, 2004) to increase C/S ratio. Other authors have proposed Tobermorite with more $\mathrm{Ca}^{2+}$ and $\mathrm{OH}^{-}$.

Richardson (2004) proposed a generalized model which includes the Tobermorite-Jennite $(\mathrm{T} / \mathrm{J})$ and $(\mathrm{T} / \mathrm{CH})$ models. It also accounts for the substitution of $\mathrm{Al}^{3+}$ for $\mathrm{Si}^{4+}$ ions at the bridging tetrahedral. The formulation of this model is provided by the following formula:

$$
\left\{\mathrm{Ca}_{2 \mathrm{n}} \mathrm{H}_{\mathrm{w}} \mathrm{Si}_{(3 \mathrm{n}-1)} \mathrm{O}_{(9 \mathrm{n}-2)}\right\} \cdot(\mathrm{OH})_{\mathrm{w}+\mathrm{n}(\mathrm{y}-2)} \cdot \mathrm{Ca}(\mathrm{n} \cdot \mathrm{y}) / 2 \cdot \mathrm{mH}_{2} \mathrm{O}
$$

w: number of silanol; w/n: degree of protonation of silicate chain.

\subsection{Other hydration products}

\subsubsection{Portlandite}

Calcium hydroxide, also called Portlandite, forms from the hydration of $\mathrm{C}_{3} \mathrm{~S}$ and $\mathrm{C}_{2} \mathrm{~S}$, consist of 20$25 \%$ of the volume of the hydration paste. Portlandite has a crystalline structure, in contrast to C-S$\mathrm{H}$, which has an amorphous one. The morphology of Portlandite $(\mathrm{CH})$ is affected by space available 
for growth, temperature of hydration, and the presence of impurities and admixtures (Taylor, 1997). In general, Portlandite $(\mathrm{CH})$ has a hexagonal plate shape. In terms of mechanical contribution, it helps reduce pores in the paste, which has a small effect on paste strength. In addition, $\mathrm{CH}$ restrains the CS-H from shrinkage. One characteristic of Portlandite is its solubility, which can cause leaching of $\mathrm{CH}$ when it contacts with water and can give aggressive agents access to the paste. On the other hand, it has the ability to protect concrete reinforcement from corrosion by increasing alkalinity $(\mathrm{PH})$ in the system and creating a passive layer. The structure of Portlandite and its X-ray diffraction are shown in Figures 29 and 30, respectively.

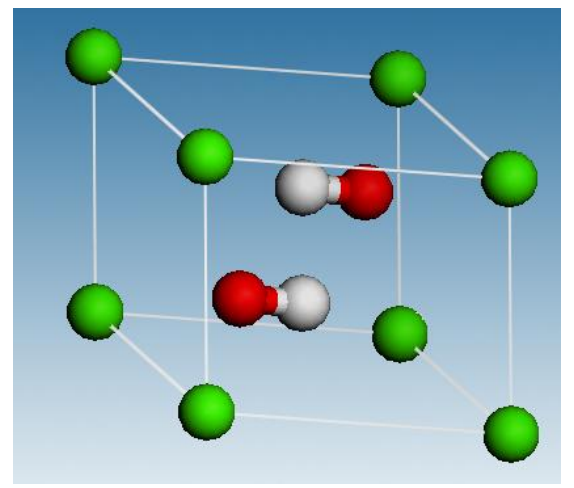

Figure 29: Unit cell Portlandite where $\mathrm{Ca}$ is presented in green, oxygen in red, hydrogen in white and the white line represents the unit cell

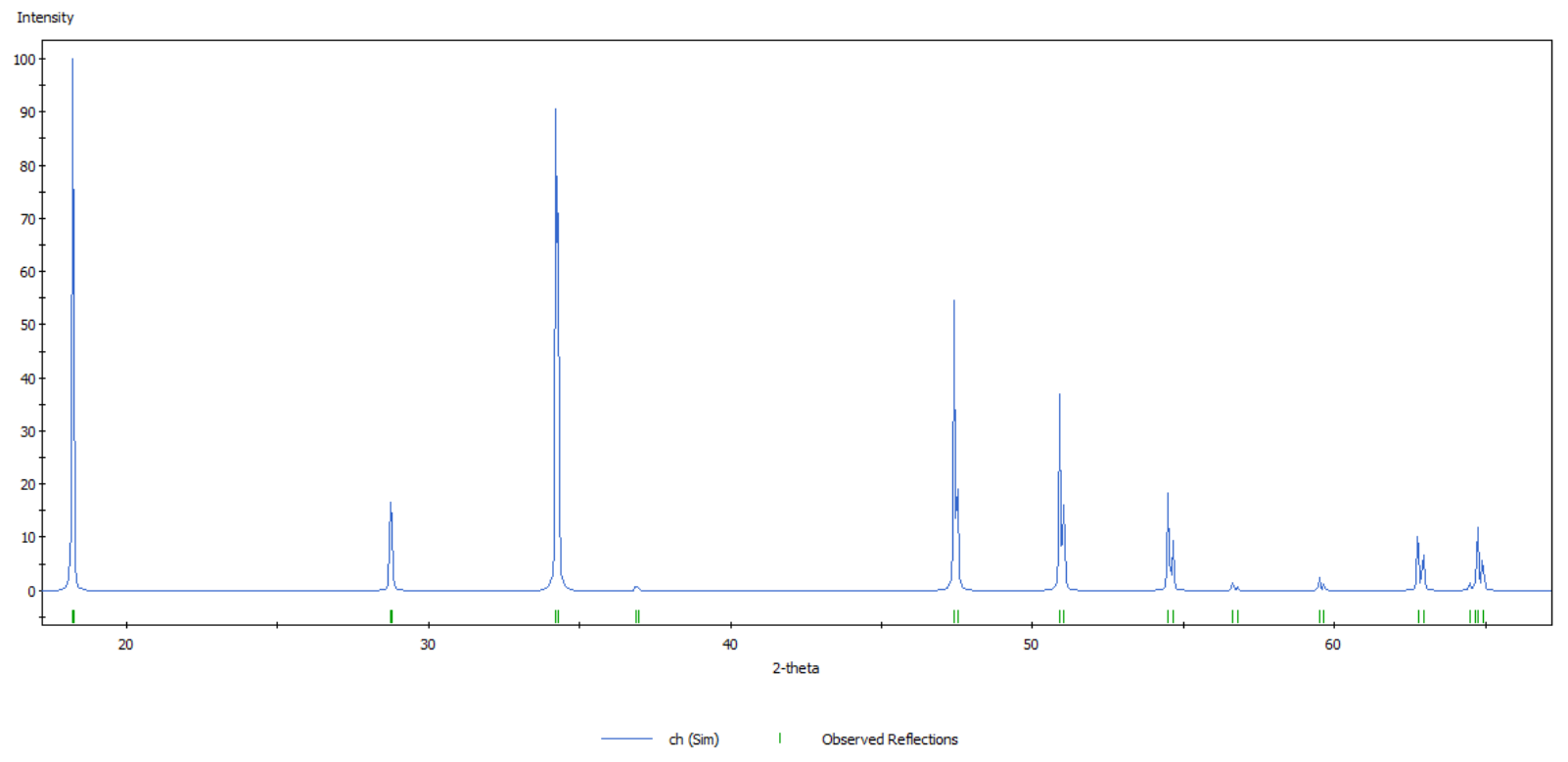

Figure 30: X-ray diffraction of Portlandite 


\subsubsection{Ettringite}

Ettringite $\left.\left[\mathrm{Ca}_{3} \mathrm{Al}(\mathrm{OH}) 612 \mathrm{H} 2 \mathrm{O}\right] 2(\mathrm{SO} 4) 3.2 \mathrm{H} 2 \mathrm{O}\right]$ is the hydration product of Portland cement formed in limited amounts during early hydration. Ettringite contributes to the mechanical properties and stability of cement paste. Excessive amounts of Ettringite can cause expansion of the paste, which leads to cracking. The morphology of Ettringite depends on the space available and the supply of ions for growth. It can have a needle shape with a high ratio of length over diameter. Ettringite is formed from the reaction of $\mathrm{C}_{3} \mathrm{~A}$ with gypsum:

$$
\mathrm{C}_{3} \mathrm{~A}+3 \mathrm{C} \overline{\mathrm{S}} \mathrm{H}_{2}+26 \mathrm{H} \rightarrow \mathrm{C}_{6} \mathrm{AS}_{3} \mathrm{H}_{32}
$$

\section{Tricalcium Aluminate Gypsum water Ettringite}

The unit cell and X-ray diffraction of Ettringite are shown in Figures 31 and 32, respectively.

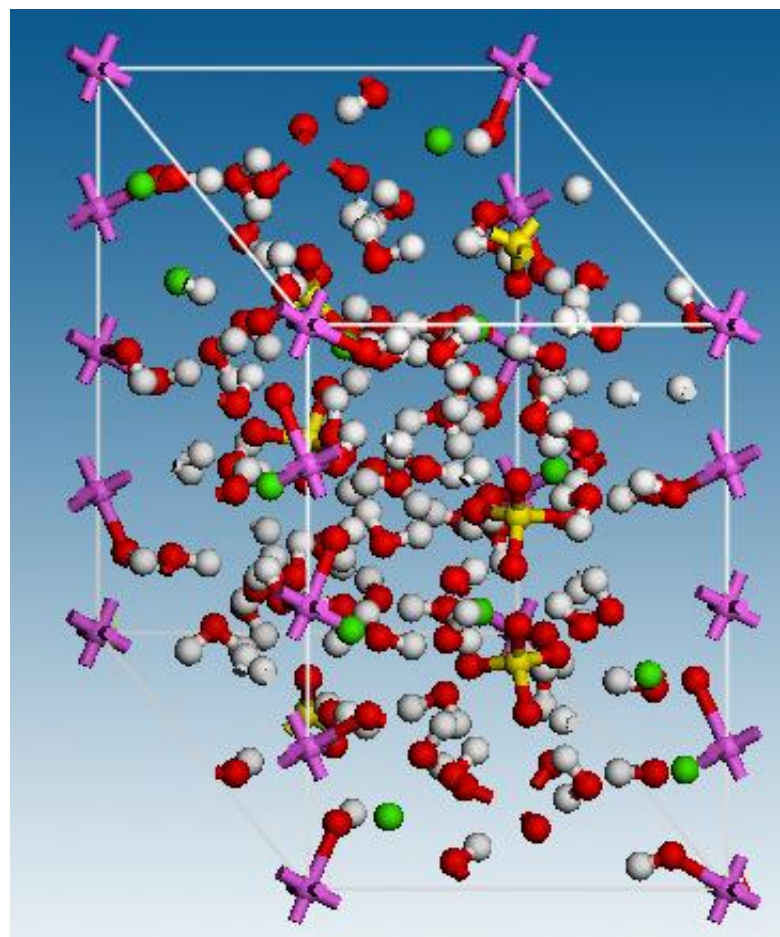

Figure 31: Unit cell Ettringite where $\mathrm{Ca}$ is presented in green, oxygen in red, hydrogen in white, aluminum in magenta and the white line represents the unit cell 


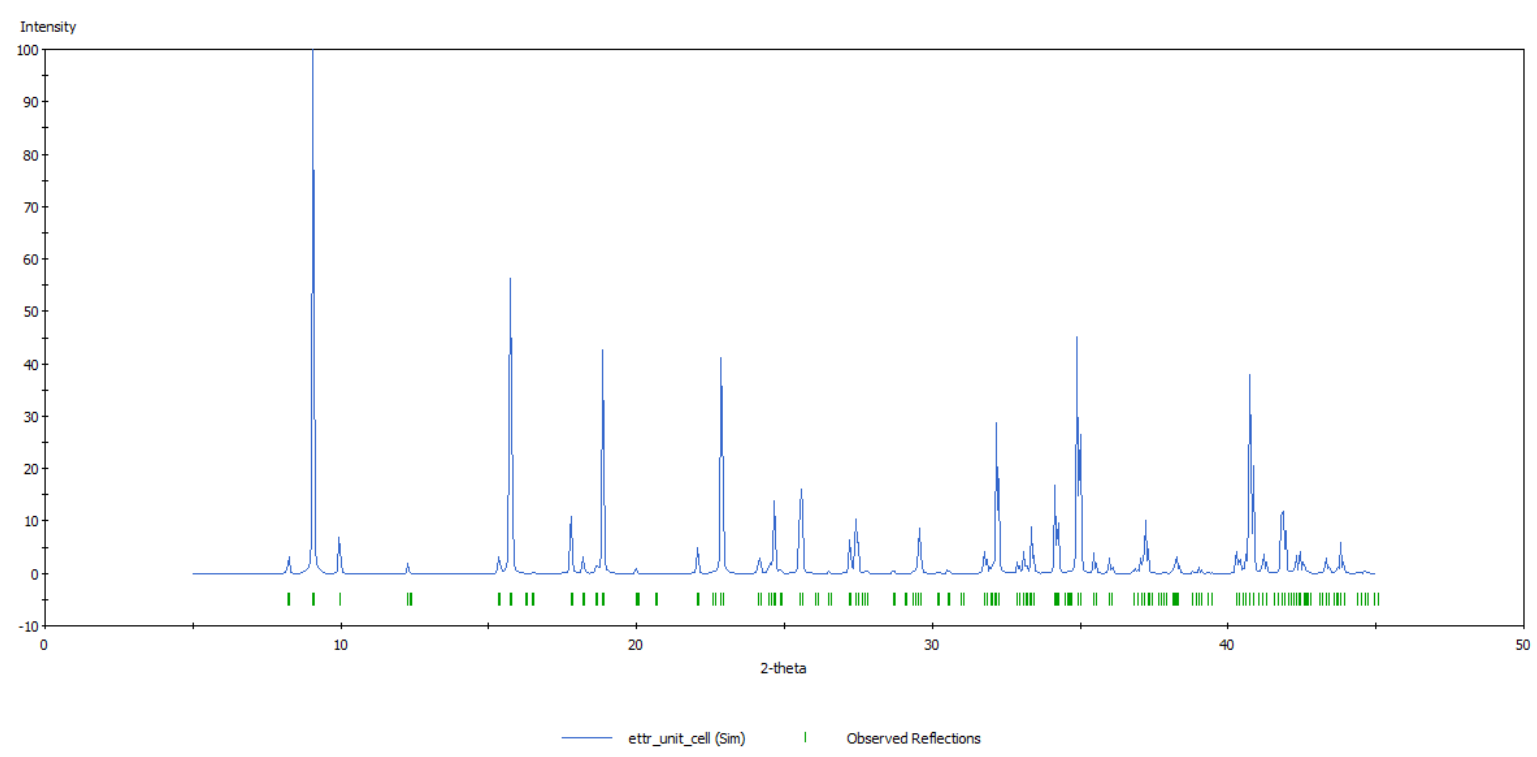

Figure 32: X-ray diffraction of Ettringite

\subsection{Summary}

This chapter present an overview of the clinker phases, hydration products of cement paste, similarities between C-S-H and Tobermorite and jennite, and models at nano-scale.

The major clinker phases are Alite $\left(\mathrm{C}_{3} \mathrm{~S}\right)$, Belite $\left(\mathrm{C}_{2} \mathrm{~S}\right)$, Tricalcium aluminate $\left(\mathrm{C}_{3} \mathrm{~A}\right)$ and Ferrite $\mathrm{C}_{4} \mathrm{AF}$. $\mathrm{C}_{3} \mathrm{~S}$ is the principal component of Portland cement clinker. It constitutes 50 to $90 \%$ of the clinker phases and is the most reactive composite. $\mathrm{C}_{2} \mathrm{~S}$ is the second principal clinker phase, and it makes up 10 to $40 \%$ of its composition. $\mathrm{C}_{3} \mathrm{~A}$ and $\mathrm{C}_{4} \mathrm{AF}$ are the minor components in the clinker phases.

The chemical reaction between cement and water causes the setting and hardening of cement paste, a process called a hydration reaction. The hydration reaction is composed of several reactions that occur instantaneously or continuously. The degree of hydration is affected by a series of parameters such as the composition of the clinker phase, presence of foreign ions, surface area, w/c ratio, presence of SCM, chemical admixture and curing temperature. The hydration of $\mathrm{C}_{3} \mathrm{~S}$ and $\mathrm{C}_{2} \mathrm{~S}$ at normal temperatures leads to the formation of calcium silicate hydrate gel C-S-H and Portlandite $(\mathrm{CH})$. The hydration of $\mathrm{C}_{3} \mathrm{~S}$ controls setting and hardening at early ages, while the hydration of $\mathrm{C}_{2} \mathrm{~S}$ is slow, with full hydration taking several years. The hydration of $\mathrm{C}_{3} \mathrm{~A}$ includes reaction with sulfate ions (gypsum), resulting in the formation of Ettringite. If the gypsum is consumed before the complete 
hydration of $\mathrm{C}_{3} \mathrm{~A}$, the Ettringite reacts with the leftover $\mathrm{C}_{3} \mathrm{~A}$ to form monosulfoaluminate. The hydration of $\mathrm{C}_{4} \mathrm{AF}$ is similar to $\mathrm{C}_{3} \mathrm{~A}$.

Concrete, mortar and cement paste are heterogeneous materials that present a multi-scale level in which the properties change as the length scale changes. Concrete, mortar and cement paste can be divided into three levels: macro-scale, micro-scale and nano-scale. At the macro-scale level, the aggregate and cement paste are the major phases, along with macro pores (entrapped air) if they are formed. At the micro- and nano-scale levels, each phase of the macro level shows heterogeneity. For instance, the calcium silicate hydrate appears to be dense in some areas (high density) (HD) and shows low density (LD) in others. In addition to C-S-H phases, Portlandite (CH) also can be detected in the capillary voids.

Although the structure of $\mathrm{C}-\mathrm{S}-\mathrm{H}$ is amorphous and is not yet solved, some techniques such as nuclear magnetic resonance (NMR), scanning electron microscope (SEM), small-angle X-ray scattering (SAXS) and nano-indentation tools assist researchers in determining some C-S-H characteristics. One of the most important characteristic of $\mathrm{C}-\mathrm{S}-\mathrm{H}$ is the molar ratio of $\mathrm{CaO}$ to $\mathrm{SiO}_{2}(\mathrm{C} / \mathrm{S})$, which is in the range of 1.2 to 2.3 with a mean of 1.75 . With the nano-indentation tool, researchers can determine the mechanical properties of the two intrinsic types of C-S-H, which are LD C-S-H and HD C-S-H (low density and high density). The mean Young modulus of LD C-S-H and HD C-S-H are estimated to be between 20GPa and 30GPa. In addition, LD C-S-H and HD C-S-H show a packing factor of 0.64 and 0.74 respectively.

$\mathrm{C}-\mathrm{S}-\mathrm{H}$ has a layer structure in which the silicate chain follows the $3 \mathrm{n}-1$ order. As hydration progresses, monomer numbers decrease and the dimer numbers increass. Higher chains such as pentamar and octamer are formed at late ages. Based on previous $\mathrm{C}-\mathrm{S}-\mathrm{H}$ modeling, a relationship has been established between C/S ratio and the mean silicate chain, in which the mean silicate chain increases when the $\mathrm{C} / \mathrm{S}$ ratio decreases.

Tobermorite and Jennite are the structures that show the greatest similarities with C-S-H. Tobermorite can be found in three forms: Tobermorite 14A, 11A and 9A. Tobermorite 14A transforms to 
Tobermorite $11 \mathrm{~A}$ and $9 \mathrm{~A}$ upon heating. Its structure is composed of $\mathrm{SiO}_{4}$ tetrahedrons in a linear chain in a dreierkette form that repeats at every third tetrahedral, and a central layer of $\mathrm{CaO}_{2}$ in which oxygen is shared with the $\mathrm{SiO}_{4}$ and the interlayer is filled with water and calcium. Jennite also has a structure similar to Tobermorite, with the presence of the $\mathrm{OH}$ group in the sublayer $\mathrm{CaO}-\mathrm{OH}$ and the absence of $\mathrm{SiOH}$ at the terminal. The $\mathrm{C} / \mathrm{S}$ ratios of Tobermorite $14 \mathrm{~A}$ and Jennite are equal to 0.83 and 1.5 , respectively. 


\section{Chapter 3 Computational Methods}

\subsection{Introduction}

The purpose of molecular modeling is to mimic the behavior of molecules with the use of computers to predict their behavior and properties at the molecular level. It has a wide range of applications for different disciplines such as material science, chemical engineering, and engineering science. Atomic simulation is the result of the solution of a mathematical model that estimates the position and response of electrons and nuclei. This model is based on two approaches: quantum and classic mechanics. The quantum approach is based on the solution of Schrödinger's equation, where atoms and molecules are modeled through a mathematical equation. Schrödinger's equation can be solved in two ways: the ab-intio method, where the properties of the structure are determined only by the solution of Schrödinger's equation with the use of quantum mechanics, and by a semi-empirical method used to simplify the solution of Schrödinger's equation using empirical parameters. Ab-initio method considers the electronic state and as result computation become expensive and rigorous. The semi-empirical method uses experimental results as an input to the mathematical model, with less computation time required than in the ab-initio method for a medium size system of 100 of atoms. The classic approach is based on classic physics and relies on the force field in which the parameters are embedded. It is a fast, useful method for large scale systems (with thousands of atoms), and does not deal with electronic properties. Also, it doesn't require experimental data from ab-initio for the parameters. 


\subsection{Molecular mechanics}

The molecular mechanics approach differs from the quantum mechanics approach in that the electrons and nuclei of the atoms are not explicitly included in the calculation. Molecular mechanics assumes molecules are a collection of masses that are connected to each other via springs of varying lengths (bond). These bond interactions can stretch, bend and twist. In addition, the non-bonded atoms interact with each other through Van der Waals and electrostatic interaction. The potential energy of the ensemble of atoms is calculated using Hooke's law. As a result of these simplifications, molecular mechanics is a fairly fast computational method, and is practical for small and large molecules alike. Molecular mechanics is based on the Born-Oppenheimer approximations, which state that the Schrödinger equation can be divided into two equations of motion: one that describes the motion of the electrons, and another that describes the motion of the nuclei. These motions can be studied separately (Leach, 2001). Molecular mechanics is used to calculate the energy surface of molecules as a function of the motion of the nuclei. In molecular mechanics, the bonds have an "equilibrium" of lengths and angles. Force field parameters are the value of bond and angle, and the corresponding force constants are used in the potential energy functions. Any deviation from the equilibrium results in an increase of total energy of the molecule. The total energy of the system is the summation of the individual energy given by:

\section{Energy $=$ Stretching Energy + Bending Energy + Torsion Energy + Out of plane bending Energy + Non-Bonded Interaction Energy}

\subsection{Potential functions}

\subsubsection{Stretching energy}

For simplicity, a harmonic oscillator was used to create a small vibration to calculate energy due to bond stretching from the equilibrium. A more accurate potential with higher terms can be used using the Taylor series. Morse potential is the most accurate for energy potential calculation. However, it has three parameters to calculate potential energy, which increases the computational cost for a 
system with a large number of atoms. In addition, most of the stretch bond does not generally deviate significantly from its equilibrium. As a consequence, a harmonic oscillator that uses Hooke's law is the best approach for energy calculation. Figure 33 shows bond stretching and Hook's law equation to calculate energy.

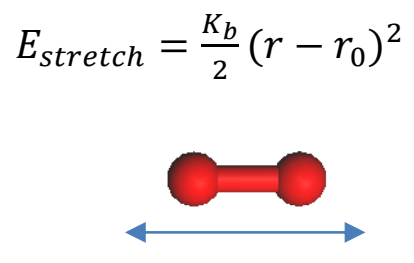

Figure 33: Bond stretching

Where $K_{b}$ is the stiffness of the bond spring, $r_{0}$ is the equilibrium bond length and $r$ is the current bond length after vibration.

\subsubsection{Bending energy}

Figure 34 shows the energy due to bending of the bond between three atoms joined in a series caused by the deviation of the angle from their reference. It can be calculated as follows:

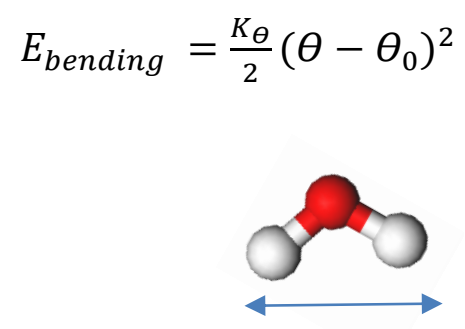

Figure 34: Angle bending

$K_{\theta}:$ is the force constant, $\theta_{0}$ : Angle at equilibrium, $\theta:$ Instantaneous angle

Less energy is required to distort the angle between the bonds than to stretch or compress a bond. A more accurate potential energy can be calculated using the Taylor series; it incorporates high order terms, which leads to a higher computational cost. 


\subsubsection{Torsion energy}

Most energy deviation is due to torsional and non-bonded energy contributions. Torsion energy is the result of deviation of the angle for a set of four atoms. Torsion energy is modelled by a simple periodic function, as shown in Figure 35.

$$
E_{\text {Torsion }}=\sum_{n=1}^{\infty} \frac{E}{2}(\cos n \varphi-\theta)
$$

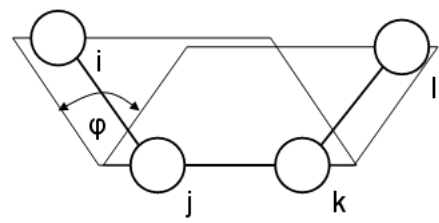

Figure 35: Bond torsion

Where E: barrier height, n: multiplicity, $\varphi$ torsion angle and $\Theta$ : phase shift

\subsubsection{Out of plane bending}

Deviation of the atom out of plane is treated differently than just bending on the plane. In out of plane, the three bending atoms are in one plane and the fourth atom is bent, making an angle $w$ with the plane. The energy deviation can be presented as shown in Figure 36 and expressed as harmonic function as follows:

$$
E_{\text {out plane }}=\frac{K_{\theta}}{2}(w)^{2}
$$

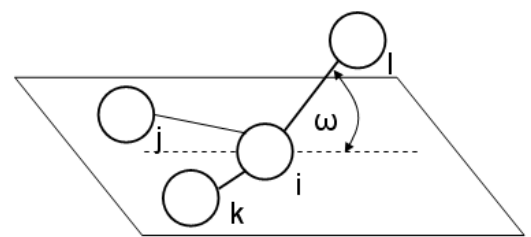

Figure 36: Out of plane

Where $K_{\theta}$ is the force constant, $w$ is the angular deviation from the plane.

\subsubsection{Non-bonded interactions}

Atoms that are not bonded to each other interact through non-bonded interaction forces. These interactions occur in space and are dependent on the distance between particles. Non-bonded 
interactions can be divided into two categories: electrostatic interactions and van der Waals interactions.

$$
\mathrm{E}_{\text {non-bonded }}=\mathrm{E}_{\text {electrostatic }}+\mathrm{E}_{\text {Van der waals }}
$$

\subsubsection{Electrostatic interactions (long range)}

Due to the difference in electronegativity between atoms, unequal distribution of charge is created. These charges produce an electrostatic force between atoms. The electrostatic interaction between two atoms $\mathrm{i}$ and $\mathrm{j}$ with partial charge is calculated using Coulomb's law.

$$
E_{\text {electros }}=\sum_{j=1}^{N a} \sum_{i=1}^{N b} \frac{q_{i} q_{j}}{4 \pi \varepsilon r_{i j}}
$$

Where $\mathrm{q}_{\mathrm{i}}$ and $\mathrm{q}_{\mathrm{j}}$ are the charges

$\mathrm{r}_{\mathrm{ij}}$ is the distance of the separation

$\mathcal{E}$ is the permittivity

$\mathrm{Na}, \mathrm{Nb}$ : number of punctual charges

Electrostatic interaction (Figure 37) has a slower rate of decay (1/r) than Van der Waals, which decays at a rate of $1 / \mathrm{r}^{6}$. It requires a considerable distance for decay, which leads to high computational cost.

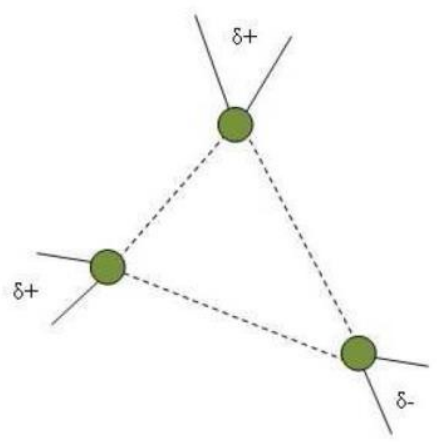

Figure 37: Electrostatic interactions 


\subsubsection{Van der Waals interactions (short Range)}

In addition to electrostatic interaction, Van der Waals interactions occur due to the induced dipole between two or more molecules. Van der Waals are the weakest interactions, however; due to the presence of many van der Waals interactions between atoms or molecules, they become very strong. The Van der Waals interaction has a short-range effect. As atoms move away from each other, energy decays rapidly at a rate of $1 / \mathrm{r}^{6}$. The van der Waals interaction energy is composed of two terms: attractive and repulsive. Repulsion occurs when the distance between two attracting particles becomes very small, which causes a high increase in energy due to an overlap of electrons in the space (Pauli). The attractive force occurs due to the instantaneous dipole from the fluctuation of electron clouds. The mathematical model that best describes this attraction and repulsion is called the Leonard Jones Potential [L-J] or 6-12, as shown in Figure 38 (Leach, 2001).

$$
E_{v d w}=4 \varepsilon\left[\left(\frac{\sigma}{r}\right)^{12}-\left(\frac{\sigma}{r}\right)^{12}\right]
$$

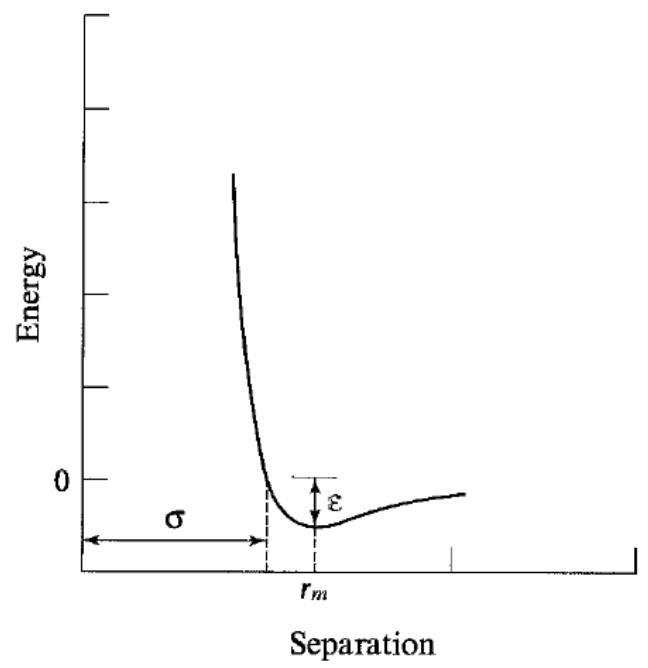

Figure 38: The Lennard-Jones potential (Leach, 2001)

\subsubsection{Force field used}

Force field is the functional form and parameter set used to calculate the potential energy of a system of atoms in molecular mechanics and molecular dynamics simulations. It is the core of material characterization using molecular mechanics. Different force field types such as UNIVERSAL, 
DREIDING and CVFF have been used in literature to simulate minerals such as clinker phases and hydration products. However, COMPASS, which is the most recent force field added to the Materials Studio software, has more reliable results compared to other force fields.

\subsubsection{COMPASS force field}

The force field used in molecular mechanics is the major parameter affecting the accuracy of the results. Among the different force fields used, COMPASS (Condensed-phase Optimized Molecular Potentials for Atomistic Simulation Studies), is the most recent force field added to the material software. It is a mixture of the ab-initio method and classic mechanics, which is the reason for its special characteristics and accurate predictions. It has been broadly used in a wide range of molecules and polymers.

$$
\begin{aligned}
& E_{\text {total }}^{\text {compass }}=E_{\text {valence }}+E_{\text {crossterm }}+E_{\text {non-bond }} \\
& =\sum_{b}\left\{K_{2}\left(b-b_{0}\right)^{2}+K_{3}\left(b-b_{0}\right)^{3}+K_{4}\left(b-b_{0}\right)^{4}\right\} \\
& +\sum_{\theta}\left\{H_{2}\left(\theta-\theta_{0}\right)^{2}+H_{3}\left(\theta-\theta_{0}\right)^{3}+H_{4}\left(\theta-\theta_{0}\right)^{4}\right\} \\
& +\sum_{\emptyset}\left\{V_{1}\left\{1-\cos \left(\emptyset-\emptyset_{1}^{0}\right)\right\}+V_{2}\left\{1-\cos \left(2 \emptyset-\emptyset_{2}^{0}\right)\right\}+V_{3}\left\{1-\cos \left(3 \emptyset-\emptyset_{3}^{0}\right)\right\}\right\} \\
& +\sum_{z} K_{z} X^{2}+\sum_{b} \sum_{b^{\prime}} F_{b b^{\prime}}\left(b-b_{o}\right)\left(b^{\prime}-b_{o}^{\prime}\right)+\sum_{\theta} \sum_{\theta \theta^{\prime}}\left(\theta-\theta_{o}\right)\left(\theta^{\prime}-\theta_{o}^{\prime}\right) \\
& +\sum_{b} \sum_{\theta} F_{b \theta}\left(b-b_{o}\right)\left(\theta-\theta_{o}\right)+\sum_{b} \sum_{\emptyset} F_{b \theta}\left(b-b_{o}\right)\left\{V_{1} \cos \emptyset+V_{2} \cos 2 \emptyset+V_{3} \cos 3 \emptyset\right\} \\
& +\sum_{b^{\prime}} \sum_{\emptyset} F_{b \theta}\left(b^{\prime}-b^{\prime}{ }_{o}\right)\left\{V_{1} \cos \emptyset+V_{2} \cos 2 \emptyset+V_{3} \cos 3 \emptyset\right\} \\
& +\sum_{\theta} \sum_{\emptyset} F_{b \theta}\left(\theta-\theta_{o}\right)\left\{V_{1} \cos \emptyset+V_{2} \cos 2 \emptyset+V_{3} \cos 3 \emptyset\right\} \\
& +\sum_{\emptyset} \sum_{\theta} \sum_{\theta^{\prime}} k_{\emptyset \theta \theta^{\prime}} \cos \emptyset\left(\theta-\theta_{o}\right)\left(\theta^{\prime}-\theta_{o}^{\prime}\right)+\sum_{i>j} \frac{q_{i} q_{j}}{\varepsilon r_{i j}}+\sum_{i>j}\left\{\frac{A_{i j}}{r_{i j}^{9}}-\frac{B_{i j}}{r_{i j}^{6}}\right\}
\end{aligned}
$$

Where: $\mathrm{K}, \mathrm{H}, \mathrm{F}$ and $\mathrm{B}$ are constant, $\mathrm{b}$ is bond distance, $\theta$ is the bond angle, $\Theta$ torsion angle and $\mathrm{V}$ is coefficient of coefficient of cosine Fourier expansion (Materials Studio software V7). 


\subsection{Energy Minimization}

Geometry optimization is the first important step in molecular computation. The purpose of a geometry optimization is to find a minimum energy potential surface with respect to atomic position. Any movement of the structure far from its equilibrium will generate a configuration with higher energy. The geometry of the molecule determines many of its physical and chemical properties (Ramachandran et al., 2008). The potential energy function can have many minimum values. The lowest is called the global energy minimum. To locate these energy minimum points, minimization algorithms are used. They can be categorized into two groups: one that uses a derivative of the energy function with respect to coordinates and one which does not. The direction of the derivative (positive or negative) indicates where the minimum energy is located, and the magnitude of the derivative indicates steepness. The energy of the system can be lowered by moving atoms to the relaxed position. The best algorithm is one that finds the minimum location as quickly as possible. Most algorithms move downhill of the surface energy, so they only locate the minimums that are nearest, as shown in Figure 39 and illustrated by the following function (Leach, 2001):

$$
F=-\frac{\partial E}{\partial r}
$$

E: potential energy, r: atom position

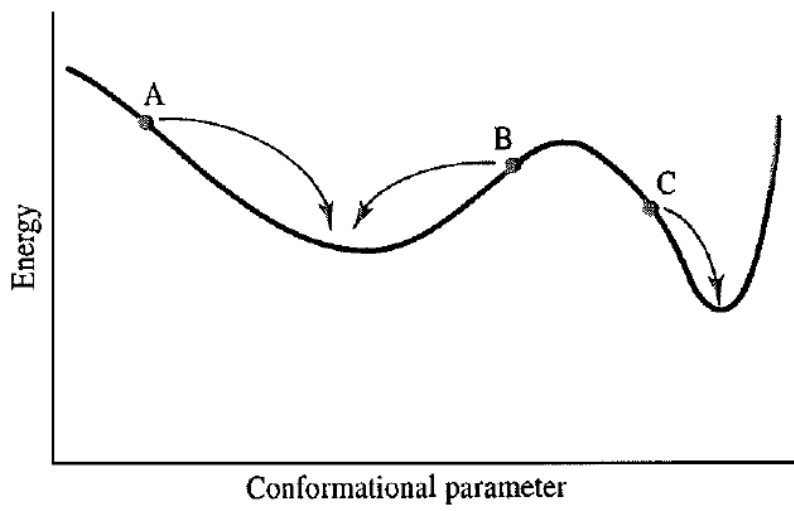

Figure 39: A schematic one-dimensional energy surface, minimization method moving downhill to nearest minimum (Leach, 2001) 


\subsubsection{Steepest Descent (SD)}

The Steepest Descent (SD) minimization algorithm uses the first derivative to find the position of atoms with minimum energy. The force derived shows the direction in which the atoms have to move towards the relaxed structure. The energy potential is calculated from the initial geometry; the atoms move with small increments in the direction of the force (called line of search) until the minimum is found in that direction. Another line search, perpendicular to the previous minimum point, locates the lowest minimum, as shown in Figure 40. The coordinates at step $i$ are:

$$
: x_{i+1}=x_{i}+\lambda x_{i}
$$
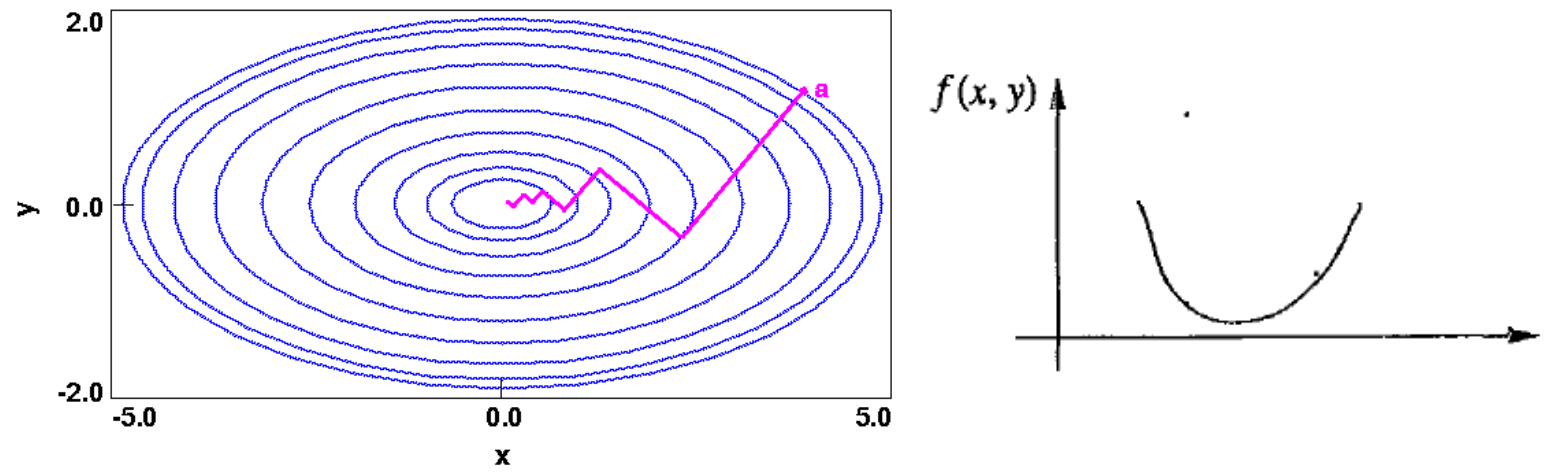

Figure 40: Minimization path followed by steepest-descents approach for simple quadratic function (Materials Studio)

This algorithm is effective when the initial configuration is far from the minimum. However, it will not be effective if the gradient of the potential is small.

\subsubsection{Conjugate gradient}

The Steepest Descent method converges slowly to the minimum because each line search segment must be orthogonal to the previous one, and therefore the progress made by the first line search is lost. In the conjugate gradient method, the direction mutually conjugates such successive steps to continue refining the direction towards the minimum. It uses both the current gradient and the previous search direction to drive minimization. Because the conjugated gradient method uses the minimization history to calculate search direction and contains a scaling factor for determining step 
size, this method converges faster and makes step sizes optimal as compared to the Steepest Descent technique. Figure 41 shows the minimization comparison between the steepest descent and conjugate gradient methods.

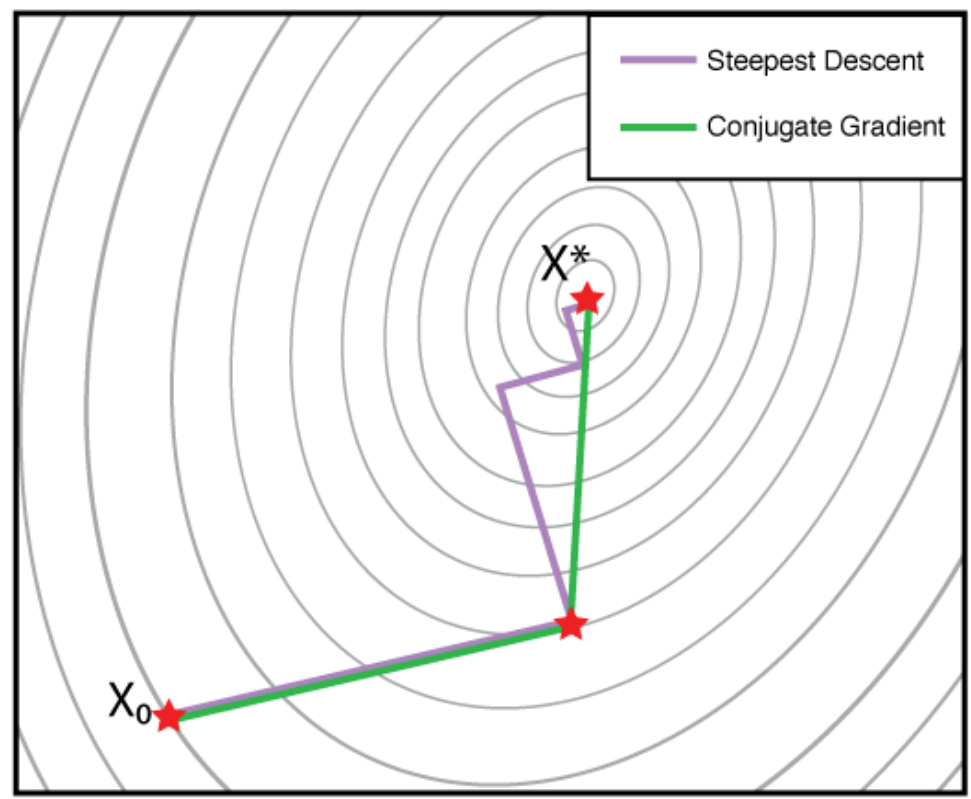

Figure 41: Minimization comparison between Steepest Descent and Conjugate gradient method (Materials Studio)

\subsection{Molecular dynamic}

Molecular dynamics is a computer simulation used to simulate the movement of atoms and molecules as they interact with each other (Frenkel \& Smit, 2002). The MD simulation produces a trajectory of the atoms and molecules that provides their positions and velocities in the system as a function of time by numerically solving Newton's law equation:

$$
\frac{d^{2} x_{i}}{d t^{2}}=\frac{F_{x i}}{m_{i}}
$$

Where $\mathrm{F}_{x i}$ is the force acting on the particles due to the interactions, $m i$ mass of the particles and $x i$ coordinates of atoms.

The purpose of molecular dynamics is to calculate the force between particles and their energy. MD is also used to determine the average properties of the system at a macroscopic level, which can be 
compared with experiments that have been conducted and also those that it is not possible to perform (Leach, 2001).

At a specified temperature, an initial random velocity is applied to the atoms, which causes the atoms to change position (trajectory). With potential (force field) used, the forces acting on the atoms can be determined at their new positions by:

$$
F_{i}=\frac{d E\left(r_{i}\right)}{d r_{i}(t)}=\nabla \mathrm{E}\left(\mathrm{r}_{\mathrm{i}}\right)
$$

Where $E$ is energy potential calculated from classic mechanics, $r i$ is the position of atoms. When velocity is applied to the set of molecules, the system will be out of equilibrium (thermodynamic equilibrium), and with time will be relaxed to a new value. When the system is brought to equilibrium, measurements of system properties can then be calculated. The force calculation is completed in a series of short steps (1 fs), and in each step the force, the new position and velocities are calculated until equilibrium is reached, the trajectory is obtained and average properties are calculated:

$$
<A>=\frac{1}{M} \sum_{i=1}^{M} A\left(P^{N}, r^{N}\right)
$$

Where $M$ is number of time steps, $P$ is momentum of atoms, $N$ number of atoms, $r$ is the position of the atom. 
The flow chart of the MD simulation algorithm is shown in Figure 42.

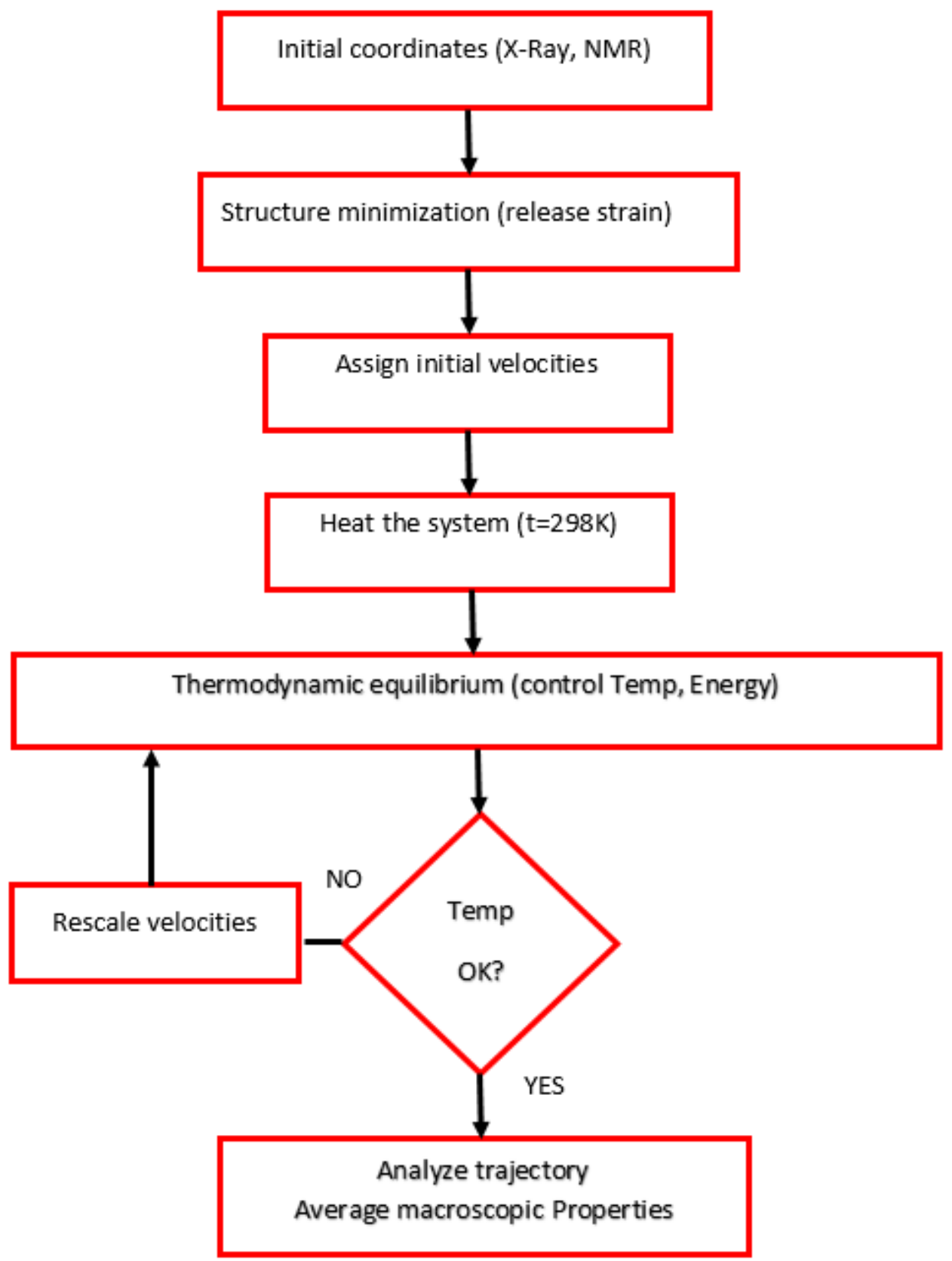

Figure 42: Flow chart of MD algorithm

\subsubsection{Integration of equation of motion (algorithms)}

The force on each particle will change as the particle changes its position, or the particles interacting with it change position. The motions of all particles are connected to each other, which creates a multi-body equation that cannot be solved analytically. Instead, the equation of motion is solved by using the finite difference method. The main idea is to break the integration into small steps at $\Delta \mathrm{t}$. 
The Verlet algorithm works in two steps: 1) use the current position of the particles to calculate the current force applied to the particles 2) use the current position $r_{n}$ and previous $r_{n-1}$ with the current force calculated in step 1 to calculate the position in the next step $r_{n+1}$. The algorithm is based on the third expansion of the Taylor series for forward and backward expansion, which calculates the position at $\mathrm{t}+\Delta \mathrm{t}$ (Becker et al., 2001).

$$
\begin{aligned}
\mathrm{r}(\mathrm{t}+\Delta \mathrm{t}) & =\mathrm{r}(\mathrm{t})+\Delta \mathrm{t} \mathrm{v}(\mathrm{t})+\frac{1}{2} \Delta t^{2} a(t)+\cdots \\
\mathrm{r}(\mathrm{t}-\Delta \mathrm{t}) & =\mathrm{r}(\mathrm{t})-\Delta \mathrm{t} \mathrm{v}(\mathrm{t})+\frac{1}{2} \Delta t^{2} a(t)+\cdots \\
(2.13)+(2.14) \quad \mathrm{r}(\mathrm{t}+\Delta \mathrm{t}) & =2 \mathrm{r}(\mathrm{t})-\mathrm{r}(\mathrm{t}-\Delta \mathrm{t})+\Delta \mathrm{t}^{2} \mathrm{a}(\mathrm{t})
\end{aligned}
$$

The velocity can be calculated as:

$$
\text { (2.13)- (2.14) } \quad \mathrm{v}(\mathrm{t})=[\mathrm{r}(\mathrm{t}+\Delta \mathrm{t})-\mathrm{r}(\mathrm{t}-\Delta \mathrm{t})] / 2 \Delta \mathrm{t}
$$

Figure 43 is a graphical representation of the steps followed by the Verlet algorithm:
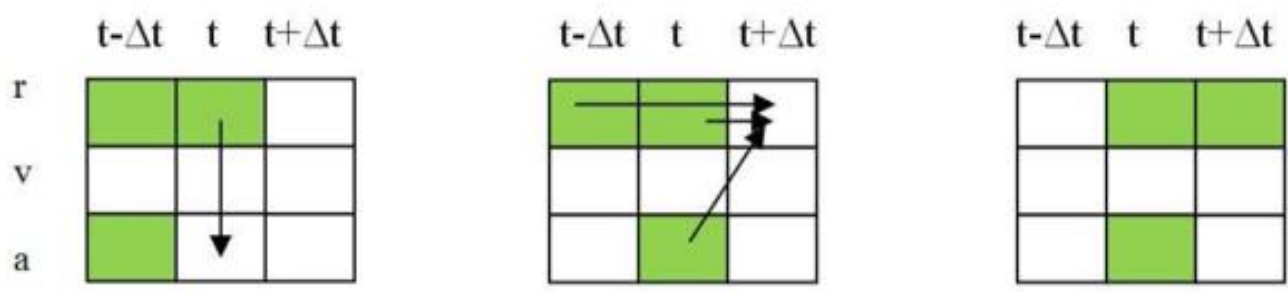

Figure 43 : Graphical representation of steps followed by Verlet algorithm (Allen and Tildesley, 1987)

\subsubsection{Periodic boundary condition (PBC)}

When we simulate a system, the atoms at the face of the simulated system have fewer neighbors than the atoms inside the box; the applied force on molecules at the surface is different than on those in the bulk. As a result, the system will not reflect the reality, no matter how large it is. Periodic boundary condition is used to solve this issue; in it, a system is replicated as an imaginary box to infinity in all Cartesian directions. During the simulation, as particles move in the original box, the particles in the periodic image will move the same way as in the original box. In addition, if one particle passes through the face of the original box, it reappears on the opposite face of the imaginary box with the 
same velocity, which leads to a constant number of particles in the box and maintained density.

During the simulations, the particles are free to cross the six cube faces, as shown in Figure 44.

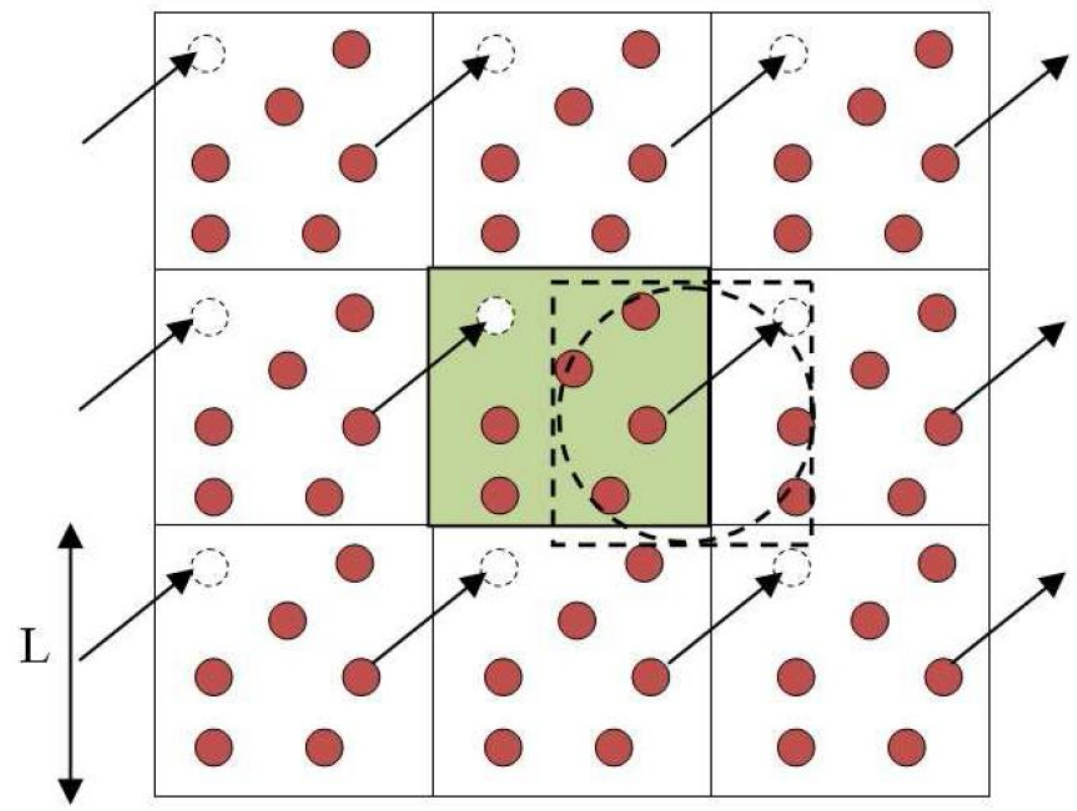

Figure 44: Periodic boundary conditions in molecular dynamics (Allen and Tildesley, 1987)

\subsubsection{Cutoff distance}

Due to the elimination of boundary conditions, the number of interactions between atoms increases, which leads to a reduced speed calculation. A cut-off distance is used to reduce the number of interactions, and any pair interaction beyond the cut-off distance is ignored. The cut-off distance should be less than half of the box dimension $(\mathrm{Cf}<\mathrm{L} / 2)$.

\subsubsection{Ensembles}

The purpose of molecular modelling is to determine the macroscopic properties of a system (pressure, energy, volume) through microscopic simulation. Statistical mechanics are able to determine the macroscopic properties from the individual atoms or molecules making up the system. At the macrocosmic level, a system can be defined by parameters such as temperature $(\mathrm{T})$, pressure $(\mathrm{P})$, Volume (V), and number of particles $(\mathrm{N})$. At the microscopic level, a system is defined by the position of atoms (q) and momenta (p), which are considered the coordinates in a three-dimensional space 
called phase space. An ensemble is a collection of all possible systems which have different microscopic states but identical macroscopic ones. Different ensembles can be used in molecular dynamic simulation.

In statistical mechanics, the ensemble average is given by:

$$
<A>=\iint A(p, q) P(q, p) d p d q
$$

$\mathrm{P}(\mathrm{q}, \mathrm{p})$ is the probability of the system to be at the phase space point $(\mathrm{q}, \mathrm{p})$, and $\mathrm{A}(\mathrm{q}, \mathrm{p})$ is the system property value at that point

\subsubsection{NVE}

A constant number of particles, constant volume and constant energy ensemble (NVE). The Newton equation of motion is solved without any temperature or pressure control.

\subsubsection{NVT}

A constant number of particles, constant volume, and constant temperature ensemble (NVT) maintain a constant temperature where atom velocities have to be controlled

\subsubsection{NPT}

A constant number of particles, constant pressure, and constant temperature ensemble (NPT) control both temperature and pressure. The unit cell is allowed to change and pressure is adjusted by adjusting the volume

\subsection{Mechanical Properties}

The most important mechanical properties of cement-based material are the short-term deformations controlled by the elastic modulus. The elastic properties describe how the material responds to small reversible deformation. The relationship between stress and strain can be written using Hooke's law:

$$
\sigma_{i}=\sum_{i=0}^{6} C i j \varepsilon j
$$

Where Cij are elastic tensor coefficients, $\varepsilon j$ is the strain. 
In molecular modeling, the elastic constants $\left(\mathrm{C}_{\mathrm{ij}}\right)$ can be calculated by the second derivative of energy density (Energy/Volume) with respect to lattice strain components.

$$
C_{i j}=\frac{1}{V}\left(\frac{\partial^{2} U}{\partial \varepsilon_{i} \partial \varepsilon_{J}}\right)
$$

Where: $\varepsilon_{\mathrm{i}}, \varepsilon_{\mathrm{j}}$ are lattice strain components, $U$ is potential energy, and $\mathrm{V}$ is simulation cell volume.

$$
\mathrm{Cij}=\left[\begin{array}{llllll}
C 11 & C 12 & C 13 & C 14 & C 15 & C 16 \\
C 21 & C 22 & C 23 & C 24 & C 25 & C 26 \\
C 31 & C 32 & C 33 & C 34 & C 35 & C 36 \\
C 41 & C 42 & C 43 & C 44 & C 45 & C 46 \\
C 51 & C 52 & C 53 & C 54 & C 55 & C 56 \\
C 61 & C 62 & C 63 & C 64 & C 65 & C 66
\end{array}\right]
$$

The elastic compliance matrix (S) can be obtained by finding the inverse matrix of Cij

\subsubsection{Bulk modulus and shear modulus}

Bulk $(\mathrm{K})$ and shear $(\mathrm{G})$ modulus can be calculated from the elastic tensor coefficient. There are three ways to calculate $\mathrm{K}$ and $\mathrm{G}$. The $\mathrm{K}$ and $\mathrm{G}$ in the Reuss are:

$$
\begin{aligned}
& K_{\text {Reuss }}=\left[\left(S_{11}+S_{22}+S_{33}\right)+2\left(S_{12}+S_{13}+S_{23}\right)\right]^{-1} \\
& G_{\text {Reuss }}=\frac{15}{4}\left[\left(S_{11}+S_{22}+S_{33}-S_{12}-S_{13}-S_{23}\right)+3\left(S_{44}+S_{55}+S_{66}\right)\right]^{-1}
\end{aligned}
$$

The Voight definitions of $\mathrm{K}$ and $\mathrm{G}$ are:

$$
\begin{aligned}
& K_{\text {Voight }}=\frac{1}{9}\left[\left(C_{11}+C_{22}+C_{33}\right)+2\left(C_{12}+C_{13}+C_{23}\right)\right] \\
& G_{\text {Voight }}=\frac{1}{15}\left[\left(C_{11}+C_{22}+C_{33}\right)+3\left(C_{44}+C_{55}+C_{66}\right)-C_{12}-C_{13}-C_{23}\right]
\end{aligned}
$$

The bulk (K) and shear $(\mathrm{G})$ modulus from Reuss and Voight represent the upper and lower limits.

The average of the value of Reuss and Voight represents the Hill average

$$
\begin{aligned}
& K_{\text {Hill }}=\frac{K_{\text {Voight }}+K_{\text {Reuss }}}{2} \\
& G_{\text {Hill }}=\frac{G_{\text {Voight }}+G_{\text {Reuss }}}{2}
\end{aligned}
$$


When bulk and shear have been calculated, Young modulus (E) and Poisson ratio are then calculated, assuming an isotropic material:

$$
\begin{aligned}
& E=\frac{9 G}{3+G / K} \\
& v=\frac{3-2 G / K}{6+2 G / K}
\end{aligned}
$$

\subsection{Summary}

This chapter describes different molecular computation methods and concepts used in literature. Two approaches are defined: molecular modeling based on quantum mechanics and molecular modeling based on molecular mechanics. One of the most famous methods that uses quantum mechanics is called ab-initio, which is based on the solution of the Schrödinger equation. Different techniques have been derived from this approach. However, because quantum mechanics consider the electronic state, the computation becomes expensive, rigorous and long. In the second approach, which uses classic (molecular) mechanics, the atoms are considered a collection of mass, connected with springs that permit the calculation of the potential energy of the system.

The force field used in molecular mechanics is a major parameter that can the reliability of results. COMPASS force field, the most recent force field used in cement paste simulation, has been previously used in different ranges of molecules and polymers simulations.

Molecular modeling with COMPASS force field is used to locate the points at which the energy of atoms is at its minimum; any movement from these points generates additional energy in the system. Energy minimization is used to locate the minimum energy of a system using algorithms such as Steepest Descent (SD), Conjugate Gradient and others. Energy minimization is also used as a preequilibrium before running molecular dynamics. 
Molecular dynamics simulate the progress and interaction of atoms over a period of time. The Verlet algorithm is used to numerically solve the Newton equation of motion. Molecular dynamics act as a bridge between microscopic and macroscopic scales in which we can obtain bulk properties.

Elastic constants can be calculated by the numerical second derivative of energy density (Energy/Volume) with respect to lattice strain components. 


\section{Chapter 4 Elastic Properties of major clinker phases}

\subsection{Introduction}

When cement is mixed with water, it results in a paste in which the different crystal phases are composed from reacted and unreacted cement. The reacted components, called hydration products, are mainly composed of calcium silicate hydrate $(\mathrm{C}-\mathrm{S}-\mathrm{H})$ and Portlandite $(\mathrm{CH})$, in which $\mathrm{C}-\mathrm{S}-\mathrm{H}$ is the main contributor to the mechanical properties of the cement paste. The unreacted components $\left(\mathrm{C}_{3} \mathrm{~S}, \mathrm{C}_{2} \mathrm{~S}, \mathrm{C}_{3} \mathrm{~A}, \mathrm{C}_{4} \mathrm{AF}\right)$ are composed mainly from the clinker phase and have high percentages of Alite and Belite. The anhydrate (unreacted) phase can be found in early ages of cement paste, or in mature ages when W/C ratio is low. The anhydrate phase in this case can also affect global mechanical properties. Knowing the properties of these different phases is important to improving paste quality. Calculating mechanical properties such as Young modulus, Poisson ratio, bulk modulus and shear modulus is important at the nano-scale level to determine properties at macro- and micro-scale levels.

\subsection{State of art}

Velez et al. (2001) performed experiments using the nano-indentation method at the microscopic level to determine the elastic modulus and hardness of synthetic $\mathrm{C}_{3} \mathrm{~S}, \mathrm{C}_{2} \mathrm{~S}, \mathrm{C}_{3} \mathrm{~A}$ and $\mathrm{C}_{4} \mathrm{AF}$. They also used resonance frequency measurement at the macroscopic level. The structure and purity of the synthesized phases were analyzed by X-ray diffraction. In the nano-indentation experiment, the penetration depth of the indenter was about 300 to $500 \mathrm{~nm}$, with an applied load of 40 to $50 \mathrm{mN}$. They 
found that the elastic modulus of different synthetic clinker phases is in the range of 125 to $145 \mathrm{GPa}$ (Velez et al. (2001)). They also observed no significant change in Young modulus between $\mathrm{C}_{3} \mathrm{~S}$ and Alite which has some impurities. The same applied for $\mathrm{C}_{2} \mathrm{~S}$ and Belite. For resonance frequency testing, specimens with different densities were made to account for porosity. The elastic modulus (as a function of porosity) was calculated by measuring the resonance frequency of the bar specimen $(10 \times 1 \times 2 \mathrm{~cm})$, and they found that the elastic modulus at zero porosity was higher, in a range of 140 to 160GPa, for all clinker phases. They also concluded that elastic modulus decreases about 30 to $40 \%$ when porosity increases from 10 to $20 \%$. For $\mathrm{C}_{3} \mathrm{~S}$, the fit equation for porosity is

$$
E=146.89 * e^{-3.4 p}
$$

Mondal (2008) performed experiments to determine the variation of the elastic modulus of C-S-H throughout the surface. He used a triboindenter instrument, which combines nano-indentation and scanning probe microscopy (SPM) imaging. The advantage of this instrument is its ability to locate the indenter in a specific location with a high-resolution image, identify the different phases of the cement paste, and position the indenter probe within 10nm (Mondal 2008). Mondal (2008) found that Young modulus of the anhydrate cement paste is around 100GPa, which is slightly lower than the Velez results (Velez et al., 2001). They also found that there are three groups of elastic modulus that decrease with distance from the anhydrate cement. The elastic modulus map using nano-indentation is presented in Figure 45.
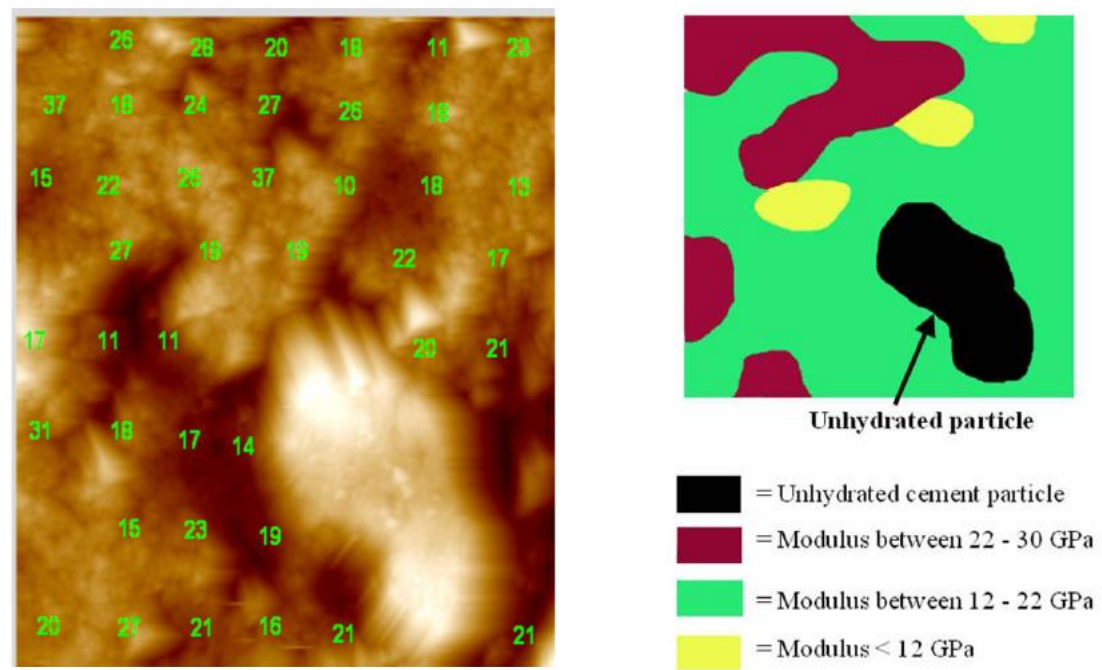

Figure 45: Elastic modulus map using nano-indentation (Mondal, 2008) 
The elastic modulus map is based on the elastic modulus of each indentation. Four groups of elastic modulus were found: anhydrate cement, low stiffness $\mathrm{C}-\mathrm{S}-\mathrm{H}$, high stiffness areas, and very low stiffness areas due to C-S-H porosity.

\subsection{Basic review of crystal structure}

A crystal is buildup from a repeating structural motif (Atkins, 1986), which can be atoms or molecules. A unit cell is an imaginary parallelepiped that contains one unit of the repeating pattern. The length of the unit cell is a, b and $\mathrm{c}$, and the angles between them are $\alpha, \beta$ and $\gamma$. A unit cell can be classified into seven crystal systems: triclinic, monoclinic, orthorhombic, rhombohedral, tetragonal, hexagonal and cubic. Table 1 shows the different types of crystals including cubic, monoclinic, triclinic and their axial parameters (length and angles) a, b, c, $\alpha, \beta$, and $\gamma$, as presented in Figure 46.

Table 1: Crystal system with lattice parameters

\begin{tabular}{lll}
\hline Crystal family & Crystal system & Axial relationships \\
\hline Isometric & Cubic & $a=b=c, \alpha=\beta=\gamma=90^{\circ} ;$ \\
Tetragonal & Tetragonal & $a=b \neq c, \alpha=\beta=\gamma=90^{\circ} ;$ \\
Orthorhombic & Orthorhombic & $a \neq b \neq c, \alpha=\beta=\gamma=90^{\circ} ;$ \\
Monoclinic & Monoclinic & $a \neq b \neq c, \alpha=90^{\circ}, \beta \neq 90^{\circ}, \gamma=90^{\circ} ;$ \\
Anorthic & Triclinic & $a \neq b \neq c, \alpha \neq 90^{\circ}, \beta \neq 90^{\circ}, \gamma \neq 90^{\circ} ;$ \\
Hexagonal & Hexagonal & $a=b \neq c, \alpha=\beta=90^{\circ}, \gamma=120^{\circ} ;$ \\
& Trigonal or & $a=b=c, \alpha=\beta=\gamma ;$ or \\
& Rhombohedral & $a^{\prime}=b^{\prime} \neq c^{\prime}, \alpha^{\prime}=\beta^{\prime}=90^{\circ}, \gamma^{\prime}=120^{\circ} ;$ \\
& & (hexagonal axes) \\
\hline
\end{tabular}

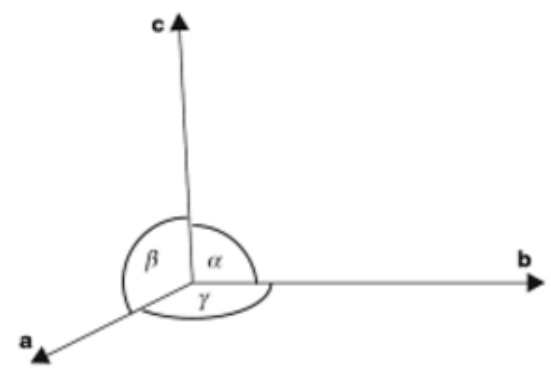

Figure 46: Crystal structure showing angles in the cell 


\subsection{Computation method}

The goal in this study was to use COMPASS force field instead the other current force fields in the Materials Studio software (Materials Studio v7.0). Elastic properties of all the anhydrate phases of cement were calculated by using COMPASS force field as the first choice. However, COMPASS was not applicable for all the clinker phases; DREIDING and CVFF in the Materials Studio software was also used when COMPASS was not applicable. Before starting molecular dynamic simulation, it is a prerequisite to do an energy minimization of the structure to remove any excess energy in the system. Minimization (or optimization of the geometry) is an iterative process in which the coordinates of the atoms and cell parameters are adjusted and bring the structure to a minimal energy. Minimization leads to a structure that is similar to the real physical structure. In addition, when performing a geometry optimization, temperature and pressure are not considered. However, they are controlled in molecular dynamics (MD). Materials Studio software uses a smart minimizer algorithm, which starts with steepest descent and is followed by the conjugated gradient algorithm (Materials Studio v7.0). After energy minimization of the structure, the dynamic simulation was performed in two steps: equilibrium and production. At the beginning of the equilibrium phase, a random velocity was assigned to all atoms in the structure at a selected target temperature. The temperature was controlled during the equilibrium phase by nose thermostat, and the pressure was controlled by Anderson barostat. Molecular dynamics can be completed in a different ensemble: NVE, NVT, NPH and NPT. All molecular dynamics simulations in this study were completed in the NPT ensemble, in which N is the number of atoms, $\mathrm{V}$ is the volume of periodic cell, $\mathrm{E}$ is the total energy is temperature, $\mathrm{P}$ is the pressure and $\mathrm{H}$ is enthalpy. The equilibration stage was used to equilibrate the kinetic and potential energies. The kinetic energy was increased due to the heating of the system from $0^{\circ} \mathrm{C}$ to $300^{\circ} \mathrm{C}$, and had to be equilibrated by potential energy. As soon as kinetic energy is equal to the potential energy, equilibration is reached. Plotting energy/temperature vs. time determines whether the system has reached that stage. When quantities such as energy and temperature fluctuated around their average, the system reached its equilibrium, as shown in Figures 47 and 48. 
Fordite Dynamics Energies

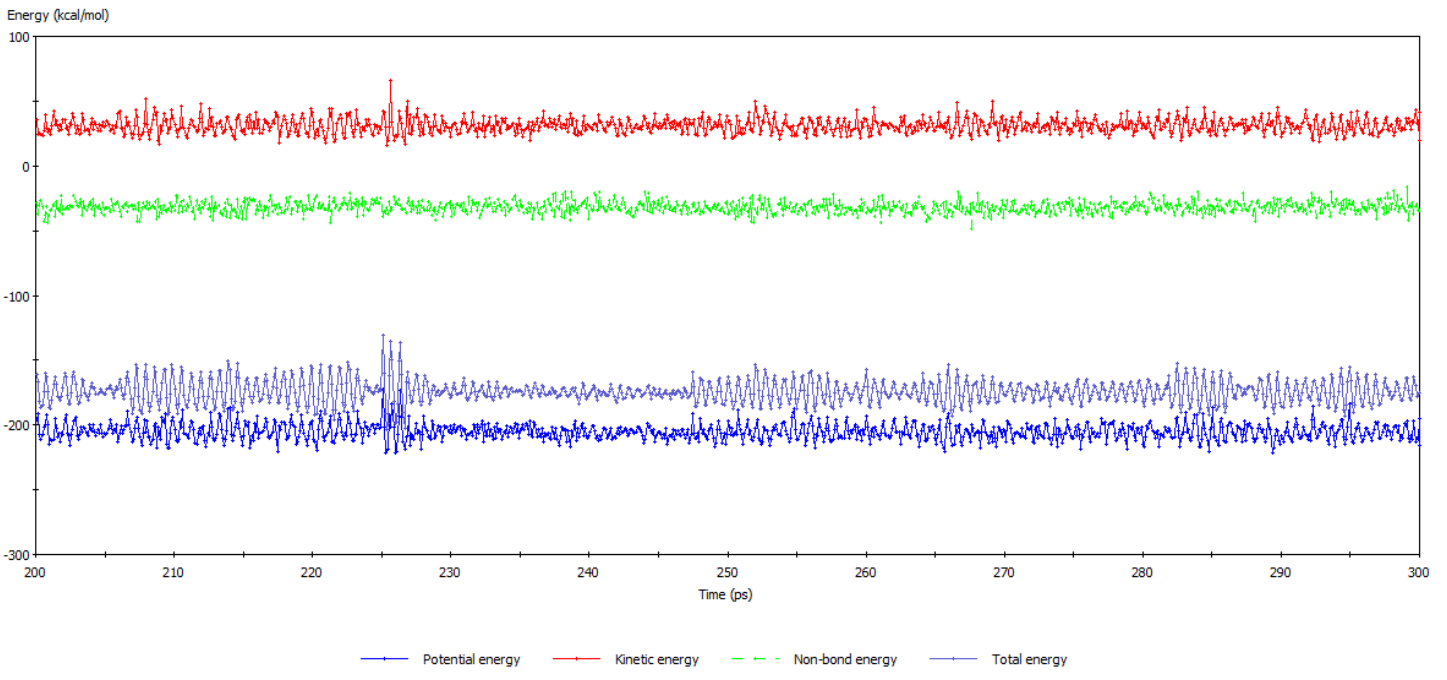

Figure 47: Potential energy, kinetic energy, non-bonded energy and total energy fluctuation during equilibration phase

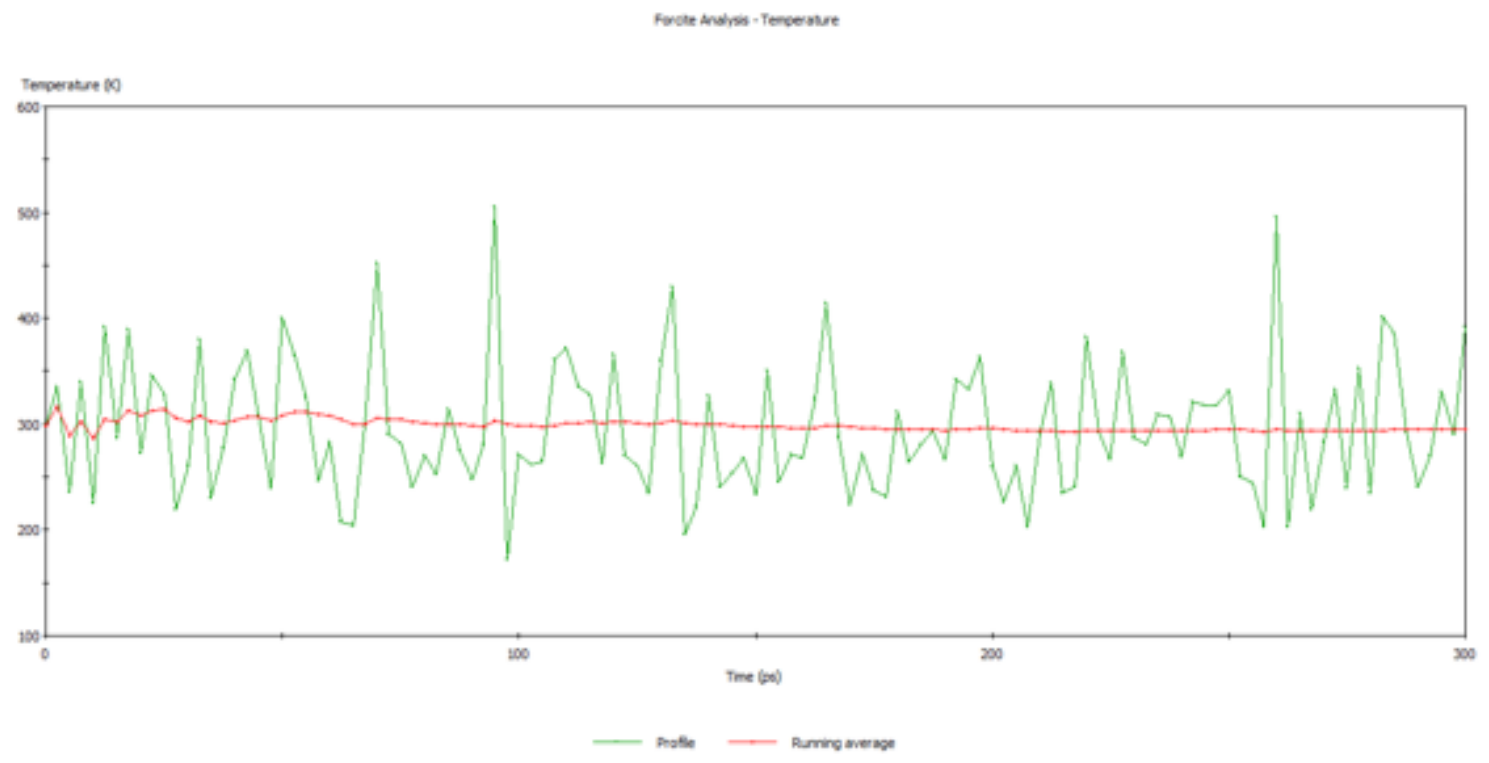

Figure 48: Fluctuation of temperature and running average temperature during equilibration phase

When the equilibrium of the system reached the desired temperature (298k) and pressure, the production run started and data and statistics were collected. Another important parameter in MD simulation is the time step used in algorithm integration; too large a step can lead to inaccurate results. NPT ensemble was used in all simulation in this study.

\subsubsection{Crystal parameters}

Table 2 shows the lattice parameters ( $a, b, c, \alpha, \beta$, and $\gamma$ ) of the clinker phases used in this study. 
Table 2: Unit cell parameters for different clinker phases

\begin{tabular}{ccccc}
\hline Crystal lattice & $\mathbf{C}_{3} \mathbf{S}$ & $\boldsymbol{\beta}-\mathbf{C}_{2} \mathbf{S}$ & $\mathbf{C}_{3} \mathbf{A}$ & $\mathbf{C}_{4} \mathbf{A F}$ \\
\hline $\mathbf{a}$ & 11.6389 & 5.480 & 15.263 & 5.584 \\
$\mathbf{b}$ & 14.1716 & 6.760 & 15.263 & 14.600 \\
$\mathbf{c}$ & 13.6434 & 9.280 & 15.263 & 5.374 \\
$\boldsymbol{\alpha}$ & 104.982 & 90.000 & 90.000 & 90.000 \\
$\boldsymbol{\beta}$ & 94.622 & 94.330 & 90.000 & 90.000 \\
$\boldsymbol{\gamma}$ & 90.107 & 90.000 & 90.000 & 90.000 \\
$\mathbf{R e f}$ & {$[1]$} & {$[2]$} & {$[3]$} & {$[4]$}
\end{tabular}

Ref: reference

[1]: (Torre et al., 2008)

[2]: (Midgley, 1952)

[3]: (Mondal and Jeffery, 1975)

[4]: (Jupe et al., 2001)

[5]: (Pedersen and Semmingsen, 1982)

\subsubsection{Geometry minimization}

An empirical approach was used based on COMPASS force field, where applicable, to determine interactions between atoms. However, this targeted force field was not applicable for all clinker phases. After creating the cell with the atoms connected, a geometry optimization was run to bring the structure to its relaxed state. Because COMPASS force field showed limitations in the case of $\mathrm{C}_{3} \mathrm{~A}$ and $\mathrm{C}_{4} \mathrm{AF}$, DREIDING and CVFF force fields, respectively, were used for those two phases. Table 4 shows the parameters used to optimize the structure.

Table 3: Unit cell parameters for different clinker phases

\begin{tabular}{cc}
\hline \hline \multicolumn{2}{c}{ Geometry minimization parameters } \\
\hline \hline Force field & COMPASS, DREIDING , CVFF \\
Quality & Ultrafine \\
Summation Method & Ewald \\
Repulsive cutoff & $12.5 \mathrm{~A}-18.5 \mathrm{~A}$
\end{tabular}




\subsubsection{Dynamic simulation parameters}

After minimizing the structure, a dynamic simulation was run where the atoms interacted with each other for a period of time. The position of the atoms was determined by numerically solving the Newton equation, and the forces between atoms were calculated with the potential energy using the force field mentioned in Table 4. Table 5 shows the parameters used in dynamic simulations.

Table 4: Dynamic simulation parameters

\begin{tabular}{cc}
\hline \hline \multicolumn{2}{c}{ Dynamic Simulation parameters } \\
\hline \hline Ensemble & NPT \\
Temperature & $298 \mathrm{~K}\left(25^{\circ} \mathrm{C}\right)$ \\
Pressure (GPa) & 0.0001 \\
Time step (fs) & 0.5 \\
Total simulation \\
(ps) \\
Thermostat \\
Barostat & $200-500$ \\
\end{tabular}

\subsection{Computation results}

When the forces between atoms are calculated, the mechanical properties of the structure can be determined. Table 5 shows the mechanical properties of the clinker phases from atomic simulation compared with other theoretical and experimental results from literature. 
Table 5 : Elastic properties of cement clinker: bulk modulus, shear modulus and Young modulus, Poisson ratio and experimental results (Appendix I, II, III and IV)

\begin{tabular}{ccccc}
\hline \hline Crystal & $\mathrm{C}_{3} \mathrm{~S}$ & $\mathrm{C}_{2} \mathrm{~S}$ & $\mathrm{C}_{3} \mathrm{~A}$ & $\mathrm{C}_{4} \mathrm{AF}$ \\
\hline \hline Bulk Modulus :K(GPa) & 162.3 & 64.6 & 176.1 & 127.8 \\
Shear modulus: $\mathrm{G}(\mathrm{GPa})$ & 50.04 & 50.6 & 64.2 & 82.9 \\
Young modulus: E(GPa) & 136.1 & 120 & 171.7 & 204.5 \\
v : Poisson ratio & 0.36 & 0.19 & 0.34 & 0.234 \\
Forcefield used & $\mathrm{FC}$ & $\mathrm{FC}$ & $\mathrm{FD}$ & $\mathrm{FCV}$ \\
Ref results (GPa) & & & & \\
$\mathrm{E}$ & $135 \pm 7^{[6]}$ & $130 \pm 20^{[6]}$ & $160 \pm 10^{[6]}$ & $125 \pm 25^{[6]}$ \\
$\mathrm{K}^{[8]}$ & 103 & 111 & 133.26 & 175.6 \\
$\mathrm{G}^{[8]}$ & 54.5 & 53.1 & 63.9 & 93.5 \\
$\mathrm{E}^{[8]}$ & 138.9 & 137.9 & 165.1 & 238.2
\end{tabular}

[6]: (Velez et al., 2001)

[7]: (Bass, 1995)

[8]: (Manzano et al., 2009)

FC: Force field COMPASS

FD: Force field DREIDING

FCV: Force field CVFF

The atomic simulations of $\mathrm{C}_{3} \mathrm{~S}, \mathrm{C} 2 \mathrm{~S}, \mathrm{C}_{3} \mathrm{~A}, \mathrm{C} 4 \mathrm{AF}$ structures were conducted using a regular desktop computer instead of the powerful computer typically used by researchers. The bulk and shear modulus were computed with Reuss, Voight and Hill equations, as explained in section 2.21. The Reuss method gives the upper bound and Voight represents the lower bound, while the Hill method represents the average of both. Only the results from the Hill method are presented in this section; Reuss and Voight results appear in the appendix. Young modulus and Poisson ratios were calculated from the Hill bound.

\subsection{Micro properties of the unreacted phase}

Unreacted particles in the cement paste contain porosity due to grain packing. The elastic properties of C-S-H depend significantly on the scale level. For nano-scale, the structure includes nano pores, which normally have a low influence on mechanical properties. However, at micro-scale, the effect of micro pores is estimated to be relevant. The micro properties of the unreacted phase can be 
calculated by using a packing factor density, which is defined by the ratio of volume of solid to total volume. Kepler (Askeland et al., 2011) proposed a packing ratio of spheres that presents the densest possible packing:

$$
\mathrm{\eta}_{\text {Kepler }}=\frac{\pi}{\sqrt{18}}=74.048 \%
$$

The porosity is then calculated by:

$$
p=1-\eta_{\text {Kepler }}=26 \%
$$

One of the simplest equations used to calculate the elastic modulus (taking porosity into account) is provided by Knudsen and Helmuth (Yoshimura et al., 2007).

$$
\begin{aligned}
& E_{\text {knudsen }}=E_{0} e^{(-k p)} \\
& E_{\text {Helmuth }}=E_{0}(1-p)^{k} \quad k=3
\end{aligned}
$$

Where $E_{o}$ is elastic modulus with zero porosity, $p$ is the porosity and $k$ fitting factor from experimental results. $\mathrm{k}=3.4$ (Velez, et al., 2001)

$$
E=E_{0} e^{(-3.4 p)}
$$

Table 6 shows the Young modulus results of the clinker phases, calculated After applying the different porosities on $\mathrm{C}_{3} \mathrm{~S}, \mathrm{C}_{2} \mathrm{~S}, \mathrm{C}_{3} \mathrm{~A}$ and $\mathrm{C}_{4} \mathrm{AF}$ structures.

Table 6: Elastic modulus of clinker phases with porosity

\begin{tabular}{rcccc}
\hline \hline Clincker phase & $\mathbf{C}_{3} \mathbf{S}$ & $\mathbf{C}_{2} \mathbf{S}$ & $\mathbf{C}_{3} \mathbf{A}$ & $\mathbf{C}_{4} \mathbf{A F}$ \\
\hline Eo $(\mathbf{G P a})$ & $\mathbf{1 3 6}$ & $\mathbf{1 2 0}$ & $\mathbf{1 7 1}$ & $\mathbf{2 0 4 . 5}$ \\
Eknudsen $(\mathbf{G P a})$ & & & & \\
Porosity: $(p=0.1)$ & 96.8 & 85.41 & 121.71 & 145.56 \\
$(p=0.15)$ & 81.66 & 72.06 & 102.68 & 122.80 \\
$(p=0.20)$ & 68.90 & 60.79 & 86.63 & 103.60 \\
$(p=0.25)$ & 58.12 & 51.29 & 73.09 & 87.41 \\
$(p=0.30)$ & 49.04 & 43.27 & 61.66 & 73.74 \\
$(p=0.35)$ & 41.37 & 36.51 & 52.02 & 62.21 \\
$(p=0.40)$ & 34.90 & 30.80 & 43.89 & 52.48 \\
$(p=0.45)$ & 29.45 & 25.98 & 37.03 & 44.28 \\
$(p=0.50)$ & 24.84 & 21.92 & 31.24 & 37.26 \\
$(p=0.55)$ & 20.96 & 18.49 & 26.36 & 31.52
\end{tabular}


After applying porosity to the elastic modulus resulting from the molecular dynamic simulation, remarkable decrements were noticed for all clinker phases. For instance, decrements of 29\%, 49\%, $64 \%, 74 \%$ and $82 \%$ were calculated when porosity of $10 \%, 20 \%, 30 \%, 40 \%$ and $50 \%$ was accounted for in the different clinker phases. Figure 49 displays the impact of porosity on the Young modulus results of $\mathrm{C}_{3} \mathrm{~S}, \mathrm{C}_{2} \mathrm{~S}, \mathrm{C}_{3} \mathrm{~A}$ and $\mathrm{C}_{4} \mathrm{AF}$.

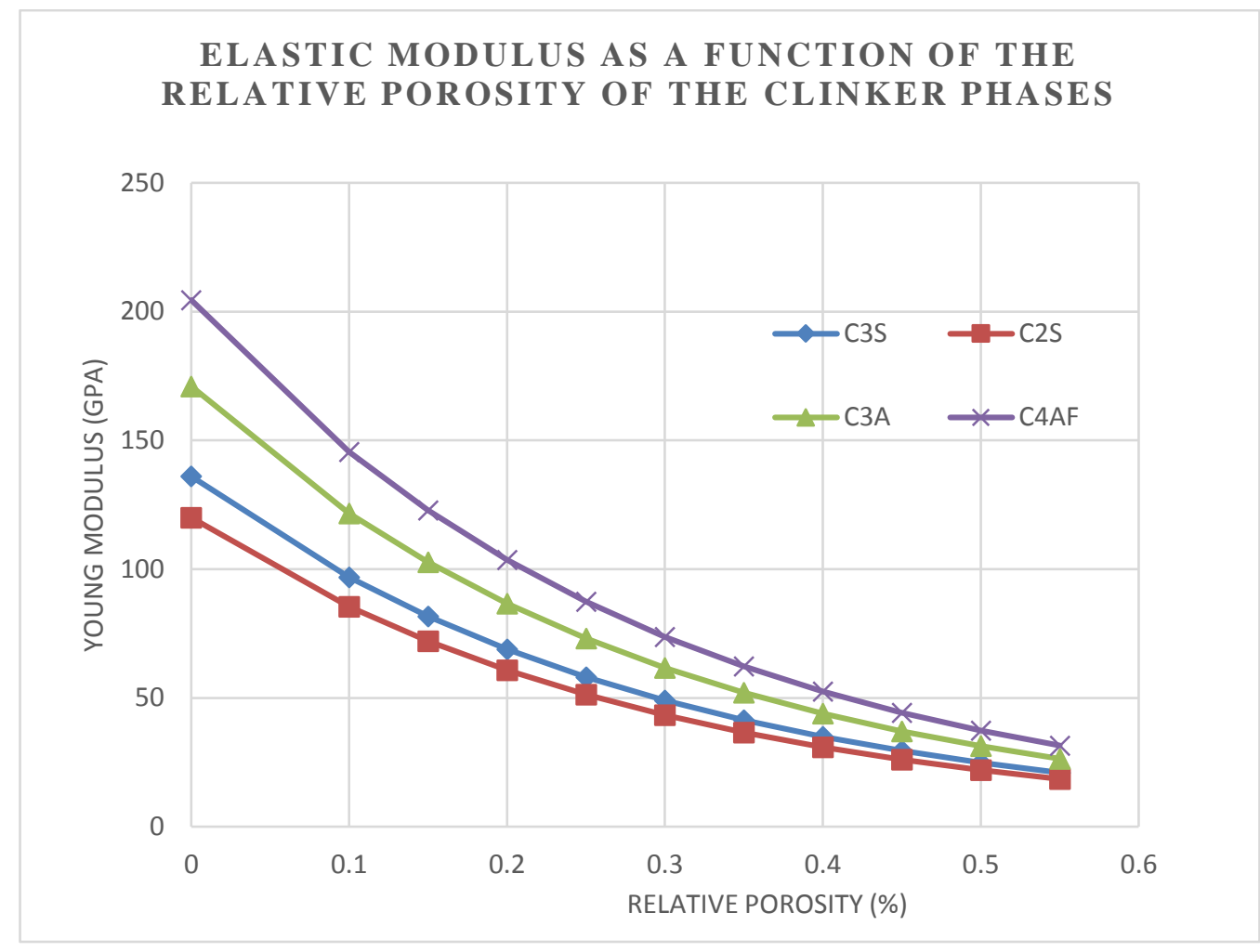

Figure 49: Elastic moduli as a function of the relative porosity of clinker phases

As shown in Figure 49, there was an inverse relationship between Young modulus and relative porosity, which is represented by an exponential function. As the porosity increased, the Young modulus decreased.

\subsection{Discussion of results}

The three major phases affecting the elastic modulus of cement paste are unreacted clinker particles, C-S-H and Portlandite $(\mathrm{CH})$. Unreacted cement grains can be present in the cement paste even After a long period of hydration, which can be due to the limited space for $\mathrm{C}-\mathrm{S}-\mathrm{H}$ to grow or to the low water to cement (W/C) ratio. All Young modulus results completed with molecular modeling using 
COMPASS and DREIDING force fields (C2S, C3S and C3A) agreed with the experimental results reported by Velez et al. (2001) and Bass (2015). For instance, molecular modeling of $\mathrm{C}_{3} \mathrm{~S}, \mathrm{C}_{2} \mathrm{~S}$ and $\mathrm{C}_{3} \mathrm{~A}$ showed values of $136.1,120$ and 171.7 , respectively, which is close to the experimental results that mention Young modulus of 135, 130, and 160, respectively. The only discrepancy that can be noted was for $\mathrm{C}_{4} \mathrm{AF}$, in which the calculated Young modulus (204.5 GP) was almost 1.6 times higher than the experimental value $(125 \mathrm{GPa})$ presented in the literature. However, this disagreement has already been mentioned by several researchers (Jorge et al., 2011), (Manzano et al., 2008). For example, Jorge et al. (2011), who used an ab-initio simulation, found a noticeable difference in the elastic modulus of the ferrite phase $\left(\mathrm{C}_{4} \mathrm{AF}\right)(238.2 \mathrm{GPa})$ compared to the experimental results (125GPa) presented by Velez et al., (2001). According to these authors, the reasons for the disagreement between the simulation results and the experimental values is not clear. However, in regard to the different force fields used in the present study, it can be assumed that the difference may be caused by the force field used $(\mathrm{CVFF})$ for $\mathrm{C}_{4} \mathrm{AF}$. Furthermore, the disagreement in the Young modulus of the ferrite phase could also have been caused by the number of cells used in the simulation. Indeed, in all the simulations completed in this work, only one cell was used to predict elastic properties. Using a higher number of cells in molecular modeling may require a more powerful computer with longer simulation times, which is incompatible with the reduced computation simulation time targeted in this study. Although the $\mathrm{C}_{3} \mathrm{~S}, \mathrm{C}_{2} \mathrm{~S}$ and $\mathrm{C}_{3} \mathrm{~A}$ results can be considered acceptable compared to the experimental values, further investigation is suggested for $\mathrm{C}_{4} \mathrm{AF}$.

When taking the effect of cement porosity on the elastic modulus of different clinker phases into consideration, the elastic modulus decreased as porosity increased, which is in agreement with the polynomial relationship mentioned by Powers (1960). For example, when increasing porosity from zero to $10 \%$ and $25 \%$, the Young modulus of different clinker phases decreased from $136.1 \mathrm{GPa}$ to 96.8 $\mathrm{GPa}$ and $68.9 \mathrm{GPa}$, respectively, which corresponds with decrements of $28.9 \%$ and $49.3 \%$, respectively. The decrease in the estimated Young modulus is also in agreement with the experimental results presented by Velez et al. (2001), which showed decrements of about $30 \%$ to $40 \%$ when 
porosity increased from $10 \%$ to $20 \%$ for $\mathrm{C}_{3} \mathrm{~S}, \mathrm{C}_{2} \mathrm{~S}$ and $\mathrm{C}_{3} \mathrm{~A}$ clinker phases. The porosity results also indicate that as the packing factor of grains decreases (and porosity increases), the elastic modulus decreases considerably. This relationship between packing factor and elastic modulus is an agreement with conclusions made by Constantinides and Ulm (2007), in which they showed that material became unstable when its packing factor reached a ratio of $0.5 \%$ (relative porosity of 0.5 ). They also found that the elastic modulus was mainly controlled by particle-to-particle contact, which can also explain our results.

One other point from the molecular simulation is the anisotropic elastic modulus shown in all clinker phases. For example, Alite had $\mathrm{E}_{\mathrm{X}}=145.4131, \mathrm{E}_{\mathrm{y}}=148.41$ and $\mathrm{E}_{\mathrm{z}}=122.61 \mathrm{GPa}$, as shown in appendix I. This confirms that the $\mathrm{C}_{3} \mathrm{~S}$ was more compressible in $\mathrm{Z}$ direction $(\mathrm{Ez}=122.61)$ than in $\mathrm{X}$ and $\mathrm{Y}$ directions $(E x=145.41$ and $E y=148.41)$. In the experimental results presented by Velez et al. $(2001)$ Young modulus direction was not taken into consideration, and Poisson's ratio of 0.3 was used for all constituents. However, this study shows that the low elastic modulus in $\mathrm{Z}$ direction can lead to higher expansion/shrinkage, shown by a high Poisson ratio $v_{\mathrm{zx}}$ of 0.3869 compared to $v_{\mathrm{yx}}=0.2755$ and $V_{y x}=0.2700$ (as shown in appendix I). This may illustrate that, in the case of $\mathrm{C}_{3} \mathrm{~S}, \mathrm{C}_{3} \mathrm{~A}$ and $\mathrm{C}_{2} \mathrm{~S}$, the results of molecular simulation can be considered more accurate than experimental results. Another notable result was the effect of $\mathrm{C} / \mathrm{S}$ ratio on the elastic modulus of clinker phases. $\mathrm{C}_{3} \mathrm{~S}$ and $\mathrm{C}_{2} \mathrm{~S}$ elastic modulus showed that the higher the $\mathrm{C} / \mathrm{S}$ ratio, the higher the elastic modulus. For example, $\mathrm{C}_{3} \mathrm{~S}$ had a $\mathrm{C} / \mathrm{S}=3$ and Young modulus of $136.1 \mathrm{GPa}$, while $\mathrm{C}_{2} \mathrm{~S}$ had a $\mathrm{C} / \mathrm{S}=2$ and Young modulus of $120 \mathrm{GPa}$. These results are investigated further in the following chapters.

\subsection{Summary}

The aim of the work outlined in this chapter was to simulate the elastic properties of clinker phases using molecular dynamic simulation by COMPASS force field. However, this targeted force field was applicable only to $\mathrm{C}_{3} \mathrm{~S}$ and $\mathrm{C}_{2} \mathrm{~S}$ structures, which led to the use of others force fields from 
Materials Studio software such as DREIDING and CVFF. The elastic properties determined by COMPASS and DREIDING force fields on $\mathrm{C}_{3} \mathrm{~S}, \mathrm{C}_{2} \mathrm{~S}$ and $\mathrm{C} 3 \mathrm{~A}$ showed agreement between the experimental values cited in literature and the estimated modulus resulting from this study. Although the CVFF molecular simulation of the $\mathrm{C}_{4} \mathrm{AF}$ structure showed an overestimated elastic modulus compared to the experimental values, the Young modulus of $\mathrm{C}_{4} \mathrm{AF}$ was estimated to be equal to 204.5 $\mathrm{GPa}$, which is close to the results of the ab-initio simulation.

When different porosity ratios were considered, results confirmed that elastic modulus decreased as porosity increased, which is in line with the experimental results mentioned in literature. 


\section{Chapter 5 Elastic Properties of Hydration products}

\subsection{Introduction}

Portlandite $\mathrm{Ca}(\mathrm{OH})_{2}$ is the second major hydration product of cement paste and constitutes $20-25 \%$ of its total volume. Knowledge of the elastic properties of Portlandite is important in the creation of cement paste. The presence of Portlandite in cement influences strength, reduces shrinkage and increases the alkalinity of the pore solution, which creates a passive layer that protects reinforcements from corrosion.

Ettringite is the third most relevant hydration product of Portland cement. It is a crystalline product that can affect the strength and long-term durability characteristics of concretes. The structure of Ettringite is composed of $\mathrm{Ca}_{6}\left[\mathrm{Al}_{2}(\mathrm{OH})_{12} .24 \mathrm{H} 2 \mathrm{O}\right]^{6+}$ placed on the top of each other in a $\mathrm{C}$ axis. One of the characteristics of Ettringite is its open structure, which allows interchange with other atoms. $80 \%$ of the atoms in Ettringite are water molecules and hydroxyl, which reduce its density to 1.7 and lead to a low elastic modulus (Speziale et al. 2008).

\subsection{State of art}

Elastic properties of Portlandite can be determined experimentally by X-ray spectroscopy and Brillouin zone spectroscopy. Speziale, et al. (2008) performed an experimental study on Portlandite using a natural specimen found in the Kalahari Manganese Field (South Africa). When they used Brillouin scattering measurements, they found that the Reuss and Voight bound of Portlandite were 
31.65 GPa and 20.95 GPa, respectively, and the Young modulus and Poisson ratios were 51.5 GPa and 0.23 .

Aierken et al. (2015) performed a study using a Density Function Theory (DFT) and experimental tools to determine the mechanical properties and other characteristics of $\mathrm{Ca}(\mathrm{OH})_{2}$. Due to the fact that $\mathrm{Ca}(\mathrm{OH})_{2}$ is a layered structure, they performed these calculations on the monolayer and bilayer of calcium hydroxide. As part of the experimental work, they produced the calcium hydroxide using $\mathrm{Ca}_{3} \mathrm{SiO}_{5}$ mixed with different water-to-solid ratios in the range of 0.2 to 0.9 and heated to $40^{\circ} \mathrm{C}$. The time of growth of calcium hydroxide was estimated to be 8 hours, with a water-to-solid ratio of 0.6 and a synthesized crystal dimension of 0.1-2 mm. To calculate the mechanical properties, they stacked these layers on the top of each other. The Young modulus of the calcium hydroxide was estimated to be 50.7 GPa and 55.0 GPa for the monolayer and bulk, respectively, whereas the shear modulus was 19.16 GPa and 21.23 GPa for the monolayer and bulk, respectively.

Speziale et al. (2008) also performed an experimental study using Brillouin spectroscopy to determine the elastic properties of Ettringite. They found the Young modulus was $25 \mathrm{GPa}$, the Hill average for the bulk modulus was $27.15 \mathrm{GPa}$ and the Hill average of shear modulus was $9.5 \mathrm{GPa}$. They concluded that the elastic tensor is strongly anisotropic, which relates to the lattice structure of mineral computation.

\subsection{Computational Method}

\subsubsection{Lattice parameters}

The structures of Portlandite and Ettringite used in this study were previously resolved by Henderson and Gutowsky (1962) and by Hartman and Berliner (2006), respectively. The lattice parameters (a, $\mathrm{b}, \mathrm{c}, \alpha, \beta$, and $\gamma$ ) reported by those authors and used in the simulation of this study are presented in Table 7 
Table 7: Lattice parameters for Portlandite and Ettringite

\begin{tabular}{ccc}
\hline Lattice Parameters & CH & Ettringite \\
\hline a & 11.6389 & 11.260 \\
$\mathbf{b}$ & 14.1716 & 11.260 \\
$\mathbf{c}$ & 13.6434 & 21.480 \\
$\boldsymbol{\alpha}$ & 104.982 & 90.000 \\
$\boldsymbol{\beta}$ & 94.622 & 90.000 \\
$\boldsymbol{\gamma}$ & 90.107 & 120.000 \\
$\boldsymbol{R}$ Re & {$[\mathrm{a}]$} & {$[\mathrm{b}]$}
\end{tabular}

[a]: (Henderson and Gutowsky, 1962)

[b]: (Hartman and Berliner, 2006)

The first step in our simulation was to build the crystal structure. To do so, the parameters mentioned in Table 7 were incorporated into the model data. ThereAfter, the atoms were added to the crystal depending on their types and coordinates.

\subsubsection{Geometry optimization parameters}

After trying COMPASS force field on both structures of Portlandite and Ettringite, it was decided that this type of molecular dynamic simulation is not applicable to the structure of Ettringite. For this reason, two force fields were used in this simulation: COMPASS force field for Portlandite and Universal for Ettringite. Table 8 shows the final parameters used to optimize the lattice structure.

Table 8: Geometry optimization parameters

\begin{tabular}{cc}
\hline \hline Geometry minimization parameters \\
\hline \hline Force field & COMPASS, Universal \\
Quality & Fine-Ultrafine \\
Summation Method & Ewald \\
Repulsive cutoff & $12.5 \mathrm{~A}$
\end{tabular}

\subsubsection{Dynamic optimization parameters}

Table 9 shows the parameters used in the dynamic simulation of Portlandite and Ettringite: 
Table 9: Dynamic optimization parameters

\begin{tabular}{cc}
\hline \multicolumn{2}{c}{ Dynamic Simulation parameters } \\
\hline \hline Ensemble & NPT \\
Temperature & $298 \mathrm{~K}\left(25^{\circ} \mathrm{C}\right)$ \\
Pressure (GPa) & 0.0001 \\
Time step (fs) & 0.5 \\
Total simulation (ps) & $100-500$ \\
Thermostat & Nose \\
Barostat & Anderson
\end{tabular}

After minimizing the structure, the data mentioned in Table 9 were incorporated. The dynamic simulation was run in which the atoms interacted with each other for a period of time. The position of atoms was determined by numerically solving the Newton equation, whereas forces between atoms were calculated with the potential energy using different force fields.

\subsection{Dynamic simulation results}

When the different forces between atoms are calculated, the mechanical properties of the crystals can be determined. Table 10 shows the mechanical properties of Portlandite and Ettringite resulting from the simulation in this study. For comparison purposes, some experimental results mentioned in literature are also presented in Table 10.

Table 10: MD simulation results of Portlandite and Ettringite (Appendix VI)

\begin{tabular}{lcc}
\hline \multicolumn{1}{c}{ Crystals } & CH & Ettringite \\
\hline \hline Bulk Modulus :K(GPa) & 46.3 & 15 \\
Shear modulus: G(GPa) & 17.8 & 7.33 \\
Young modulus: E(GPa) & $\mathbf{4 7 . 3}$ & $\mathbf{1 8 . 9}$ \\
$\mathbf{v}$ : Poisson ratio & 0.329 & 0.29 \\
Force field used & FC & FU \\
Ref $\quad$ E $(\mathbf{G P a})$ & $\mathbf{5 1 . 3 5}^{[\mathrm{c}]}, \mathbf{5 5}^{[\mathrm{d}]}$ & $\mathbf{2 5}^{[\mathrm{e}]}$
\end{tabular}




\section{FC: Force field COMPASS \\ FU: Force field Universal \\ [c]: (Speziale et al., 2008) (1) \\ [d]: (Aierken et al., 2015) \\ [e]: (Speziale et al., 2008)(2)}

The bulk modulus, shear modulus, Young modulus and Poisson ratio were obtained after running the dynamic simulation, as shown in Table 10. Those mechanical characteristics were the final results targeted in our simulation.

\subsection{Discussions}

After minimizing the structure of calcium hydroxide and Ettringite, the calculation of elastic modulus reported in this section was carried out by molecular dynamic simulation, using COMPASS and Universal force fields in Materials Studio software. Young and shear modulus estimated for Portlandite (47.3 GPa and 17.8 GPa, respectively) were slightly lower than the experimental results reported by Speziale et al. (2008) (which were $51.35 \mathrm{GPa}$ and 20.95GPa), who used Brillouin spectroscopy (section 4.1). In addition, the average bulk modulus found in this study (46.3GPa) was slightly higher than the result found by Speziale et al. (2008), which was $31.65 \mathrm{GPa}$. This small inconsistency can be related to the accuracy of the experimental tools used to measure the elastic properties, in which the porosity may not have been accounted for. Another reason may be that COMPASS force field was used to determine Portlandite structure. It is well known from the literature that the type of force field is the principal parameter affecting the results of the molecular dynamic simulation.

Although small differences were noted compared to the experimental results mentioned in literature, the results of Portlandite agreed with the elastic properties found by Aierken et al. (2015), who used the first principle calculation method. For example, Aierken et al. (2015) calculated Young modulus of 55GPa, bulk modulus of $44 \mathrm{GPa}$, shear modulus of $21.23 \mathrm{GPa}$ and Poisson ratio of 0.30 , which are close to the results of this study. 
Ettringite results shown in Table 11 indicate bulk, shear and Young modulus of $15 \mathrm{GPa}, 7.33 \mathrm{GPa}$, 10 and $18.9 \mathrm{GPa}$, respectively. These values are slightly lower than the experimental results completed by Speziale et al. (2008), which showed bulk, shear and Young modulus of 27.15 GPa, 9.5 GPa and $25 \mathrm{GPa}$, respectively. However, the results of the current study agree with the other simulation methods shown in literature; for instance, Kamali et al. (2004) determined elastic modulus of $22.4 \mathrm{GPa}$ and a Poisson ratio of 0.25 by using the ELAS3D modeling program.

When considering the structure proposed by Hartman and Berliner (2006) used in this study, Ettringite is composed of $[\mathrm{Al}(\mathrm{OH}) 6]^{3-}$ and water molecules with sulfate ions. As shown in Figure 31, section 1.13.2 (Chapter 1), $[\mathrm{Al}(\mathrm{OH}) 6]^{3-}$ linked through $\mathrm{Ca}^{2+}$ run parallel to $\mathrm{c}$ axis, while water molecules with sulfate ions are in the inner part of the structure. In the Ettringite formula ([Ca기 $\left.\left.\left.(\mathrm{OH}) 612 \mathrm{H}_{2} \mathrm{O}\right] 2(\mathrm{SO} 4) 3.2 \mathrm{H} 2 \mathrm{O}\right]\right)$, most of the atoms are water molecules or hydroxyl, which result in a reduced Ettringite density estimated at 1.79 (Materials Studiov7). This result agrees with the experimental density of Ettringite (1.7) mentioned in section 4.2. The lower density of Ettringite can justify its low elastic modulus, estimated by using molecular dynamic simulation. The simulation results (shown in Appendix VI) indicate that the Young modulus of Ettringite in the three axes are: $\mathrm{Ex}=14.03 \mathrm{GPa}, \mathrm{Ey}=15.8 \mathrm{GPa}$ and $\mathrm{Ez}=32.6 \mathrm{GPa}$, which is anisotropic. The structure in $\mathrm{z}$-axis represents the stiffer direction compared with $\mathrm{x}$ and $\mathrm{y}$ directions. The Young modulus in $\mathrm{z}-$ axis (Ez) represents values of around two times higher than the other axes (Ex and Ey), which is in good agreement with results of Speziale et al. (2008), which showed Ez to be equal to 1.6 times Ex. The crystallography form of Ettringite composed of $[\mathrm{Al}(\mathrm{OH}) 6]^{3-}$ can be the reason of its stiffer structure in c-axis ( $\mathrm{z}$ direction).

A comparison between the Portlandite and Ettringite results shows that the Young modulus of Ettringite is around $60 \%$ lower than that of the Portlandite, which indicates that Ettringite stiffness is around 2.5 times lower than that of Portlandite. The low stiffness of Ettringite is due to a structure composed mostly of water molecules, which decrease its density to $1.79 \mathrm{~g} . \mathrm{cm}^{3}$. It is well known that 
density is one of the paramount factors affecting the mechanical properties of materials. The water in Ettringite structure reduces the cohesion between particles and shields the interatomic forces.

The Portlandite investigated in this study supports the use of COMPASS force field. However, this method of simulation in not applicable for Ettringite structure. COMPASS force field is not designed for cementitious materials, so the low Young modulus of Ettringite can be attributed to the precision and accuracy of COMPASS force field used for Portlandite compared to the Universal force field used for Ettringite.

\subsection{Summary}

The objective of the work detailed in this chapter has been to determine the elastic modulus of Portlandite and Ettringite. The molecular dynamic simulation was carried out with two force fields: COMPASS and Universal. Compared to the experimental results shown in literature, the elastic properties of Portlandite are slightly different. However, good agreement was noticed between the simulation results of this study and others computational methods.

Like Portlandite, the results of Ettringite also show differences compared to the experimental results and are in close agreement with the results of other simulation methods (e.g. Density Function Theory (DFT)). In addition, the Young modulus of Ettringite shows anisotropy in which the z-direction value is twice that of the two other directions. The c-axis (z) represents the stiffer axis due to the structure of Ettringite that runs in $\mathrm{z}-$ axis with $[\mathrm{Al}(\mathrm{OH}) 6]^{3-}$.

In comparing results of Portlandite and Ettringite, a higher elastic modulus of Portlandite was noticed, which is related to the high water molecules in the structure of Ettringite compared to Portlandite. 


\section{Chapter 6 Elastic Properties of analog minerals}

\subsection{Introductin}

Although there are 30 mineral crystals with chemical composition similar to C-S-H created from cement paste, there are some differences in atomic arrangement, $\mathrm{Ca} / \mathrm{Si}$ ratio and the number of $\mathrm{OH}$ and $\mathrm{H}_{2} \mathrm{O}$ (Taylor, 1997). According to literature, Tobermorite and Jennite are the minerals with the

most similarities to C-S-H. There are three types of Tobermorite: Tobermorite $9 \AA$, Tobermorite $11 \AA$ and Tobermorite $14 \AA$, which differ by their degree of hydration. Jennite is also similar to C-S-H structure at later ages of hydration, with a $\mathrm{Ca} / \mathrm{Si}$ ratio of 1.5 (as explained in chapter 1, section 1.11.4). The purpose of this chapter is to determine the elastic modulus of analog C-S-H minerals such as the Tobermorite family and Jennite with the use of molecular dynamic simulation by COMPASS force field. It also compares Young modulus results from this study with previous experimental and theoretical results in literature. This final purpose of this chapter is to determine the Tobermorite and Jennite structure closest to C-S-H.

\subsection{State of art of elastic properties of Tobermorite and Jennite}

\subsubsection{Tobermorite $14 \mathrm{~A}$}

Oh et al. (2012) performed an experimental study to determine the bulk modulus of Tobermorite $14 \AA$. They measured the unit cell volume change by increasing the pressure to $4.8 \mathrm{GPa}$ using high-pressure synchrotron X-ray diffraction under hydrostatic condition. The Tobermorite sample was finely ground and mixed with a pressure transmitting liquid (methanol/ethanol) to generate hydrostatic pressure. The X-ray diffraction pattern was collected at different pressures. As the applied hydrostatic 
pressure was increased, the unit cell of Tobermorite $14 \AA$ deformed, leading to a change in the lattice parameters. By analyzing X-ray diffraction patterns at different pressures, the lattice parameter was determined based on the positions of diffraction peaks. The volume of the lattice was calculated from the lattice parameters. Only the bulk modulus of Tobermorite $14 \mathrm{~A}$ was determined to be $\mathrm{K}_{0}=47 \mathrm{GPa}$. They also found that the bulk modulus of Tobermorite 14A depends on lattice parameter $\mathrm{c}$, which is related to the interlayer spacing composed of dreierketten silicate chains, interlayer $\mathrm{Ca}$, and water molecules (Oh et al., 2012).

Among the different theoretical approaches, molecular dynamics and ab-initio methods were the most common techniques used in literature to determine the elastic properties of Tobermorite 14A. The theoretical results show some differences, which depend on simulation method, number of cells and force field used.

\subsubsection{Tobermorite $11 \AA$}

The Tobermorite $14 \AA$ was heated at a temperature of $125^{\circ} \mathrm{C}$ for 24 hours, which led to the formation of Tobermorite $11 \AA$ with a peak diffraction around $11.3 \AA$, which also indicates reduced interlayer thickness. Using the same experimental method described in section 5.2.1 (chapter 5), Moon (2013) calculated the bulk modulus of Tobermorite $11 \AA$ to be $\mathrm{Ko}=62$ (4) GPa where the value between parentheses represents the standard deviation.

\subsubsection{Tobermorite 9A}

Moon (2013) performed an experimental study on Tobermorite $9 \AA$ to determine its bulk modulus. The Tobermorite $9 \AA$ was prepared by heating Tobermorite $14 \AA$ at $400^{\circ} \mathrm{C}$. The bulk modulus of Tobermorite $9 \AA$ was determined by measuring the unit cell volume change by increasing the pressure up to $8.5 \mathrm{GPa}$ using high-pressure synchrotron X-ray diffraction under a hydrostatic condition, using the same procedure described for Tobermorite $14 \AA$. Moon (2013) calculated the bulk modulus of Tobermorite $9 \AA$ to be $\mathrm{Ko}=82(4) \mathrm{GPa}$. 


\subsubsection{Jennite}

Jennite is the other mineral that is believed to be analog to $\mathrm{C}-\mathrm{S}-\mathrm{H}$ at a late stage of hydration. The structure of Jennite was solved by Bonnacorsi et al. (2004). Moon et al. (2015) performed an experimental study on Jennite to determine its bulk modulus, and also used the first principal method to determine its other elastic properties. Using high-pressure synchrotron X-ray diffraction under hydrostatic, Moon et al. (2015) found that the bulk modulus of Jennite is $\mathrm{K}_{0}=64$ (2) GPa, where the value between parentheses represents the standard deviation. From the first principal method, they found bulk, shear and Young modulus were $62 \mathrm{GPa}, 28 \mathrm{GPa}$ and $72.9 \mathrm{GPa}$, respectively, with a Poisson ratio of 0.3 .

\subsection{Computational method}

\subsubsection{Crystal parameters}

As the first step in the molecular simulation, the crystal structure was built by entering the data of the space group (which represents the symmetry of the structure), lattice type (triclinic or monoclinic) and lattice parameters $(\mathrm{a}, \mathrm{b}, \mathrm{c}, \alpha, \beta$, and $\gamma$ ) of Tobermorite 9A, 11A, 14A and Jennite. Those data (summarized in Table 11) were resolved experimentally by Merlino et al. (1999 and 2001) and Bonaccorsi et al. (2004 and 2005). 
Table 11: Lattice parameters of different models of Tobermorite and Jennite

\begin{tabular}{ccccc}
\hline $\begin{array}{c}\text { Lattice } \\
\text { Parameters }\end{array}$ & Tobermorite 9A & $\begin{array}{c}\text { Tobermorite } \\
\mathbf{1 1 A}\end{array}$ & $\begin{array}{c}\text { Tobermorite } \\
\mathbf{1 4 A}\end{array}$ & Jennite \\
\hline Space group & C1 & BM & B11b & P1 \\
Lattice type & Triclinic & Monoclinic & Monoclinic & Triclinic \\
$\mathbf{a}$ & 11.156 & 6.735 & 6.735 & 10.576 \\
$\mathbf{b}$ & 7.303 & 7.385 & 7.425 & 7.265 \\
$\mathbf{c}$ & 9.566 & 22.487 & 27.987 & 10.931 \\
$\boldsymbol{\alpha}$ & 101.080 & 90.000 & 90.000 & 101.300 \\
$\boldsymbol{\beta}$ & 92.830 & 90.000 & 90.000 & 96.980 \\
$\boldsymbol{\gamma}$ & 89.980 & 123.250 & 123.250 & 109.650 \\
Ref & {$[\mathrm{a}]$} & {$[\mathrm{b}]$} & {$[\mathrm{c}]$} & {$[\mathrm{d}]$}
\end{tabular}

[a]: (Merlino et al., 1999)

[b]: (Merlino et al., 2001)

[c]: (Bonaccorsi et al., 2005)

[d]: (Bonaccorsi et al., 2004)

As shown in Table 11, Tobermorite 9A, 11A, 14A and Jennite represent different space groups. Although Tobermorite 9A and Jennite, and Tobermorite 11A and 14A have the same triclinic or monoclinic lattice types, respectively, these similarities in crystal family (lattice type) have not been reported in literature, offering no preliminary indication or conclusion. After building the crystal structure using the data in Table 11, the different types of atoms were added to the crystal unit cell, which represents an imaginary parallelepiped of a repeating pattern.

\subsubsection{Geometry optimization parameters}

When the final structures of Tobermorite 9A, 11A, 14A and Jennite are built with atoms, a geometry optimization is usually needed to bring the geometry to a stable state. The process of geometry optimization or minimization is an iterative process where the energy structure is brought to its minimum energy and the force between atoms is zero. More information about the geometry optimization in the molecular simulation is provided in chapter 2 , section 2.12 . Table 12 shows the 
different parameters used to optimize the lattice structure including force field, quality, summation method and cut-off distance.

Table 12: Geometry minimization parameters

\begin{tabular}{cc}
\hline \hline \multicolumn{2}{c}{ Geometry minimization parameters } \\
\hline \hline Force field & COMPASS \\
Quality & Ultrafine \\
Summation Method & Ewald \\
Repulsive cutoff & $12.5 \mathrm{~A}$
\end{tabular}

The appropriate force field is very important in molecular dynamic simulation, since it determines the potential energy function of the structure, and adjusts atom position to reduce energy expression by using different algorithms to locate the minimum energy. In this study, COMPASS force field was applicable for all the analog C-S-H minerals.

The calculation of minimum energy was controlled by the quality setting. Ultrafine quality was used because it offers the most precise energy calculation. For instance, energy accuracy is set to $2 \times 10^{-5}$ $\mathrm{kcal} / \mathrm{mol}$ for ultrafine quality, while it is set to $0.02 \mathrm{kcal} / \mathrm{mol}$ for coarse quality. The Ewald-type summation method was used in this study because it has been reported in literature as the best option for the calculation of non-bond energy in periodic lattice. A cut-off distance of $12.5 \mathrm{~A}$ was used to reduce the number of interactions and to cut the contribution of van der Waals energy for Ewald summation

\subsubsection{Dynamic simulations parameters}

The dynamic simulation was performed after optimizing the structure. The crystal system was simulated as it occurs in the natural environment, where it may be under external pressure and varied temperatures. Table 13 shows the different parameters used in the molecular dynamic simulations of the present study 
Table 13: MD simulation parameters

\begin{tabular}{cc}
\hline \multicolumn{2}{c}{ Dynamic Simulation parameters } \\
\hline Ensemble & NPT \\
Temperature & $298 \mathrm{~K}\left(25^{\circ} \mathrm{C}\right)$ \\
Pressure $(\mathrm{GPa})$ & 0.0001 \\
Time step (fs) & 0.5 \\
Total simulation (ps) & $200-500$ \\
Thermostat & Nose \\
Barostat & Anderson
\end{tabular}

Different methods can be used to control temperature and pressure. For all the simulations in this study, an NPT ensemble was used (where $\mathrm{N}$ is number of particles, $\mathrm{P}$ is pressure and $\mathrm{T}$ is temperature), which remained fixed where the unit cell was allowed to change. (Other ensembles such as NVE and NVT can be also used.) Thermostat and Barostat are temperature and pressure control methods used to regulate temperature and pressure, respectively. One of the most important parameters is the time step integration of Newton equation, where higher time steps can lead to errors in the simulation. Additional information about ensembles is provided in chapter 2, section 2.19.

\subsection{Results of molecular simulation}

\subsubsection{Elastic properties of Tobermorite and Jennite}

Tables 15 and 16 show the elastic modulus of Tobermorite $9 \AA$, $11 \AA$, $14 \AA$ and Jennite resulting from our simulation using COMPASS force field. Other experimental and theoretical results from literature are also presented in Tables 14 and 15 for comparison. 
Table 14: Elastic properties of Tobermorite $9 \AA$ and Tobermorite $11 \AA ̊$ (Appendix VII, VIII)

\begin{tabular}{|c|c|c|}
\hline Mineral & Tobermorite 9A & Tobermorite 11Ă \\
\hline$\overline{K_{\mathbf{o}}(\mathbf{G P a})}$ & 87.7 & 48.3 \\
\hline G (GPa) & 34.7 & 25.7 \\
\hline E (GPa) & 92 & 65.5 \\
\hline $\mathbf{v}$ & 0.34 & 0.27 \\
\hline $\begin{aligned} \text { Ref } & : \text { Ko }(\mathbf{G P a}) \\
& : \mathbf{G}(\mathbf{G P a}) \\
& : \mathrm{E}(\mathbf{G P a})\end{aligned}$ & 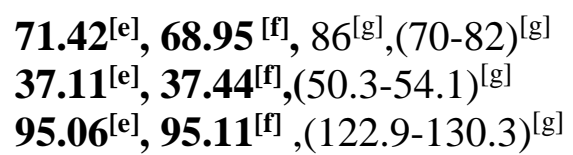 & $\begin{array}{l}66.65^{[\mathrm{e}]}, 73.57^{[\mathrm{f}]}, 68^{[\mathrm{g}]}, 67^{[\mathrm{g}]} \\
32.03^{[\mathrm{e}]}, \mathbf{2 9 . 1 9} \\
82.82^{[\mathrm{e}]}, \mathbf{7 7 . 3 4 ^ { [ \mathrm { f } ] }}, 32^{[\mathrm{g}]}, 83.1^{[\mathrm{g}]}\end{array}$ \\
\hline
\end{tabular}

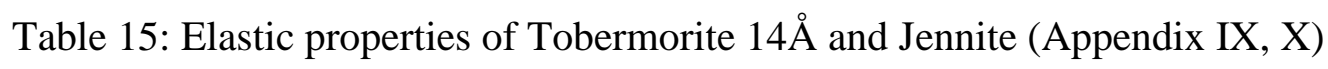

\begin{tabular}{|c|c|c|}
\hline Mineral & Tobermorite 14Å & Jennite \\
\hline$\overline{\mathbf{K}_{\mathbf{0}}(\mathbf{G P a})}$ & 40.9 & 73.3 \\
\hline G (GPa) & 16.7 & 23.9 \\
\hline E (GPa) & 44.1 & 64.7 \\
\hline $\mathbf{v}$ & 0.32 & 0.35 \\
\hline $\begin{array}{l}\text { Ref : Ko }(\mathbf{G P a}) \\
\quad: \mathbf{G}(\mathbf{G P a}) \\
\quad: \mathrm{E}(\mathbf{G P a})\end{array}$ & 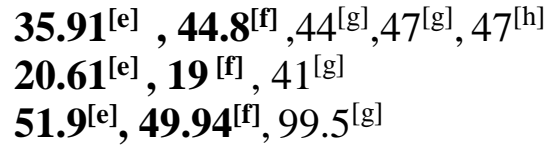 & $\begin{array}{l}31.83^{[\mathrm{e}]}, 40.2^{[\mathrm{f}]}, \mathbf{6} 4^{[\mathrm{ij}]} \mathbf{6 2}^{[\mathrm{i}]} \\
21.96^{[\mathrm{e}]}, 22.10^{\mathrm{ff}]}, \mathbf{2 8}^{[\mathrm{i}]} \\
53.55^{[\mathrm{e}]}, \quad 56.03^{[\mathrm{f}]}, \mathbf{7 2 . 9 ^ { [ i ] }}\end{array}$ \\
\hline
\end{tabular}

Ko: Bulk modulus

G: shear modulus

E: Young modulus

v: Poisson ratio

[e]: (Shahsavari et al., 2009)

[f]: (Manzano et al., 2007)

$[\mathrm{g}]:$ (Moon, 2013) (experimental and computation)

[h]: (Oh et al., 2012)

[i] : (Moon 2015) (experimental and computation)

As seen from Table 14, the Young modulus of Tobermorite 9A in this study was 92GPa, which is in good agreement with the results of Shahsavari et al. (2009) and Manzano et al. (2007); they showed a modulus of around $95 \mathrm{GPa}$ using other molecular dynamic simulation method. However, the experimental method performed by Moon (2013) showed slightly higher results that ranged between 122.9 GPa and 130.3 GPa.

Although the Young modulus of Tobermorite 11A was estimated to be equal 65.5GPa, the simulation in this study showed it to be slightly lower than the theoretical and experimental results attained by 
Shahsavari et al. (2009) (82.82 GPa) and Moon (2013) (83.1 GPa). Manzano et al. (2007) found a modulus of $77.34 \mathrm{GPa}$, which is only $11 \%$ higher than our results. In addition, the difference between the experimental values found by Moon (2013) and the Young modulus of our simulation do not exceed $21 \%$, which is acceptable compared to other simulation methods.

The Young modulus of Tobermorite 14A, resulting from our molecular dynamic simulation with COMPASS force field, was estimated to be $44.1 \mathrm{GPa}$, which is close to the modulus found by Manzano et al. (2007) (49.94GPa) using different molecular dynamic method. Although the experimental value showed by Moon (2007) was significantly higher than our simulation result, it was also higher than the other numerical simulation results completed by Shahsavari et al. (2009) and Manzano et al., (2007). This indicates that all numerical simultion methods underestimate the Young modulus of Tobermorite $14 \mathrm{~A}$.

The elastic properties of Jennite displayed in Table 16 show the Young modulus estimated in this study to be $64.7 \mathrm{GPa}$. Althougth this value is slightly higher than those expected by the ab-intio numerical method completed by Shahsavari et al. (2009) (53.55 GPa) and Manzano et al. (2007) (56.03 GPa), it is closer to the result measured by Moon (2013). The difference between the Young modulus found by COMPASS force field (in this study) and the experimental value (Moon result) is around $11 \%$, which is two times lower than the difference between the other theoretical results completed by the ab-initio method and the experimental Young modulus found by Moon (2013).

\subsubsection{Relation between Young modulus and interlayer spacing}

Figure 50 present the variation of elastic modulus results as a function of the interlayer spacing 


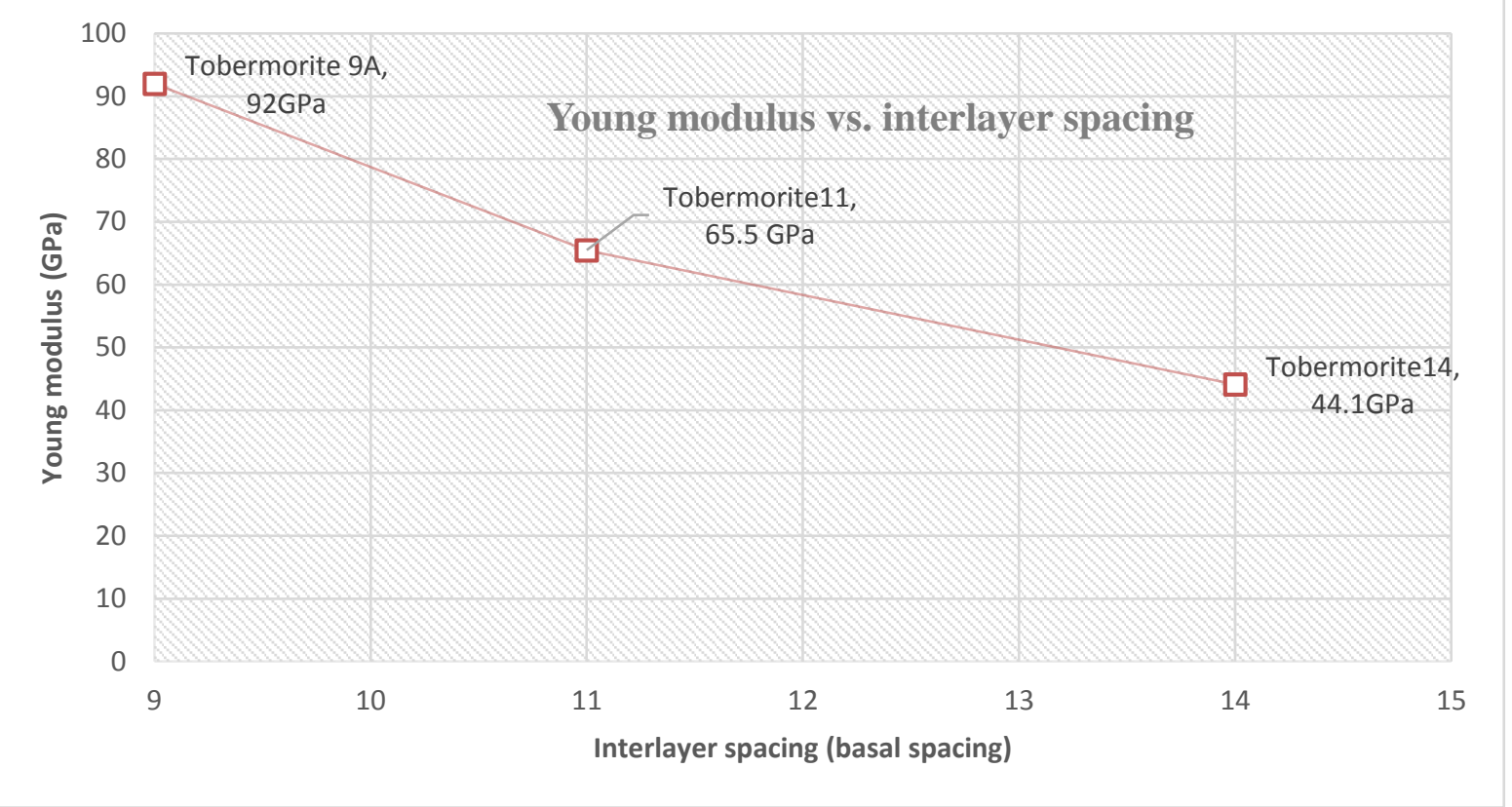

Figure 50 : Relationship between elastic modulus and interlayer spacing of Tobermorite family

Figure 50 shows that the interlayer spacing considerably affects the elastic modulus of the Tobermorite family (9A, 11A and 14A). For instance, Tobermorite 9A (characterized by its thinner interlayer spacing) has a higher elastic modulus, whereas 14A (with its larger interlayer spacing) is the softest Tobermorite mineral. It is clear from Figure 50 that the increase in interlayer spacing causes the elastic modulus to decrease.

\subsubsection{Relation between Young modulus and C/S, density and water}

Table 16 presents some important parameters of Tobermorite 9A, 11A, 14A and Jennite, such as C/S ratio, water to calcium ratio $(\mathrm{W} / \mathrm{Ca})$, water to silica ratio $(\mathrm{W} / \mathrm{Si})$ and density. $\mathrm{C} / \mathrm{S}, \mathrm{W} / \mathrm{Ca}, \mathrm{W} / \mathrm{Si}$ were determined from different chemical formulas, whereas density values were extracted from the materials studio software before bringing the structure to its relaxed state, and before running the molecular dynamic (MD) simulation. The relationships between elastic modulus and C/S, density, W/Ca and W/Si ratios are presented in Figures 51, 52, 53 and 54, respectively.

Table 16: Properties of different types of Tobermorite and Jennite (C/S, density, W/Ca and W/Si and elastic properties) 


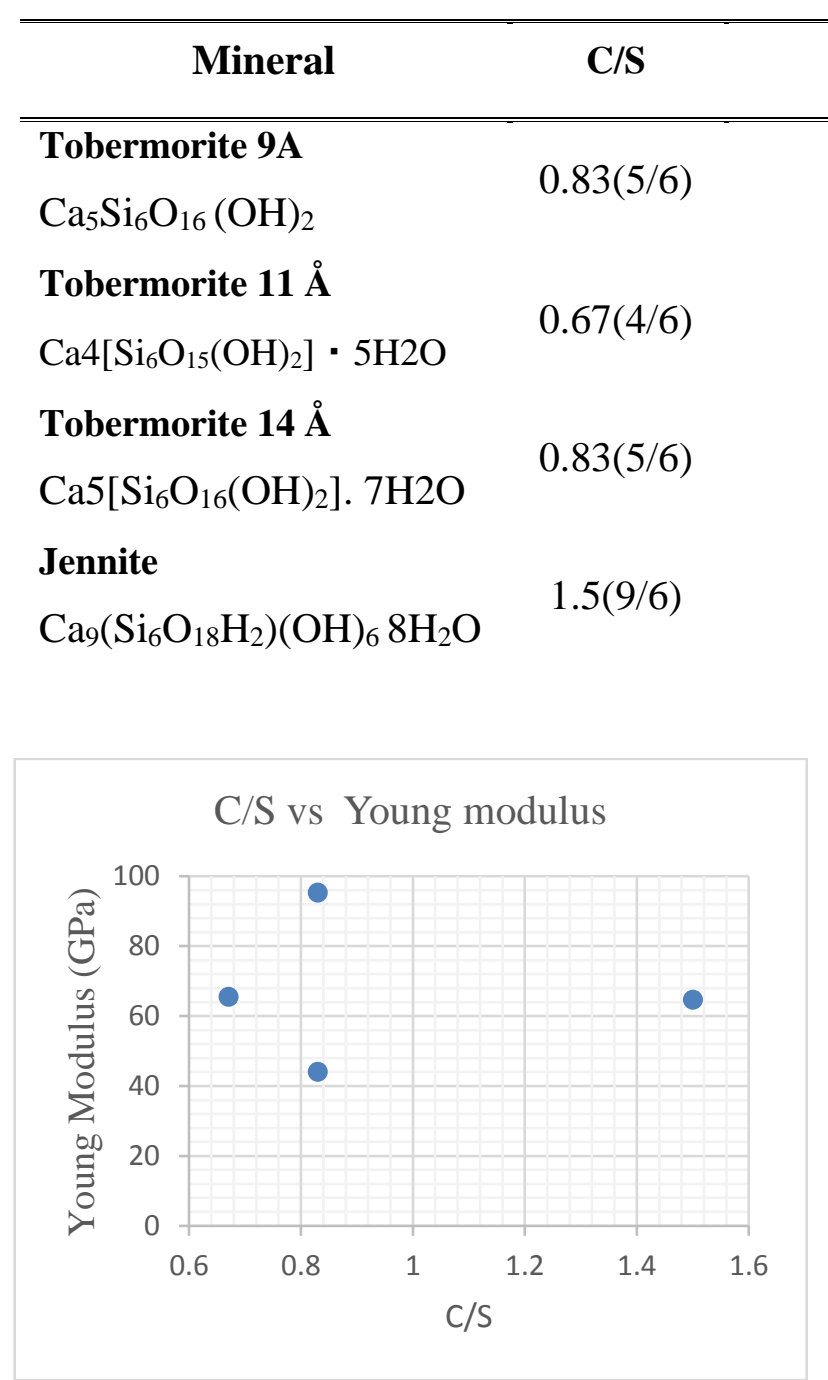

Figure 51: Young Modulus vs. C/S ratio

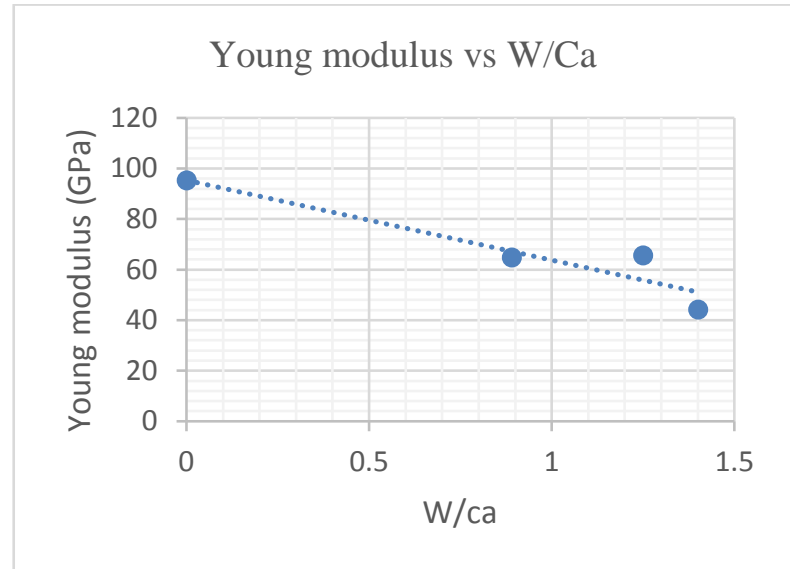

Figure 53: Young modulus vs. W/Ca ratio
Density W/Ca W/Si $\quad$ E

$\begin{array}{lll}2.681 & 0 & 0\end{array}$

95.23

$\begin{array}{lll}2.453 & 1.25 & 0.83\end{array}$

65.5

2.245

1.4

1.16

44.1

2.32

0.89

1.33

64.7

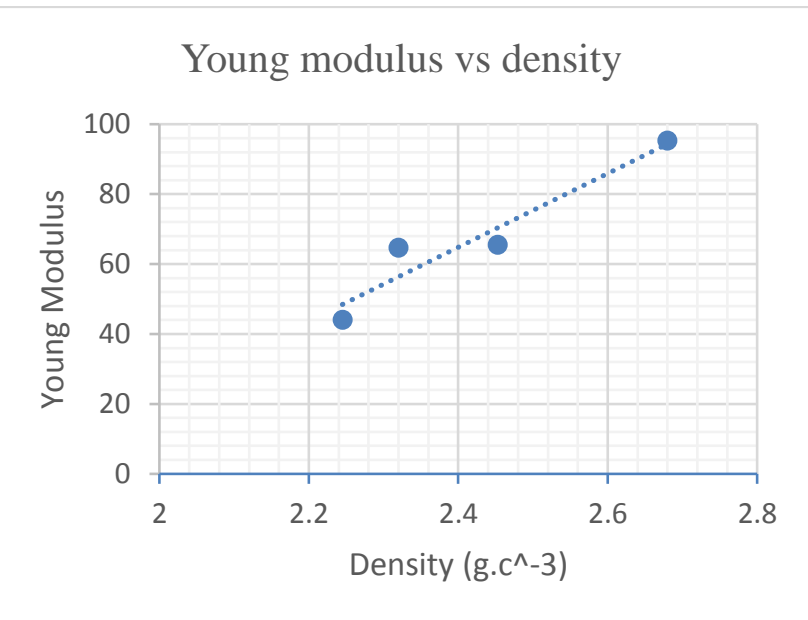

Figure 52 : Young modulus vs. density

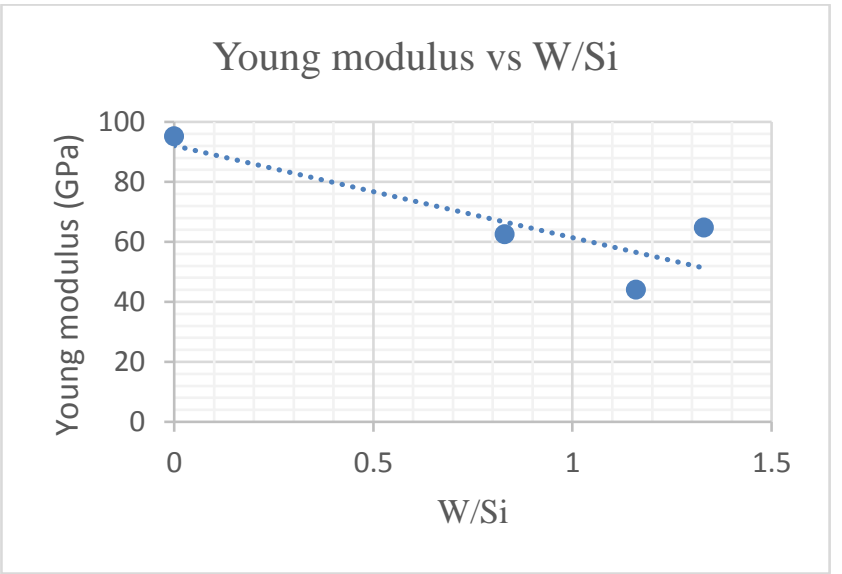

Figure 54: Young modulus vs. W/Si ratio

As shown in Figure 51, there is no clear relationship between C/S ratio and Young modulus results of analog C-S-H minerals. For example, while Tobermorite $9 \AA$ and $14 \AA$ had the same C/S ratio of 
0.83, they also show diverse elastic modulus. In addition, Tobermorite $11 \AA$ and Jennite have close elastic modulus results, although they present large differences in $\mathrm{C} / \mathrm{S}$ ratio, with values of 0.67 and 1.5, respectively. Figure 52, however, indicates a clear relationship between density and the difference in elastic modulus of the Tobermorite family and Jennite. Tobermorite 9A, which showed the highest elastic modulus, has the higher density. Tobermorite 14A, with the lowest density, is characterized by its lower elastic modulus.

Water has a direct impact on elastic modulus results. Figures 53 and 54 show that as interlayer water increased by $\mathrm{Ca}$ or $\mathrm{Si}$, elastic modulus decreased. The Tobermorite $14 \mathrm{~A}$ with the highest W/Ca and W/Si ratios also had the lowest modulus of elasticity. This can be explained by the fact that, as water in the interlayer spacing increased, the silicate chains became more distant from each other and reduced the interlayer interaction between atoms in the molecular dynamic simulation, as well as the cohesiveness of the structure. In addition, the reduced interlayer spacing of Tobermorite 9A and 11A creates a denser structure than in $14 \mathrm{~A}$, which is also related to the quantity of water in the interlayer spacing and hence the elastic modulus.

\subsection{Discussion of results}

Molecular dynamics simulations using COMPASS force field were performed to determine the elastic properties of analog $\mathrm{C}-\mathrm{S}-\mathrm{H}$ minerals (Tobermorite and Jennite). The values resulting from those simulations had similarities and differences to the results of methods previously used in literature. The greatest differences were evident with the ab-initio method, which can be explained by the difference in the number of cells used in each dynamic simulation method and in the force field used. This explanation is in line with conclusions made by $\mathrm{Wu}$ et al. (2011), who investigated the combination of different simulation cell sizes and force fields. These authors confirmed that simulation is largely dependent on force field and cell size. Another reason may be the position of hydrogen in the water molecule. Since no information was provided about hydrogen coordinates in 
the other studies, its position can be very different from one simulation to another, which may also influence results.

The elastic modulus of Tobermorite $9 \AA$ was higher than in $11 \AA$ and $14 \AA$. The dissimilarity in elastic modulus between the different types of Tobermorite can be explained by the difference in interlayer thickness, in which Tobermorite 9A has the thinner interlayer, and 14A has the larger interlayer. This explanation agrees with conclusions made by Oh et al. (2012), who used X-ray diffraction to determine the bulk modulus of Tobermorite $14 \AA$. They stated that modulus values of Tobermorite $14 \AA$ are controlled by the incompressibility of the lattice parameter c (z-axis), which is parallel to the interlayer spacing. The increase in the interlayer spacing reduces the cohesion between silicates chain, which leads to the decrease in elastic modulus.

Based on the density values of Tobermorite cells $(9 \AA, 11 \AA$, and $14 \AA)$ and Jennite, Tobermorite 9A structure has the highest density with the highest elastic modulus, and Tobermorite 14A has the lowest density with the lowest elastic modulus. The clear relationship between elastic modulus and density can be related to the packing of particles. As density increases, particles get closer to each other, which also increases elastic modulus. This suggestion agrees with the results of Constantinides and Ulm (2007), which showed a correlation between low density and low stiffness.

As shown in the correlation between elastic modulus and $\mathrm{W} / \mathrm{Ca}$, and $\mathrm{W} / \mathrm{Si}$, when water increases, Young modulus decreases. This conclusion is in line with most of the results presented in literature. According to Shahsavari et al. (2009), when excess water molecules enter the interlayer, the interlayer space increases and Coulombic interactions reduce. This can cause reduction of the elastic properties in the interlayer direction as W/Ca or W/Si increases.

Another important point concerns the results of Young modulus estimated in the three axes (Appendices VII, VIII, IX and X). With Young modulus results of Ex=86.74GPa, Ey=137.26GPa, and $\mathrm{Ez}=110.4 \mathrm{GPa}$, Tobermorite $9 \mathrm{~A}$, which did not contain any water molecules, showed anisotropic properties due to its layered structure. The highest value was in the y-axis (b) direction, parallel to the silicate chain, which means that the silicate tetrahedral $\mathrm{SiO}_{4}$ had more of an effect on 
determination of Young modulus. This conclusion agrees with the results found by Tunega end Zaoui (2010), which showed that the stiffest Young modulus is on the $y$-axis (b) direction, and is related to the silicate chain that runs in that direction.

Tobermorite 11A, which contained some water molecules in the interlayer, presented elastic modulus of $\mathrm{Ex}=65.19 \mathrm{GPa}, \mathrm{Ey}=94.62 \mathrm{GPa}$ and $\mathrm{Ez}=67.19 \mathrm{GPa}$ (Appendix VIII) in the three axes. These results also confirm an anisotropy, in which the elastic modulus in $\mathrm{x}$ and $\mathrm{y}$-direction were similar (Ex Ez), whereas the strongest Young modulus was on the y-axis parallel to the silicate chain. These findings also confirm the Tunega end Zaoui (2010) results mentioned above.

As mentioned in Chapter 1, section 1.10, the Young modulus of C-S-H (LD and HD) measured experimentally by nano-indentation, ranges between 20 and 30GPa, as noted by Canstantinides et al. (2013). However, in this study, the Young modulus resuls of Tobermorite 9A, 11A, 14A and Jennite were found to be equal to $95.23 \mathrm{GPa}, 65.5 \mathrm{GPa}, 44.1 \mathrm{GPa}$ and $64.7 \mathrm{GPa}$, respectively. This shows that the results of the analog C-S-H minerals present higher values than the experimental Young modulus of C-S-H measured by nano-intendation. The greater differences between the results of our simulation and the Young modulus of C-S-H measured experimentally can be explained by two reasons: 1) the infinite silica chain length used in our molecular simulation, or 2) the fact that the porosity of different analogue C-S-H minerals was not accounted for. These suggestions are covered in more detail in Chapter 7.

\subsection{Summary}

The purpose of this chapter was to evaluate the use of molecular dynamic simulation by COMPASS force field to estimate the elastic modulus of different types of Tobermorite (9A, 11A and 14A) and Jennite, which are assumed to have some similarities with the structure of C-S-H. Parameters that affect the elastic modulus variations were also determined.

The results achieved in this study present some dissimilarities with the experimental measurements, especially for Tobermorite 9A and 14A. However, most of the Young modulus results line up with 
the theoretical values reported in literature. In addition, the Young modulus of Tobermorite 11A and Jennite present only small differences (around 11\%) compared to the experimental measurements outlined in literature.

Based on the results of this study, the parameters that considerably affect the elastic properties of analog C-S-H minerals are: 1) interlayer spacing for different types of Tobermorite, 2) W/Ca and $\mathrm{W} / \mathrm{Si}$, and 3) density. For the first parameter (interlayer spacing), as spacing was reduced, elastic modulus increased and the structure became more compressible and less stiff. For the second parameter $(\mathrm{W} / \mathrm{Ca}$ and $\mathrm{W} / \mathrm{Si}$ ), excess water molecules negatively affected elastic modulus by increasing interlayer spacing and reducing the interaction force between layers. The third parameter (density) is related mostly to the packing of particles, in which elastic modulus increased when density increased.

Although all analog C-S-H minerals showed higher Young modulus compared to C-S-H, Tobermorite 9A presented the highest difference, with a value more than three times that of C-S-H. In addition, the comparison between C/S ratios of C-S-H (LD and HD), measured experimentally, (which ranged between 1.2 and 2.3, as discussed in chapter 1, section 1.7) and those of Tobermorite 9A (0.83), 11A (0.67), 14A (0.83) and Jennite (1.5), showed that Tobermorite 11A had the highest difference compared to C-S-H. Based on these results, it can be concluded that Tobermorite 14A and Jennite are the minerals closest to C-S-H. For that reason, only Tobermorite 14A and Jennite are considered in Chapter 7. 


\section{Chapter 7 C-S-H gel Elastic properties}

\subsection{Introduction}

In chapter 6 , the Young modulus values of Tobermorite $9 \AA, 11 \AA, 14 \AA$ and Jennite crystals were remarkably higher than those in the experimentally measured C-S-H gel. When the Young modulus values and C/S ratios of Tobermorite and Jennite are compared to those of C-S-H, Tobermorite 14A and Jennite are the closest minerals to C-S-H in terms of structure. However, with values of 44.1GPa for Tobermorite 14A and 64.7 GPa for Jennite, those analog C-S-H minerals have a Young modulus that is around two times higher than that of C-S-H, which ranges between 20 and 30GPa. The goal of this chapter is to investigate the use of molecular dynamic simulation with COMPASS force field to reduce the Young modulus of Tobermorite 14A and Jennite and make it closer to that of C-S-H. Two methods were considered:

The first method is based on conclusions made by Taylor (1997). In that study, the structure of C-SH showed similarities with Tobermorite and Jennite, with finite silicate chain following the rule of $3 \mathrm{n}-1$ (where $\mathrm{n}$ is an integer number). The structure of Tobermorite 14A and Jennite in our molecular simulation were modified to be finite silicate chain in which $n=1$ (dimer), rather than the infinite chain used in chapter 6.

In the second method, because the experimental elastic modulus of C-S-H covered in literature was measured in low and high density (LD and HD) states with porosity equal to $36 \%$ and $26 \%$, respectively, and since the porosity of different analog C-S-H minerals was not accounted for in our previous simulations, this important parameter was investigated for Tobermorite 14A and Jennite with infinite and finite silica chains. 
In addition, the results of this study and the experimental elastic modulus of $\mathrm{C}-\mathrm{S}-\mathrm{H}$ mentioned in literature were compared to verify the reliability of the proposed solutions

\subsection{State of art on finite silicate chain}

The omission of the bridging tetrahedral silicon in Jennite does not change the total charge of the unit cell, where one silicon atom has $+4 \mathrm{e}^{+}$and two atoms of oxygen $\left(-2 \mathrm{e}^{-}\right)$are omitted. In the case of Tobermorite, the removal of one silicon atom $\left(4 \mathrm{e}^{+}\right)$, one oxygen $\left(-2 \mathrm{e}^{-}\right)$and one hydroxyl $\left(-1 \mathrm{e}^{-}\right)$leads to a negatively charged system (Figure 55). Taylor (1997) suggests that a hydrogen atom be added to the oxygen atom to equilibrate the system.
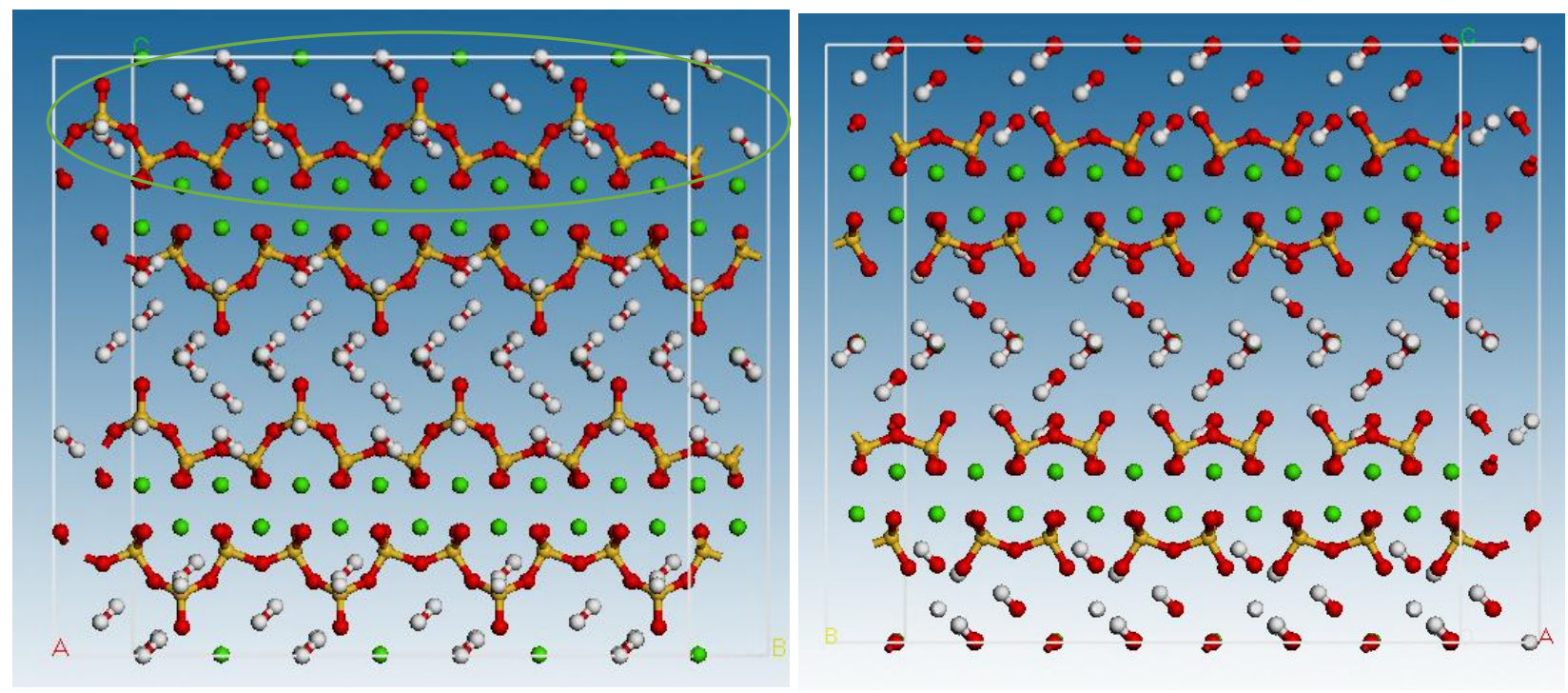

Figure 55: Tobermorite structure with infinite and finite chain (bridging silicate removed)

Because the silicate chain length has a direct relationship with $\mathrm{C} / \mathrm{S}$ ratio, removing the bridging silicate chain also modifies the $\mathrm{C} / \mathrm{S}$ ratios of Tobermorite 14A and Jennite. Omitting the bridging silicate tetrahedral is equivalent to removing $\mathrm{SiO}_{2}$ from the structure; the hydrogen in the silanol group is moved to the pair silicate tetrahedral. Consequently, the removal of $\mathrm{SiO}_{2}$ increases the $\mathrm{C} / \mathrm{S}$ ratio of Tobermorite 14A and Jennite. Indeed, according to the atomic model proposed by Taylor (1997) (chapter 2, section 2.7.4), the removal of the bridging increases C/S ratios of Tobermorite 14A and Jennite from 0.83 to 1.25 and from 1.5 to 2.25 , respectively. These new C/S ratios of Tobermorite 14A and Jennite with modified chain are in the range of $\mathrm{C} / \mathrm{S}$ ratios of $\mathrm{C}-\mathrm{S}-\mathrm{H}$ measured experimentally 
to be between 1.2 and 2.3, which suggests that the molecular dynamic simulation of modified structures of Tobermorite 14A and Jennite can result in an elastic modulus closer to that of C-S-H. Limited studies have been conducted into the simulation of defect structures of Tobermorite and Jennite, with only the ab-initio method used in molecular simulation. For instance, Manzano et al. (2007) completed a molecular simulation by ab-initio method on modified Tobermorite 14A and Jennite structures. They found the Young modulus to be equal to 35.6 and $43.7 \mathrm{GPa}$ for Tobermorite 14A and Jennite, respectively, which is close to the Young modulus of C-S-H. No molecular dynamic simulation has been done using COMPASS force field to determine the elastic properties of Tobermorite 14A and Jennite with modified structure.

In this study, the defect Tobermorite $14 \mathrm{~A}$ and Jennite are called $\mathrm{T} 2$ and $\mathrm{J} 2$, respectively, indicating a silicate chain without a bridging silicate.

\subsection{Computational method of finite silicate chain of Tobermorite $14 \mathrm{~A}$ and}

\section{Jennite}

\subsubsection{Geometry optimization parameters}

Geometry optimization of molecules brings the structure to its stable state. As a first step in all molecular dynamic simulations using COMPASS force field presented in this chapter, geometry optimization was run to bring the structure of modified Tobermorite 14A (T2) and Jennite (J2) to its relaxed state. Table 17 summarizes the parameters used to optimize the lattice structure, including force field used, quality, summation method and cut-off distance

Table 17: Geometry optimization parameters

\begin{tabular}{cc}
\hline \hline \multicolumn{2}{c}{ Geometry minimization parameters } \\
\hline \hline Force field & COMPASS \\
Quality & Ultrafine \\
Summation Method & Ewald \\
Repulsive cutoff & $6 \AA$
\end{tabular}




\subsubsection{Dynamic simulation parameters}

Table 18 shows the dynamic simulation parameters used in this study. Different methods and parameters can be used to run dynamic simulations (NVT, NVE and NPT). In this study, NPT method was used, in which $\mathrm{N}$ the number of atoms is, $\mathrm{P}$ is the pressure and $\mathrm{T}$ is the temperature. The choice of the total time of simulation and the time step is also important in determining whether the simulation has reached equilibrium before calculating average mechanical properties. In accordance with the reduced time of simulation targeted in this study, total simulation time was selected to be between 200 and 500 ps, and the time step equal to 0.5 fs.

Table 18: Dynamic simulation parameters

\begin{tabular}{cc}
\hline \hline \multicolumn{2}{c}{ Dynamic Simulation parameters } \\
\hline \hline Ensemble & NPT \\
Temperature & $298 \mathrm{~K}\left(25^{\circ} \mathrm{C}\right)$ \\
Pressure $(\mathrm{GPa})$ & 0.0001 \\
Time step (fs) & 0.5 \\
Total simulation time (ps) & $200-500$ \\
Thermostat & Nose \\
Barostat & Anderson
\end{tabular}

\subsubsection{Results of the molecular simulation}

7.3.3.1 Elastic properties of Tobermorite and Jennite with different silicate chains

The results of molecular simulation of the modified chain Tobermorite-like T2 and Jennite-like $\mathrm{J} 2$ by COMPASS force field are presented in Table 19. The results reported in chapter 6 about Tobermorite 14A $(\mathrm{T} \infty)$ and Jennite $(\mathrm{J} \infty)$ with infinite silica chain, are also presented here to show the effect of the removal of bridging (removing $\mathrm{SiO}_{2}$ ) on elastic properties. For comparison, results of the ab-initio method shown in literature are also provided in Table 19. 
Table 19: Elastic properties of Tobermorite and Jennite with different silicate chain lengths (Appendix IX, X, XI, XII)

\begin{tabular}{|c|c|c|c|c|}
\hline Mineral & $\mathbf{T} \infty$ & $\mathbf{T} 2$ & $\mathbf{J} \infty$ & $\mathbf{J} 2$ \\
\hline $\mathrm{K}_{\mathbf{o}}(\mathbf{G P a})$ & 40.9 & 42.9 & 73.3 & 71.4 \\
\hline G (GPa) & 16.7 & 12.8 & 23.9 & 16.8 \\
\hline $\mathbf{E}(\mathbf{G P a})$ & 44.1 & 34.93 & 64.7 & 46.7 \\
\hline $\mathbf{v}$ & 0.32 & 0.36 & 0.35 & 0.39 \\
\hline Density $\left(\mathrm{g} / \mathrm{cm}^{-3}\right)$ & 2.24 & 1.903 & 2.32 & 2.048 \\
\hline $\mathrm{C} / \mathrm{S}$ & 0.83 & 1.25 & 1.5 & 2.25 \\
\hline Ref : Ko (GPa) & $35.91^{[a]}, 44.80^{[b]}$ & $26^{[\mathrm{b}]}$ & $31.83^{[a]}, 40.2^{[b]}, 64^{[c]}$ & $23.9^{[b]}$ \\
\hline G (GPa) & $20.61^{[a]}, 19^{[b]}$ & $14^{[\mathrm{b}]}$ & $21.96^{[\mathrm{a}]}, 22.10^{[\mathrm{b}]}, 28^{[\mathrm{c}]}$ & $13.8^{[\mathrm{b}]}$ \\
\hline $\mathbf{E}(\mathbf{G P a})$ & $51.9^{[a]}, 49.94^{[b]}$ & $35.6^{[b]}$ & $53.55^{[\mathrm{a}]}, 56.0^{[\mathrm{b}]}, 72.9^{[\mathrm{c}]}$ & $34.7^{[b]}$ \\
\hline
\end{tabular}

[a]: (Shahsavari et al., 2009)

[b]: (Manzano et al., 2007)

[c] : (Moon et al., 2015) (experimental and computation)

[d] : (Rejmak et al., 2012)

Ko: Bulk modulus

G: shear modulus

E: young modulus

v: poison ratio
Tळ: Tobermorite with infinite silicate chain

T2: Tobermorite with dimer silicate chain $(n=1)$

$\mathrm{J} \infty$ : Jennite with infinite silicate chain

J2: Jennite with dimer silicate chain $(n=1)$

As shown in Table 19, the molecular simulation by COMPASS force field used in this study confirms that removing the bridging of silicate tetrahedrals considerably reduced the Young modulus of analog C-S-H minerals. For the infinite to finite silicate chain, the Young modulus of Tobermorite 14A and Jennite was reduced from 44.1 GPa to $34.93 \mathrm{GPa}$ and $64.7 \mathrm{GPa}$ to 46.7 , respectively. However, as mentioned above, the experimental Young modulus of $\mathrm{C}-\mathrm{S}-\mathrm{H}$ (LD and HD) measured by Constantinides (2013) ranged between 20 and $30 \mathrm{GPa}$. These results indicate that even after removing the bridging of silicate tetrahedrals, the Young modulus of Tobermorite T2 and Jennite $\mathrm{J} 2$ remained higher than that of C-S-H, but with fewer differences than the infinite silicate chain considered in chapter 5. In addition, the Young modulus of Tobermorite T2 resulting from the mechanical 
simulation by COMPASS force field showed a clear agreement with the results of Manzano et al. (2007), who used numerical simulation by GULP code. However, the Young modulus of Jennite J2 resulting from this study (46.7 GPa) shows some discrepancy compared to the value reported (34.7 GPa) from the ab-initio method used by Manzano et al. (2007).

Another notable result concerns the relationship between C/S ratio, silicate chain length and Young modulus. As illustrated in Table 19, as silicate chain length decreases, C/S ratios increases and Young modulus values decrease. For instance, when the silicate chain was modified from infinite to finite and the Young modulus of Tobermorite $(\mathrm{T} \infty)$ and Jennite $(\mathrm{J} \infty)$ were reduced from 44.1GPa to 34.93 $\mathrm{GPa}$ and $64.7 \mathrm{GPa}$ to $46.7 \mathrm{GPa}$, respectively, the $\mathrm{C} / \mathrm{S}$ ratios increased from 0.83 to 1.25 and 1.5 to 2.25, respectively. More correlations from the table are discussed below.

\subsubsection{Relation between Young modulus and chain length Tobermorite of Jennite}

Figure 56 shows the relationship between Young modulus results and silicate chain length of Tobermorite 14A and Jennite-like structures.
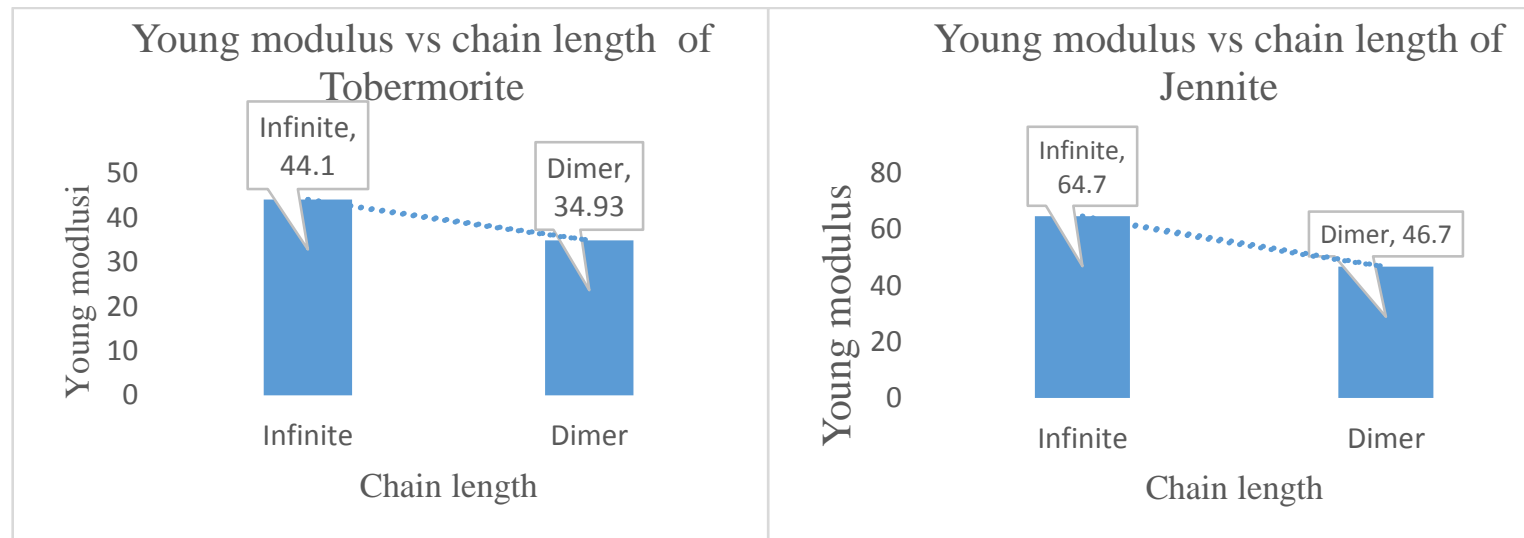

Figure 56: Relationship between Young modulus and silicate chain length of Tobermorite and Jennite

Figure 56 confirms the remark mentioned above in section 7.3.3.1; there is a clear relationship between Young modulus and chain length. With the removal of the bridging silicate tetrahedral (from infinite to finite structures), the Young modulus of Tobermorite and Jennite was reduced by $20 \%$ and $30 \%$, respectively. These results show that the decrease in the chain length has a considerable influence on Young modulus results, especially for Jennite-like structures 
7.3.3.3 Relation between Young modulus density and water

The relationship between Young modulus and density of Tobermorite and Jennite is presented in Figure 57

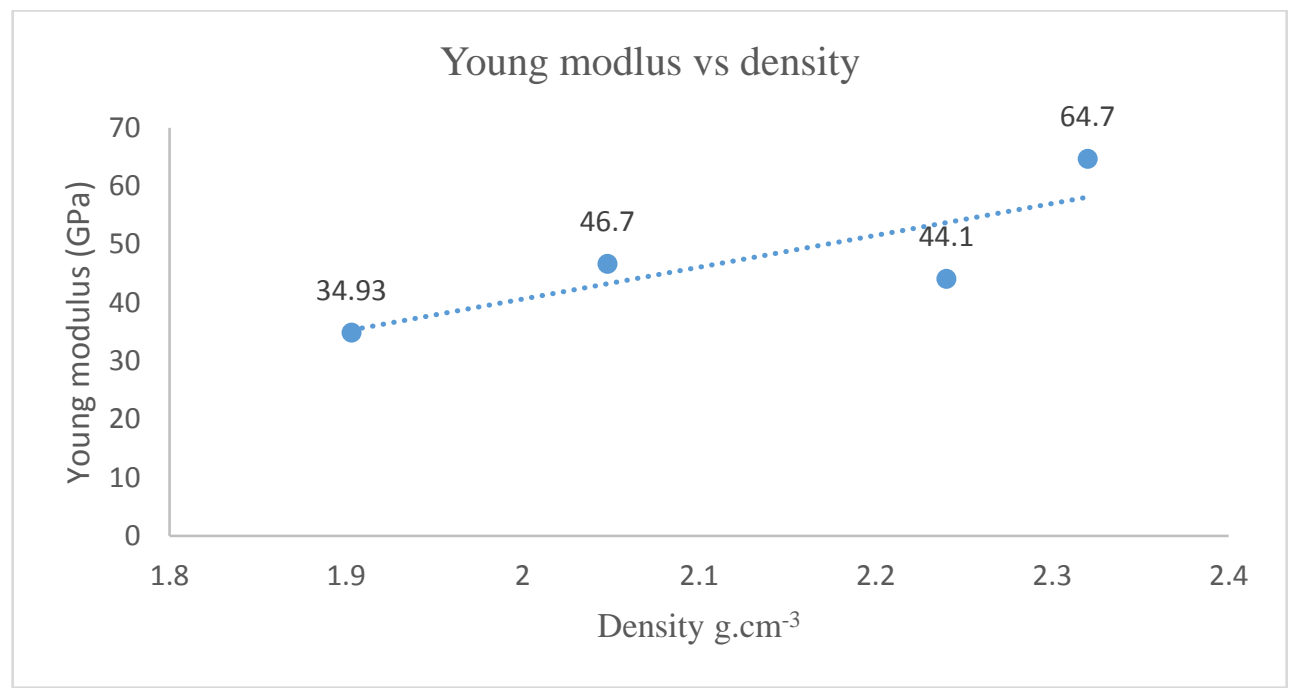

Figure 57: Relationship between Young modulus and density of Tobermorite, Tobermorite T2, Jennite and Jennite $\mathbf{J} 2$

Figure 57 shows that the decrease in Young modulus (related to the decrease in the chain length of Tobermorite and Jennite) causes a decrease in the density of crystals. For instance, Tobermorite 14A $(\mathrm{T} \infty)$ density that was $2.24 \mathrm{~g} . \mathrm{cm}^{-3}$ with infinite silicate chain, was reduced to $1.903 \mathrm{~g} . \mathrm{cm}^{-3}$ after removing the bridging (T2), which represents a decrement of around 15\%. The density of Jennite was reduced from $2.048 \mathrm{~g} . \mathrm{cm}^{-3}$ for $\mathrm{J} \infty$ to $2.32 \mathrm{~g} . \mathrm{cm}^{-3}$ for $\mathrm{J} 2$, which represents a $12 \%$ reduction. This result can be explained by the mass loss caused by the removal of the bridging silicate tetrahedral, which also reduced the total density of the crystal. The removal of the bridging silicate chain also caused the water to silicate ratio (W/Si) to increase in the lattice. Excess water can lead to a less dense structure and prevent interaction between atoms, resulting in a structure that is less stiff.

\subsubsection{Discussion}

In looking at the molecular dynamic simulation by COMPASS force field used in this study, the Young modulus of Tobermorite T2 was very close to the value found by Manzano et al. (2009). However, the Jennite $\mathrm{J} 2$ result was remarkably higher than that found by GULP code (Manzano et 
al., 2009). There is no clear reason for the similarity with T2 and the discrepancy with J2, especially considering that Manzano et al. used the same simulation by GULP code for both T2 and J2, and in this study, the same dynamic simulation by COMPASS force field was used for both samples.

Removing the silicate chain caused a remarkable reduction in the Young modulus of Tobermorite $14 \mathrm{~A}$ and Jennite. This conclusion is in line with the literature results. Due to atom mass loss, the removal of the bridging silicate tetrahedral was accompanied by a reduction in the density of Tobermorite 14A and Jennite, which can also reduce the stiffness and Young modulus of the analog C-S-H minerals. The decrements in Young modulus due to the removal of the bridging silicate chain show that the infinite chain provided stability to the structure, and that removal of bridging caused reduced cohesion between the layers of the structure.

Even after removing the bridging silicate tetrahedral, the Young modulus of Tobermorite 14A and Jennite showed higher values than those of C-S-H (LD and HD), which suggests that accounting for porosity for Tobermorite $\mathrm{T} 2$ and Jennite $\mathrm{J} 2$ after removing the bridging may lead to results closer to those of C-S-H. For this reason, porosity was accounted for in Tobermorite 14A and Jennite with infinite and finite silicate chains.

\subsection{The accounting of porosity for Tobermorite $14 \mathrm{~A}$ and Jennite}

\subsubsection{Mechanical properties of porous materials at microscale level}

Concrete is a heterogeneous material composed of random structures at different scales, from macroscopic to nanometer. Micromechanics offers a solution to this heterogeneity; in it, heterogeneous materials are separated into phases and the average of these phases is calculated. This procedure is called homogenization, a mathematical model that averages the properties of composite materials. Porosity is one of the most important properties of concrete. To account for it with micromechanics, two methods can be used: Mori-Tanaka and self-consistent formulas. Although they both rescale bulk and shear modulus using packing factor, the self-consistent method can better 
describe C-S-H, according to literature results (Constantinides and Ulm, 2007). For that reason, only the self-consistent method was used in this study.

Formulas to rescale the elastic modulus are as fellow:

$\frac{G_{N E W}}{G_{0}}=\frac{1}{2}-\frac{5}{4}(1-\theta)-\frac{3}{16} r_{0}(2+\theta)+$

$\frac{1}{16} \sqrt{144\left(1-r_{0}\right)-480 \theta+400 \theta^{2}+408 r_{0} \theta-120 r_{0} \theta^{2}+9 r_{o}^{2}(2+\theta)^{2}}$

$\frac{K_{n e w}}{K o}=\frac{\frac{4 \Theta G}{G_{O}}}{\frac{4 G}{G_{O}}+3(1-\theta) r_{o}}$

$\Theta$ : Packing factor $(\%)$

Ko , Go are the bulk and shear modulus (GPa)

$\mathrm{r}_{0}=\mathrm{Ko} / \mathrm{Go}$

Knew, Gnew: Bulk and shear modulus After applying the packing factor

After calculating bulk and shear modulus, elastic modulus can be calculated by using equations 3.25

and 3.26, explained previously in chapter 3, section 4.6.

\subsubsection{Elastic properties of C-S-H - low density (LD) and high density (HD) C-S-H}

Using a nano-indentation tool, Constantinides and Ulm (2006) revealed the existence of four frequency distributions of Young modulus (indentation modulus) that depend on water to cement (w/c) ratio. When they performed nano-indendation mapping on the surface area of cement paste (Figure 58), they found:

a) $\mathrm{M}$ between 0 and $13 \mathrm{GPa}$ represents the area with high porosity (macroporosity).

b) M between 13 and $26 \mathrm{GPa}$ represents the LD response.

c) M from 26 to $39 \mathrm{GPa}$ represents the third peaks that characterize high density (HD).

d) M> 39GPa represents the unreacted clinker phases and Portlandite.

Decreased w/c ratio leads to reduction of the low density peak, increase of the high density peak and a probable increase in the frequency of the clinker peak. 


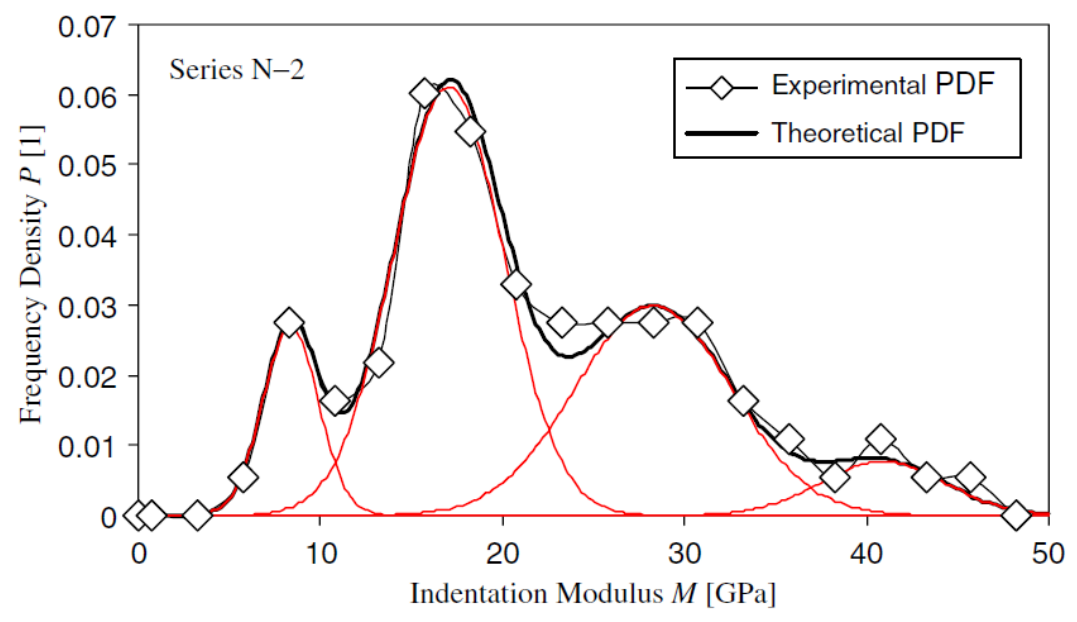

Figure 58: Frequency density as function of indentation modulus (10x10) indentation tests on a grid of spacing $10 \mu \mathrm{m}$ ) Constantinides and Ulm (2006), PDF; probability density function

\subsubsection{Elastic properties of analog C-S-H minerals accounting for porosity}

As explained above, the Young modulus results calculated in the previous section for T $\infty, \mathrm{T} 2$, J $\infty$ and J2 have higher elastic modulus compared to the experimental results of $\mathrm{C}-\mathrm{S}-\mathrm{H}$. In this section, porosity was included by applying self-consistent formulas for $\operatorname{LD}(\Theta=0.64)$ and $\operatorname{HD}(\Theta=0.74)$ zones, as mentioned by Constantinides and Ulm (2007) and explained in chapter 1, section 1.10. Table 23 shows the elastic modulus of Tobermorite14A and Jennite with different chain lengths (Tळ, $\mathrm{T} 2$, J $\infty$ and $\mathrm{J} 2$ ). Indentation modulus (M) was calculated for comparison with experimental results performed on the cement paste surface. The indentation modulus was calculated using equation 2.2, as explained in chapter 2, section 2.5.7. The relationship between packing factor and porosity is explained in chapter 4 , section 3.9 , and is defined by the following equation:

$P=1-\theta$

In which $\mathrm{P}$ is the porosity and $\theta$ is the packing factor.

For instance, low density (LD) and high density (HD) C-S-Hs have packing factors of $\theta=0.64$ and $\Theta=0.74$, resulting in porosity of $\mathrm{P}=0.36$ and $\mathrm{P}=0.26$, respectively. Low and high density parameters were added to define elastic modulus range compared to the experimental results. 


\subsubsection{Elastic properties of Tobermorite 14A and Jennite at LD zone}

Table 20 presents the modulus of elasticity, indentation modulus and other elastic properties calculated after considering a porosity of $36 \%$.

Table 20: Elastic properties of Tobermorite and Jennite with different chain length at LD zone (P= $0.36)$

\begin{tabular}{|c|c|c|c|c|}
\hline Mineral & $\mathbf{T} \infty$ & $\mathbf{T} 2$ & $\mathbf{J} \infty$ & $\mathbf{J} 2$ \\
\hline $\mathbf{K}_{\mathbf{o}}(\mathbf{G P a})$ & 40.9 & 42.9 & 73.3 & 71.4 \\
\hline $\mathbf{G}_{0}(\mathbf{G P a})$ & 16.7 & 12.8 & 23.9 & 16.8 \\
\hline Knew(GPa) & 30.32 & 35.22 & 53.41 & 58.18 \\
\hline Gnew(GPa) & 4.91 & 3.84 & 7.14 & 5.1 \\
\hline Enew (GPa) & 14 & 11.11 & 20.51 & 14.86 \\
\hline$v$ & 0.423 & 0.447 & 0.436 & 0.457 \\
\hline M (GPa) & 17.02 & 13.89 & 25.32 & 18.80 \\
\hline Stifness & $\mathbf{L S}$ & $\mathbf{L S}$ & $\mathbf{L S}$ & $\mathbf{L S}$ \\
\hline
\end{tabular}

Table 20 shows the high impact of the new porosity parameter on the calculation of different elastic properties. By accounting for porosity according to C-S-H LD (porosity $=0.36$ ), the Young modulus of $\mathrm{T} \infty, \mathrm{T} 2, \mathrm{~J} \infty$ and $\mathrm{J} 2$ decreased by around $69 \%$ compared to the simulation that did not account for it. These high decrement percentages reconfirm the importance of density on elastic modulus results. When considering the targeted Young modulus of C-S-H, it can be noted that regardless of J $\infty$ results, Young modulus results of Tobermorite 14A and Jennite with different chain lengths (Tळ, T2 and J2) were slightly lower than the reported mean Young modulus of C-S-H, which ranged between 20 and $30 \mathrm{GPa}$. However, when considering indentation modulus (M) values, which wereequal to $17.02 \mathrm{GPa}$, 13.89 GPa, 25.32 GPa and 18.8 GPa for $\mathrm{T} \infty, \mathrm{T} 2, \mathrm{~J} \infty$ and $\mathrm{J} 2$, respectively, the mapping of those values 
into the experimental C-S-H phases completed by Constantinides and Ulm (2007) (Figure 59), showed that all the analog C-S-H minrelas ranged within the low stiffness $\mathrm{C}-\mathrm{S}-\mathrm{H}$ zone $(13<\mathrm{M}<26$ GPa). This indicates that all Young modulus values found after accounting for Tobermorite 14A and Jennite porosity were in the range that characterized the C-S-H LD zone, which confirms that the objective from accounting the porosity has been reached.

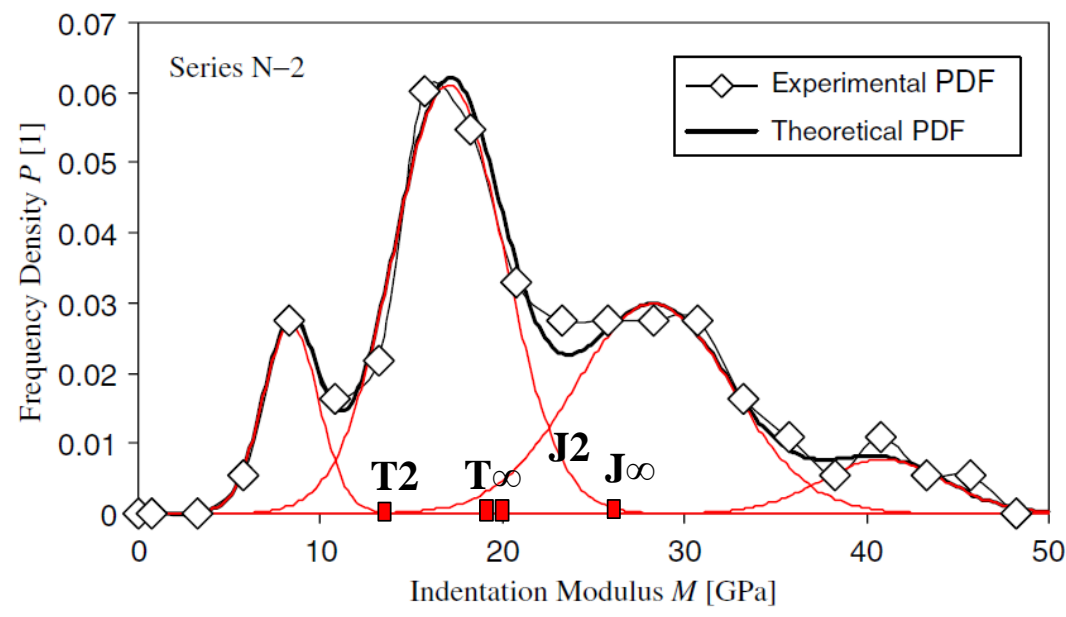

Figure 59: Location of indentation modulus of different Tobermorite 14A and Jennite samples in experimental C-S-H phases, $(\mathrm{P}=0.36 \%)$.

\subsubsection{Elastic properties of Tobermorite 14A and Jennite at HD zone}

The elastic properties of Tobermorite $14 \mathrm{~A}$ and Jennite, calculated with a porosity of $26 \%$, are presented in Table 21. 
Table 21: Elastic properties of Tobermorite and Jennite with different chain length at HD zone $(\mathrm{P}=$ $0.26)$

\begin{tabular}{|c|c|c|c|c|}
\hline Mineral & $\mathbf{T} \infty$ & T 2 & $\mathbf{J} \infty$ & $\mathbf{J} 2$ \\
\hline $\mathbf{K}_{\mathbf{o}}(\mathbf{G P a})$ & 40.9 & 42.9 & 73.3 & 71.4 \\
\hline $\mathbf{G}_{0}(\mathbf{G P a})$ & 16.7 & 12.8 & 23.9 & 16.8 \\
\hline Knew(GPa) & 33.73 & 38.23 & 59.67 & 63.26 \\
\hline Gnew(GPa) & 8.29 & 6.44 & 11.98 & 8.52 \\
\hline Enew (GPa) & 23.0 & 18.29 & 33.68 & 24.46 \\
\hline$v$ & 0.386 & 0.42 & 0.405 & 0.435 \\
\hline $\mathbf{M}(\mathbf{G P a})$ & 27.02 & 22.22 & 40.33 & 30.19 \\
\hline Stiffness & HS & $\mathbf{L S}$ & $>\mathrm{HS}$ & HS \\
\hline
\end{tabular}

LS: $\quad 13<\mathrm{M}<26 \mathrm{GPa}$ (Low stiffness)

HS: $\quad 26<\mathrm{M}<36 \mathrm{GPa}$ (High stiffness)

Table 21 shows that in considering the porosity characteristics of $\mathrm{C}-\mathrm{S}-\mathrm{H}$ HD ( $\mathrm{P}=26 \%)$, the modulus of elasticity of Tobermorite 14A and Jennite decreased considerably compared to the values from the dynamic simulation mentioned in Table 20 (which did not account for porosity). The decrement percentage was around $48 \%$ for all Young modulus results, which is lower than that shown when CS-H LD was considered (69\%). However, it remains a high decrement percentage, especially considering that $26 \%$ porosity is the lowest experimental percentage shown for C-S-H (Jennings, 2000).

Table 21 also shows that at low density $(\mathrm{p}=0.26)$, regardless of the slightly higher value of $\mathrm{J} \infty$, Young modulus results of Tobermorite 14A (Tळ, T2) and Jennite (J2) fell in the range of mean C-S-H values. In addition, when inserting indentation modulus (M) values (equal to $27.02 \mathrm{GPa}, 22.22 \mathrm{GPa}, 40.33$ GPa and 30.19 GPa for T $\infty, \mathrm{T} 2, \mathrm{~J} \infty$ and J2, respectively, in the experimental C-S-H phases completed by Constantinides and Ulm, 2007, Figure 60), T $\infty$ and J2 fell into the high stiffness range, HD C-S- 
$\mathrm{H}$ and $\mathrm{T} 2$ were in the low stiffness range, whereas, $\mathrm{J} \infty$ exceeded the range of HS C-S-H. When applying the low porosity of $\mathrm{C}-\mathrm{S}-\mathrm{H}(\mathrm{P}=0.26)$ to the elastic properties of analog $\mathrm{C}-\mathrm{S}-\mathrm{H}$ minerals, the Young modulus of infinite and finite silicate chain Tobermorite 14A (To and T2) ranged within C$\mathrm{S}-\mathrm{H}$ values at its low and high stiffness state, respectively. Young modulus of Jennite with finite silicate chain (J2) ranged within the C-S-H values, while Jennite with infinite silicate chain did not match with either low stiffness neither high stiffness C-S-H values.

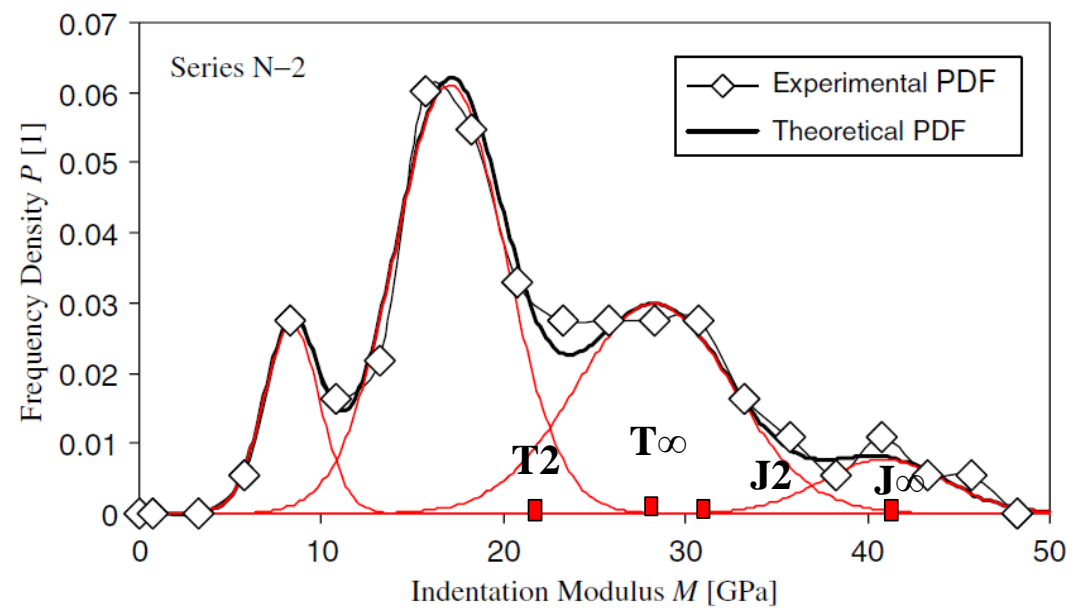

Figure 60: Location of indentation modulus of different Tobermorite 14A and Jennite samples in the experimental C-S-H phases, $(\mathrm{P}=0.26 \%)$.

\subsection{Relationship between Young modulus and packing factors}

The relationship between Young modulus values and packing factors of LD C-S-H $(\Theta=0.64)$ and HD C-S-H $(\Theta=0.74)$ is presented in Figure 61.

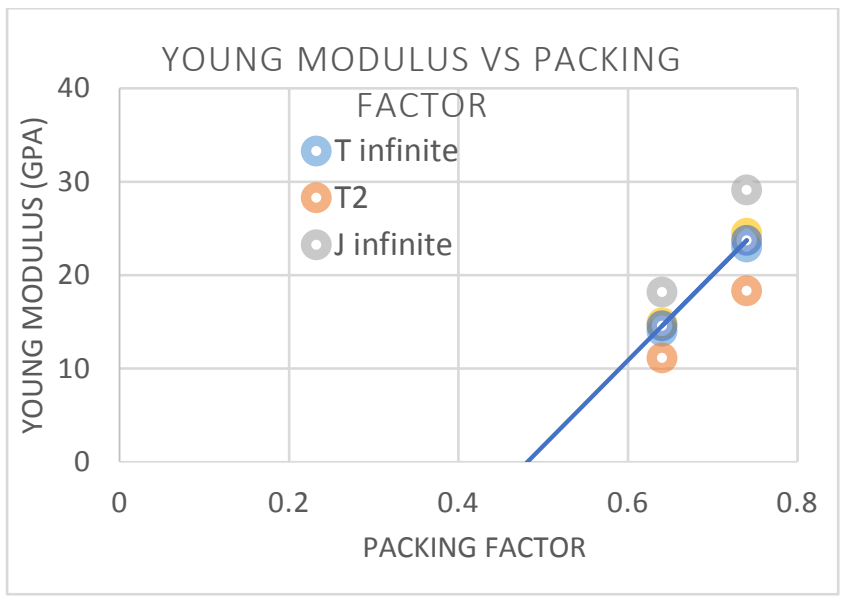

Figure 61: Young modulus vs. packing factor 
Based on the correlation presented in Figure 61, it can be observed that as packing factor increased, Young modulus also increased for all analog $\mathrm{C}-\mathrm{S}-\mathrm{H}$ minerals, becoming equal to zero when the packing factor reached a value of 0.5 . This result agrees with conclusions made by Constantinides, et al. (2007), who found that this behavior can be justified by the characterstics of the granular material, which becomes unstable when packing density is loosened.

\subsection{Discussion}

After removing the bridging silicate chain and applying the low and high porosity that characterize C-S-H (measured experimentally), the Young modulus of Tobermorite 14A with infinite and finite silicate chain (To and T2) fell within the range of HS C-S-H and LS C-S-H (high and low stiffness), whereas for Jennite, only the J2-like structure was within the range of HS C-S-H.

Jennite $\mathbf{J} 2$ structure with finite silicate chain showed an indentation modulus of $18.80 \mathrm{GPa}$ and 30.19 GPa with high $(\mathrm{P}=0.36)$ and low $(\mathrm{P}=0.26)$ porosity. Those values indicate that the Young modulus of Jennite $\mathrm{J} 2$ falls in the range of LS C-S-H and HS C-S-H when the high and low porosity are considered, respectively. Because the Young modulus of Jennite $\mathrm{J} 2$ covered both stiffness ranges and its $\mathrm{C} / \mathrm{S}$ ratio (2.25) was within the limit of $\mathrm{C} / \mathrm{S}$ ratios of $\mathrm{C}-\mathrm{S}-\mathrm{H}$, Jennite $\mathrm{J} 2$ structure can be considered the closer mineral to C-S-H (LD C-S-H and HD C-S-H) when porosity if accounted for. However, although $\mathrm{J} 2$ matched the high stiffness zone of C-S-H, it could not reach the maximum stiffness of $\mathrm{C}-\mathrm{S}-\mathrm{H}$ defined by its maximum indentation modulus $(\mathrm{M}=36 \mathrm{GPa})$. Longer silicate chains may be needed to attain extra stiffness such as $n=2$ (named pentamer, as shown in Figure 13, chapter 2, section 2.5.6). The pentamer chain is composed of five silicate tetrahedrals rather than the two used in this study. This explanation agrees with the results of the experimental investigation performed on C-SH by Richardson (1999). Indeed, by using the trimethylsilylation (TMS) technique and nuclear magnetic resonance (NMR) spectroscopy, Richardson showed that the dimeric silicate chain $(n=1)$ is the predominate species in $\mathrm{C}-\mathrm{S}-\mathrm{H}$, formed during the first 24 hours after mixing. However, any C-S- 
$\mathrm{H}$ formed after that period, and even after six or more months of hydration, consists of mainly linear polymeric species such as pentamer.

Tobermorite T2 with finite silicate chain showed an indentation modulus (M) of $13.89 \mathrm{GPa}$ and 22.22 $\mathrm{GPa}$ when applying the low and high porosities that characterize C-S-H. These values fell in the range of 13 and $26 \mathrm{GPa}$, which represents the low stiffness area of $\mathrm{C}-\mathrm{S}-\mathrm{H}$. This result indicates that Tobermorite-like structures (T2) cannot reach the high stiffness zone of C-S-H (HS C-S-H). According to Jennings (2000), C-S-H with low density (low stiffness) forms faster during the early hydration age, while high density C-S-H (high stiffness) takes longer to form (potentially over days). This suggests that the T2 resulting from this study only represents the LS C-S-H formed during the early age of hydration.

When applying the low porosity to the Jennite Jo structure, the Young modulus value was $20.51 \mathrm{GPa}$, which falls within the low stiffness range of C-S-H. However, at high porosity, the Young modulus of Jennite J $\infty$ was estimated to be $33.68 \mathrm{GPa}$, which exceeds the high stiffness range. With a minimum porosity $\mathrm{p}=0.26$, Jennite $\mathrm{J} \infty$ cannot be in the HD C-S-H range. The bridging silicate chain must be removed to reduce the Young modulus.

Tobermorite T $\infty$ with infinite silicate chain length showed a Young modulus of $14 \mathrm{GPa}$ and $23 \mathrm{GPa}$. When applying low and high porosity on its structure, the Young modulus of Tobermorite To fell within the low and high stiffness ranges of C-S-H. This indicates that Tobermorite T $\infty$ represents the structure of C-S-H in its low and high densities. However, by considering the low $\mathrm{C} / \mathrm{S}$ ratio of this mineral (equal to 0.83 ), the structure of Tobermorite 14A with infinite silicate chain cannot represent $\mathrm{C}-\mathrm{S}-\mathrm{H}$ structure, because the $\mathrm{C} / \mathrm{S}$ ratio of $\mathrm{C}-\mathrm{S}-\mathrm{H}$, evaluated experimentally, ranges between 1.2 and 2.3.

\subsection{Summary}

Tobermorite 14A and Jennite were found to be the minerals closest to C-S-H in structure. However, their Young modulus values were higher than that of $\mathrm{C}-\mathrm{S}-\mathrm{H}$. The objective of this chapter was to 
reduce the Young modulus of Tobermorite 14A and Jennite using dynamic simulation by COMPASS force field, to be closer to the experimentally measured Young modulus of $\mathrm{C}-\mathrm{S}-\mathrm{H}$. The reduction of elastic modulus was attempted using two approaches. In the first method, a molecular dynamic simulation with COMPASS force field was run on Tobermorite 14A and Jennite structures with finite silicate chain, in which the bridging silicate chain had been removed. In the second method, the porosity of Tobermorite 14A and Jennite were accounted for.

In the first approach, after omitting the bridging silicate, Tobermorite $\mathrm{T} 2$ and Jennite $\mathrm{J} 2$ were the new defect structures created from Tobermorite 14A and Jennite. Tobermorite T2 and Jennite J2 are characterized by a dimer silicate chain $(n=1)$. The removal of the bridging silicate chain caused reductions of $20 \%$ and $28 \%$ in the Young modulus of Tobermorite 14A and Jennite, respectively. The cause of the reduction in Young modulus after omitting the bridging silicate can be attributed to a number of reasons, but reduced density due to mass loss as a result of removing the bridging silicate chain has been suggested as the principal cause. Although removing the bridging silicate chain caused remarkable reductions of Young modulus in Tobermorite 14A and Jennite, results of the molecular dynamic simulation of Tobermorite T2 and Jennite J2 by COMPASS force field (estimated to 34.93 GPa and 46.72GP, respectively), were higher than those in the mean range of C-S-H (LD and HD), measured experimentally to be between 20 and $30 \mathrm{GPa}$, respectively.

Because of the higher estimated Young modulus of Tobermorite 14A and Jennite, even after removing the bridging silicate, this study focused on the second proposed method, which was to account for porosity in all Tobermorite 14A and Jennite structures with infinite and finite silicate chains $(\mathrm{T} \infty, \mathrm{T} 2, \mathrm{~J} \infty$ and $\mathrm{J} 2)$. After applying the two types of porosity that characterize $\mathrm{C}-\mathrm{S}-\mathrm{H}$ in its low (LD) $(\mathrm{P}=0.36)$ and high density $(\mathrm{HD})(\mathrm{P}=0.26)$ states, the Young modulus of Tobermorite T $\infty$, Tobermorite T2 and Jennite J2 fell within the range of LS C-S-H (low stiffness) and HS C-S-H (high stiffness), which satisfy the goals of this study, although the Young modulus of Jennite J $\infty$ still exceeded the range of the high stiffness zone of C-S-H. 
Because the C/S ratio of Jennite $\mathrm{J} 2$ was in the range of $\mathrm{C}-\mathrm{S}-\mathrm{H}$ and since its Young modulus after accounting for porosity covered the low and high stiffness areas of $\mathrm{C}-\mathrm{S}-\mathrm{H}$, Jennite $\mathrm{J} 2$ is considered to be the closer structure to C-S-H with its LD and HD states.

The structure of Tobermorite T2 (with porosity accounted for) is also considered to be close to C-SH. However, the Young modulus of Tobermorite T2 cannot reach the HD C-S-H range, which suggests that its structure can represent only the LD C-S-H.

Although Tobermorite T $\infty$ showed a Young modulus within the range of LD and HD C-S-H after accounting for porosity, the $\mathrm{C} / \mathrm{S}$ ratio of this mineral is lower than that of $\mathrm{C}-\mathrm{S}-\mathrm{H}$. This suggests that the structure of Tobermorite 14A with infinite silicate chain cannot represent the structure of C-S-H (either LD or HD C-S-H).

The Young modulus of Jennite $\mathrm{J} \infty$ exceeded the limit of C-S-H even after accounting for porosity, which indicates that Jennite with infinite silicate chain cannot represent $\mathrm{C}-\mathrm{S}-\mathrm{H}$ unless the bridging silicate chain is removed and the porosity is accounted for. 


\section{Chapter 8 General Conclusions}

\subsection{General conclusions}

Concrete or cement paste is viewed only at macro scale; however, the atomic scale controls its engineering properties. Molecular simulation is the tool used to investigate the interaction between atoms. This thesis is focused on the mechanical properties of clinker phases and hydration products such as Portlandite, Ettringite and C-S-H. Because the lack of models of the atomic structure of C-S$\mathrm{H}$ remains an obstacle to concrete optimization, special attention is given to this significant component of cement hydration. An understanding of the nanostructure of $\mathrm{C}-\mathrm{S}-\mathrm{H}$, the main binding phase and critical hydration product, can help us design proper cement paste formulations. However, due to its amorphous and unstable structure, the nano- and micro-structure of C-S-H has been more frequently studied using experimental techniques such X-ray diffraction (XRD), scanning probe microscopy (SPM) and Nuclear Magnetic Resonance (NMR). To counter its complicated structure, most of the current literature on C-S-H modeling has been drawn from other minerals that are structurally analog to C-S-H structure. Tobermorite family and Jennite are the nano-crystals most commonly used in the structural modeling of C-S-H. The numerical simulation of those minerals can help resolve the problem of C-S-H structure at its nano-scale level. The goal of this thesis was to use the molecular simulation by COMPASS force field in material studio software to numerically study the nanostructure of clinker minerals, Portlandite, Ettringite and, especially C-S-H to determine their elastic properties. The relationship between $\mathrm{C} / \mathrm{S}$ ratio, packing factor and silicate chain length in $\mathrm{C}$ S-H structure and its modulus of elasticity we also targeted. 
Like the numerical methods used in literature to characterize the $\mathrm{C}-\mathrm{S}-\mathrm{H}$ structure, the molecular dynamic simulation by COMPASS force field used in this study was based on the numerical simulation of Tobermorite family and Jennite. The optimization of the geometry was the first step in all the computation work, and thereafter the dynamic simulation was performed in two steps: equilibrium and production. The unit cell and lattice parameters for different clinker phases $\left(\mathrm{C}_{3} \mathrm{~S}\right.$, $\mathrm{C}_{2} \mathrm{~S}, \mathrm{C}_{3} \mathrm{~A}$ and $\mathrm{C}_{4} \mathrm{AF}$ ), hydration products (Portlandite and Ettringite), and analog $\mathrm{C}-\mathrm{S}-\mathrm{H}$ minerals (Tobermorite 9A, 11A and 14A and Jennite) were selected from literature. The final geometry optimization and dynamic simulation parameters were chosen according to adequate simulation time and accurate physical structure. The elastic properties resulting from the dynamic simulation were compared with those of the experimental results mentioned in the literature. The final elastic modulus of the studied elements was calculated by considering the porosity of the cement paste using a special equation.

Based on the numerical results, the following conclusions can be drawn:

1- There was good agreement between the molecular modeling and the experimental results of the elastic modulus of the clinker phases. However, no clear relationship was noticed between $\mathrm{C} / \mathrm{S}$ ratio and elastic modulus of anhydrate cement.

2- The elastic properties of Ettringite and Portlandite were also in close agreement with the experimental results. The elastic modulus of Portlandite was confirmed to be 2.7 times higher than that of Ettringite.

3- The calculated Young modulus of Tobermorite 9A, Tobermorite 11A, Tobermorite14A, and Jennite were higher than those of C-S-H estimated experimentally. However, the results of this study aligned with the elastic properties mentioned in literature using quantum mechanics principle.

4- Two methods have been tested to reduce the Young modulus of Tobermorite 14A and Jennite to be close to that mentioned experimentally for $\mathrm{C}-\mathrm{S}-\mathrm{H}$ : breaking the silicate chain length 
following the rule $3 n-1$ and introducing porosity to the structures of Tobermorite $14 \mathrm{~A}$ and Jennite $(\mathrm{P}=0.26$ and $\mathrm{P}=0.36)$.

5- After removing the bridging, the molecular dynamic simulation of the finite silicate chain Tobermorite_type T2 and Jennite_type J2 by COMPASS force field showed Young modulus values of $34.93 \mathrm{GPa}$ and 46.72GP, respectively. The finite silicate chain resulted in reduced elastic modulus compared to the infinite silicate chain. However, even after removing the bridging silicate tetrahedral, the Young modulus of the analog $\mathrm{C}-\mathrm{S}-\mathrm{H}$ minerals remained higher than those of the experimental results performed on $\mathrm{C}-\mathrm{S}-\mathrm{H}$, in which the mean value of Young modulus ranged between 20 and 30GPa.

6- Two types of porosity were applied to infinite and finite silicate chain of Tobermorite 14A (Tळ and T2) and Jennite ( $\mathrm{J}_{\infty}$ and $\mathrm{J} 2$ ), which are specific characteristics of $\mathrm{C}-\mathrm{S}-\mathrm{H}$ at its low density (LD) $(\mathrm{P}=0.36)$ and high density (HD) $(\mathrm{P}=0.26)$ states. After accounting for the porosity, the calculated Young modulus of Tobermorite Tळ, Tobermorite T2 and Jennite J2 fit within the range of LS C-S-H (low stiffness) and HS C-S-H (High stiffness).

7- The Young modulus of $\mathbf{J} 2$ covered both the low stiffness (LD) and high stiffness zones, and its $\mathrm{C} / \mathrm{S}$ ratio was within the range of $\mathrm{C} / \mathrm{S}$ ratio of $\mathrm{C}-\mathrm{S}-\mathrm{H}$. $\mathrm{J} 2$ was confirmed to be the analog C-S-H mineral that can most closely represent the structure of C-S-H.

8- The Young modulus of the T2 structure, with porosity accounted for, was the low stiffness range of C-S-H and can therefore only represent the structure of C-S-H at early age hydration.

9- Because the C/S ratio of Tobermorite 14A was low and its Young modulus did not reach the highest stiffness zone, this analog C-S-H mineral cannot represent $\mathrm{C}-\mathrm{S}-\mathrm{H}$ structure.

10- Jennite with infinite chain $(\mathrm{J} \infty)$ cannot represent the C-S-H structure because its Young modulus was confirmed to be beyond the range of HS C-S-H (high stiffness zone).

11- Based on the molecular dynamic simulation by COMPASS force field used in this study, one of the most important parameters affecting the Young modulus of Tobermorite 9A, 11 A, 14A and Jennite was interlayer spacing, which played an important role in the direction of 
compressibility and the stiffness of the mineral. As the interlayer increased, Young modulus decreased. The direction of the compressibility was in the parallel direction of the interlayer.

12- The packing factor, which determines the morphology of C-S-H atoms, also had a considerable effect on Young modulus.

13- Water to silicate and water to calcium ratios were also major parameters that influenced elastic modulus. Excess of water can increase the spacing between the calcium silicate layers and reduce the interaction between atoms. As W/Ca and W/Si decreased in Tobermorite 14A, 11A, 9A and Jennite, the Young modulus increased.

14- Density, which determines the compactness and packing of particles, was also a major parameter affecting the elastic modulus of analog $\mathrm{C}-\mathrm{S}-\mathrm{H}$ minerals. As density increased, the Young modulus increased.

This study confirms the efficiency of the numerical method using COMPASS force field in material studio software in the estimation of the elastic properties of most clinker phases and hydration products. Unlike the different experimental and computational methods, the numerical simulation by COMPASS force field showed good results in reduced time and with minimum staff.

\subsection{Recommendations:}

For a further understanding of the mechanical performance of different types of C-S-H, more research will be needed into:

* The study of the nano-structure of C-S-H in blended cement paste or Portland cement paste with supplementary cementing materials.

* The effect of some aggressive species such as magnesium and sulfuric acid that can considerably affect the nano-structure of C-S-H.

Considering the different forms of $\mathrm{C}-\mathrm{S}-\mathrm{H}$ with the presence of high amounts of $\mathrm{Na}$, $\mathrm{K}$ or $\mathrm{Al}$, it would be prudent to investigate another C-S-H structures like C- (Na, Al)-S-H or C- (Na-K)-S-H 
Appendixes: Input parameters to Materials Studio software and elastic properties

\section{I- Alite (C3S)}

\section{I-1- Geometry optimization parameters}
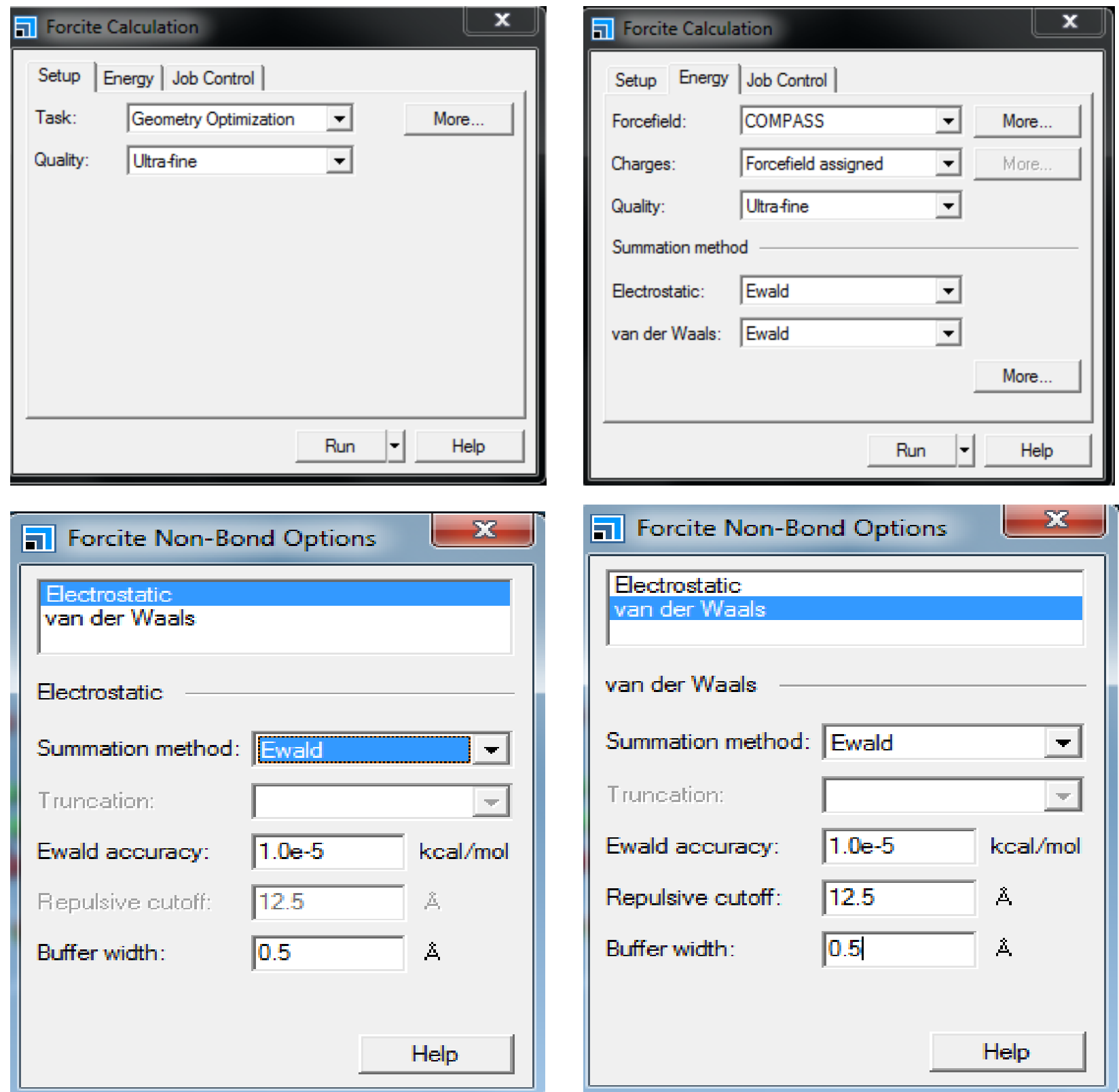


\section{I-2- Dynamic simulation parameters (C3S)}
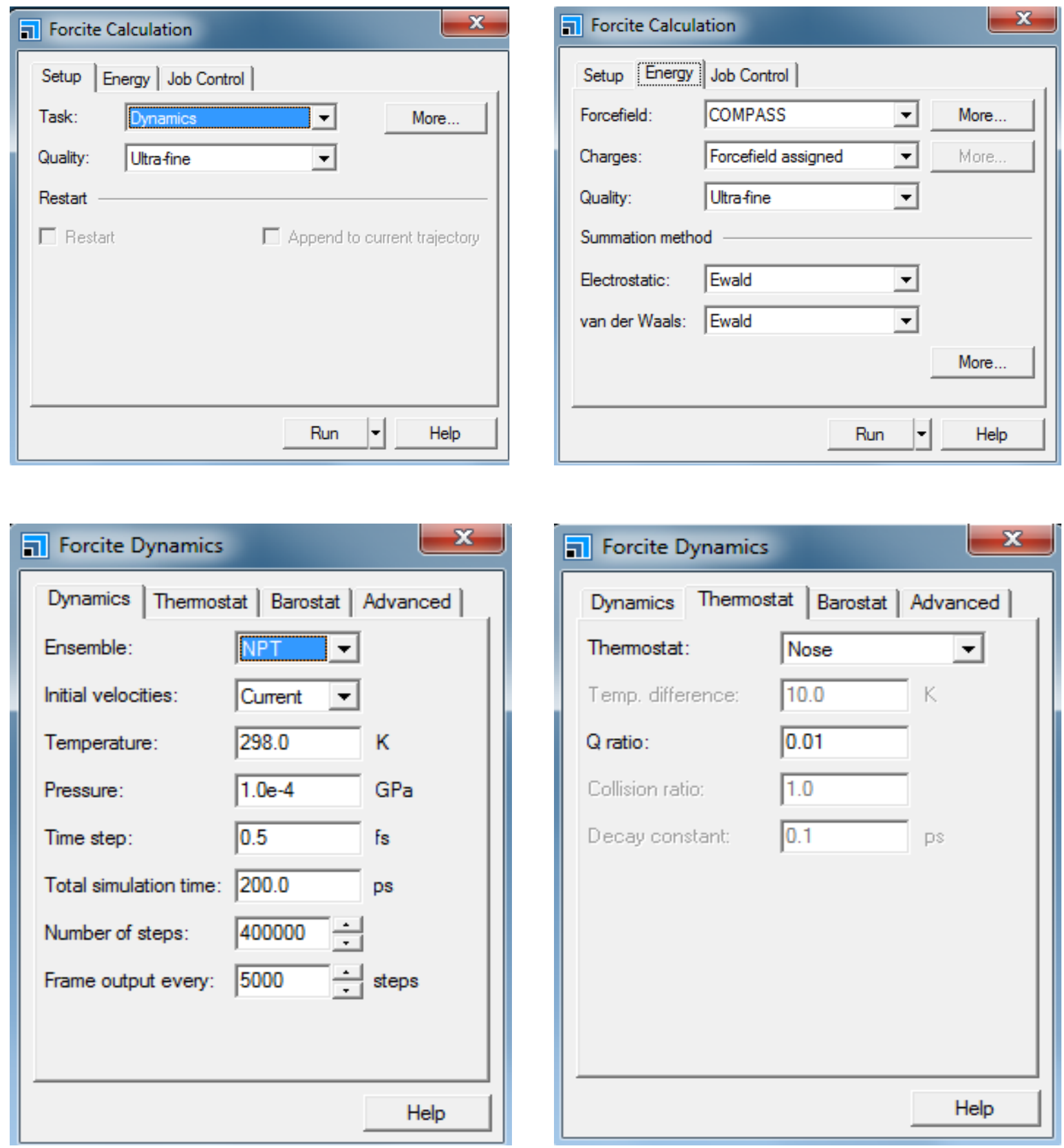

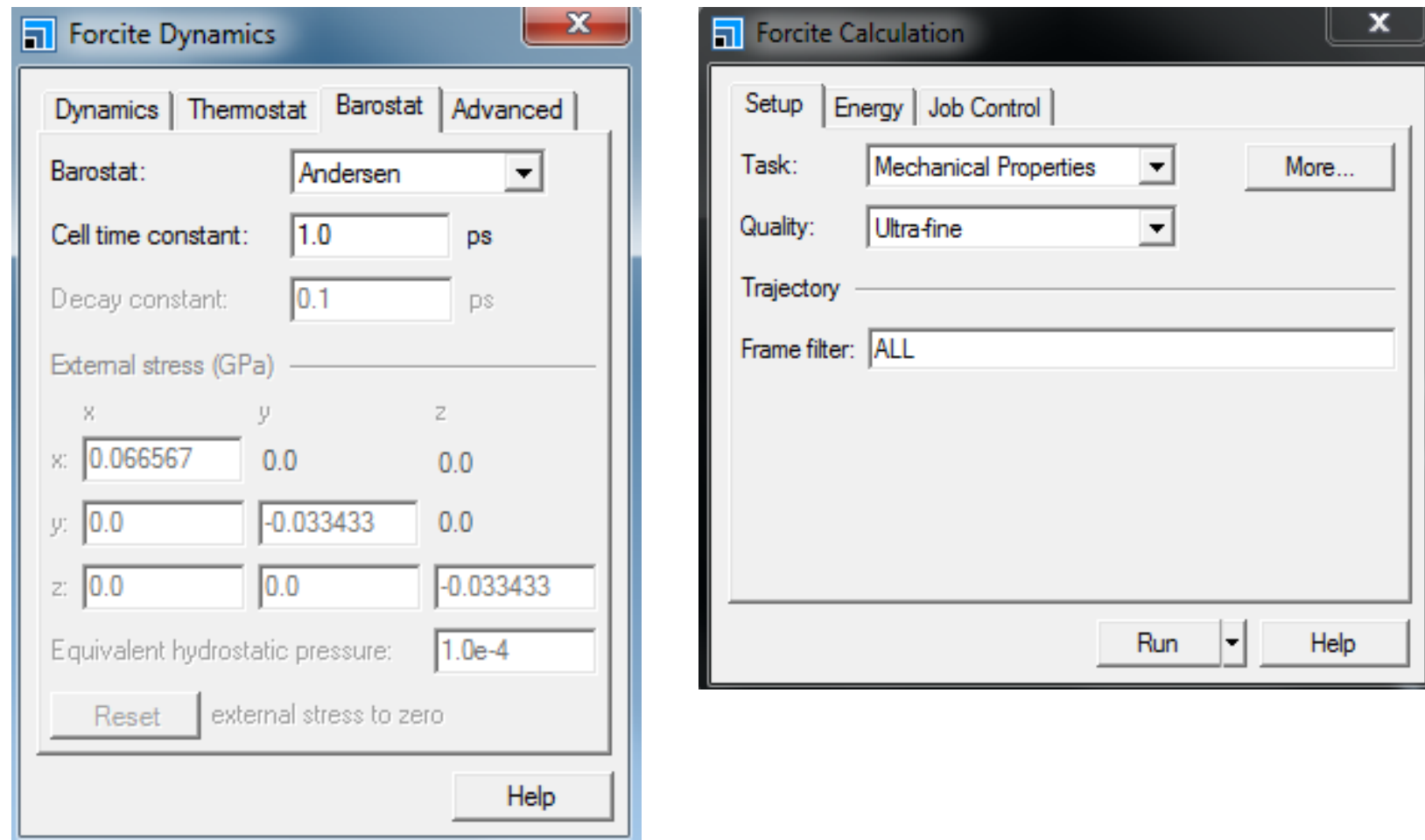

\section{I-3- Elastic properties of C3S}

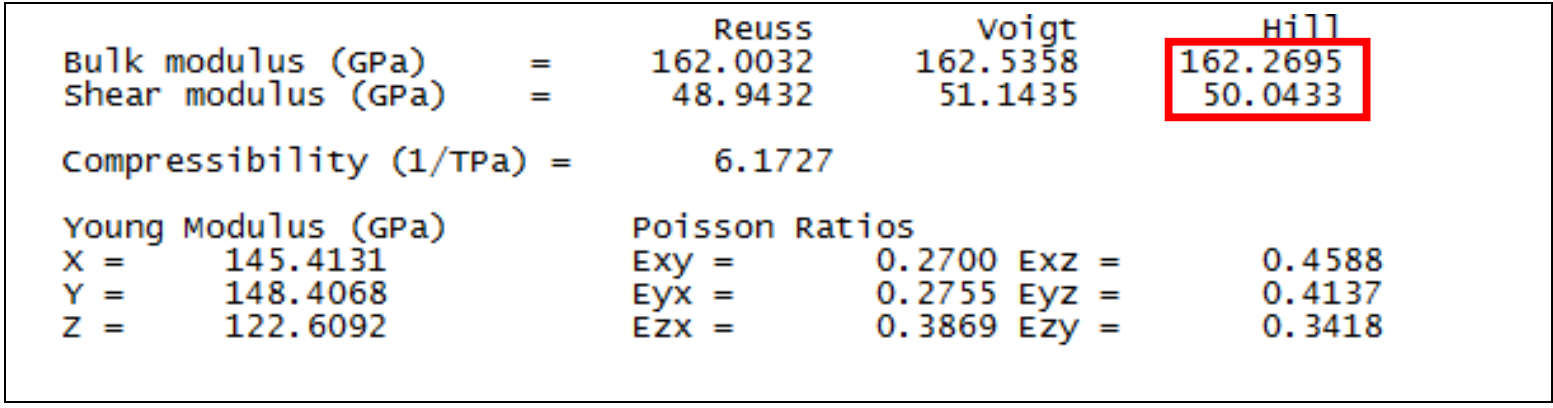




\section{II- Belite (C2S)}

\section{II-1- Geometry optimization parameters of C2S}
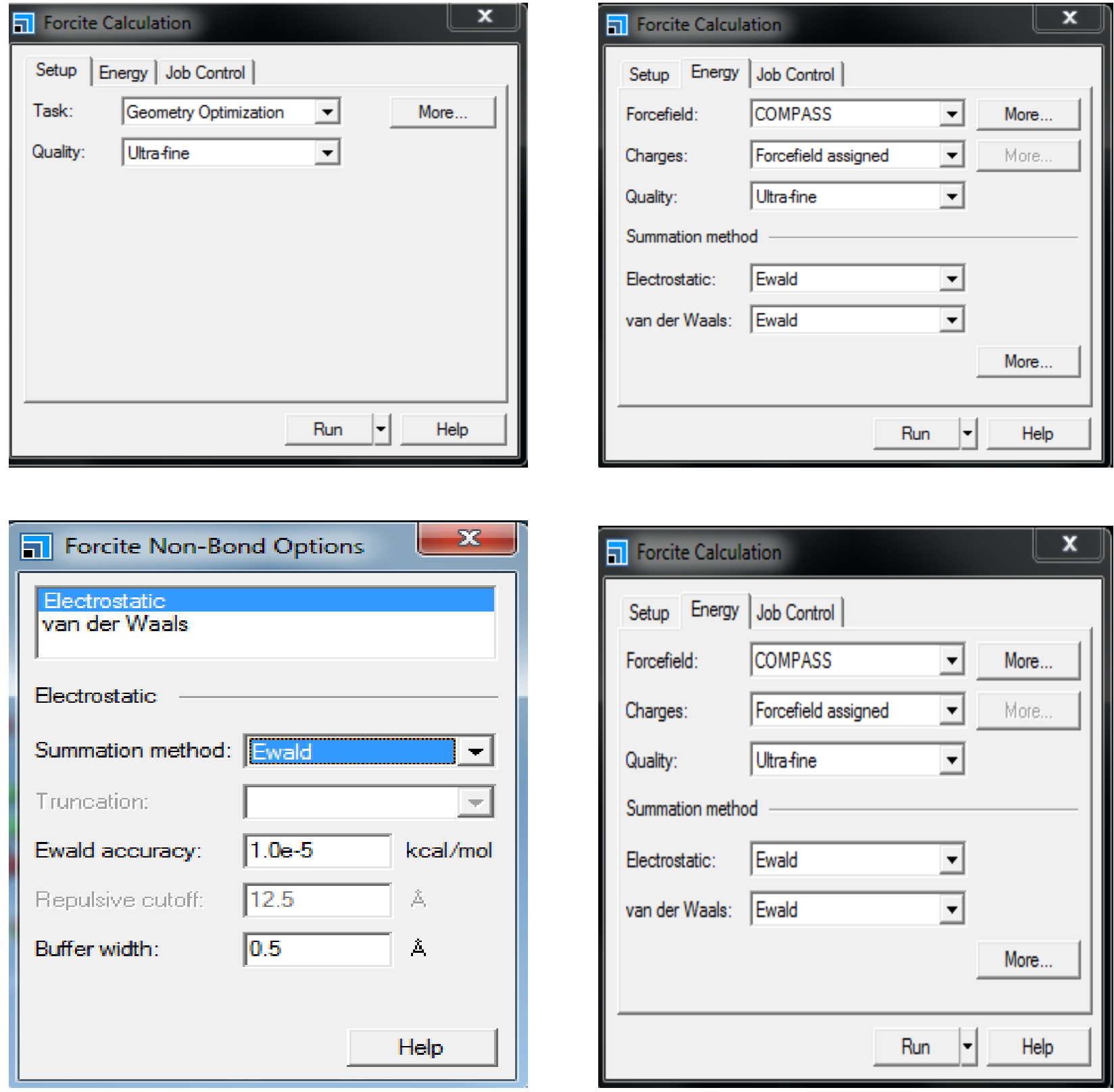


\section{II-2- Dynamic simulation (C2S)}
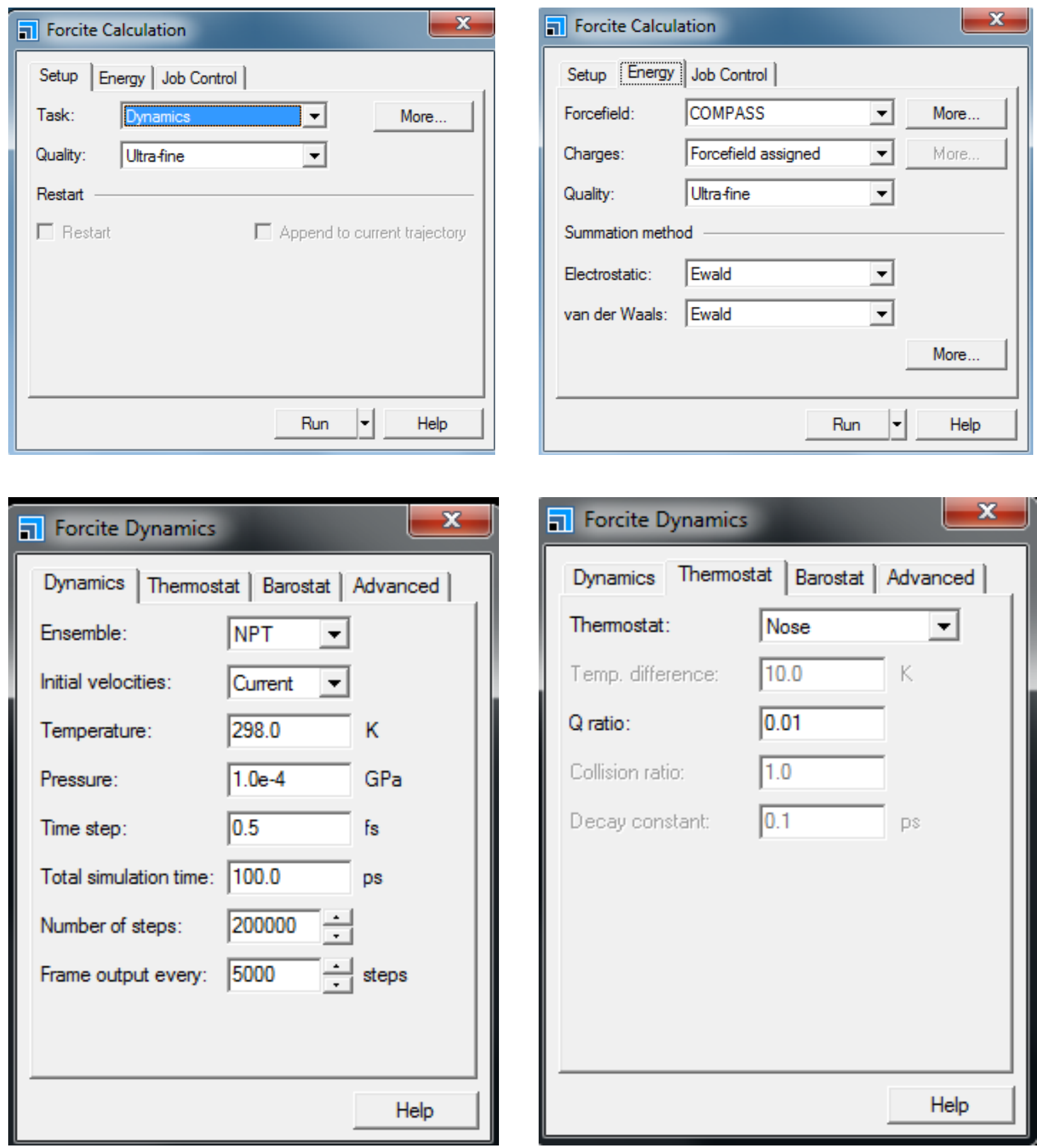

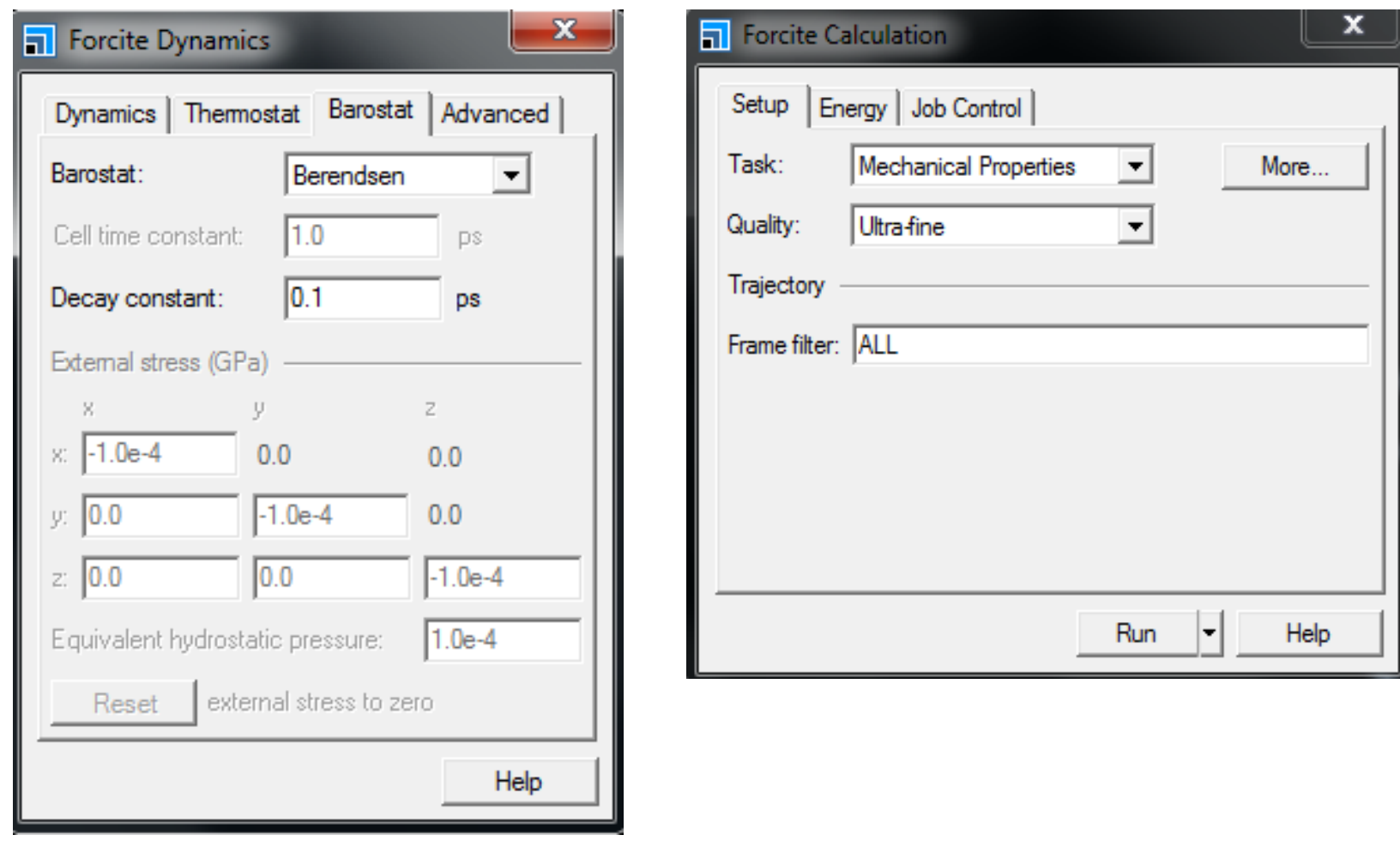

\section{II-3- Elastic properties of C2S}

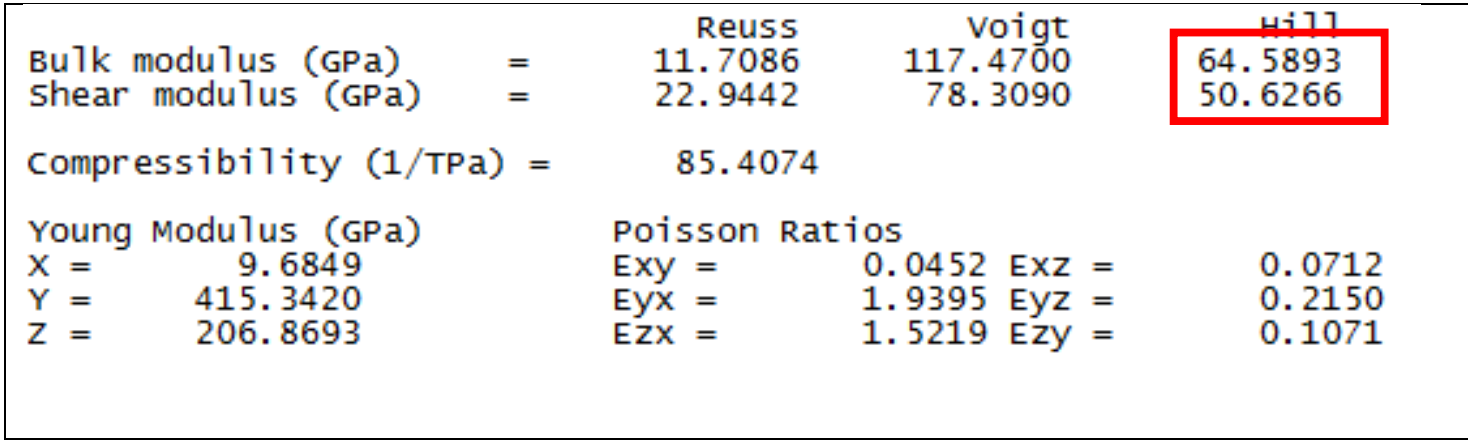




\section{III- Tricalcium Aluminate (C3A)}

\section{III-1- Geometry optimization parameters of C3A}
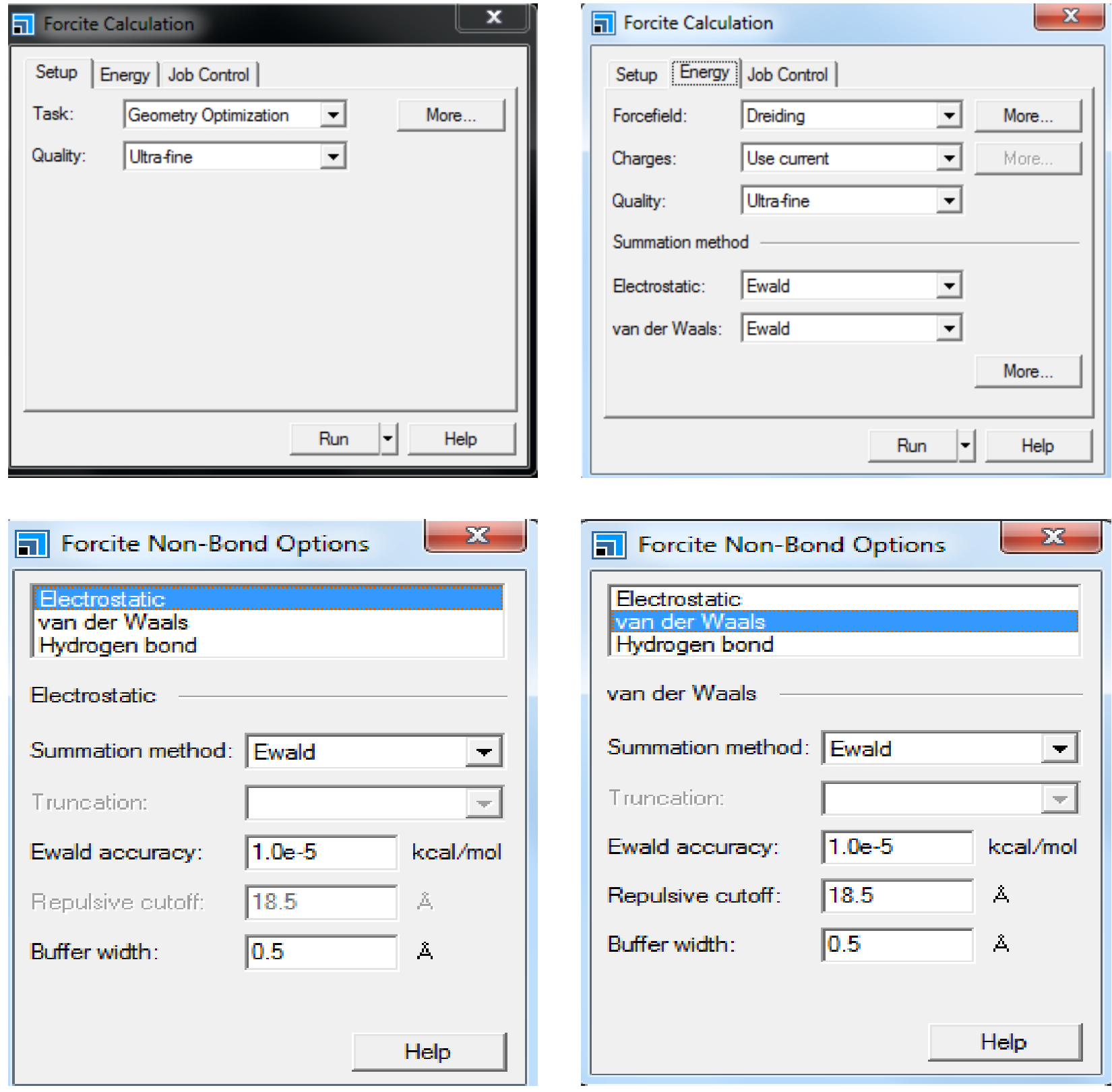
III-2- Dynamic simulation parameters of C3A
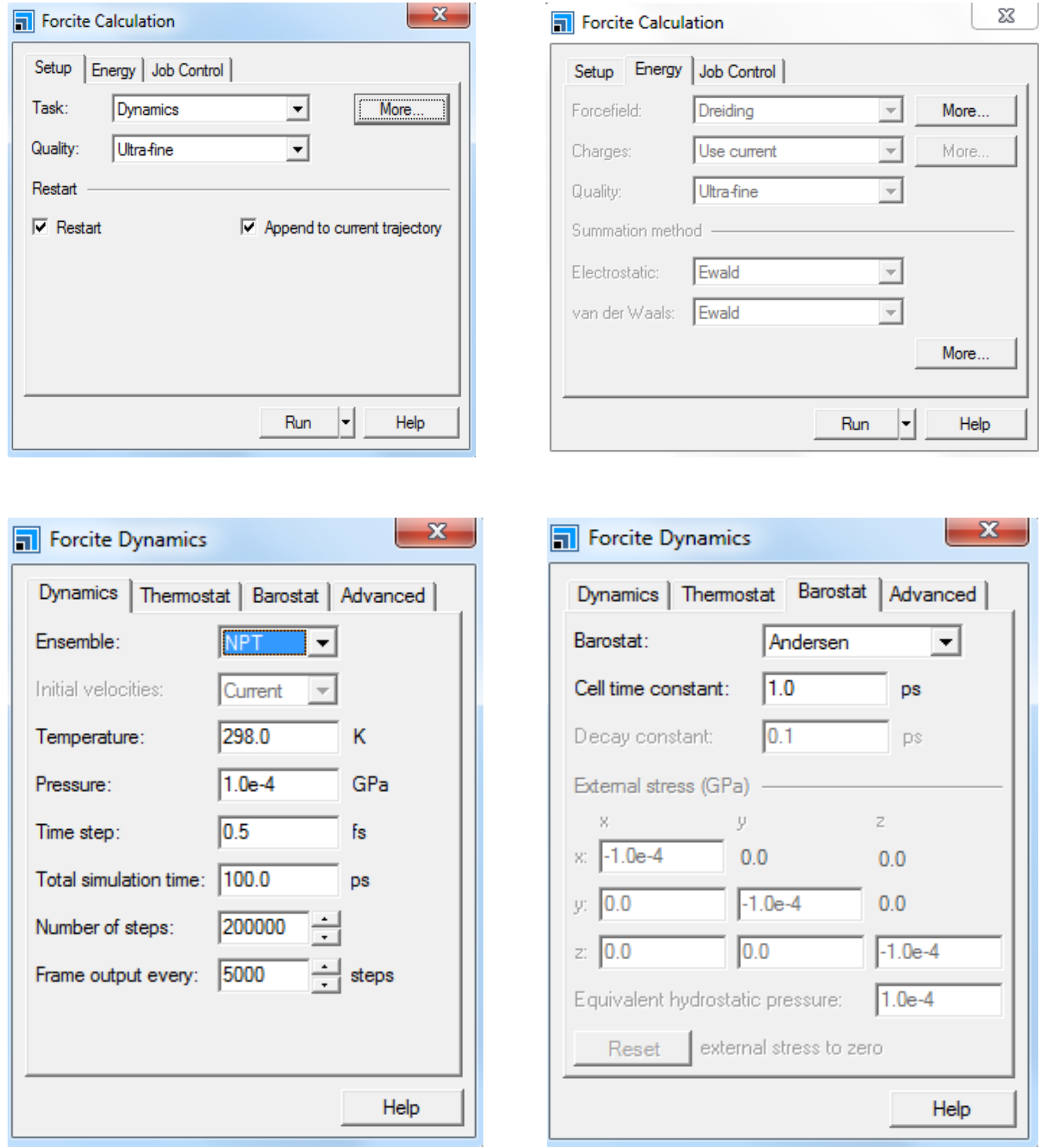

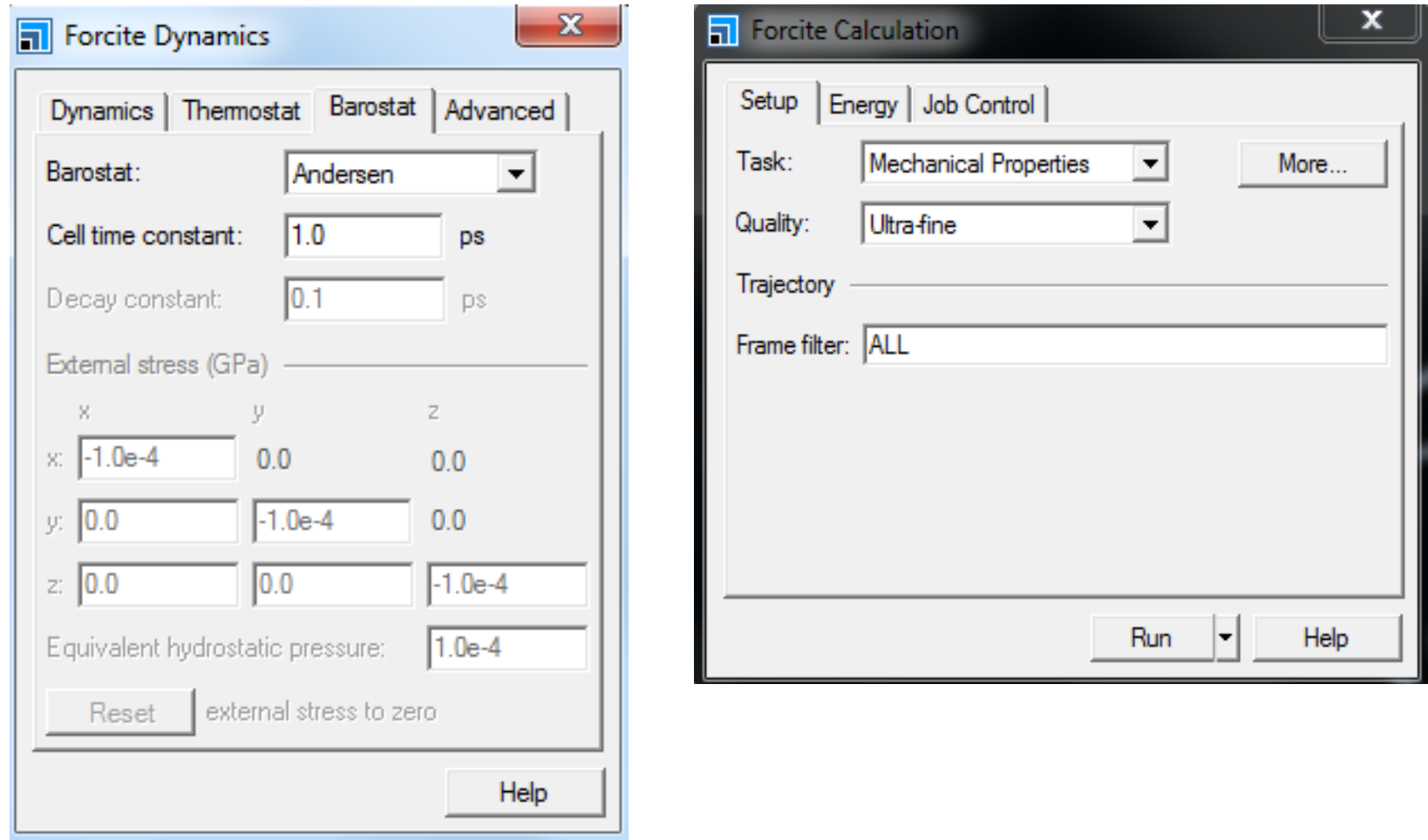

\section{III-3- Elastic properties of C3A}

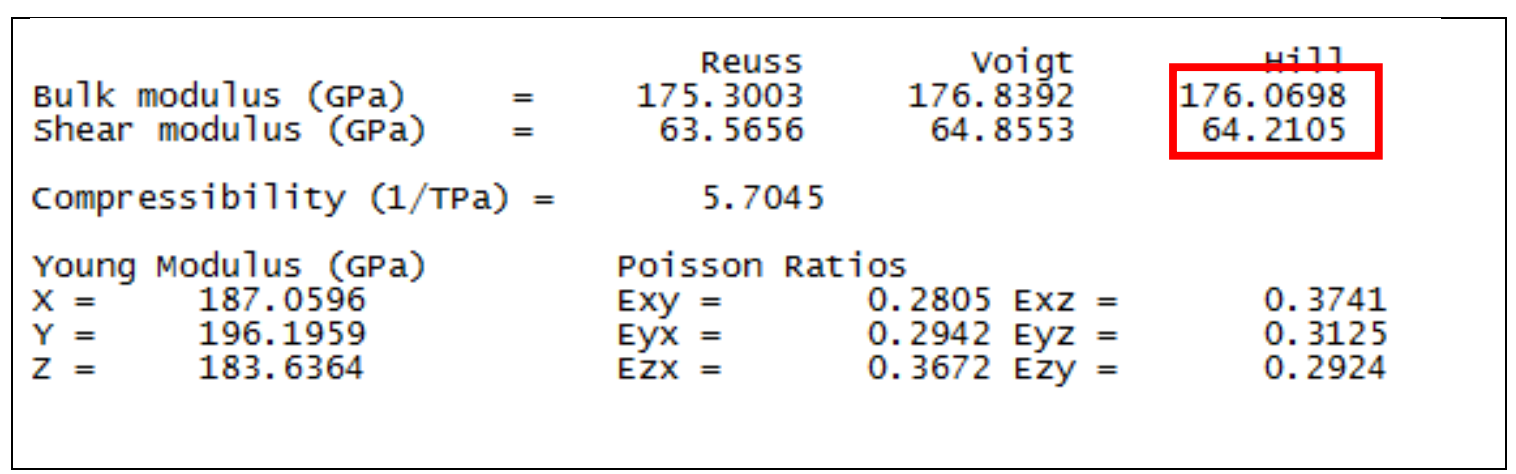




\section{IV- Aluminoferrite ( $\left.\mathrm{C}_{4} \mathrm{AF}\right)$}

\section{IV-1- Geometry optimization}
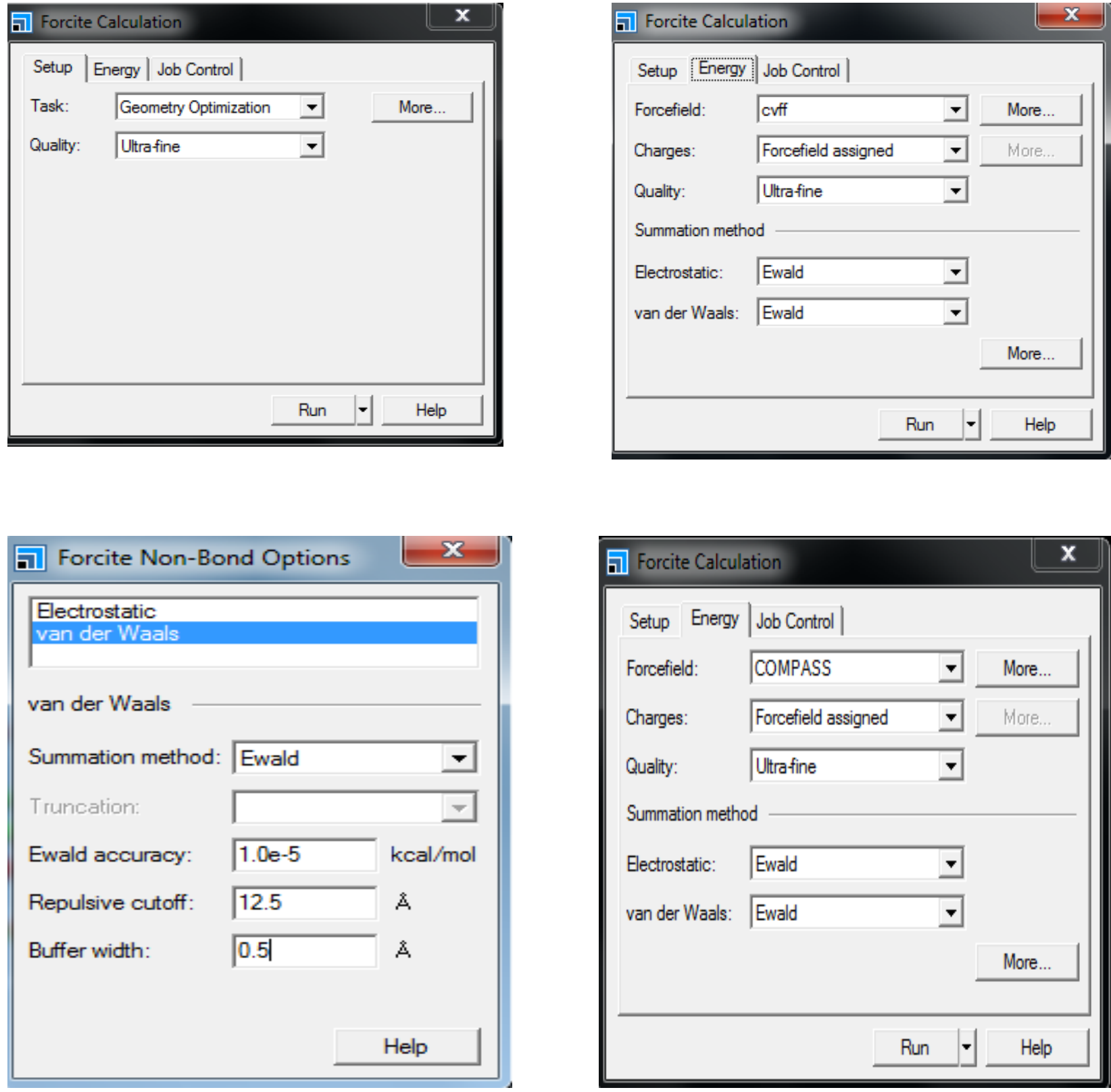


\section{IV-2- Dynamic simulation C4AF}
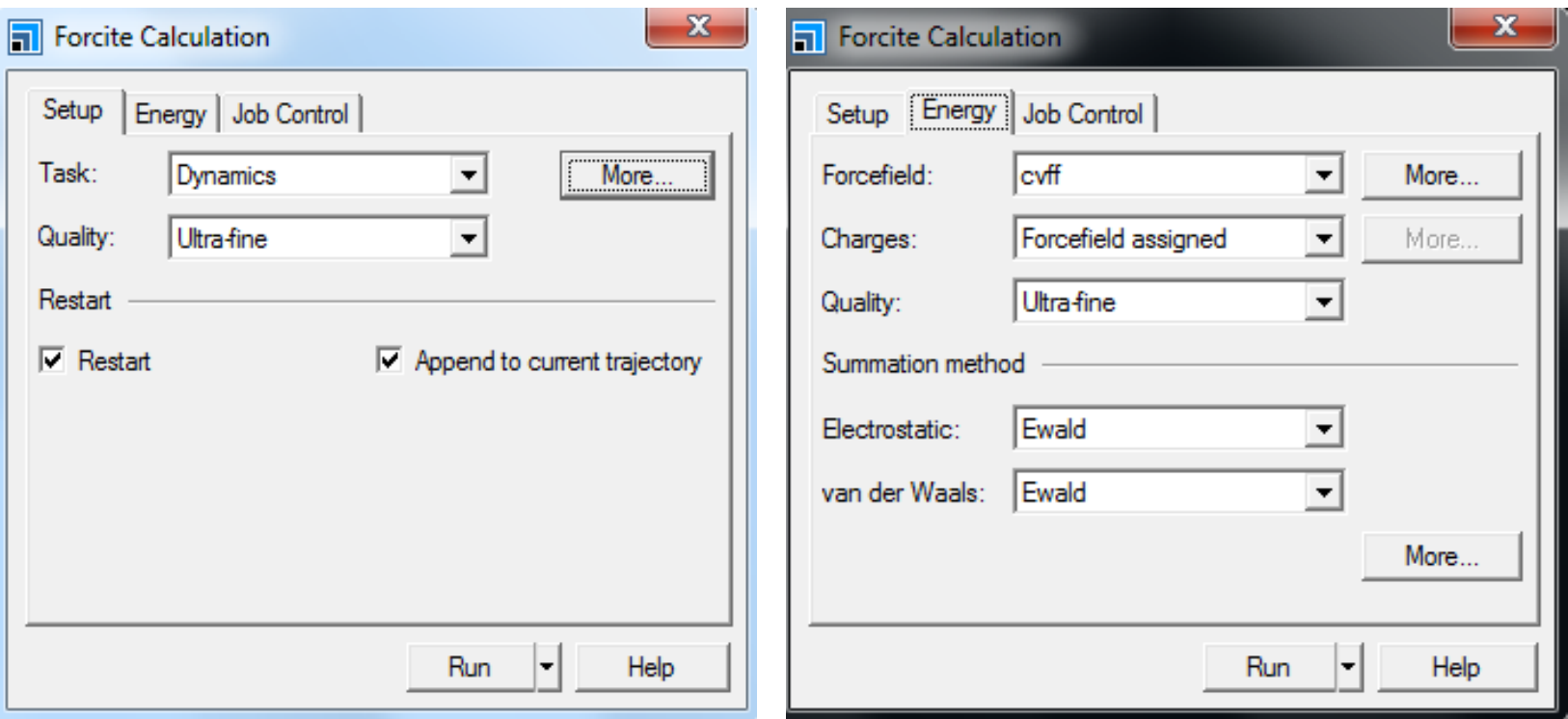

\section{目 Forcite Dynamics}

回 Forcite Dynamics

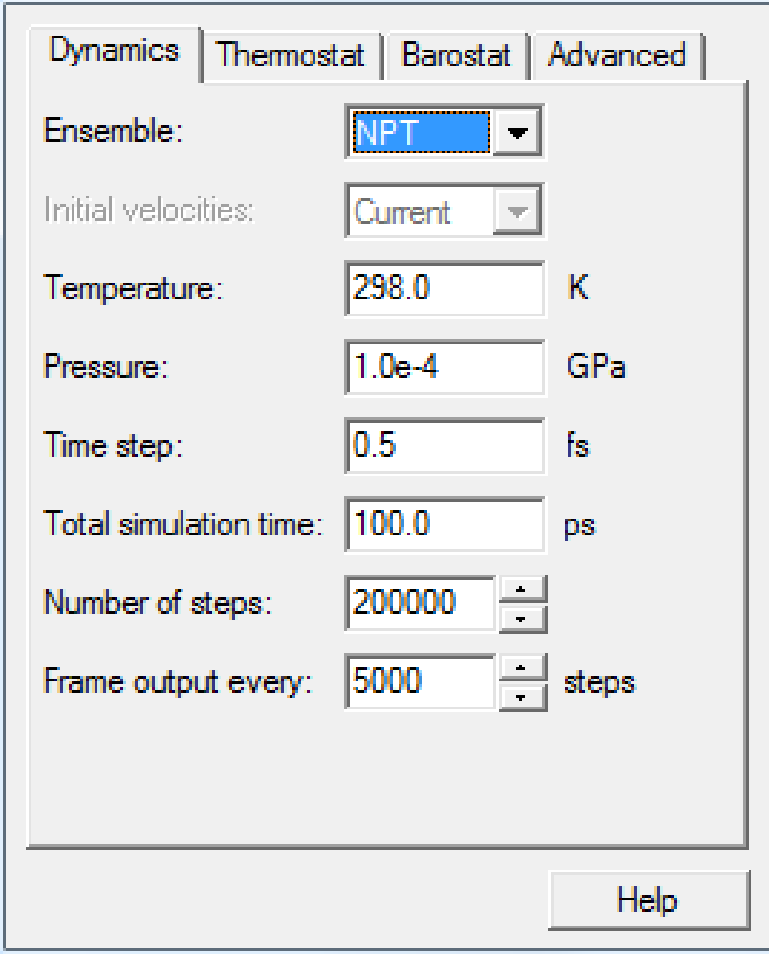

Dynamics | Thermostat Barostat |Advanced |

Barostat:

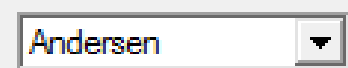

Cell time constant:

$\sqrt{1.0 \quad \text { ps }}$

Decay constant

$\sqrt{0.1}$ ps

Extemal stress (GPa)

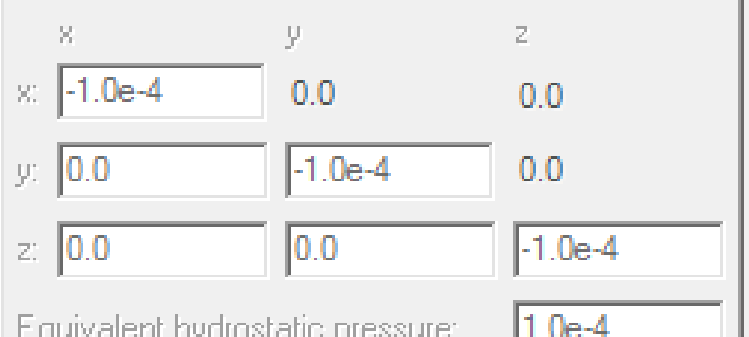

Equivalent hydrostatic pressure: $\quad 1.0 \mathrm{e}-4$

Reset external stress to zero 

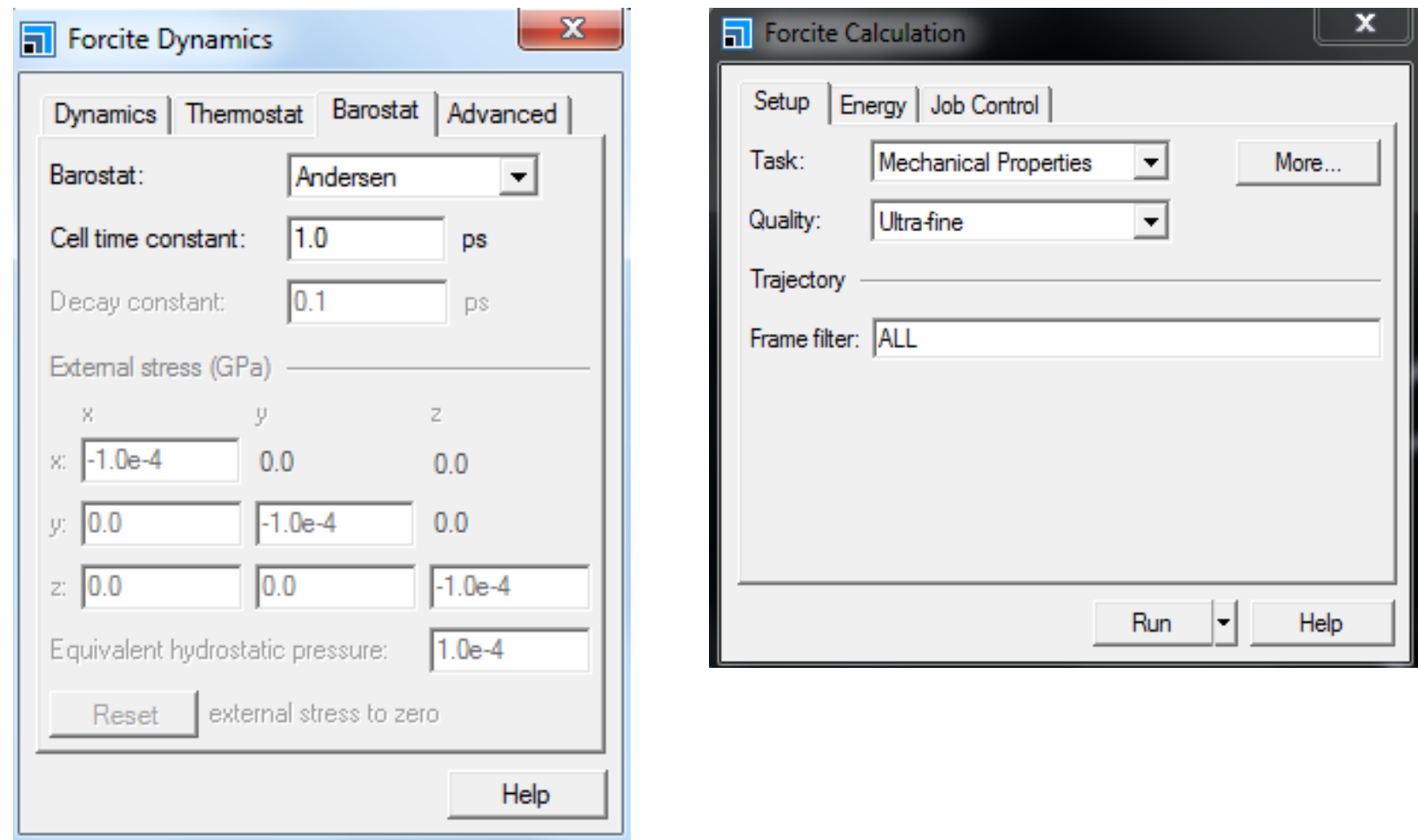

\section{IV-3- Elastic properties of C4AF}

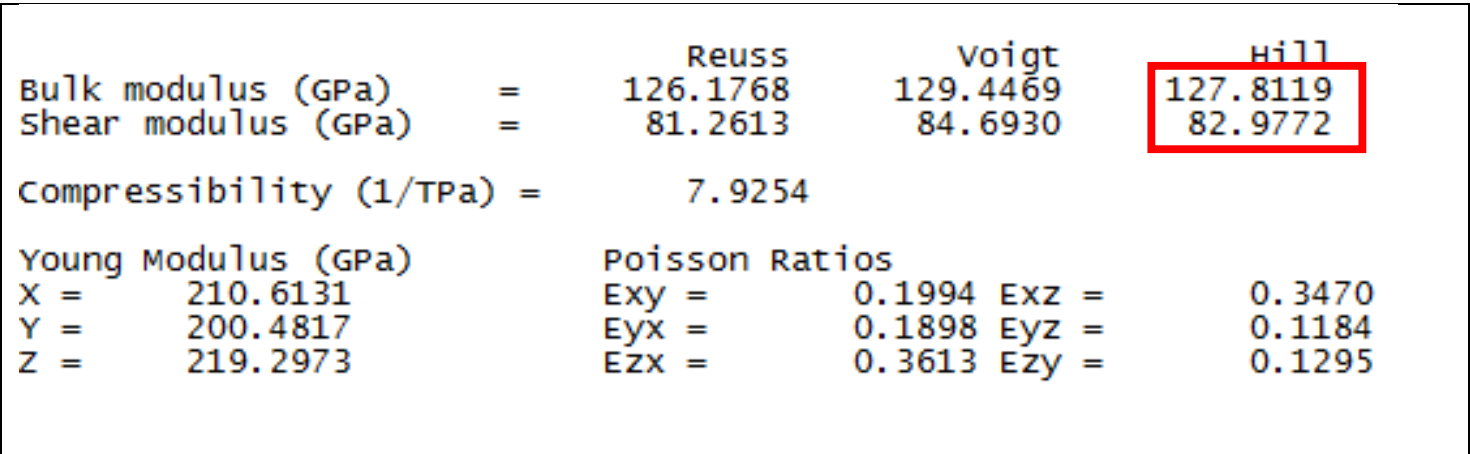




\section{V- Portlandite}

\section{V-1- Geometry optimization parameters}

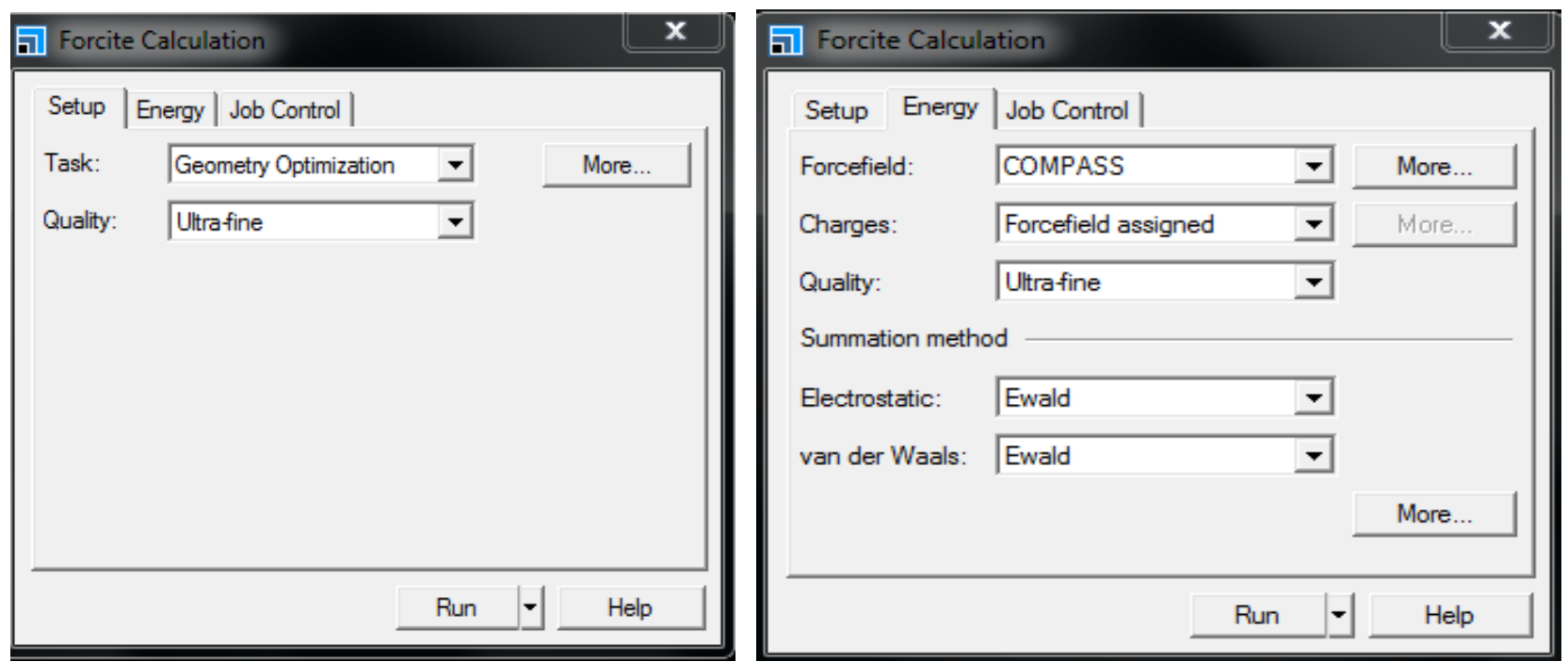

\section{V-2- Dynamic simulation (Portlandite)}

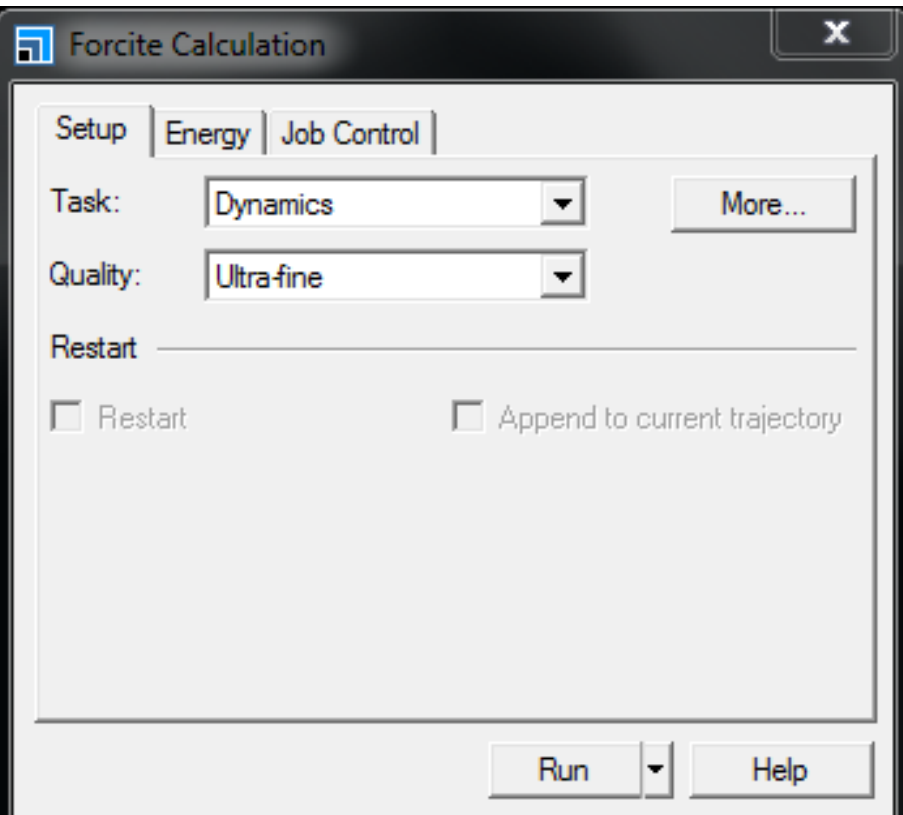
目 Forcite Dynamics

Dynamics |Thermostat | Barostat | Advanced |

Ensemble:

Initial velocities: $\quad$ Current

Temperature: $\quad \sqrt{298}$ K

Pressure: $\quad \sqrt{1.0 e-4}$ GPa

Time step: $\quad 1.0$ fs

Total simulation time: 100.0 ps

Number of steps: $\quad 100000 \div$

Frame output every: $\sqrt{1000 \div} \div$ steps 


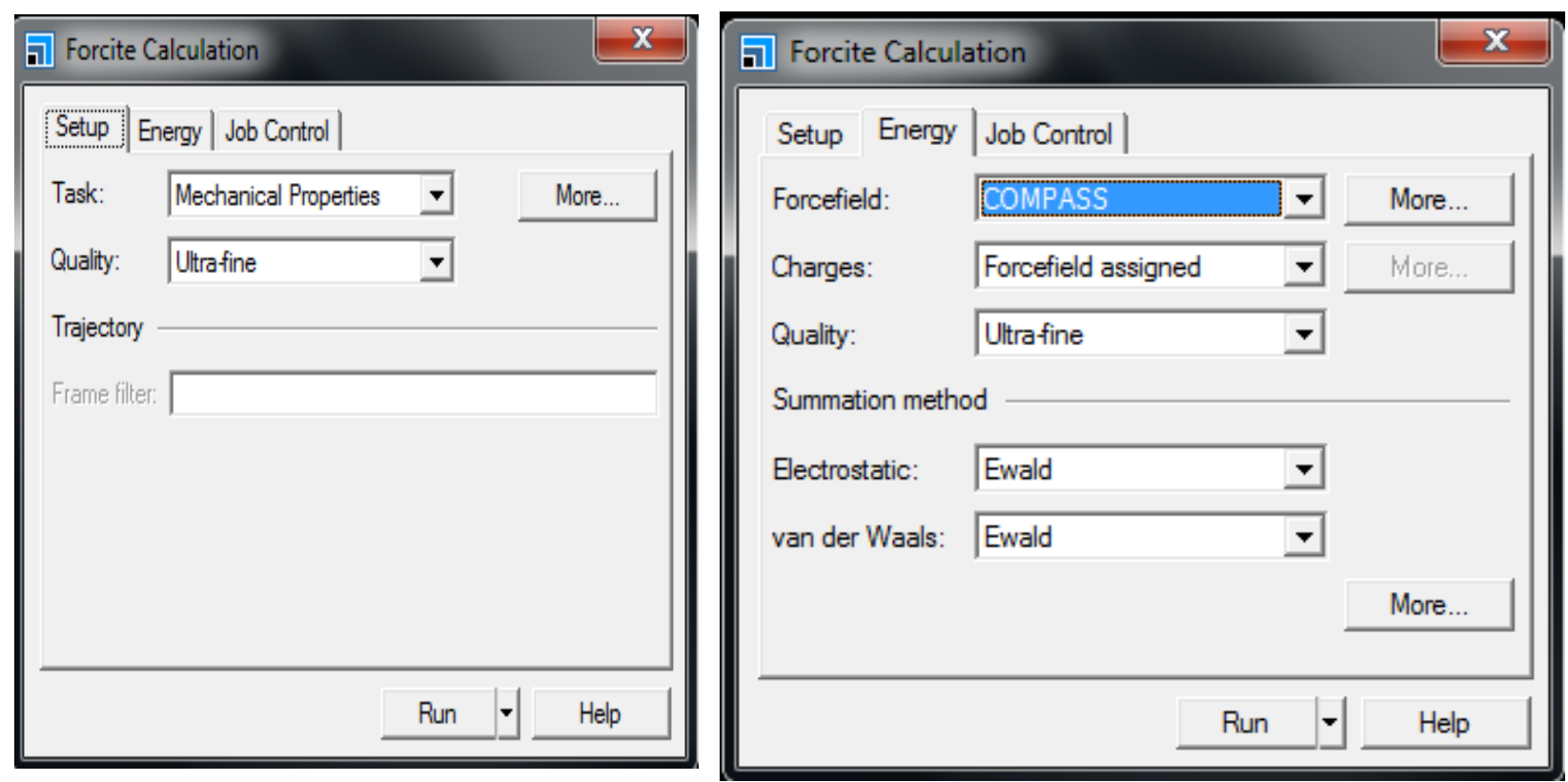

\section{V-3- Elastic properties of Portlandite}

\begin{tabular}{|llrrr|} 
Bulk modulus (GPa) & & Reuss & Voigt & Hill \\
Shear modulus (GPa) & $=$ & 11.0004 & 72.2364 & 46.2502 \\
\hline
\end{tabular}




\section{VI- Ettringite}

\section{VI-1- Geometry optimization}
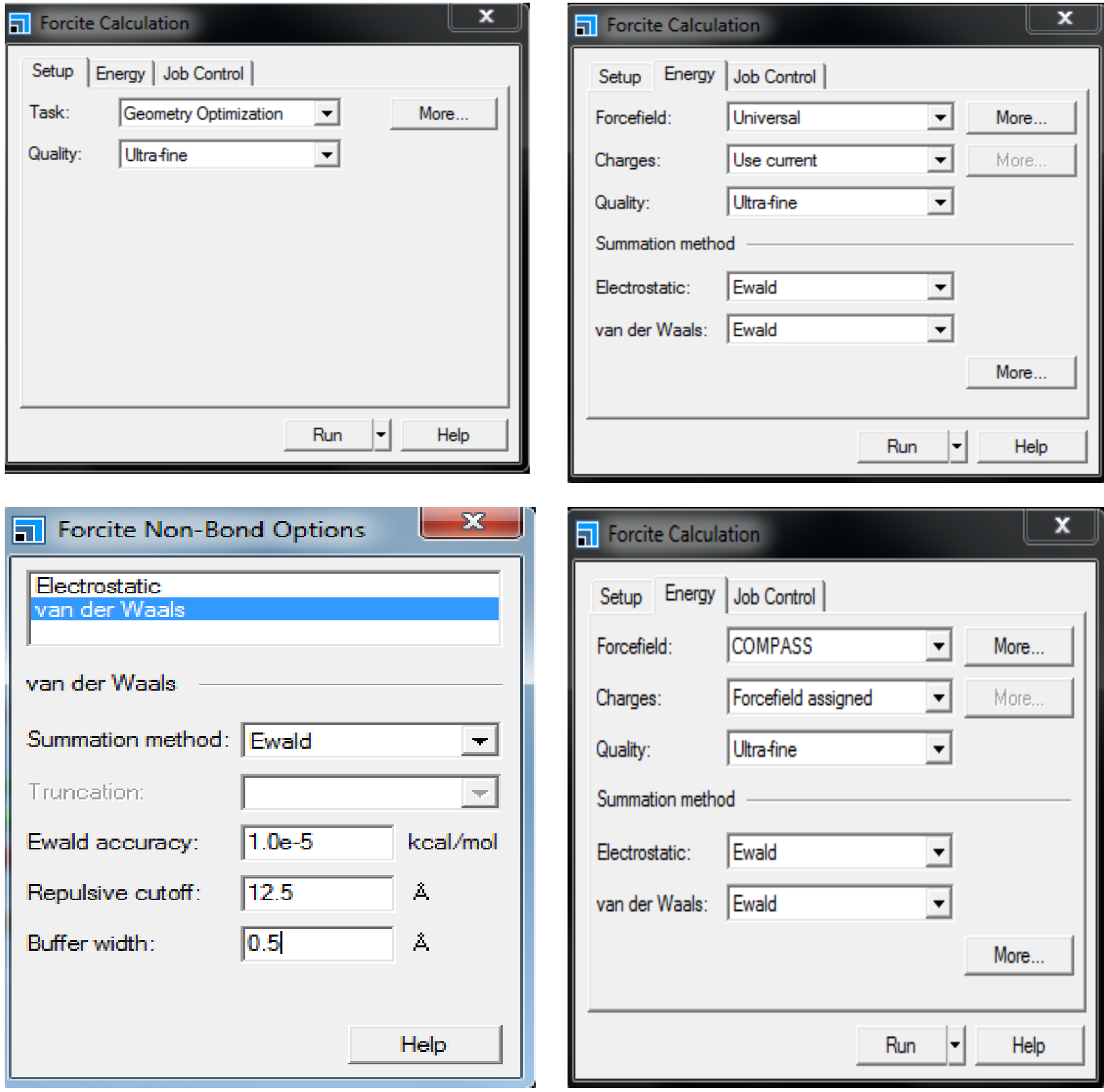


\section{VI-2- Dynamic simulation parameters}
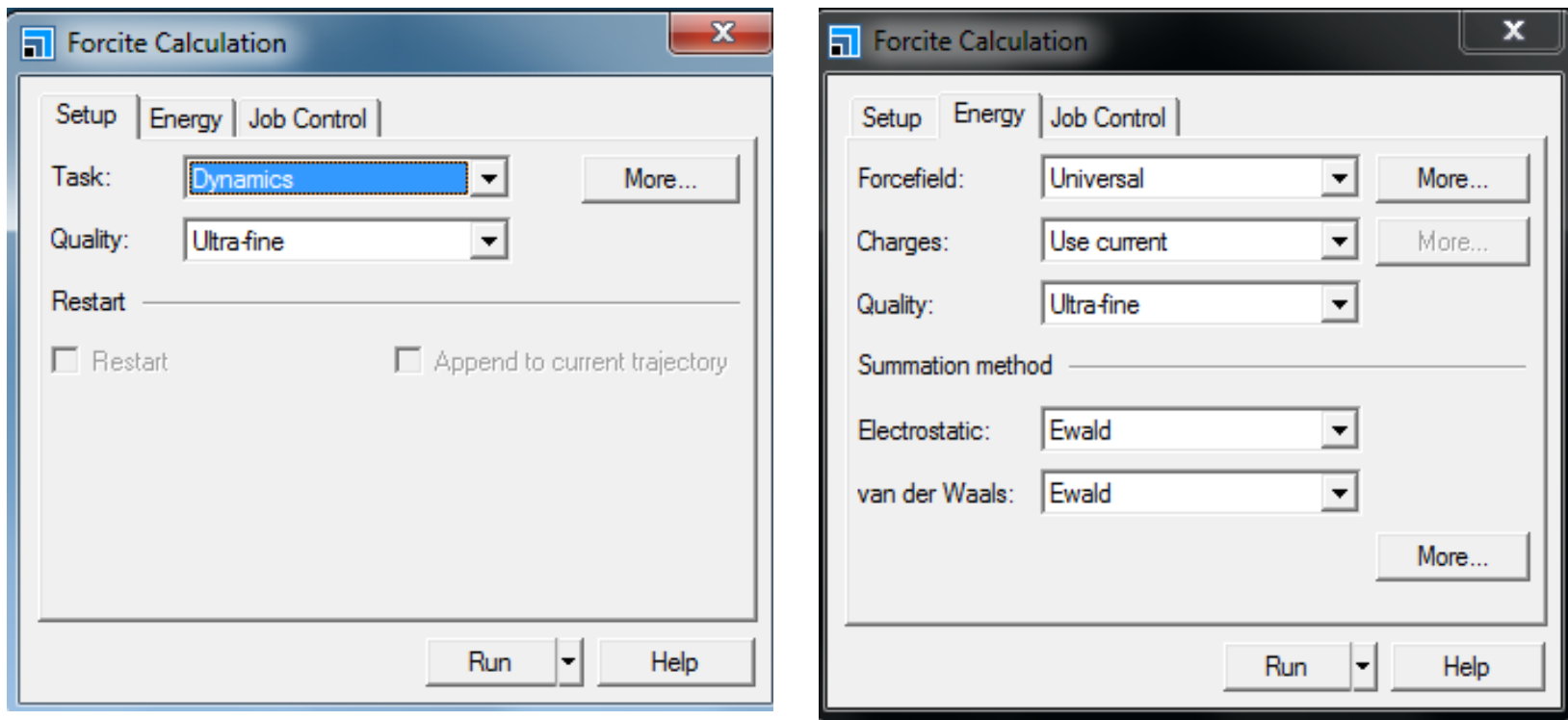

\begin{tabular}{|c|c|c|}
\hline 1] Forcite Dynamics & & $x$ \\
\hline \begin{tabular}{l|l} 
Dynamics & Thermost
\end{tabular} & tat | Barostat | & Advanced | \\
\hline Ensemble: & NPT & \\
\hline Initial velocities: & Cumrent $\quad-$ & \\
\hline Temperature: & 298.0 & K \\
\hline Pressure: & $1.0 e-4$ & $\mathrm{GPa}$ \\
\hline Time step: & 0.5 & fs \\
\hline Total simulation time: & 200.0 & ps \\
\hline Number of steps: & $400000 \div$ & \\
\hline \multirow[t]{2}{*}{ Frame output every: } & 5000 & steps \\
\hline & & Help \\
\hline
\end{tabular}

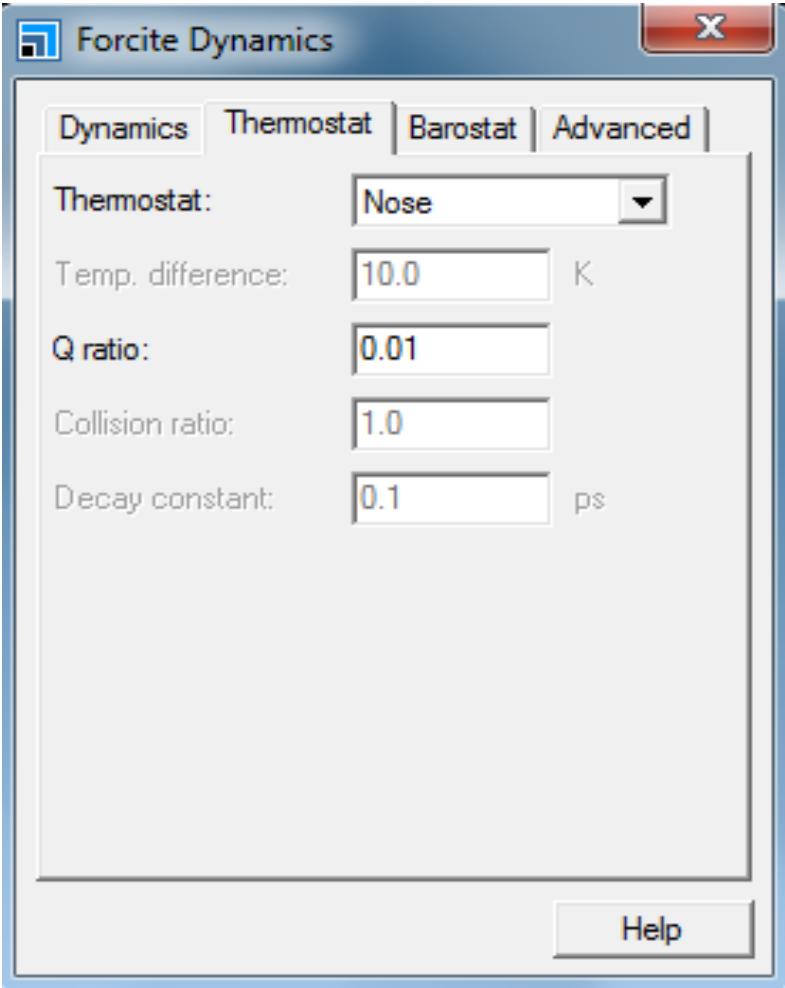



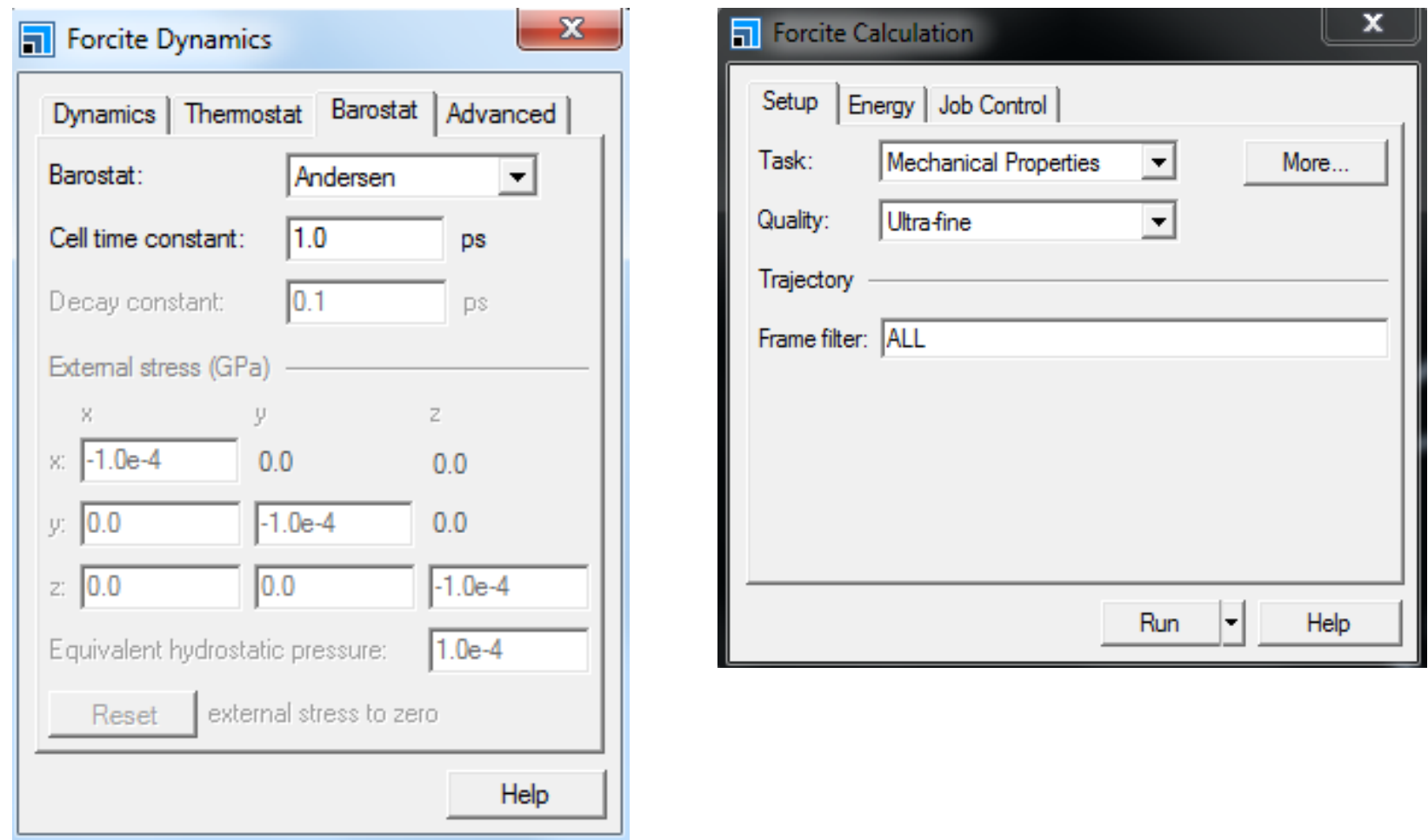

\section{VI-3- Elastic properties of Ettringite}

\begin{tabular}{|c|c|c|}
\hline $\begin{array}{l}\text { Bulk modulus (GPa) } \\
\text { shear modulus (GPa) }\end{array}$ & $\begin{array}{r}\text { Reuss } \\
14.1734 \\
6.6668\end{array}$ & $\begin{array}{r}\mathrm{Hi11} \\
15.0339 \\
7.3315\end{array}$ \\
\hline Compressibility $(1 /$ TPa $)=$ & 70.5549 & \\
\hline $\begin{array}{lc}\text { Young } & \text { Modulus (GPa) } \\
X= & 14.0316 \\
Y= & 15.7726 \\
Z= & 32.5780\end{array}$ & $\begin{array}{ll}\text { Poisson Ratios } & \\
\text { Exy }= & 0.3658 \mathrm{Exz}= \\
\text { Eyx }= & 0.4112 \mathrm{Eyz}= \\
\text { EXX }= & 0.2834 \mathrm{EZy}=\end{array}$ & $\begin{array}{l}0.1220 \\
0.1993 \\
0.4117\end{array}$ \\
\hline
\end{tabular}




\section{VII- Tobermorite (9A)}

\section{VII-1- Geometry optimization}
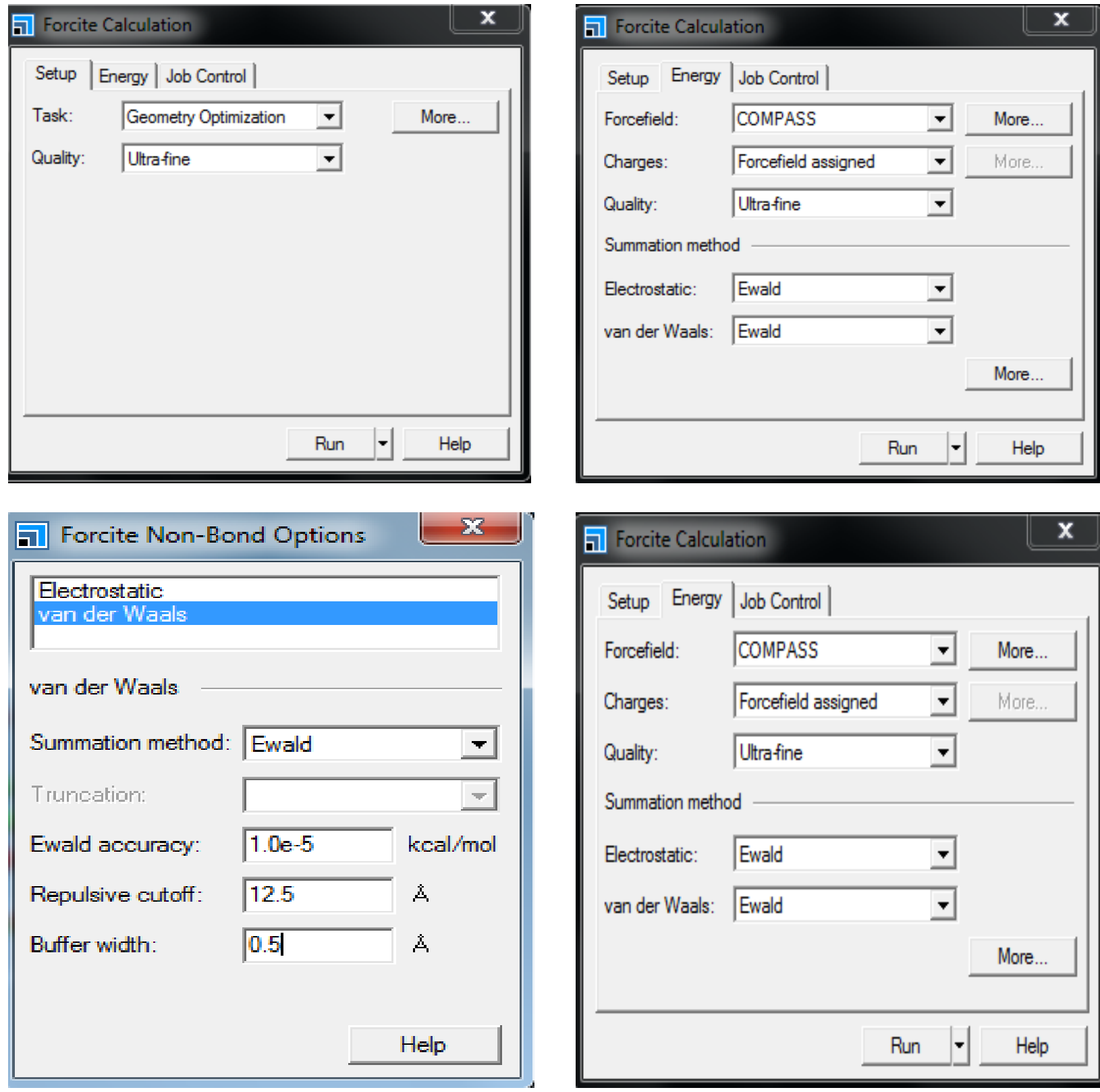

\begin{tabular}{|c|c|c|c|}
\hline \multirow{2}{*}{$\begin{array}{l}\text { Setup Energy } \\
\text { Forcefield: }\end{array}$} & \multicolumn{3}{|l|}{ Job Control | } \\
\hline & COMPASS & 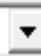 & More... \\
\hline Charges: & Forcefield assigned & 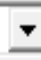 & More... \\
\hline Quality: & Ultrafine & 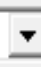 & \\
\hline \multicolumn{4}{|c|}{ Summation method } \\
\hline Electrostatic: & Ewald & 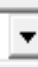 & \\
\hline \multirow[t]{3}{*}{ van der Waals: } & Ewald & 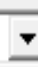 & \\
\hline & & & More... \\
\hline & & & Help \\
\hline
\end{tabular}

\section{目 Forcite Calculation}




\section{VII-2- Dynamic simulation}
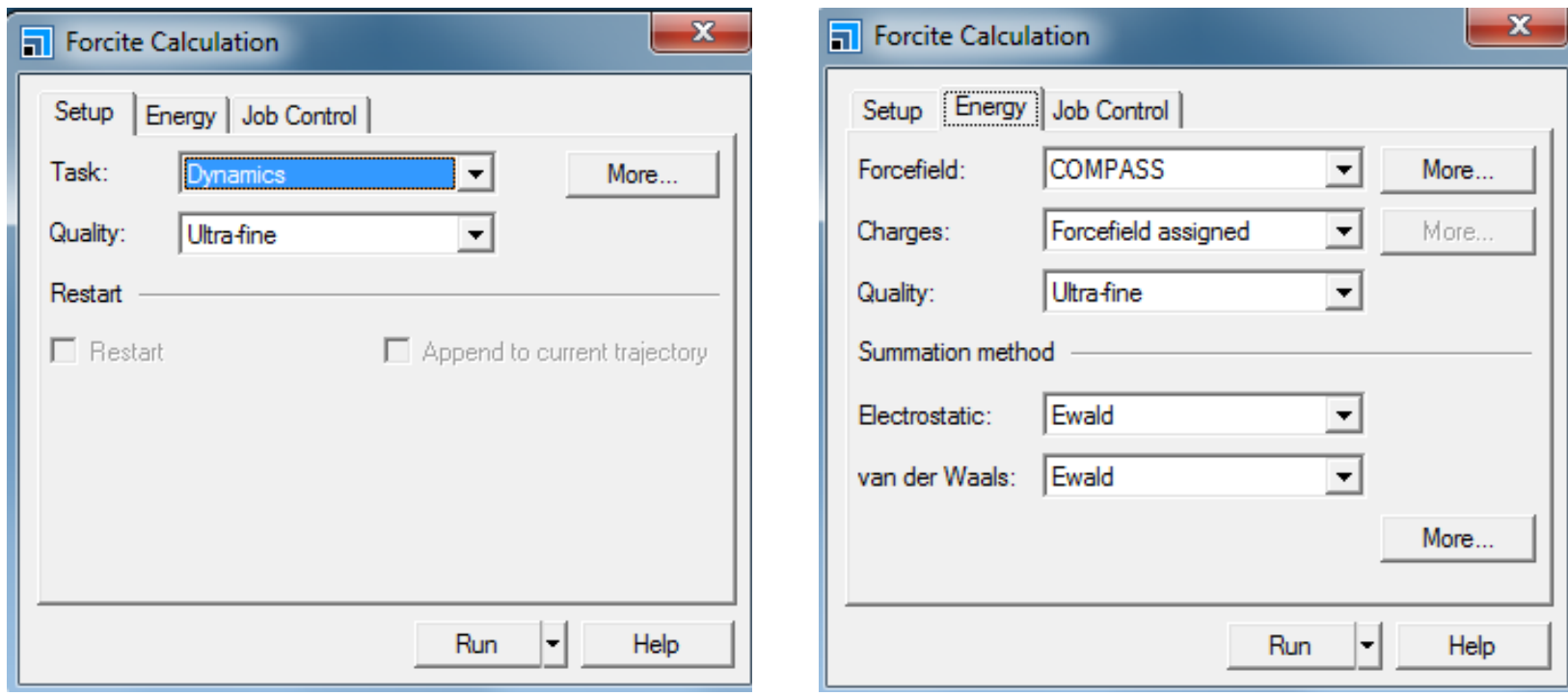

\begin{tabular}{|c|c|c|c|}
\hline Forcite Dynamics & & & $\mathbf{x}$ \\
\hline Dynamics $\mid$ Thermost & at | Barosta & at $\mid A$ & Advanced | \\
\hline Ensemble: & NPT & $\nabla$ & \\
\hline Initial velocities: & Current & $\nabla$ & \\
\hline Temperature: & 298.0 & & $\mathrm{~K}$ \\
\hline Pressure: & 1.0e-4 & & GPa \\
\hline Time step: & 1.0 & & fs \\
\hline Total simulation time: & 500.0 & & ps \\
\hline Number of steps: & 500000 & $\div$ & \\
\hline Frame output every: & 5000 & $\div$ & steps \\
\hline & & & Help \\
\hline
\end{tabular}

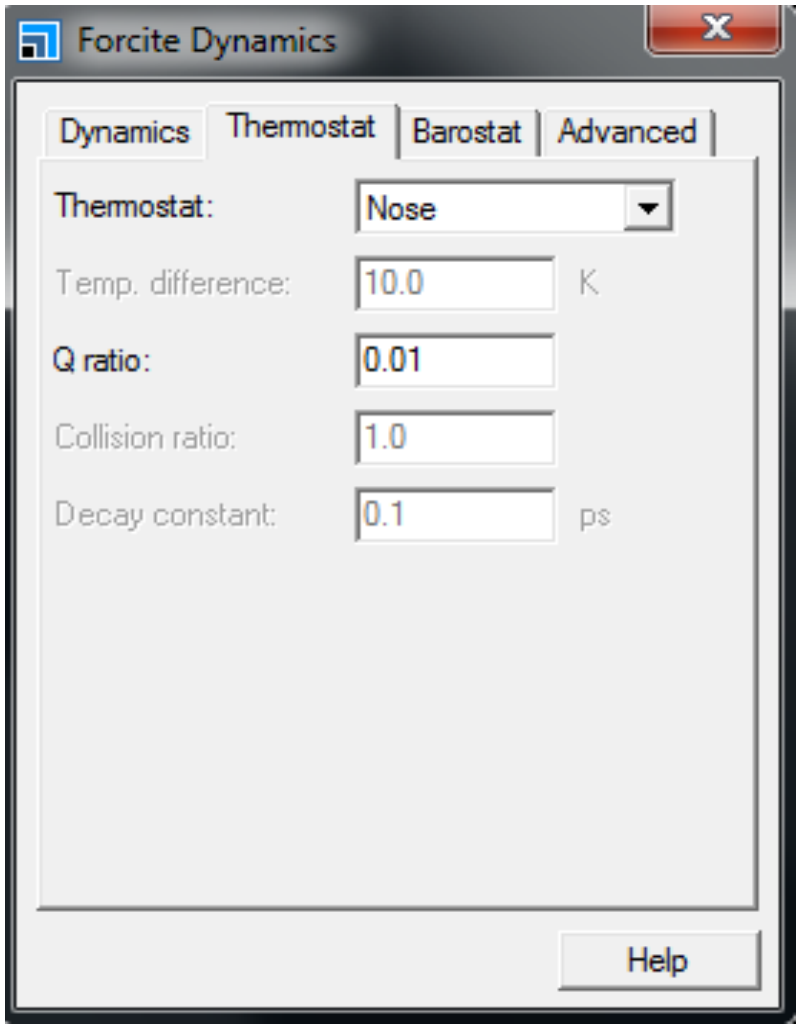



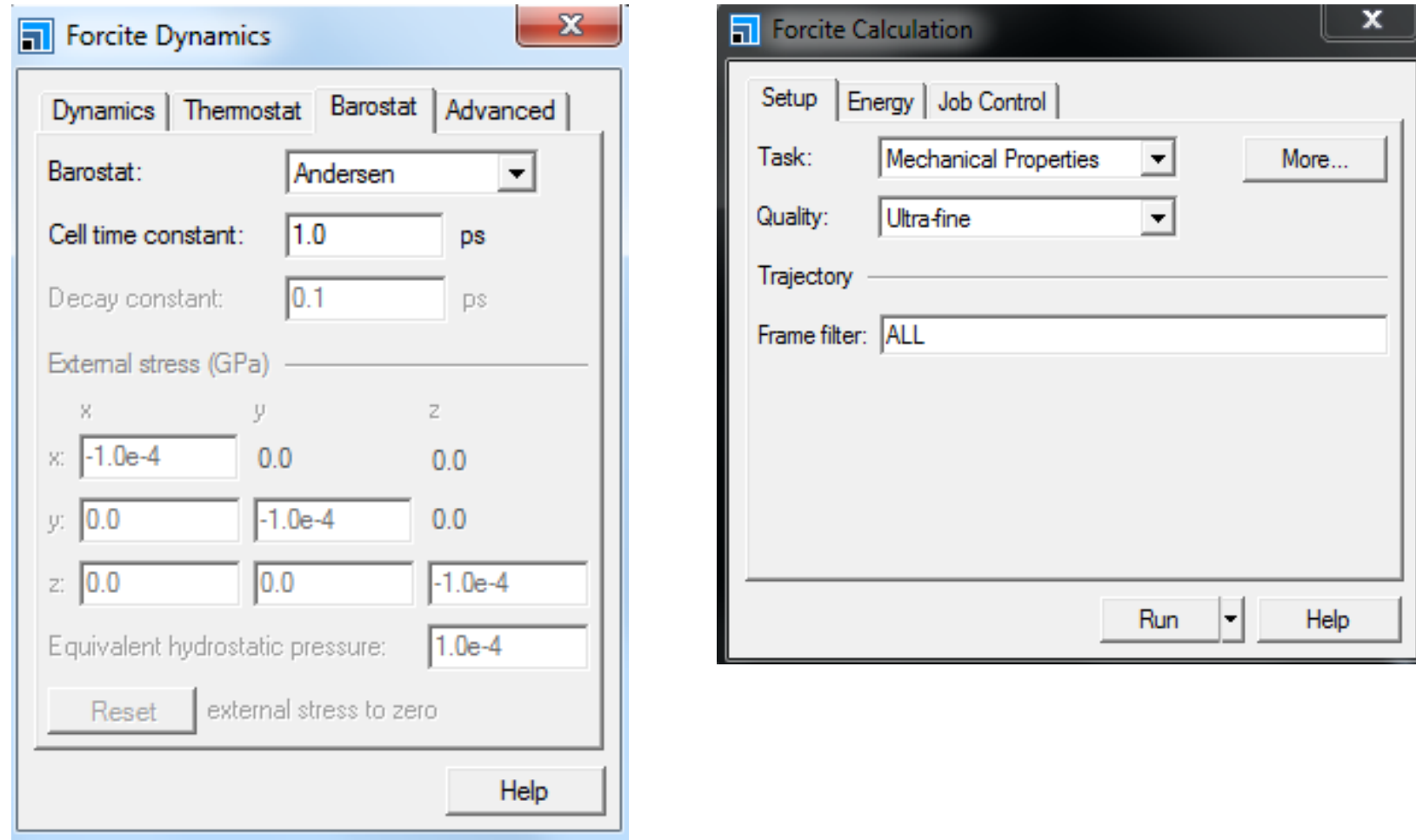

VII-3- Elastic properties of Tobermorite 9A

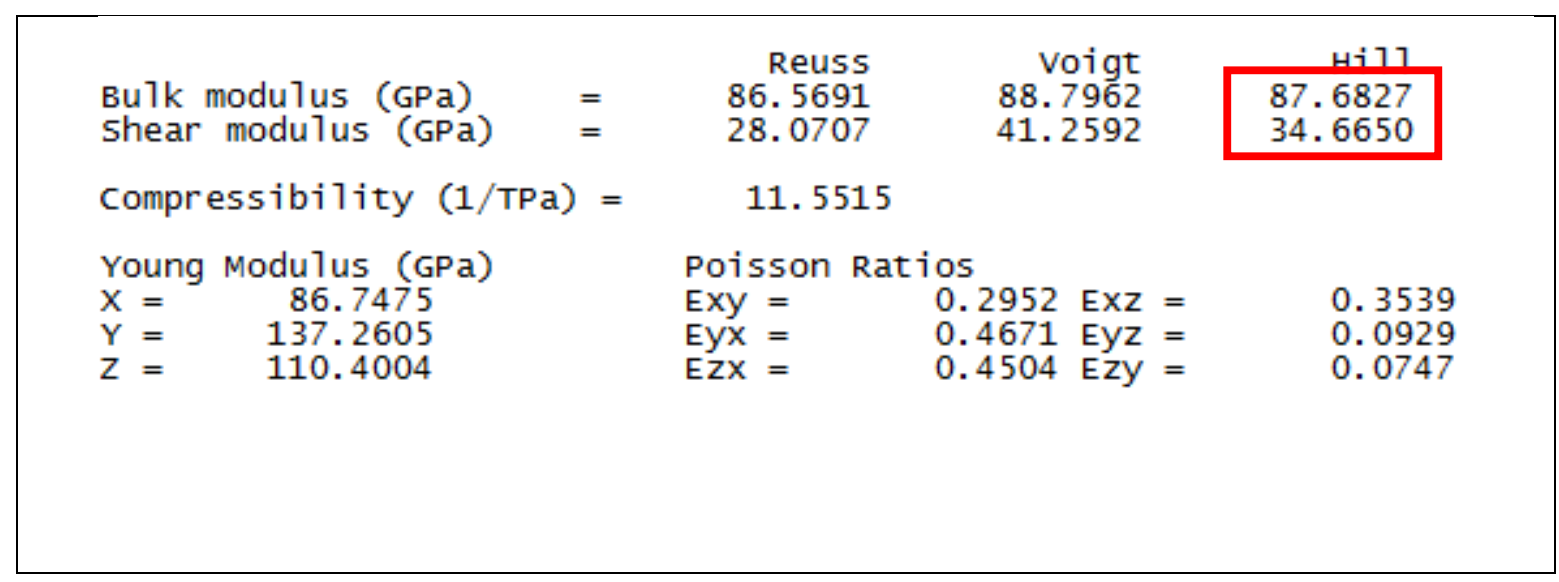


VIII- Tobermorite 11A

\section{VIII - 1- Geometry optimization}
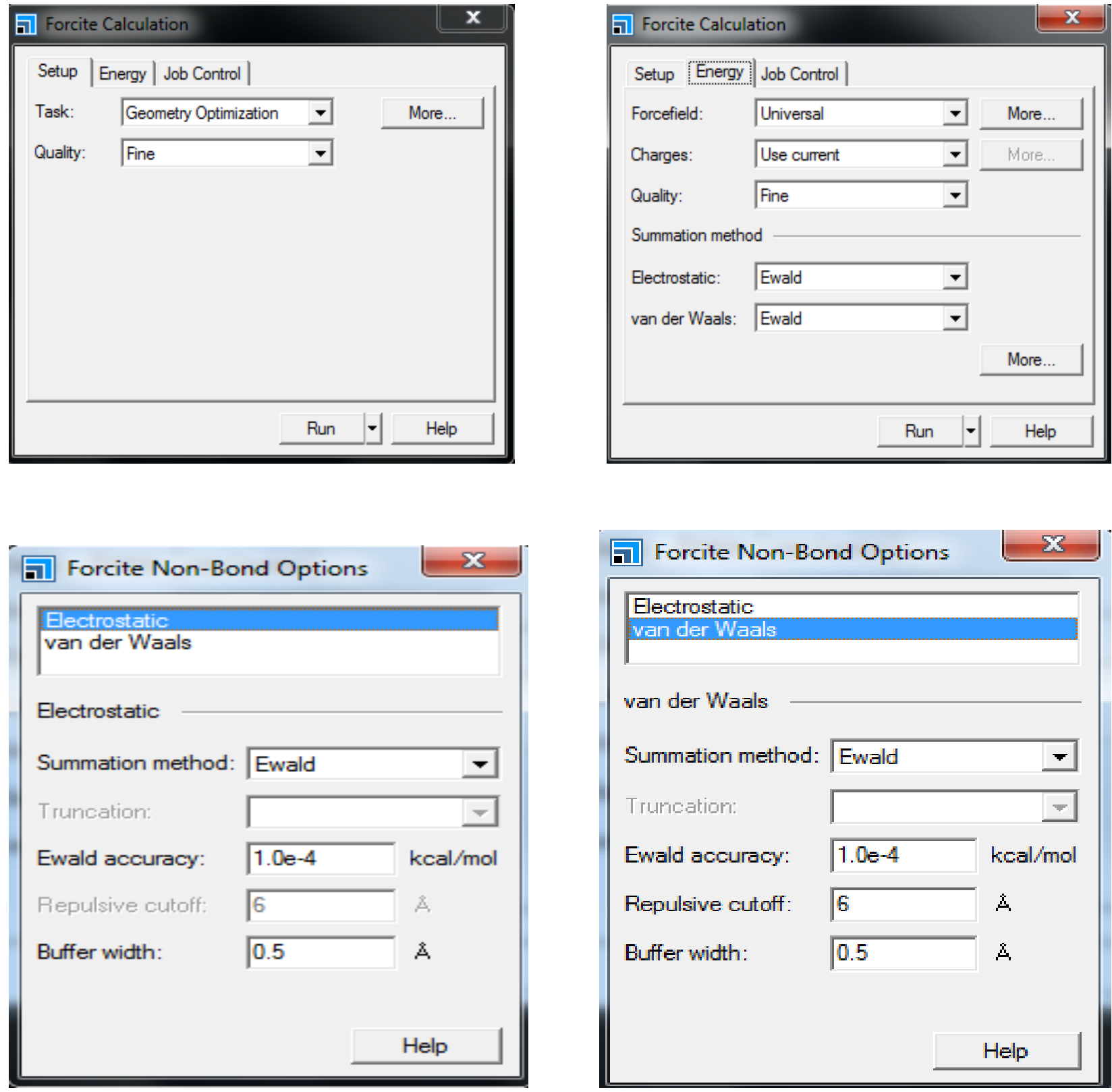

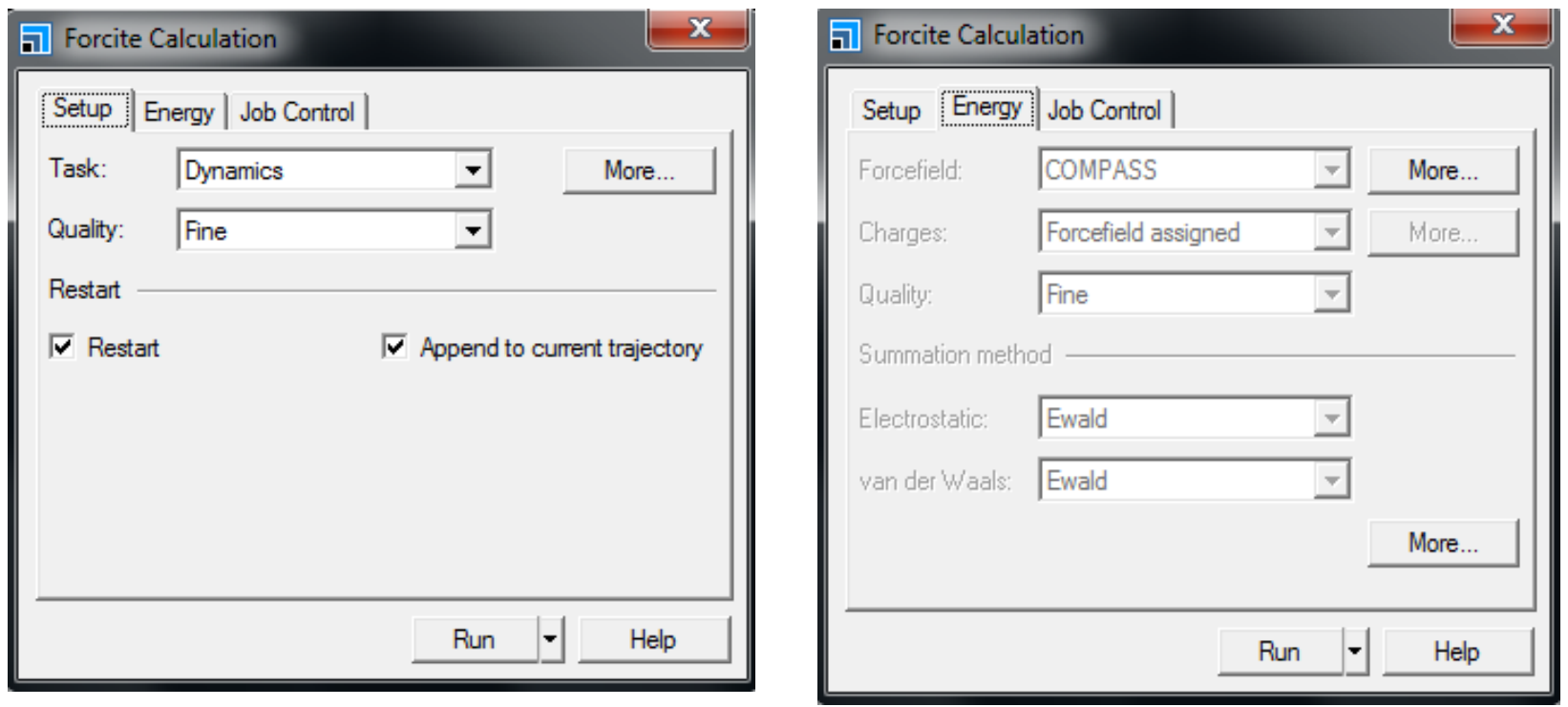

\begin{tabular}{|c|c|c|c|}
\hline 目 Forcite Dynamics & & & 2 \\
\hline \begin{tabular}{l|l} 
Dynamics & Thermost
\end{tabular} & at | Barost & at & Advanced \\
\hline Ensemble: & NPT & $\nabla$ & \\
\hline Initial velocities: & Current & $\nabla$ & \\
\hline Temperature: & 298.0 & & K \\
\hline Pressure: & $1.0 e-4$ & & GPa \\
\hline Time step: & 0.5 & & fs \\
\hline Total simulation time: & 200.0 & & ps \\
\hline Number of steps: & 400000 & $\div$ & \\
\hline Frame output every: & 5000 & $\div$ & steps \\
\hline & & & Help \\
\hline
\end{tabular}

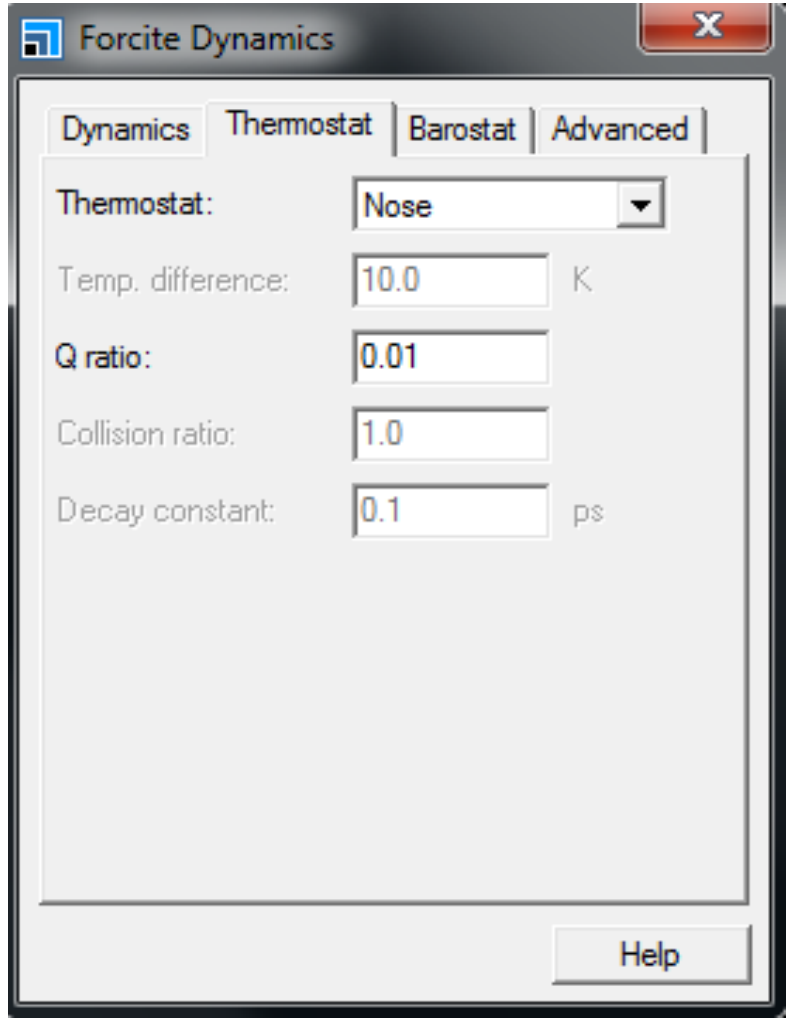



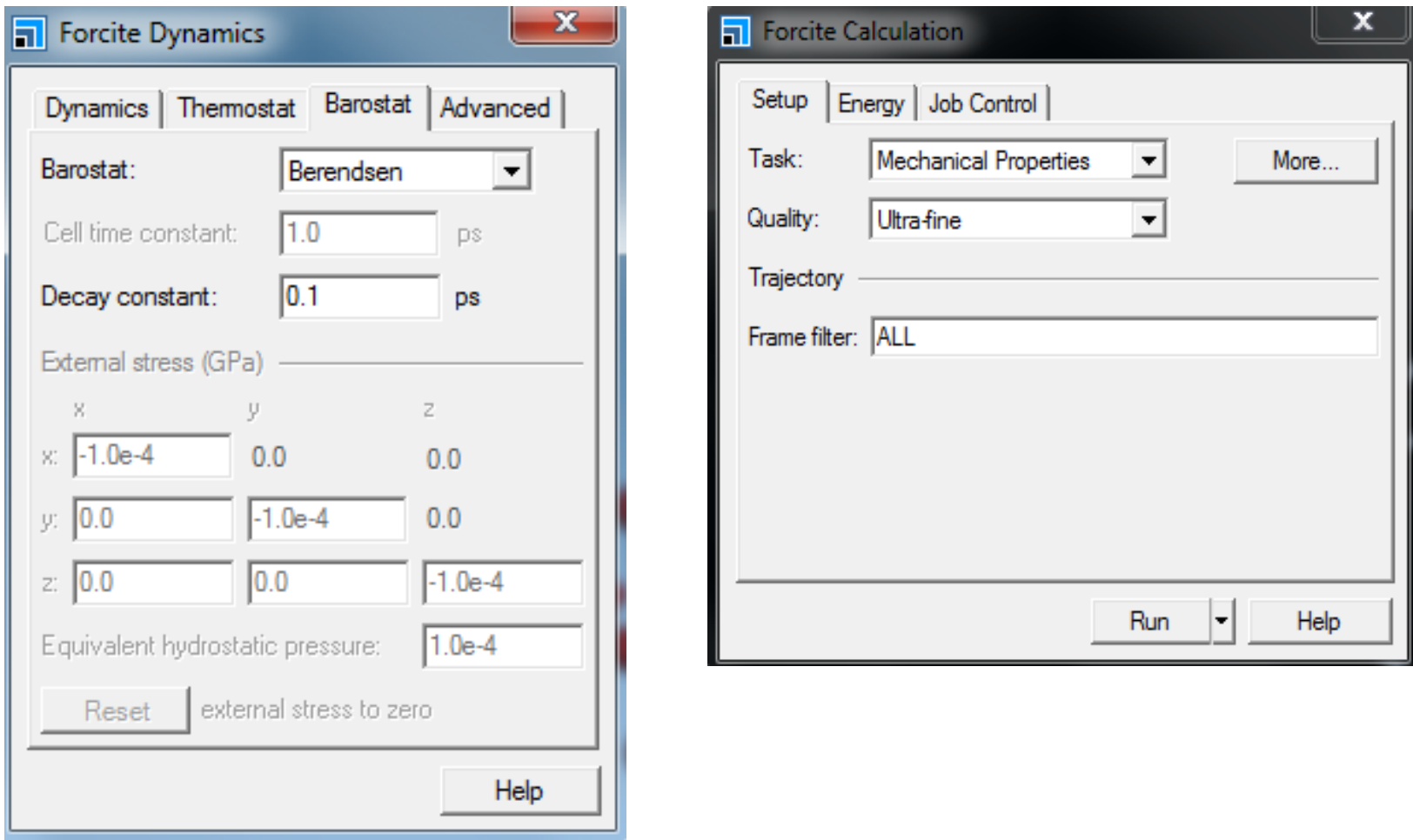

VIII -3- Results: Tobermorite 11A

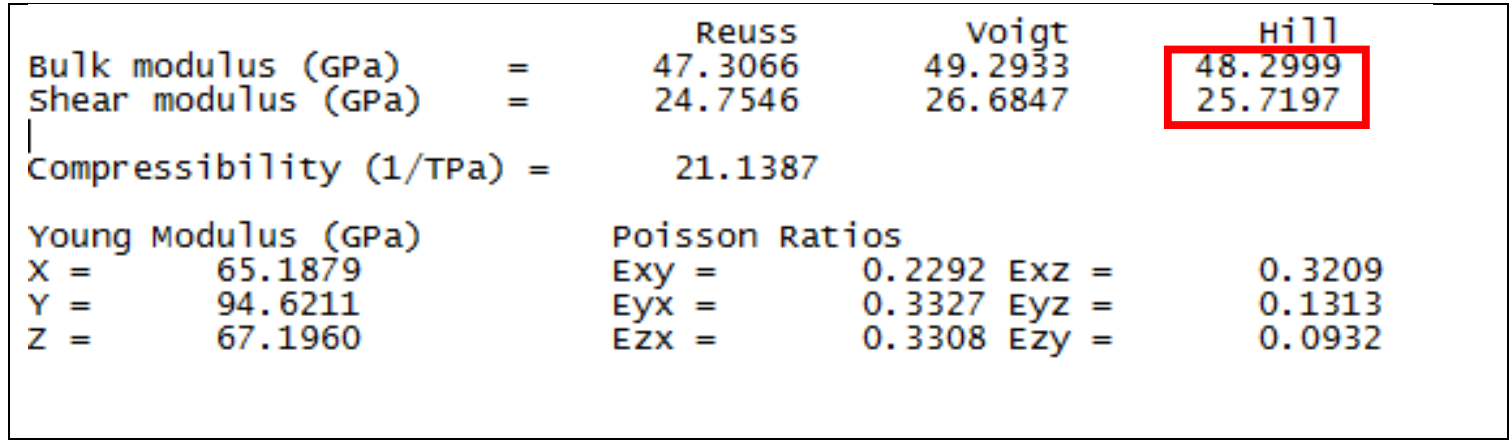




\section{IX- Tobermorite 14A}

\section{IX-1- Geometry optimization}
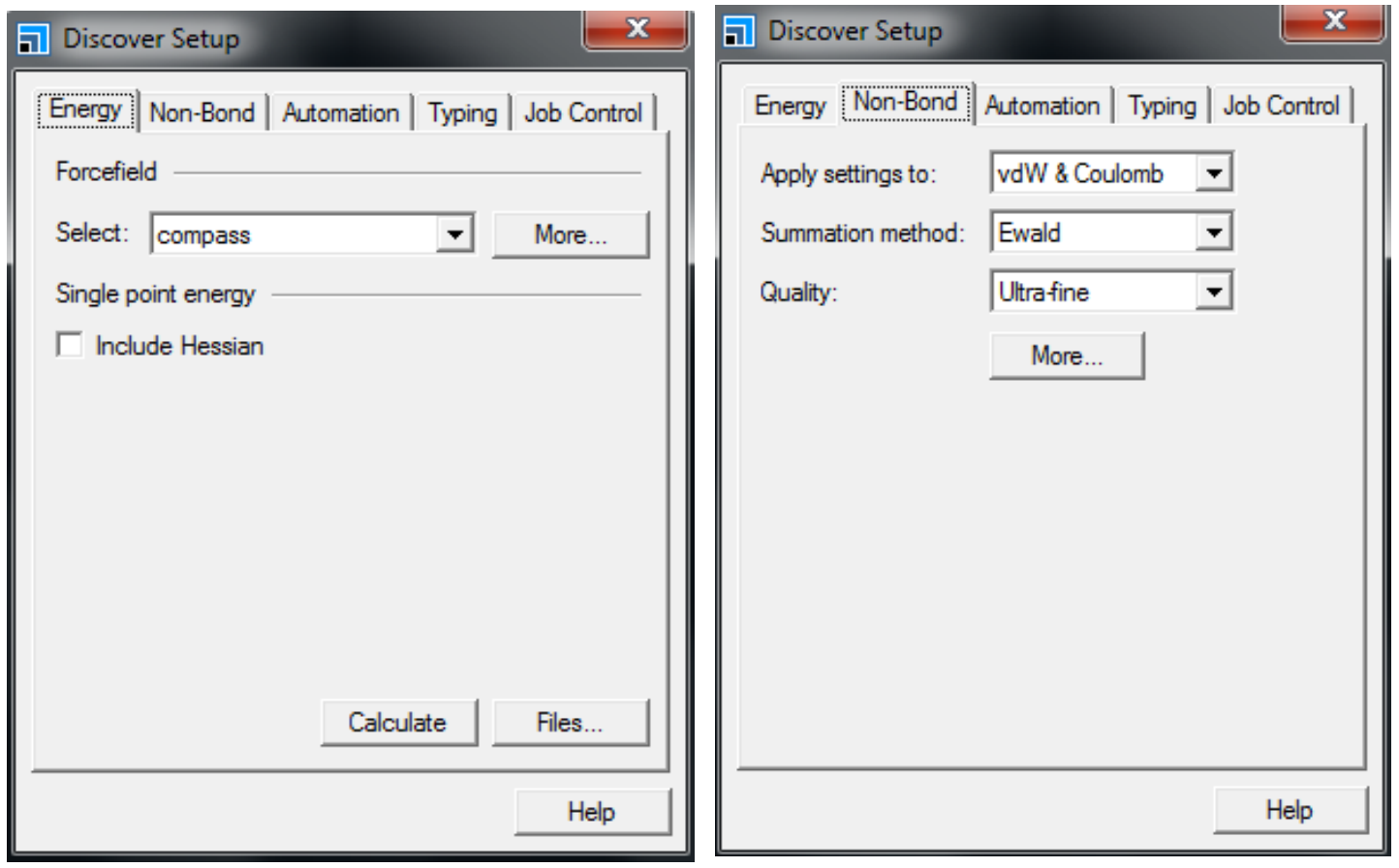

\section{IX-2- Dynamic simulation}
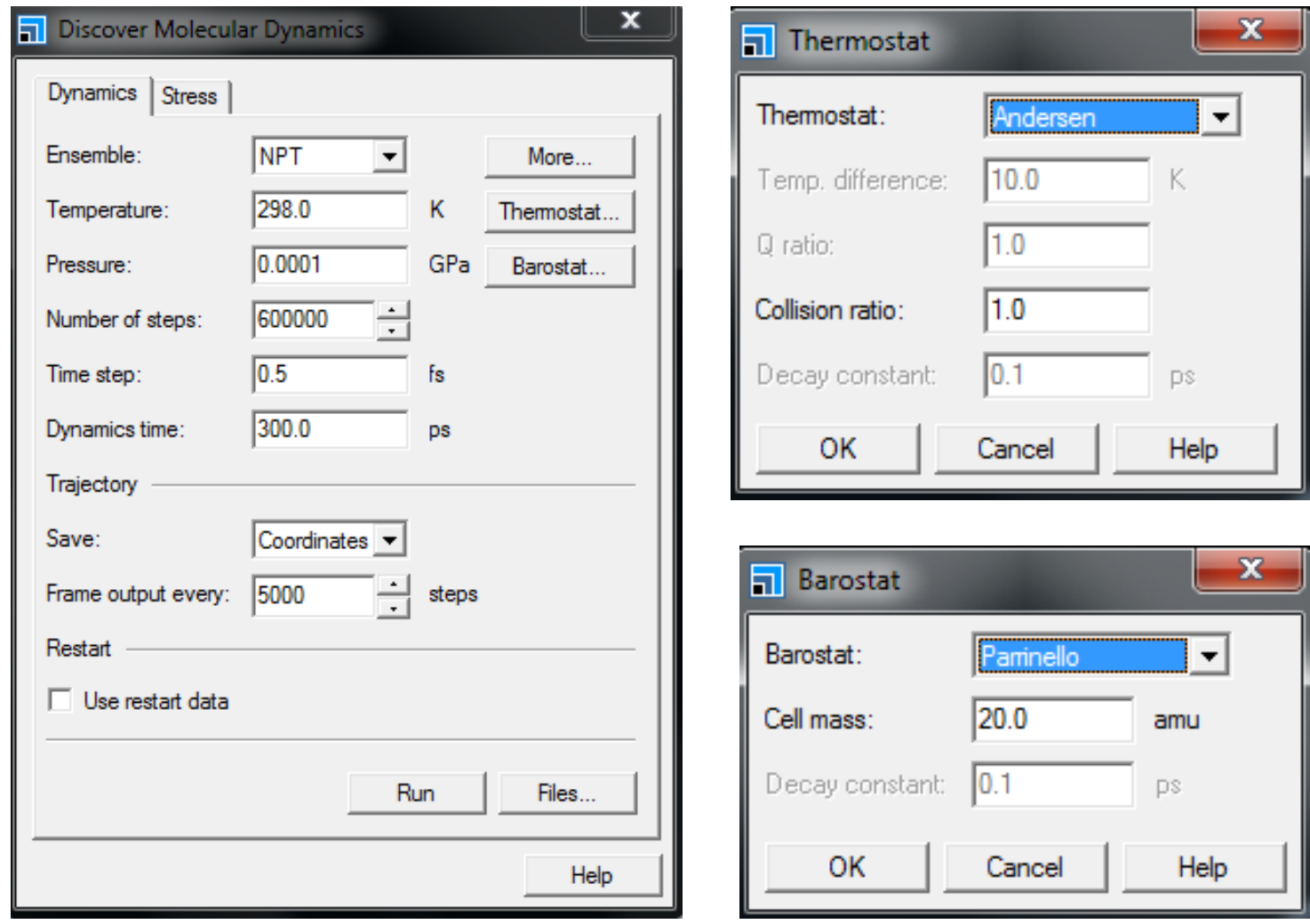

IX-3- Results (Tobermorite 14A) 


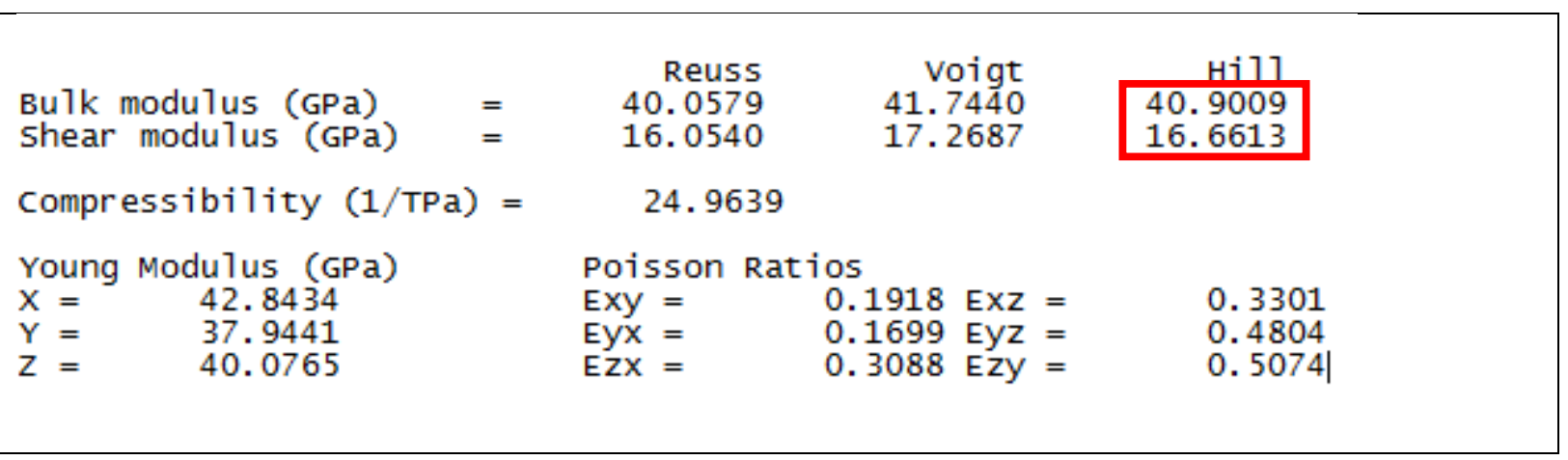

\section{X- Jennite}

\section{X-1- Geometry optimization}
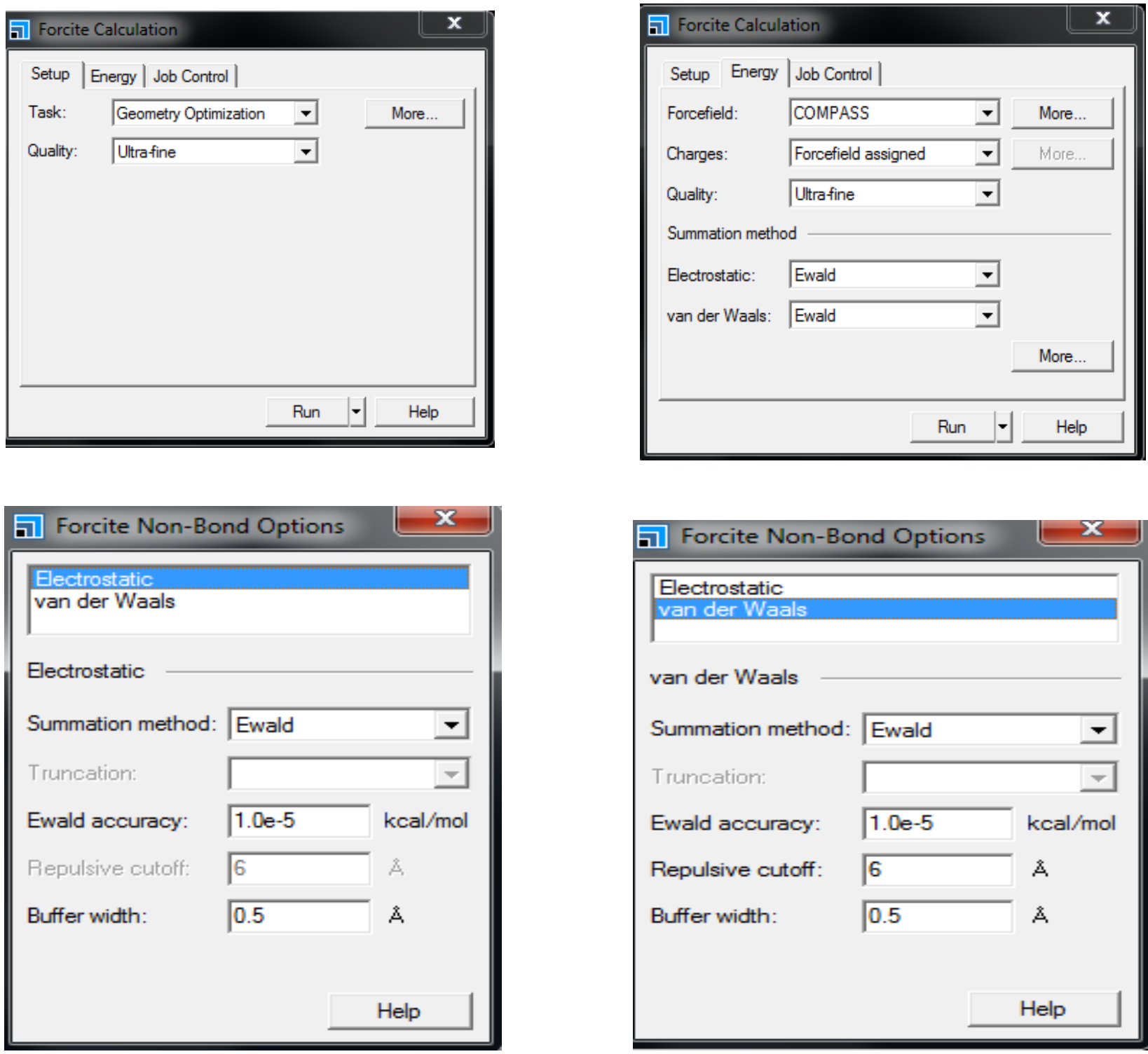


\section{X-2- Dynamic simulation}
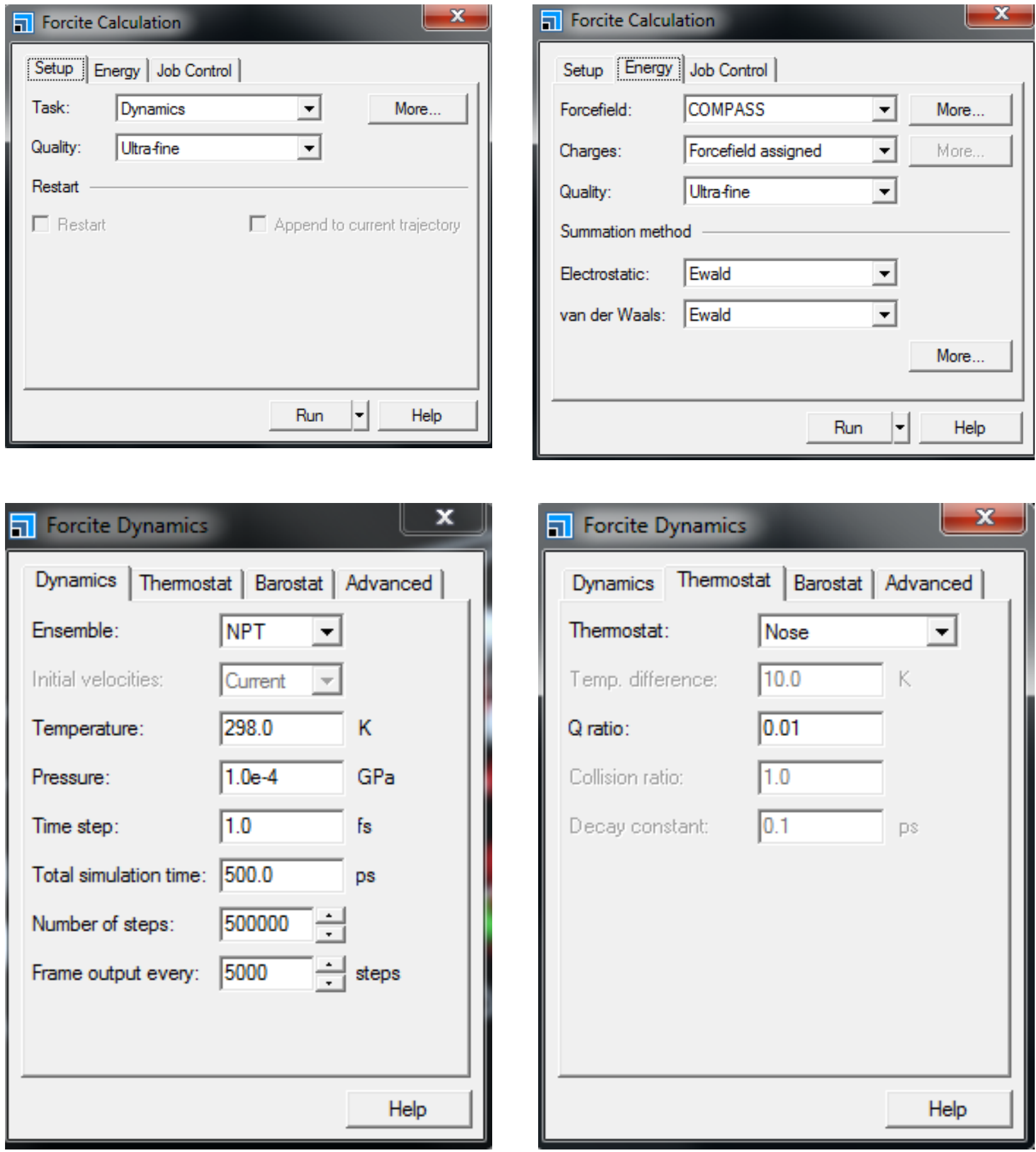

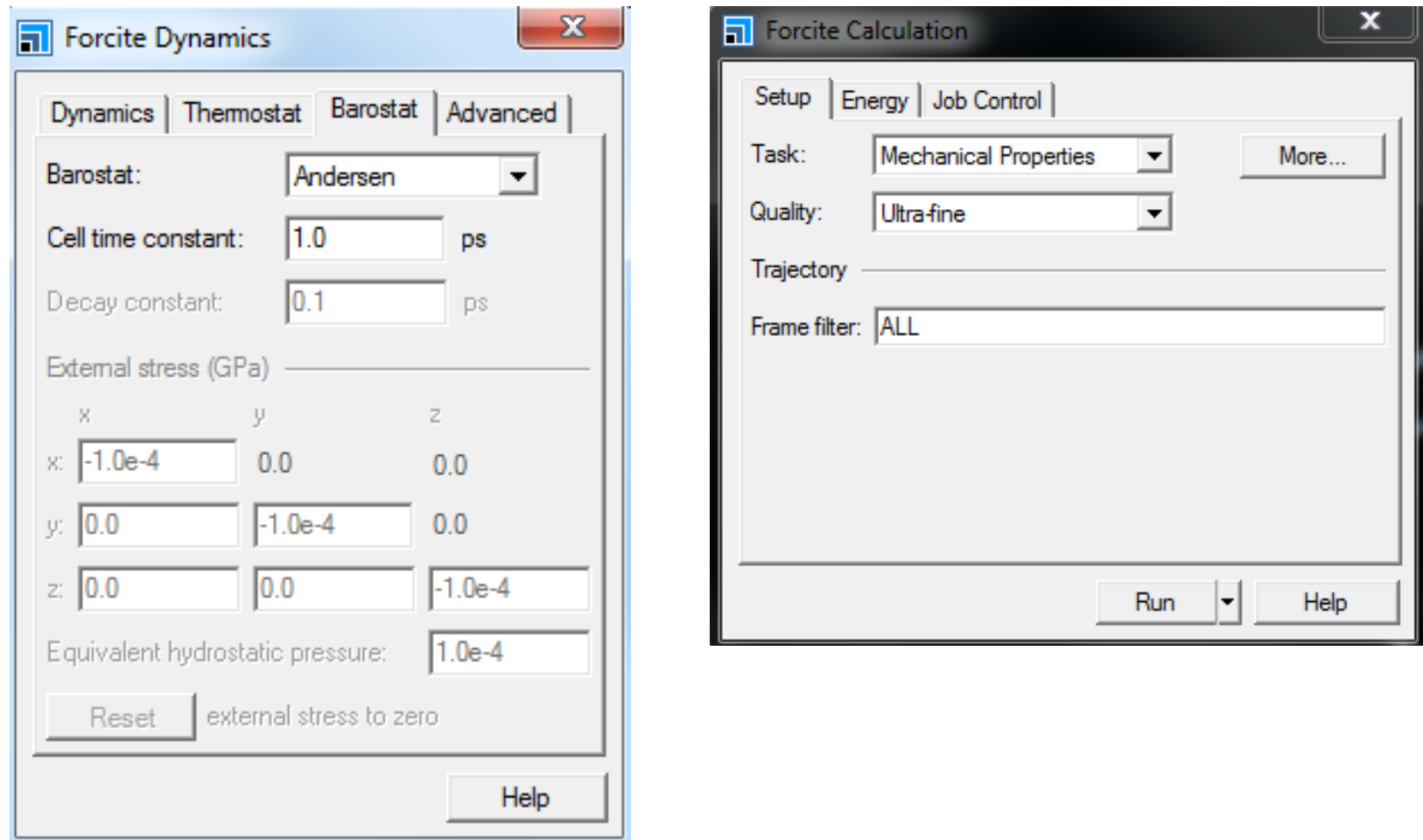

\section{X-3- Results (Jennite)}

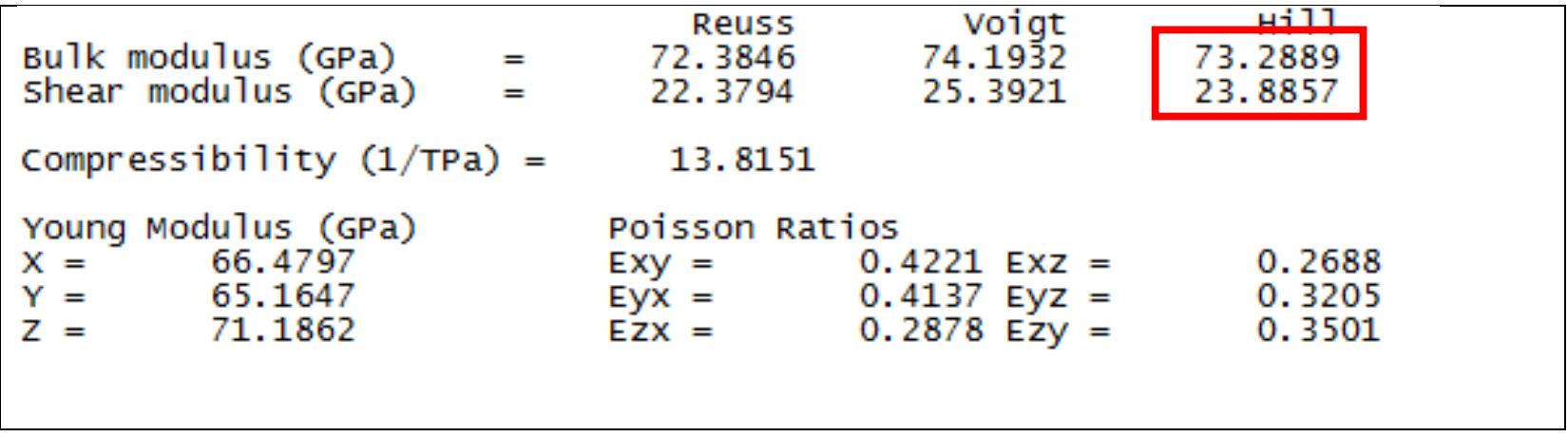




\section{XI- Tobermorite 14A (T2 dimer)}

\section{XI-1- Geometry optimization}
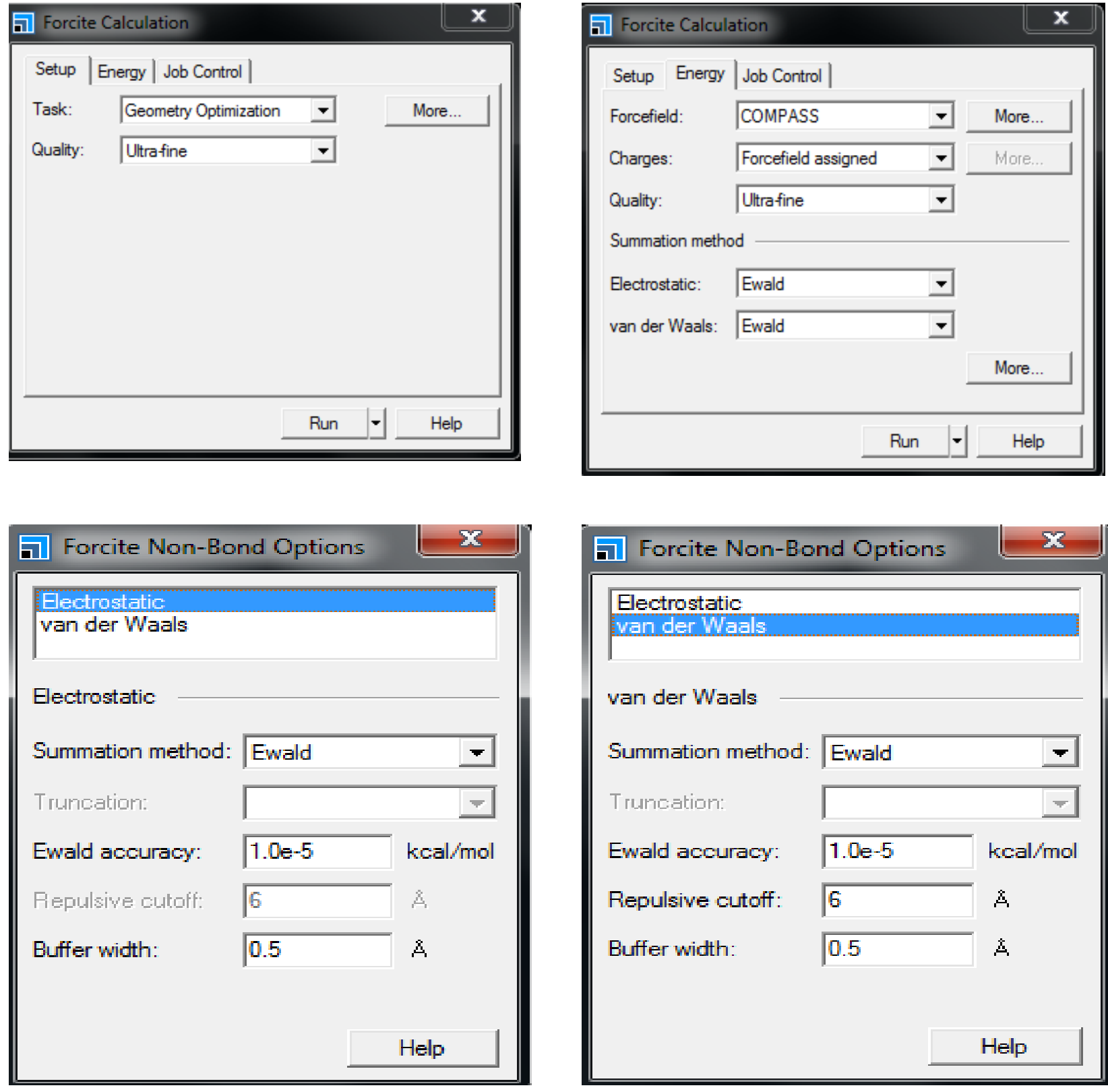


\section{XIV-2- Dynamic simulation (T2)}
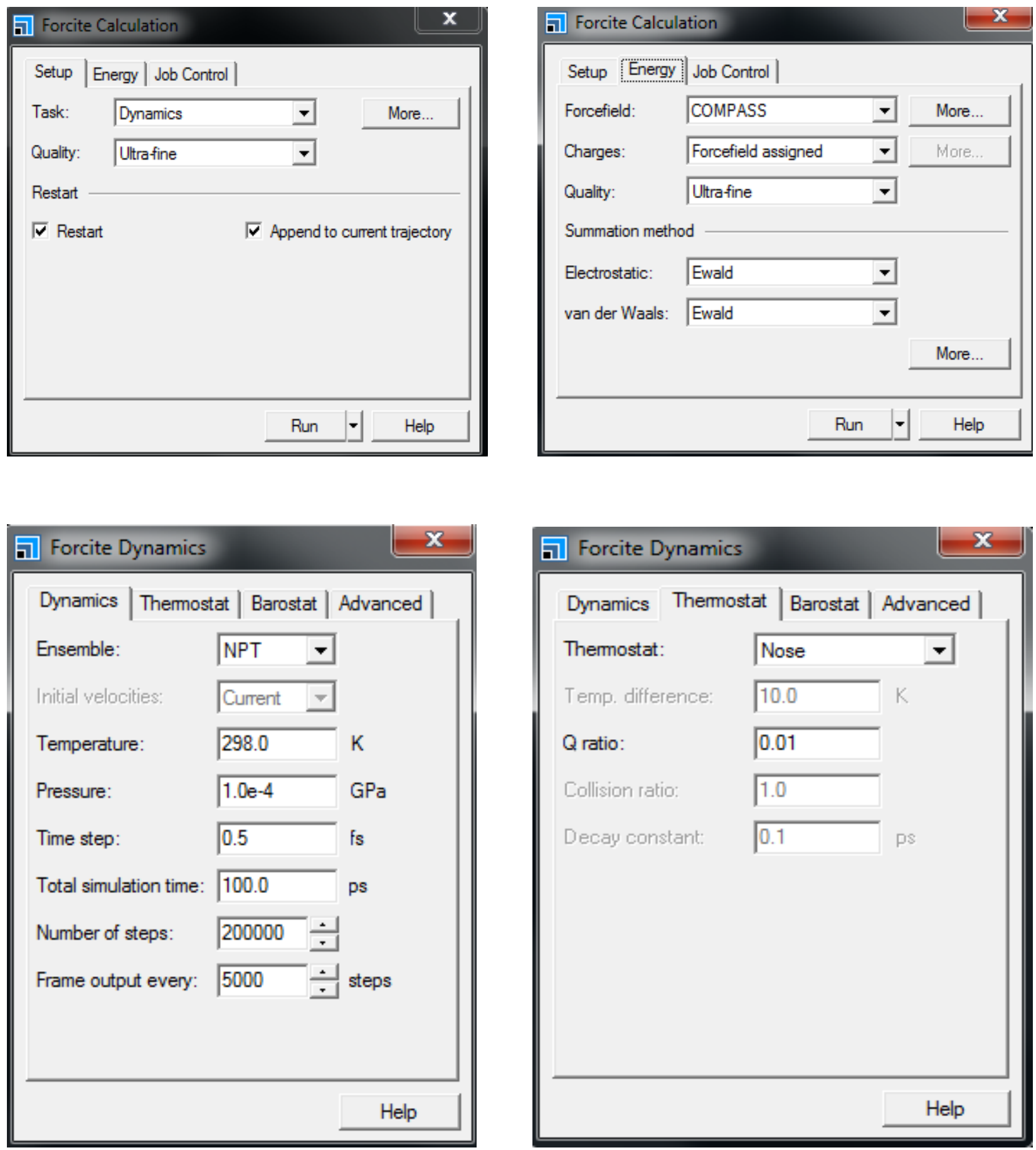

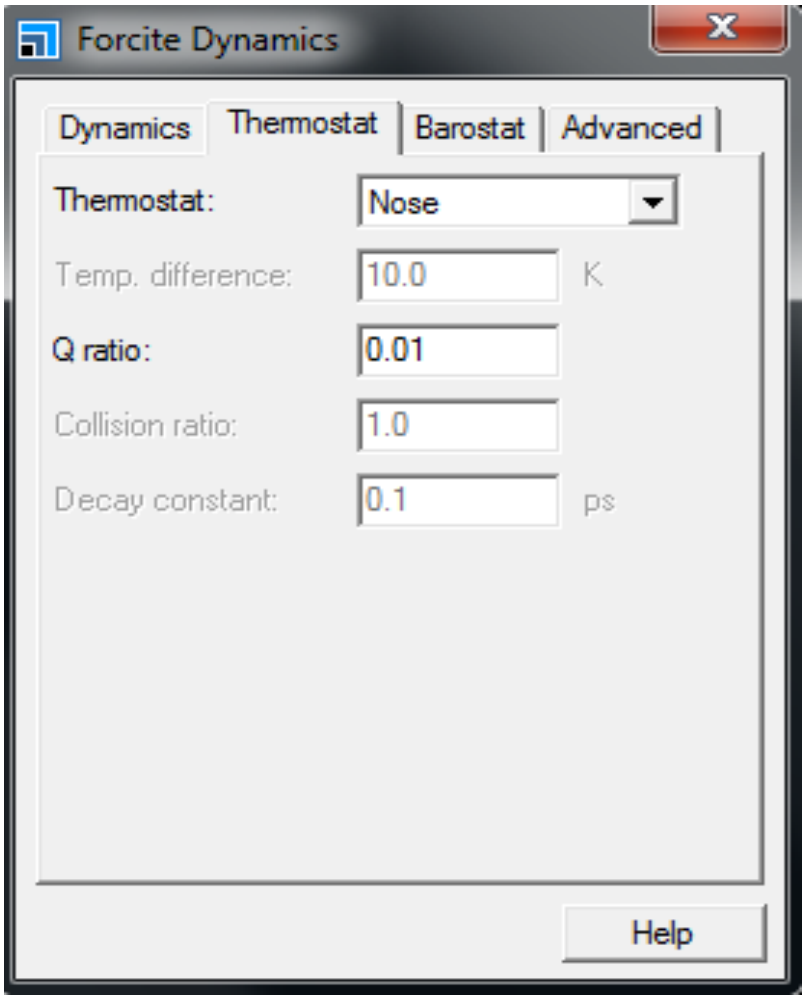



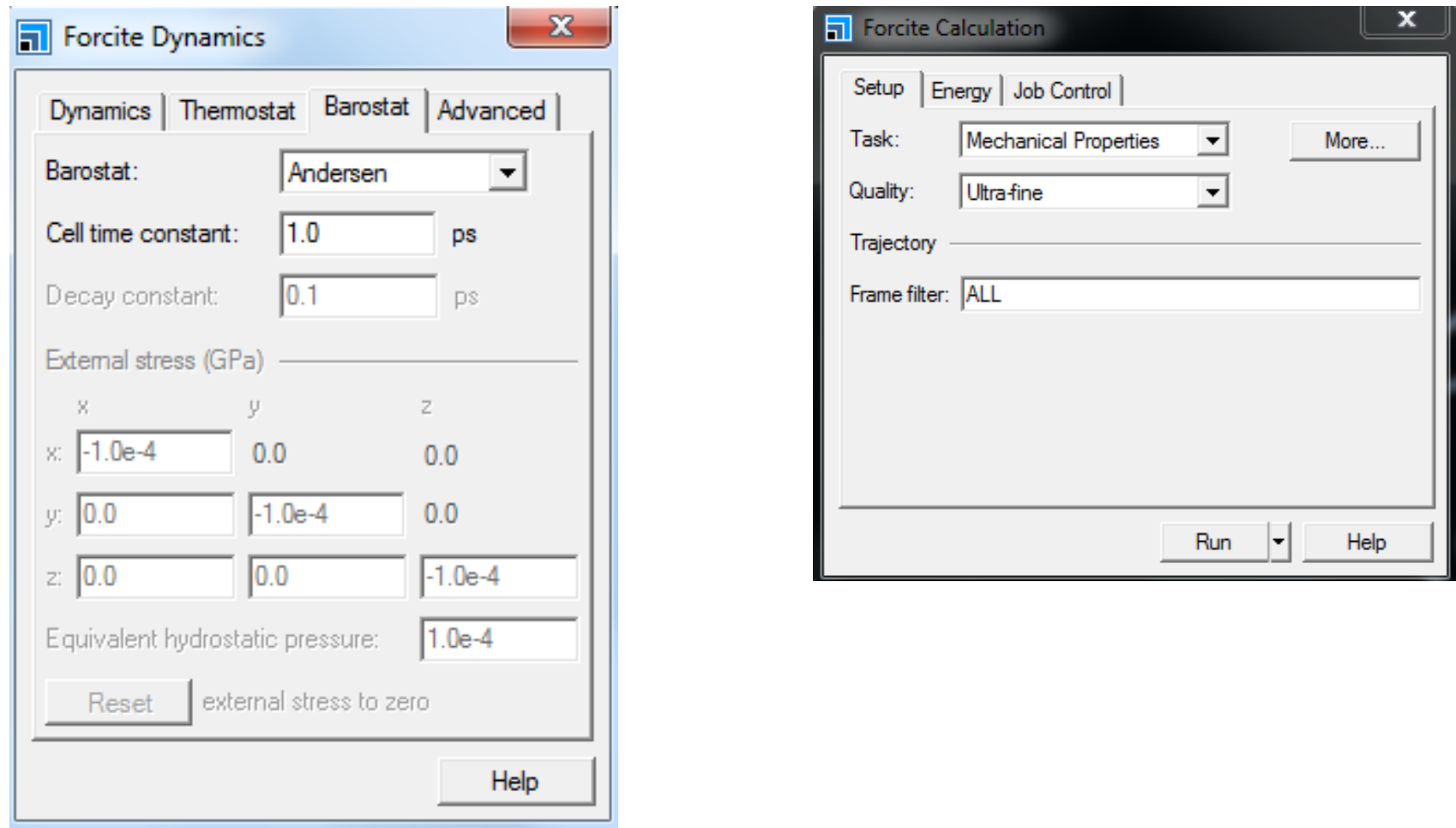

\section{XIV-3- Results (T2)}

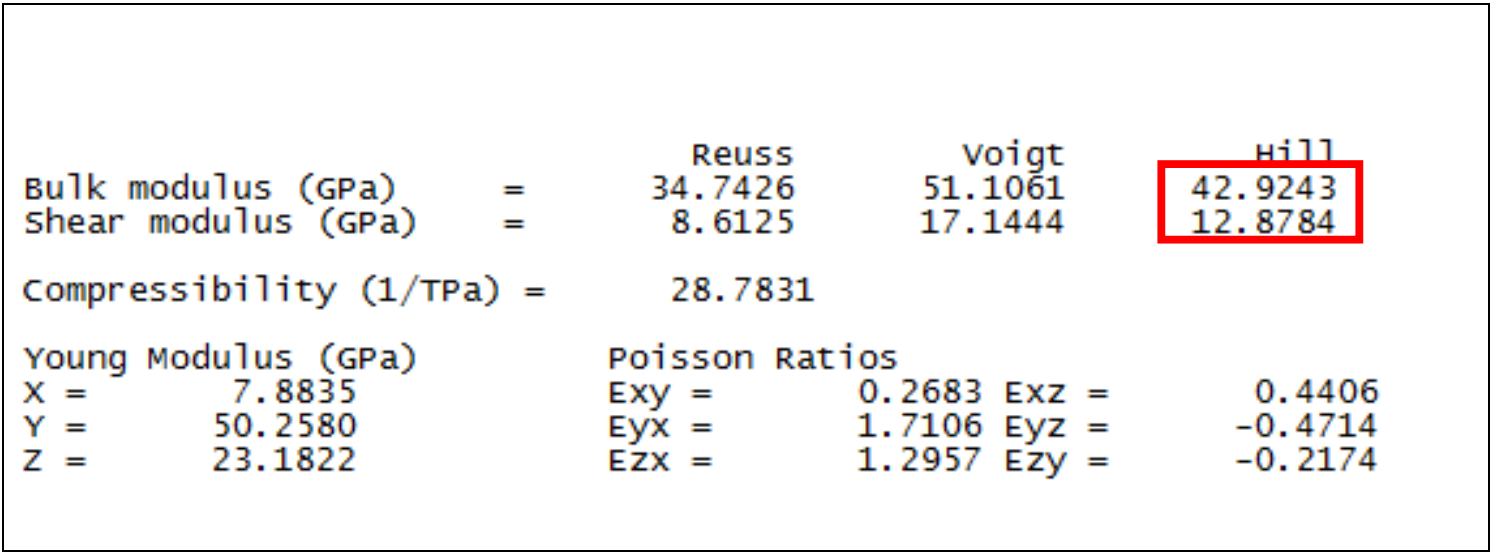




\section{XV- Jennite modified (J2)}

\section{XV-1- Geometry optimization parameters}
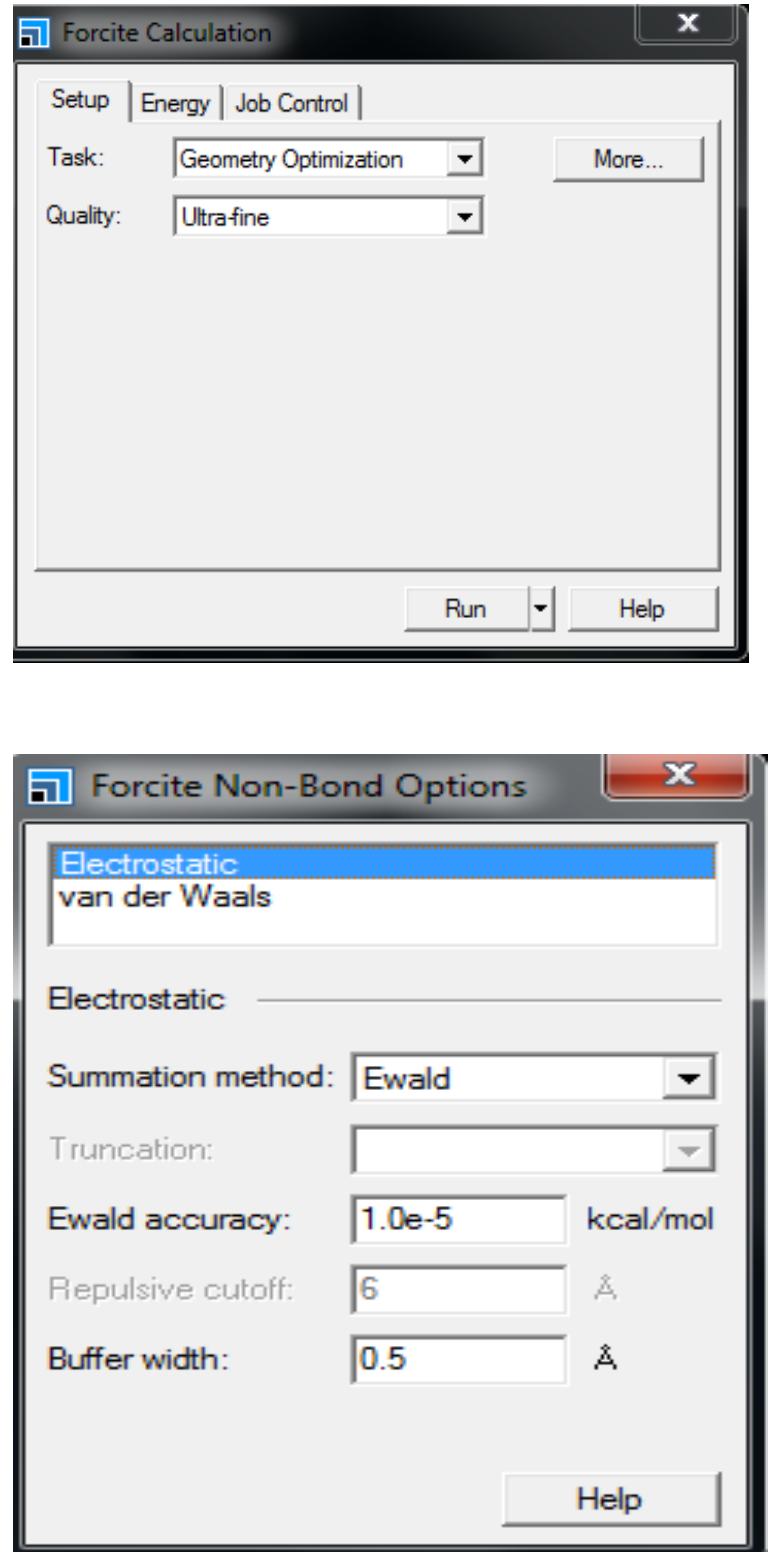

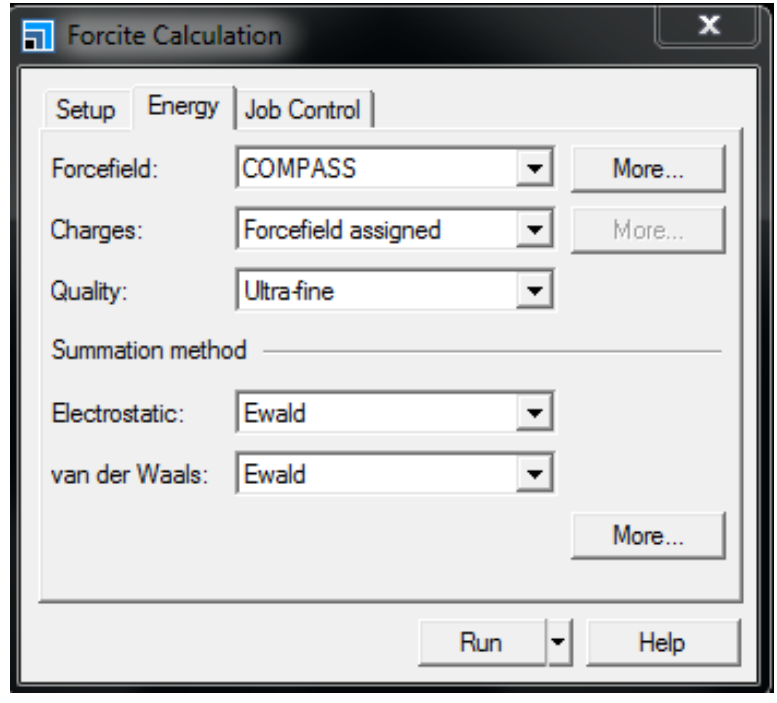

\section{目 Forcite Non-Bond Options}

\section{Electrostatic}

van der Waals

van der Waals

Summation method:

Truncation

Ewald accuracy: $\quad 1.0 \mathrm{e}-5 \mathrm{kcal} / \mathrm{mol}$

Repulsive cutoff: $\quad \sqrt{1}$

Buffer width

0.5 
XV-2- Dynamic simulation parameters
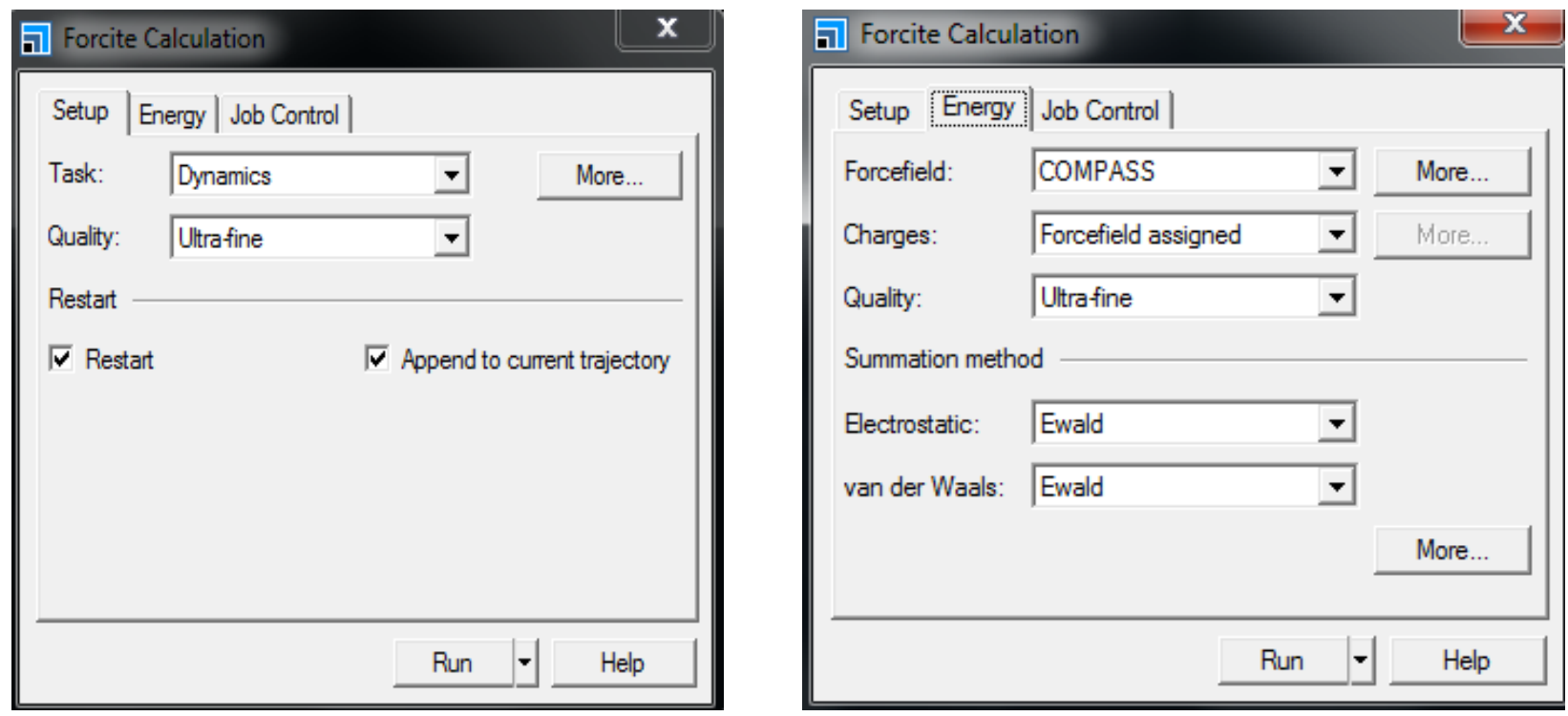

\begin{tabular}{|c|c|c|c|}
\hline 国 Forcite Dynamics & & & \\
\hline Dynamics | Thermosta & at | Barost & at & Advanced \\
\hline Ensemble: & NPT & $\nabla$ & \\
\hline Initial velocities: & Cumrent & $\nabla$ & \\
\hline Temperature: & 298.0 & & K \\
\hline Pressure: & 1.0e-4 & & $\mathrm{GPa}$ \\
\hline Time step: & 0.5 & & fs \\
\hline Total simulation time: & 200.0 & & ps \\
\hline Number of steps: & 400000 & $\div$ & \\
\hline \multirow[t]{2}{*}{ Frame output every: } & 5000 & $\div$ & steps \\
\hline & & & Help \\
\hline
\end{tabular}

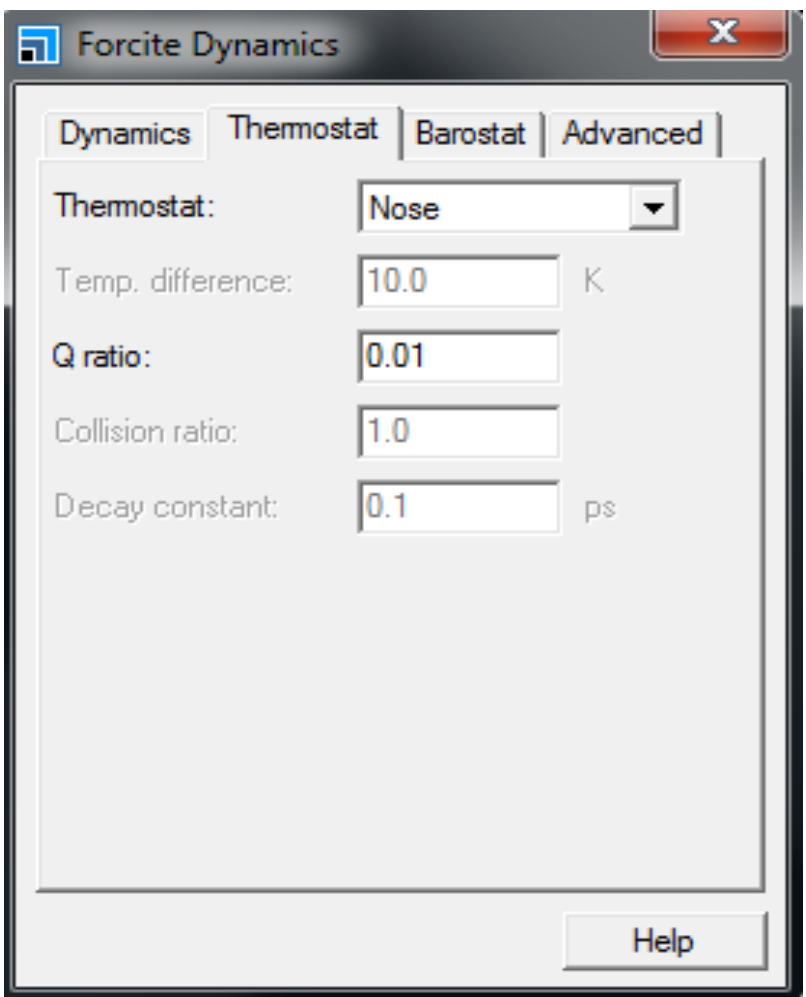



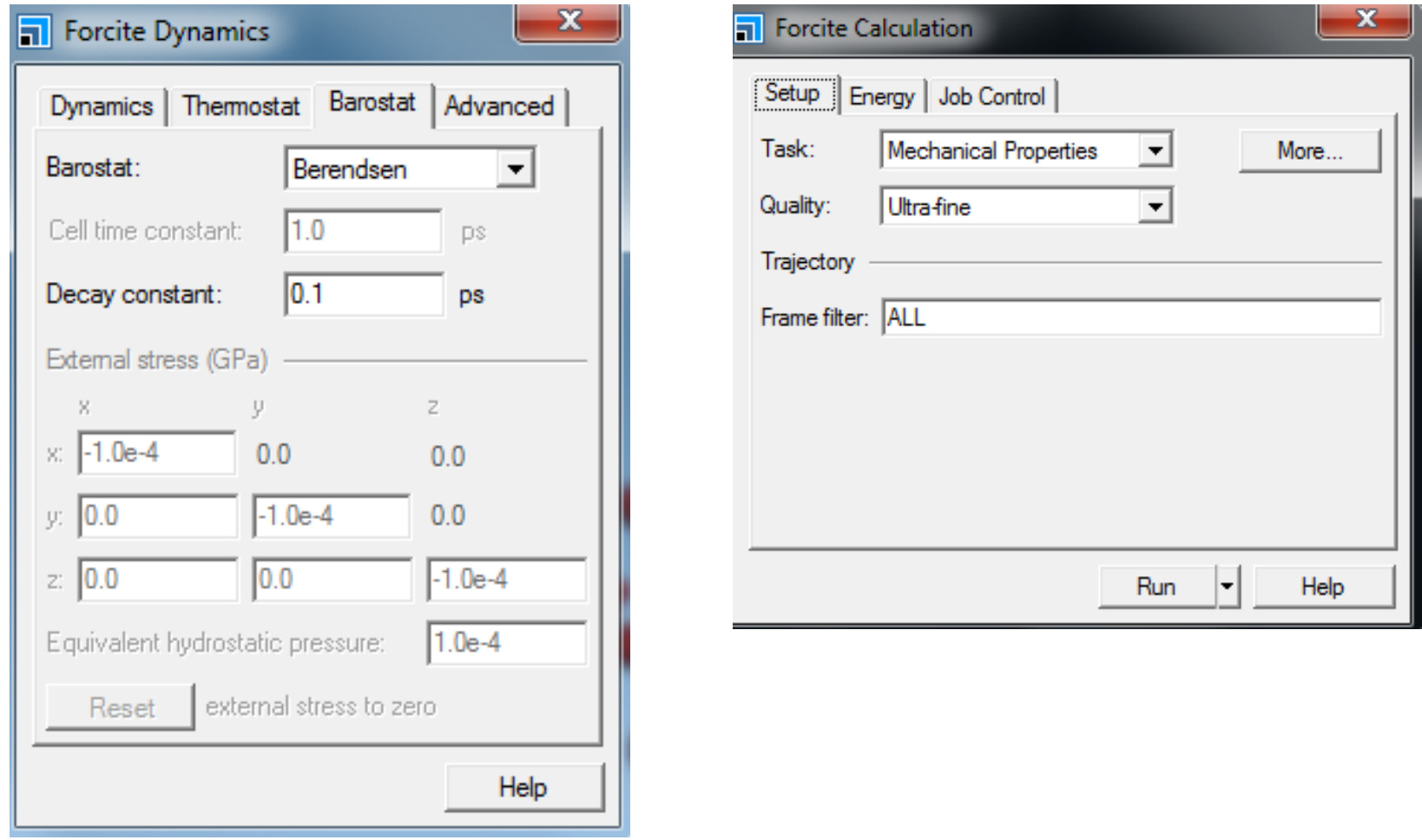

\section{XV-3- Results of J2}

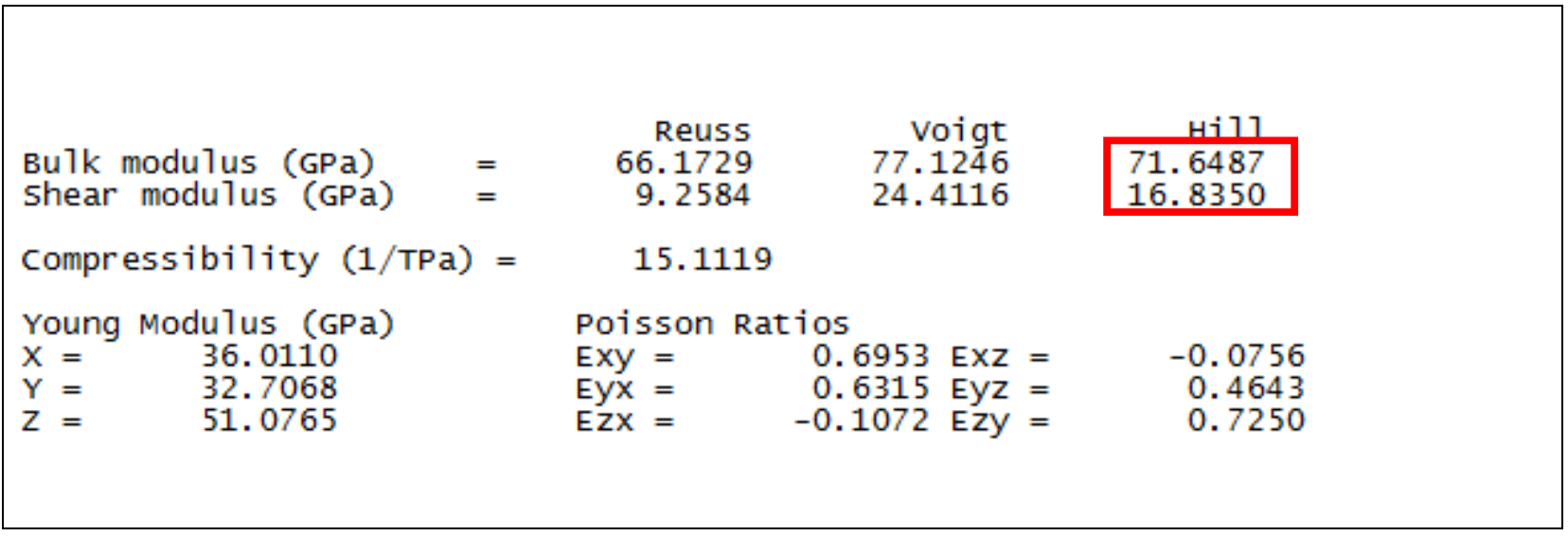




\section{References}

Aierken, Y., Sahin, H., Iyikanat, F., Horzum, S., Suslu, A., Chen, B., . . Peeters, F. M. (2015). Portlandite crystal: Bulk, bilayer, and monolayer structures. Phys. Rev. B Physical Review B, 91(24). doi:10.1103/physrevb.91.245413

Allen, M. P., \& Tildesley, D. (1987). Computer simulation of liquids. Oxford: Clarendon Press.

Askeland, D. R., Phule, P. P., \& Wright, W. J. (2011). The Science and engineering of materials: sixth edition. Mason, OH: Cengage Learning.

Atkins, P. (1978). Physical chemistry. San Francisco: W.H. Freeman.

Barnes, P. (1983). Structure and performance of cements. London: Applied Science.

Bass, J. D. (1995). Elasticity of minerals, glasses, and melts. AGU Reference Shelf Mineral Physics \& Crystallography: A Handbook of Physical Constants, 45-63. doi:10.1029/rf002p0045

Beaud, M. (2006). Comment préparer et rédiger un mémoire de master, une thèse de doctorat ou tout autre travail universitaire à l'ère du Net. Grands repères.

Beaudoin, J., Raki, L., \& Alizadeh, R. (2009). A 29Si MAS NMR study of modified C-S-H nanostructures. Cement and Concrete Composites, 585-590. doi:10.1016/j.cemconcomp.2008.11.004

Becker, O. M., Mackerell, A. D., \& Roux, B. (2001). Computational biochemistry and biophysics. New York: M. Dekker.

Bonaccorsi, E., Merlino, S., \& Kampf, A. (2005). The Crystal Structure of Tobermorite 14 A (Plombierite), a C-S-H Phase. Journal of the American Ceramic Society J American Ceramic Society, 88(3), 505-512. doi:10.1111/j.1551-2916.2005.00116.x

Bonaccorsi, E., Merlino, S., \& Taylor, H. (2004). The crystal structure of jennite, Ca9Si6O18(OH)6.8H2O. Cement and Concrete Research, 34(9), 1481-1488. doi:10.1016/j.cemconres.2003.12.033 
Cement Statistics and Information. (n.d.). Retrieved from USGS: http://minerals.usgs.gov/minerals/pubs/commodity/cement/

Chen, J. J., Thomas, J. J., Taylor, H. F., \& Jennings, H. M. (2004). Solubility and structure of calcium silicate hydrate. Cement and Concrete Research, 34(9), 1499-1519. doi:10.1016/j.cemconres.2004.04.034

Constantinides, G. (2013). Nanoscience and nanoengineering of cement-based materials. Nanotechnology in Eco-Efficient Construction. doi:10.1533/9780857098832.1.7

Constantinides, G., \& Ulm, F.-J. (2007). The nanogranular nature of C-S-H. Journal of the Mechanics and Physics of Solids, 64-90. doi:10.1016/j.jmps.2006.06.003

Detwiler, R. J., Monteiro, P. J., Wenk, H.-R., \& Zhong, Z. (1988). Texture of calcium hydroxide near the cement paste-aggregate interface. Cement and Concrete Research, 18(5), 823-829. doi:10.1016/0008-8846(88)90109-3

Diamond, S. (2004). The microstructure of cement paste and concrete-a visual primer. Cement and Concrete Composites, 26(8), 919-933. doi:10.1016/j.cemconcomp.2004.02.028

Dolado, J. S., Griebel, M., \& Hamaekers, J. (2007). A Molecular Dynamic Study of Cementitious Calcium Silicate Hydrate (C?S?H) Gels. Journal of the American Ceramic Society J American Ceramic Society. doi:10.1111/j.1551-2916.2007.01984.x

Dove, M. T. (n,d). An introduction to atomistic simulation methods - UPV/EHU. Retrieved from http://www.ehu.eus/sem/seminario_pdf/SEM_SEM_4_7-37.pdf

Feldman, R. F., \& Sereda, P. J. (1968). A model for hydrated Portland cement paste as deduced from sorption-length change and mechanical properties. Matériaux et Constructions, 509-520. doi:10.1007/bf02473639

Frenkel, D., \& Smit, B. (2002). Understanding molecular simulation: from algorithms to applications. San Diego: Academic Press. 
Gmira, A. (2003). Etude texturale et thermodynamique d'hydrates modèles du ciment (Unpublished master's thesis). Thèse doctorat: Physico-chimie des matériaux: Orléans.

Hamid, S. A. (1981). The crystal structure of the 11 A natural tobermorite Ca2.25[Si3O7.5(OH)1.5]·1H2O. Zeitschrift für Kristallographie - Crystalline Materials, 154(1-4). doi:10.1524/zkri.1981.154.14.189

Hartman, M., \& Berliner, R. (2006). Investigation of the structure of Ettringite by time-of-flight neutron powder diffraction techniques. Cement and Concrete Research, 36(2), 364-370. doi:10.1016/j.cemconres.2005.08.004

Henderson, D. M., \& Gutowsky, H. S. (1962). A NUCLEAR MAGNETIC RESONANCE DETERMINATION OF THE HYDROGEN POSITIONS IN Ca(OH)2. THE AMERICAN MTNERALOGIST, 47.

Hou, D. (2014). Molecular simulation on the calcium silicate hydrate (C-S-H) gel(Unpublished master's thesis). Hong Kong University of Science and Technology.

Jennings, H. M. (1986). Aqueous Solubility Relationships for Two Types of Calcium Silicate Hydrate. Journal of the American Ceramic Society J American Ceramic Society, 69(8), 614618. doi:10.1111/j.1151-2916.1986.tb04818.x

Jennings, H. M. (2000). A model for the microstructure of calcium silicate hydrate in cement paste. Cement and Concrete Research, 30(1). doi:10.1016/s0008-8846(99)00209-4

Jennings, H. M., Thomas, J. J., Gevrenov, J. S., Constantinides, G., \& Ulm, F.-J. (2007). A multitechnique investigation of the nanoporosity of cement paste. Cement and Concrete Research, 329-336. doi:10.1016/j.cemconres.2006.03.021

Jorge, S., Dolado, a., \& Klaas van, B. (2011). Recent advances in modeling for cementitious materials. Cement and Concrete Reseach, 41, 711-726.

Jupe, A. C., Cockcroft, A. K., Barnes, P., Colston, S. L., Sankar, G., \& Hall, C. (2001). The site occupancy of $\mathrm{Mg}$ in the brownmillerite structure and its effect on hydration properties: an X- 
ray/neutron diffraction and EXAFS study. J Appl Cryst Journal of Applied Crystallography J Appl Crystallogr, 55-61. doi:10.1107/s0021889800016095

Kusuma, G. H., Budidarmawan, J., \& Susilowati, A. (2015). Impact of Concrete Quality on Sustainability. Procedia Engineering, 125(754-759). doi:10.1016/j.proeng.2015.11.122

Lagerblad, B., Jennings, H., \& Chen, J. (1999). Modification of Cement Paste with Silica Fume-A NMR Study. Nanotechnology in Construction Special Publication, 123-131. doi:10.1039/9781847551528-00123

Lea, F. M., \& Hewlett, P. C. (1998). Lea's chemistry of cement and concrete. London: Arnold.

Leach, A. R. (2001). Molecular modelling: principles and applications. Harlow, England: Prentice Hall.

Manzano, H., Dolado, J. S., Guerrero, A., \& Ayuela, A. (2007). Mechanical properties of crystalline calcium-silicate-hydrates: comparison with cementitious $\mathrm{C}-\mathrm{S}-\mathrm{H}$ gels. phys. stat. sol. (a) physica status solidi (a), 204(6), 1775-1780. doi:10.1002/pssa.200675359

Manzano, H., Dolado, J., \& Ayuela, A. (2008). Prediction of the mechanical properties of the major consitituents phases of cementitious systems by atomic simulations. RILEM Proceedings PRO, 58, 47-55.

Manzano, H., Dolado, J., \& Ayuela, A. (2009). Elastic properties of the main species present in Portland cement pastes. Acta Materialia, 57(5), 1666-1674. doi:10.1016/j.actamat.2008.12.007

Mehta, P. K., \& Monteiro, P. J. (2014). Concrete: microstructure, properties, and materials. New York: McGraw-Hill.

Merlino, S., Bonaccorsi, E., \& Armbruster, T. (1999). Tobermorites: Their real structure and orderdisorder (OD) character. American Mineralogist, 84(10), 1613-1621. doi:10.2138/am-19991015 
Merlino, S., Bonaccorsi, E., \& Armbruster, T. (2001). The real structure of tobermorite 11 Å: normal and anomalous forms, OD character and polytypic modifications. European Journal of Mineralogy eur.j.mineral., 13(3), 577-590. doi:10.1127/0935-1221/2001/0013-0577

Merlino, S., Bonaccorsi, E., \& Armbruster, T. (2001). The real structure of tobermorite 11Å: normal and anomalous forms, OD character and polytypic modifications. European Journal of Mineralogy eur.j.mineral., 13(3), 577-590. doi:10.1127/0935-1221/2001/0013-0577

Midgley, C. M. (1952). The crystal structure of $\beta$ dicalcium silicate. Acta Crystallogr Acta Crystallographica Acta Cryst, 5(3). doi:10.1107/s0365110x52000964

Mindess, S., \& Young, J. F. (1981). Concrete. Englewood Cliffs, NJ: Prentice-Hall.

Mondal, P. (2008). Nanomechanical properties of cementitious materials (Unpublished master's thesis).

Mondal, P., \& Jeffery, J. W. (1975). The crystal structure of tricalcium aluminate, Ca3Al2O6. Acta Crystallogr Sect B Acta Crystallogr B Acta Crystallogr B Struct Crystallogr Cryst Chem Acta Crystallogr Sect B Struct Crystallogr Cryst Chem Acta Cryst Sect B Acta Cryst B Acta Crystallographica Section B Structural Crystallography and Crystal Chemis, 31(3), 689-697. doi:10.1107/s0567740875003639

Moon, J. (2013). Experimental and Theoretical Studies on Mechanical Properties of Complex Oxides in Concrete (Unpublished master's thesis).

Moon, J., Yoon, S., \& Monteiro, P. J. (2015). Mechanical properties of jennite: A theoretical and experimental study. Cement and Concrete Research, 71, 106-114. doi:10.1016/j.cemconres.2015.02.005

Odler, I. (2000). Special inorganic cements. London: E \& FN Spon.

Oh, J. E., Clark, S. M., Wenk, H.-R., \& Monteiro, P. J. (2012). Experimental determination of bulk modulus of $14 \AA$ tobermorite using high pressure synchrotron X-ray diffraction. Cement and Concrete Research, 42(2), 397-403. doi:10.1016/j.cemconres.2011.11.004 
Pedersen, B. F., \& Semmingsen, D. (1982). Neutron diffraction refinement of the structure of gypsum, CaSO4.2H2O. Acta Crystallogr Sect B Acta Crystallogr B Acta Crystallogr B Struct Crystallogr Cryst Chem Acta Crystallogr Sect B Struct Crystallogr Cryst Chem Acta Cryst Sect B Acta Cryst B Acta Crystallographica Section B Structural Crystallography and Crystal Chemis, 38(4), 1074-1077. doi:10.1107/s0567740882004993

Pelisser, F., Gleize, P. J., \& Mikowski, A. (2012). Effect of the Ca/Si Molar Ratio on the Micro/nanomechanical Properties of Synthetic C-S-H Measured by Nanoindentation. J. Phys. Chem. C The Journal of Physical Chemistry C, 116(32), 17219-17227. doi:10.1021/jp302240c

Pellenq, R. J.-M., Kushima, A., Shahsavari, R., Vliet, K. J., Buehler, M. J., Yip, S., \& Ulm, F.-J. (2009). A realistic molecular model of cement hydrates. Proceedings of the National Academy of Sciences, 106(38), 16102-16107. doi:10.1073/pnas.0902180106

Popovics, S. (1998). Strength and related properties of concrete: a quantitative approach. New York: Wiley.

Powers, T. (1960). Properties of cement Portland concrete. Proceedings of the Fourth International Symposium of the Chemistry of Cement, 2(1960), 571-609.

Ramachandran, K. I., Deepa, G., \& Namboori, K. (2008). Computational chemistry and molecular modeling: Principles and applications. Berlin: Springer.

Rejmak, P., Dolado, J. S., Stott, M. J., \& Ayuela, A. (2012). 29 Si NMR in Cement: A Theoretical Study on Calcium Silicate Hydrates. J. Phys. Chem. C The Journal of Physical Chemistry C, 116(17), 9755-9761. doi:10.1021/jp302218j

Richardson, I. (1999). The nature of C-S-H in hardened cements. 29(8), 1131-1147. doi:10.1016/s0008-8846(99)00168-4

Richardson, I. (2004). Tobermorite/jennite- and tobermorite/calcium hydroxide-based models for the structure of C-S-H: applicability to hardened pastes of tricalcium silicate, $\beta$-dicalcium silicate, 
Portland cement, and blends of Portland cement with blast-furnace slag, metakaol. 34(9), 1733-1777. doi:10.1016/j.cemconres.2004.05.034

Richardson, I. (2008). The calcium silicate hydrates. Cement and Concrete Research, 38(2), 137-158. doi:10.1016/j.cemconres.2007.11.005

Rieu, J.-F. (2012). Rédigez efficacement vos rapports et thèses. ENI.

Sanchez, F., \& Sobolev, K. (2010). Nanotechnology in concrete - A review. Construction and Building Materials, 2060-2071.

Shahsavari, R., Buehler, M., Pellenq, R. J.-M., \& Ulm, F.-J. (2009). First-Principles Study of Elastic Constants and Interlayer Interactions of Complex Hydrated Oxides: Case Study of Tobermorite and Jennite. Journal of the American Ceramic Society, 92(10), 2323-2330. doi:10.1111/j.1551-2916.2009.03199.x

Speziale, S., Jiang, F., Mao, Z., Monteiro, P. J., Wenk, H.-R., Duffy, T. S., \& Schilling, F. R. (2008). Single-crystal elastic constants of natural Ettringite. Cement and Concrete Research, 38(7), 885-889. doi:10.1016/j.cemconres.2008.02.004

Speziale, S., Reichmann, H., Schilling, F., Wenk, H., \& Monteiro, P. (2008). Determination of the elastic constants of Portlandite by Brillouin spectroscopy. Cement and Concrete Research, 38(10), 1148-1153. doi:10.1016/j.cemconres.2008.05.006

Taylor, H. F. (1956). Relationships Between Calcium Silicates and Clay Minerals. Clay Minerals, 3(16), 98-111. doi:10.1180/claymin.1956.003.16.06

Taylor, H. F. (1986). Proposed Structure for Calcium Silicate Hydrate Gel. Journal of the American Ceramic Society J American Ceramic Society, 69(6), 464-467. doi:10.1111/j.11512916.1986.tb07446.x

Taylor, H. F. (1997). Cement chemistry. London: T. Telford.

Thomas, J. J., Jennings, H. M., \& Allen, A. J. (2010). Relationships between Composition and Density of Tobermorite, Jennite, and Nanoscale $\mathrm{CaO}-\mathrm{SiO} 2-\mathrm{H} 2$ O. J. Phys. Chem. C The Journal of Physical Chemistry C, 114(17), 7594-7601. doi:10.1021/jp910733x 
Torre, Á. G., Vera, R. N., Cuberos, A. J., \& Aranda, M. A. (2008). Crystal structure of low magnesium-content alite: Application to Rietveld quantitative phase analysis. Cement and Concrete Research, 38(11), 1261-1269. doi:10.1016/j.cemconres.2008.06.005

Tunega, D., \& Zaoui, A. (2010). Understanding of bonding and mechanical characteristics of cementitious mineral tobermorite from first principles. J. Comput. Chem. Journal of Computational Chemistry, 32(2), 306-314. doi:10.1002/jcc.21622

Vandamme, M., Ulm, F.-J., \& Fonollosa, P. (2010). Nanogranular packing of C-S-H at substochiometric conditions. Cement and Concrete Research, 40(1), 14-26. doi:10.1016/j.cemconres.2009.09.017

Velez, K., Maximilien, S., Damidot, D., Fantozzi, G., \& Sorrentino, F. (2001). Determination by nanoindentation of elastic modulus and hardness of pure constituents of Portland cement clinker. Cement and Concrete Research, 31(4), 555-561. doi:10.1016/s0008-8846(00)005056

Wu, Z.-Q., \& Young, F. J. (1984). FORMATION OF CALCIUM HYDROXIDE FROM AQUEOUS SUSPENSIONS OF TRICALCIUM SILICATE. Chemischer Informationsdienst, 15-19. doi:10.1002/chin.198419015

Yoshimura, H. N., Molisani, A. L., Narita, N. E., Cesar, P. F., \& Goldenstein, H. (2007). Porosity dependence of elastic constants in aluminum nitride ceramics. Mat. Res. Materials Research, 10(2), 127-133. doi:10.1590/s1516-14392007000200006 